\title{
Global Analysis of Protein Phosphorylation Regulation upon Stimulation of Exocytosis in the Nerve Terminal
}

\author{
Dissertation \\ for the award of the degree \\ "Doctor rerum naturalium" \\ of the Georg-August-Universität Göttingen
}

within the doctoral program Molecular Biology

of the Georg-August University School of Sciences (GAUSS)

submitted by

Mahdokht Kohansal Nodehi

from

Tehran, Iran

Göttingen, 2016 
Thesis committee members:

Supervisor, reviewer:

\section{Prof. Dr. Reinhard Jahn}

Department of Neurobiology

Max-Planck Institute for Biophysical Chemistry

Göttingen, Germany

PhD committee member, reviewer:

Prof. Dr. Henning Urlaub

Bioanalytical Mass Spectrometry Group

Max-Planck Institute for Biophysical Chemistry Göttingen, Germany

PhD committee member:

\section{Prof. Dr. Jürgen Wienands}

Institute for Cellular and Molecular Immunology

University of Medical Center Göttingen

Göttingen, Germany

Date of the oral examination: November 24th, 2016 
I hereby declare that I prepared this PhD thesis, entitled "Global Analysis of Protein Phosphorylation Regulation upon Stimulation of Exocytosis in the Nerve Terminal", on my own and with no other sources and aids than those cited.

Mahdokht Kohansal Nodehi

October 2016, Göttingen 
تقديم به بلر و مادرم 


\section{Table of Contents}

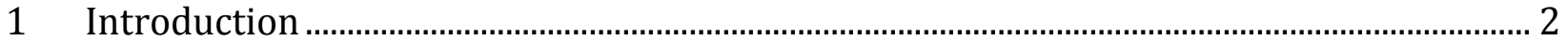

1.1 Synaptic vesicle recycling ........................................................................................ 2

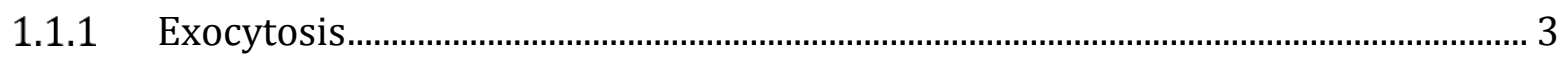

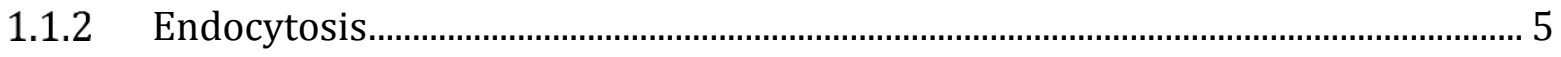

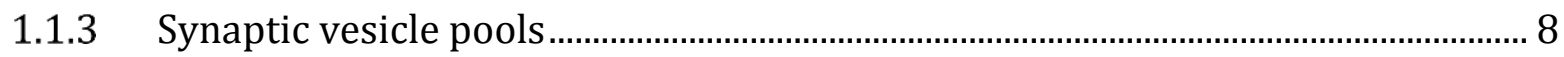

1.2 Synaptosomes as a model to study exocytosis/endocytosis........................................... 9

1.3 Activity-dependent regulation of synaptic vesicle recycling .........................................11

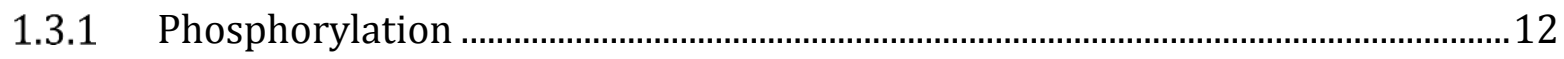

1.3.2 Modulation of synaptic vesicle recycling by phosphorylation ..............................13

1.3.2.1 Exocytosis related phosphoproteins....................................................................14

1.3.2.2 Endocytosis related phosphoproteins................................................................16

1.3.2.3 Cytoskeleton related phosphoproteins..............................................................16

1.3.3 Kinases and phosphatases of the nerve terminal ...................................................17

1.3.3.1 Calcium/calmodulin-dependent protein kinase II (CaMKII)..........................18

1.3.3.2 Protein kinase A (PKA).................................................................................2

1.3.3.3 Protein kinase C (PKC) ..................................................................................20

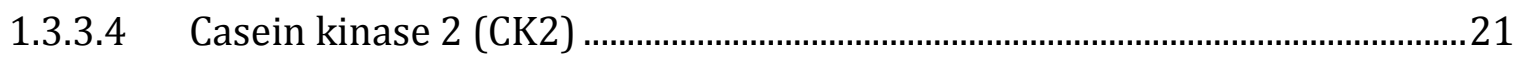

1.3.3.5 Extracellular-signal Regulated Kinase 1/2 (ERK1/2) …………………….....22

1.3.3.6 Cyclin-dependent kinase 5 (CdK5) ................................................................22

1.3.3.7 Glycogen synthase kinase 3 (GSK-3) ............................................................23

1.3.3.8 Protein phosphatase 1 (PP1) ........................................................................2

1.3.3.9 Protein phosphatase 2A (PP2A) ………………………………………......24

1.3.3.10 Protein phosphatase 2B (PP2B, Calcineurin) ……………………………......24

1.3.4 Modulation of synaptic vesicle pools via kinases/phosphatases ...........................24

1.4 Mass spectrometry (MS)-based phosphoproteomics ………………………………....25

1.4.1 Protein Identification.....................................................................................25

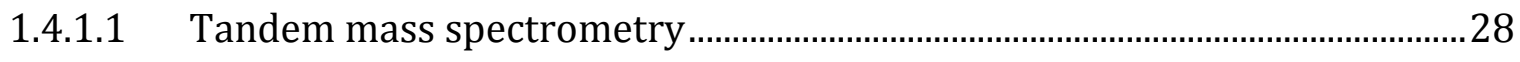

1.4.1.2 Sample ionization .......................................................................................2

1.4.1.3 Peptide sequencing .......................................................................................29 


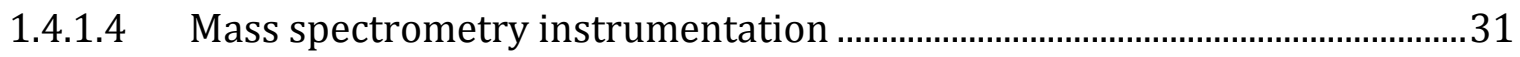

1.4.1.5 Peptide and protein identification ........................................................................... 32

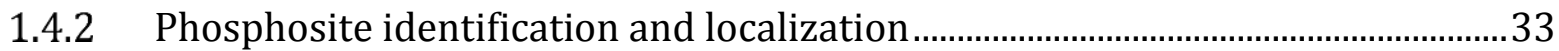

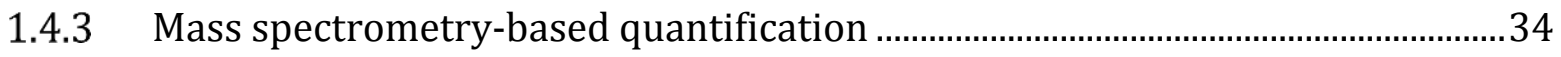

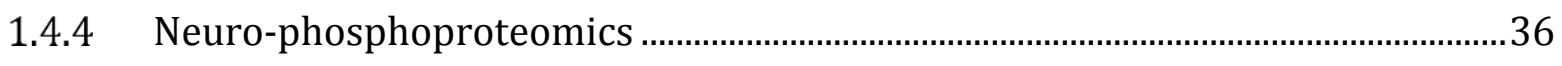

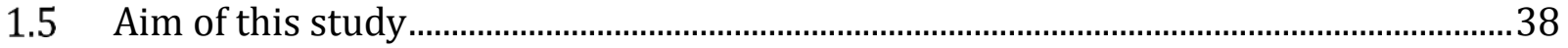

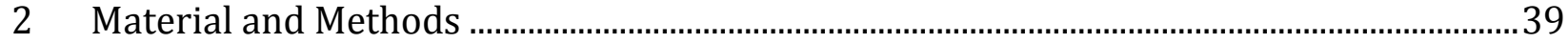

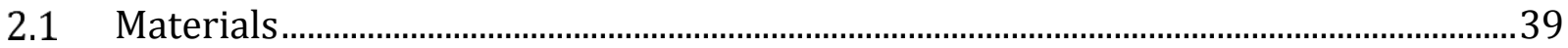

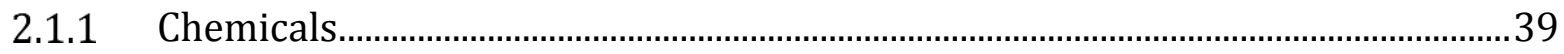

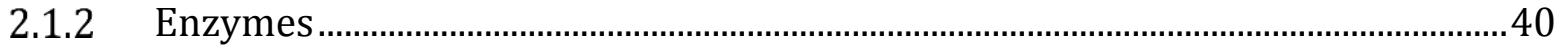

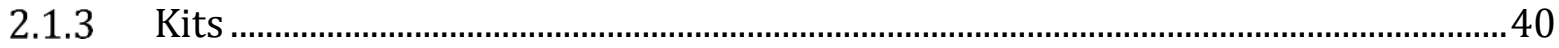

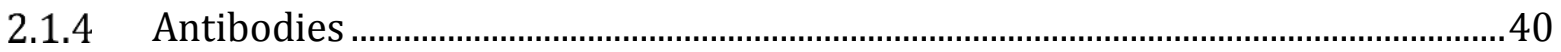

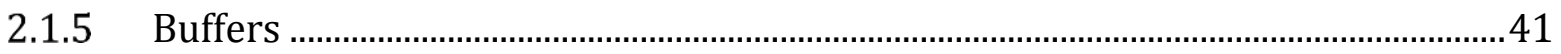

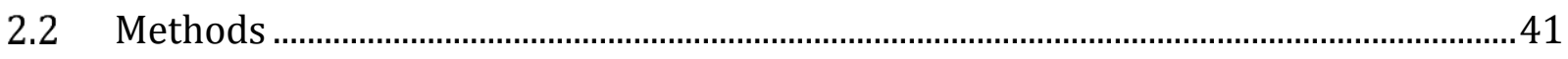

2.2.1 Biochemical Methods................................................................................................ 41

2.2.1.1 Synaptosomes preparation.................................................................................. 41

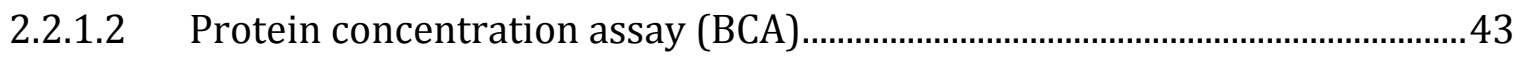

2.2.1.3 Synaptosomes stimulation (Glutamate Release Assay)...................................43

2.2.1.4 Sample collection for mass spectrometry......................................................... 44

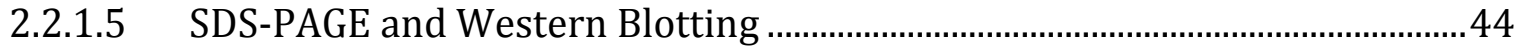

2.2.1.6 In vitro kinase assays for mapping of the kinases responsible for the

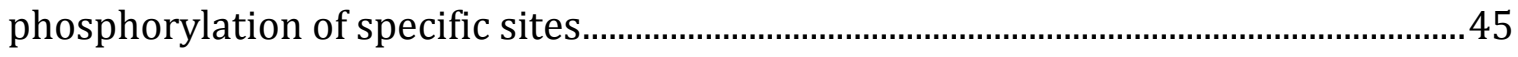

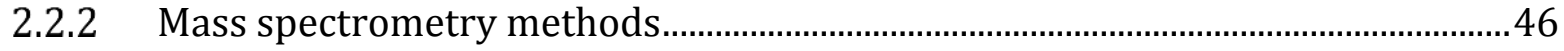

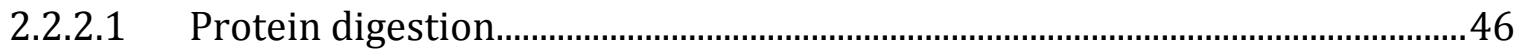

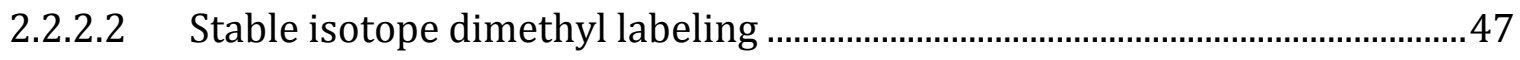

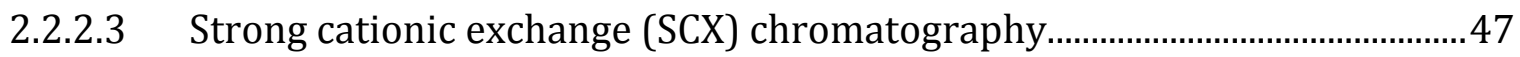

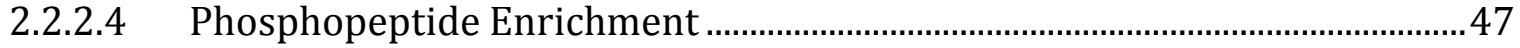

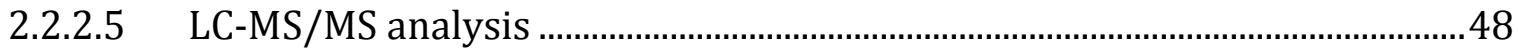

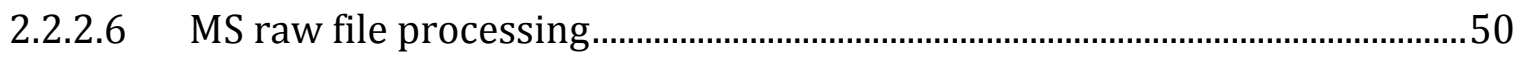

2.2.2.7 Data processing and bioinformatics analysis ..................................................... 51

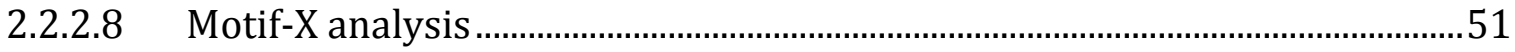

2.2.2.9 Other tools, software and databases ......................................................................52

3 Results 
3.1 Establishment of a protocol for the identification and quantification of

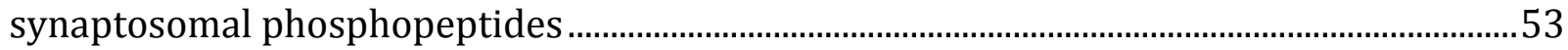

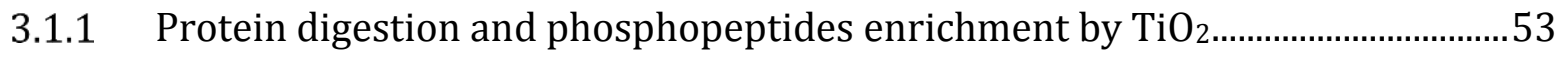

3.1.2 Impact of fractionation on the number of identified phosphopeptides................54

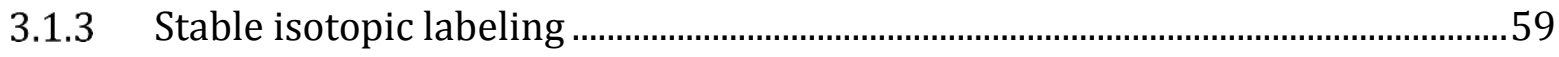

3.1.4 Impact of dimethyl labeling on the number of quantified phosphosites.............60

3.2 Phosphoproteomics of isolated nerve terminals under different stimulation

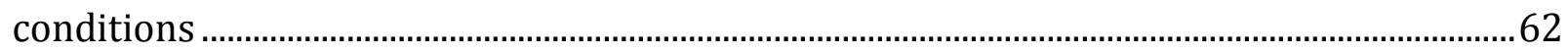

3.2.1 Functional analysis of synaptosomes...................................................................63

3.2.2 Activity-dependent phosphorylation changes of presynaptic proteins................63

3.2.3 Functional/localization grouping of regulated phosphosites..................................70

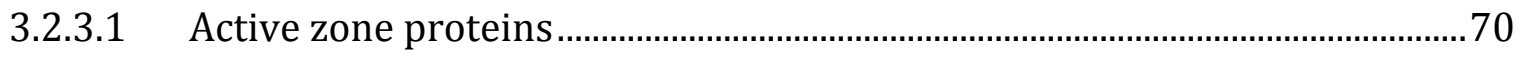

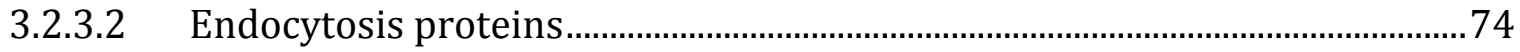

3.2.3.3 Synaptic vesicle and exocytosis related proteins.............................................76

3.2.3.4 Cytoskeleton and associated proteins ................................................................78

3.2.3.5 Ion Channels and transporters....................................................................79

3.2.4 Mapping Kinases and phosphatases responsible for the phosphorylation changes upon stimulation..................................................................................................

3.3 Identification of the presynaptic substrates of CaMKII and Calcineurin (PP2B)......84

3.3.1 Impact of Calcineurin inhibition on the exocytosis and phosphorylation status of the presynaptic proteins

3.3.2 Impact of CaMKII inhibition on the exocytosis and phosphorylation status of the presynaptic proteins

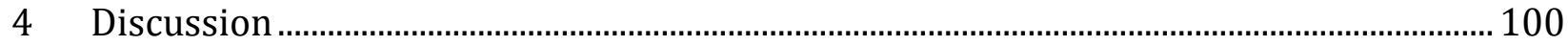

4.1 Activity-dependent phosphorylation changes of nerve terminal proteins .............. 100

4.1.1 $\mathrm{Ca}^{2+}$ ions are the main regulator of phosphorylation upon stimulation...........100

4.1.2 Impact of active zone proteins phosphorylation on synaptic transmission ... 101

4.1.3 Phosphorylation of cytoskeleton-associated proteins regulates exo- and endocytosis........................................................................................................................ 102

4.1.4 Multisite phosphorylated proteins ……………………………………………... 103

4.2 Possible role of Calcineurin in RRP pool replenishment and kinase regulation .... 104

4.3 CaMKII might function as the major regulator of kinases/phosphatases upon

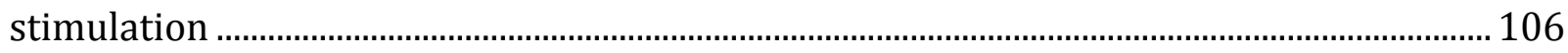


4.4 Activity of kinases and phosphatases is tightly coordinated in the nerve terminal 108

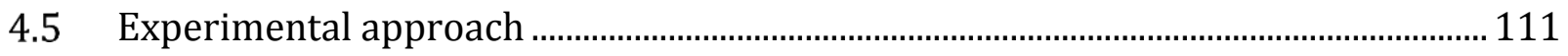

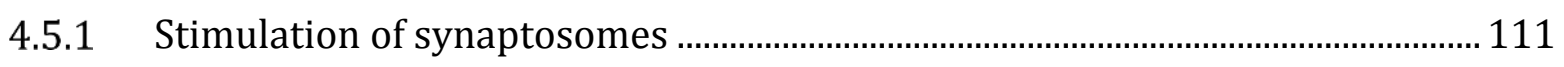

4.5.2 Time-course of stimulation-dependent phosphorylation changes..................... 113

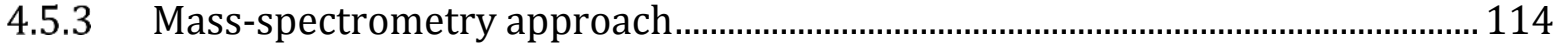

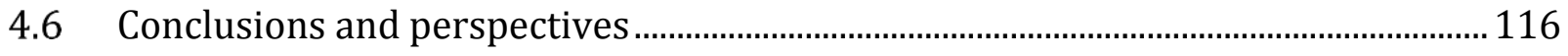

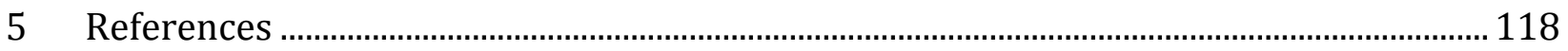

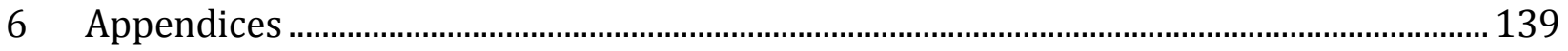

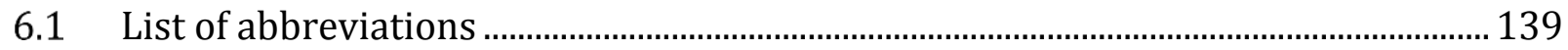

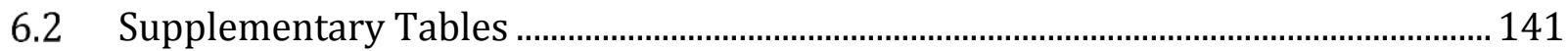

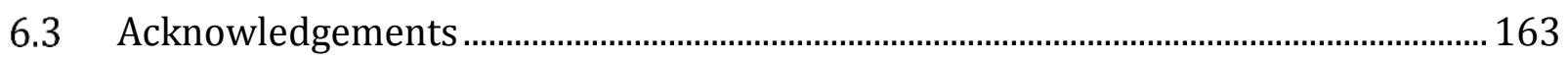




\section{List of Figures}

Figure 1-1: The key steps of synaptic vesicle recycling.

Figure 1-2: Protein components clathrin-mediated endocytosis are recruited sequentially. 6

Figure 1-3: Suggested modes of synaptic vesicle recycling.

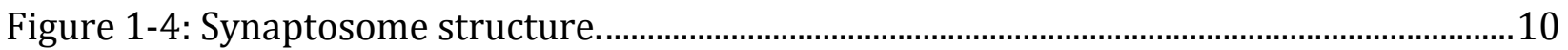

Figure 1-5: Phosphorylation of amino acids investigated in this work.......................................13

Figure 1-6: The protein kinase reaction........................................................................................ 18

Figure 1-7: Functional domains in the primary structure of calcium/calmodulin-dependent

protein kinase II (CaMKII).

Figure 1-8: Functional domains in the primary structure of protein kinase C (PKC) isoforms

Figure 1-9: The general workflow of a shotgun (phospho)proteomics .......................................26

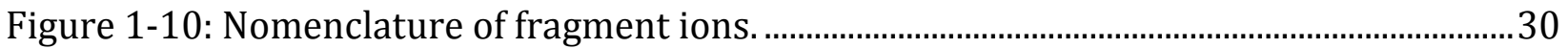

Figure 1-11: The schematic view of a Q-Exactive HF instrument. ................................................ 31

Figure 1-12: Labeling scheme of the reactions occurs in stable isotope dimethyl labeling...35

Figure 2-1: The flow diagram of purification of synaptosome from rat brains. ........................42

Figure 2-2: Conversion of glutamate to $\alpha$-ketoglutarate is coupled to the reduction of NADP+

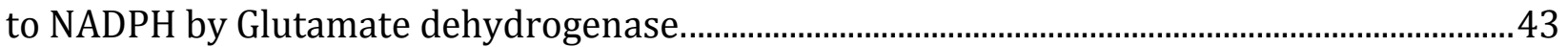

Figure 3-1: Optimization of protein digestion and $\mathrm{TiO}_{2}$ phosphopeptides enrichment.........55

Figure 3-2: Effect of strong cation exchange chromatography on the identification of

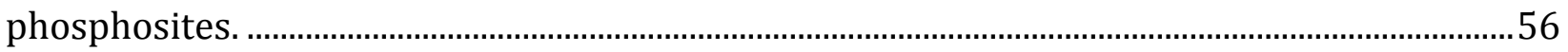

Figure 3-3: Effect of starting material on the identification of phosphopeptides....................57

Figure 3-4: Comparison between $\mathrm{TiO}_{2}$ and IMAC phosphopeptides enrichment methods. ...58

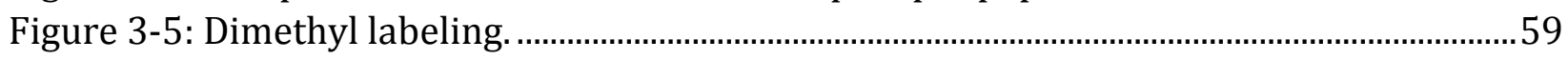

Figure 3-6: Effect of dimethyl labeling on the number of quantified phosphosites.................61

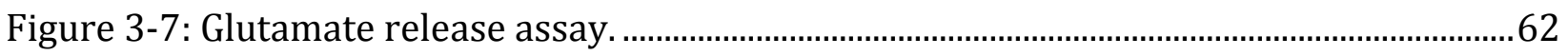

Figure 3-8: Functional assessment of synaptosomes...................................................................... 64

Figure 3-9: Experimental workflow for quantitative phosphoproteomics of synaptosomes in

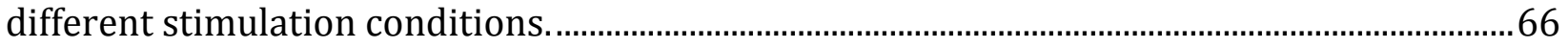

Figure 3-10: Overview of the quantified phosphosites in the three comparisons.....................68

Figure 3-11: Overlap of quantified phosphosites in different comparisons. .............................69

Figure 3-12: Functional/localization categorization of the regulated phosphosites in the three comparisons. ..................................................................................................................... 71

Figure 3-13: Quantified phosphosites of active zone in the $\mathrm{K}^{+}, \mathrm{Ca}^{2+}$ versus $\mathrm{K}^{+}$, EGTA

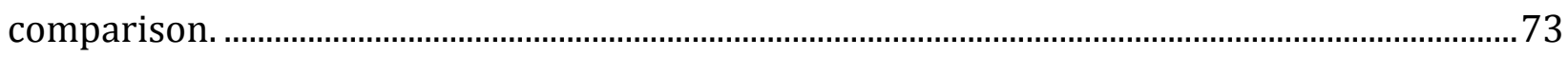

Figure 3-14: Phosphosites related to clathrin-mediated endocytosis..........................................75

Figure 3-15: Regulation of synapsin 1 phosphosites upon stimulation.......................................77

Figure 3-16: Motif analysis of the regulated phosphosites and experimental verification of

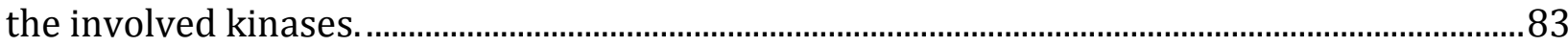

Figure 3-17: Experimental workflow for quantitative phosphoproteomics of synaptosomes

treated with Calcineurin and CaMKII inhibitors. 85 
Figure 3-18: Glutamate release assay and immunoblot analysis of synaptosomes treated with Cyclosporine A.

Figure 3-19: Overview of the quantified phosphosites of synaptosomes treated with Cyclosporine A.

Figure 3-20: Motif analysis of the putative substrates of Calcineurin.

Figure 3-21: Motif analysis of the downregulated phosphosites by Calcineurin inhibition.. 92

Figure 3-22: Glutamate release assay and immunoblot analysis of synaptosomes treated with $\mathrm{KN}-93$.

Figure 3-23: Overview of the quantified phosphosites of synaptosomes treated with KN-93.

Figure 3-24: Analysis of the downregulated phosphosites by CaMKII inhibition.

Figure 4-1: CaMKII inhibition downregulates PKA and PKC activity indirectly through the

downregulation of PDK-1.

Figure 4-2: Activity of kinases and phosphatases is tightly coordinated in the nerve terminal upon stimulation.

Figure 4-3: $\mathrm{KCl}$ stimulation versus electrical stimulation.

Figure 4-4: Dynamics of glutamate release and protein phosphorylation by $\mathrm{KCl}$ versus

electrical stimulation.

\section{List of Tables}

Table 1-1: Categorization of the most commonly used quantitative approaches of proteomics. 34

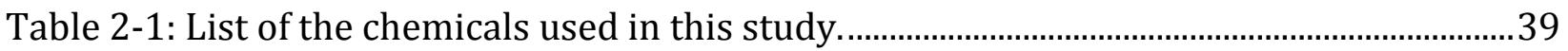

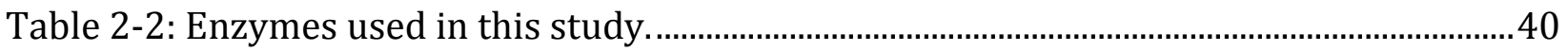

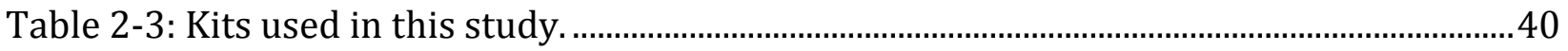

Table 2-4: Antibodies used in this study, WB (Western Blot). ........................................................40

Table 2-5: List of buffers and their composition used in this study.............................................41

Table 2-6: Enzymes, control peptides and chemicals used in in vitro kinase assays. .............45

Table 2-7: Sequence of peptides used in in vitro kinase assay.....................................................46

Table 6-1: List of regulated phosphosites in comparison $\mathrm{K}^{+}$, EGTA vs $\mathrm{K}^{+}$, $\mathrm{Ca}^{2+} \ldots \ldots \ldots \ldots . . . . . . . . . . .141$

Table 6-2: List of regulated phosphosites in comparison EGTA vs $\mathrm{K}^{+}, \mathrm{Ca}^{2+}$........................... 145

Table 6-3: List of regulated phosphosites in comparison EGTA vs $\mathrm{K}^{+}$, EGTA ......................... 150

Table 6-4: List of upregulated phosphosites upon Calcineurin inhibition (putative

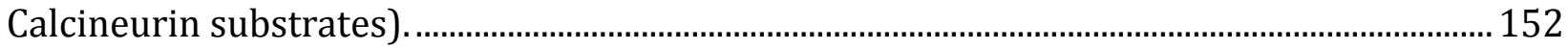

Table 6-5: List of downregulated phosphosites upon Calcineurin inhibition. ....................... 154

Table 6-6: List of downregulated phosphosites upon CaMKII inhibition.................................. 156 


\section{Abstract}

In chemical synapses, neurons communicate with each other via two mechanisms known as fast and slow synaptic transmission. Fast synaptic transmission happens within milliseconds and is triggered by the arrival of an action potential to the nerve terminal, resulting in the release of neurotransmitters. In contrast, the slow synaptic transmission occurs over hundreds of millisecond to minutes and involves elevation of second messengers such as $\mathrm{Ca}^{2+}$ ions and cAMP. Second messengers directly or indirectly modulate activity of kinases and protein phosphatases (PP) and consequently regulate properties of their substrates via phosphorylation/dephosphorylation.

Up to date, the regulation of synaptic transmission by phosphorylation of presynaptic proteins has been studied by targeted and low-throughput approaches. In the current study, we have applied tandem mass spectrometry-based phosphoproteomics workflow to globally investigate the molecular mechanism of slow synaptic transmission through dynamic changes of the nerve terminal protein phosphorylation. Comparison of the stimulated nerve terminals phosphoproteome in the presence and the absence of extracellular $\mathrm{Ca}^{2+}$ ions revealed that $\mathrm{Ca}^{2+}$ plays a major role in the regulation of protein phosphorylation, while membrane depolarization has a minor role. Moreover, it was demonstrated that components of active zone protein group undergo extensive phosphorylation changes during stimulation. Noteworthy many phosphosites of large scaffolding proteins bassoon and piccolo were dephosphorylated.

In the next step, considering the role of $\mathrm{Ca}^{2+}$ in the regulation of phosphosites, the pharmacological specific inhibitors of a $\mathrm{Ca}^{2+}$-dependent kinase (CaMKII) and a phosphatase (Calcineurin) were used to investigate their role in regulation of the phosphosites. Calcineurin inhibition revealed that many of regulated active zone phosphosites are Calcineurin substrates. In addition, it was shown that, phosphorylation of critical phosphosites were downregulated upon CaMKII inhibition.

Overall, our data suggest that upon the stimulation of nerve terminal activity of CaMKII, PKC, PKA, ERK1/2, CK2, PP2A, and Calcineurin is upregulated, whereas activity of CdK5, GSK-3, PP1 is downregulated. In conclusion, we propose a model in which $\mathrm{Ca}^{2+}$ has a primary role in regulation of tightly interconnected network of kinases and phosphatases upon stimulation. 


\section{Introduction}

\subsection{Synaptic vesicle recycling}

Neuronal communication at the chemical synapses is mediated by release of chemical signals (neurotransmitters) from the presynaptic neurons into a space that separates the pre and postsynaptic neurons called the synaptic cleft. Neurotransmitters are stored in the spherical, membrane-bounded organelle called synaptic vesicle (SV) that consists of proteins and phospholipids (Takamori et al., 2006). Upon arrival of an action potential to the nerve terminal, it leads the depolarization of the membrane and opening of the voltage-gated calcium channels. This leads to the influx of calcium ions into the nerve terminal. It has been estimated that SV release is detectable when the concentration of the calcium ions in the nerve terminal reaches 1-2 $\mu \mathrm{M}$ and is saturated by more than $20 \mu \mathrm{M}$ (Bollmann et al., 2000). The increase of calcium ion concentration results in the fusion of the SVs to the presynaptic membrane (exocytosis) and release of neurotransmitter into the synaptic cleft. Then neurotransmitters diffuse toward the postsynapse and bind to their respective receptors that are located on the membrane of the postsynaptic neuron. There are two types of postsynaptic receptors. First, ionotropic receptors containing a membrane-spanning domain that function as the ion channel and the second, metabotropic receptors that do not have the ion channel as a part of their structure. However, upon binding of the neurotransmitter, intermediate molecules called G-proteins are activated and interact with the ion channels and regulate them. The opening of the postsynaptic ion channels directly by ionotropic receptors or indirectly by metabotropic receptors results in changes in ion permeability of the postsynapse membrane and, consequently it changes the membrane potential and triggers the sequence of the subsequent events in the postsynaptic neurons. Meanwhile, in the presynaptic part, the membranes of the fused SVs are retrieved from the plasma membrane (endocytosis) and actively refilled by neurotransmitters to be ready for the next round of exocytosis. The combination of exo- and endocytosis together with the neurotransmitter filling of the SVs is called synaptic vesicle recycling (Figure 1-1). Neurotransmitter release by chemical synapses requires SV recycling to refill the SV pool, clear the release site and prevent continuous expansion of the plasma membrane. In the following sections, a brief overview of the two main events of SV recycling pathway (exo- and endocytosis) as well as different pools of the SVs in the nerve terminal will be discussed. 


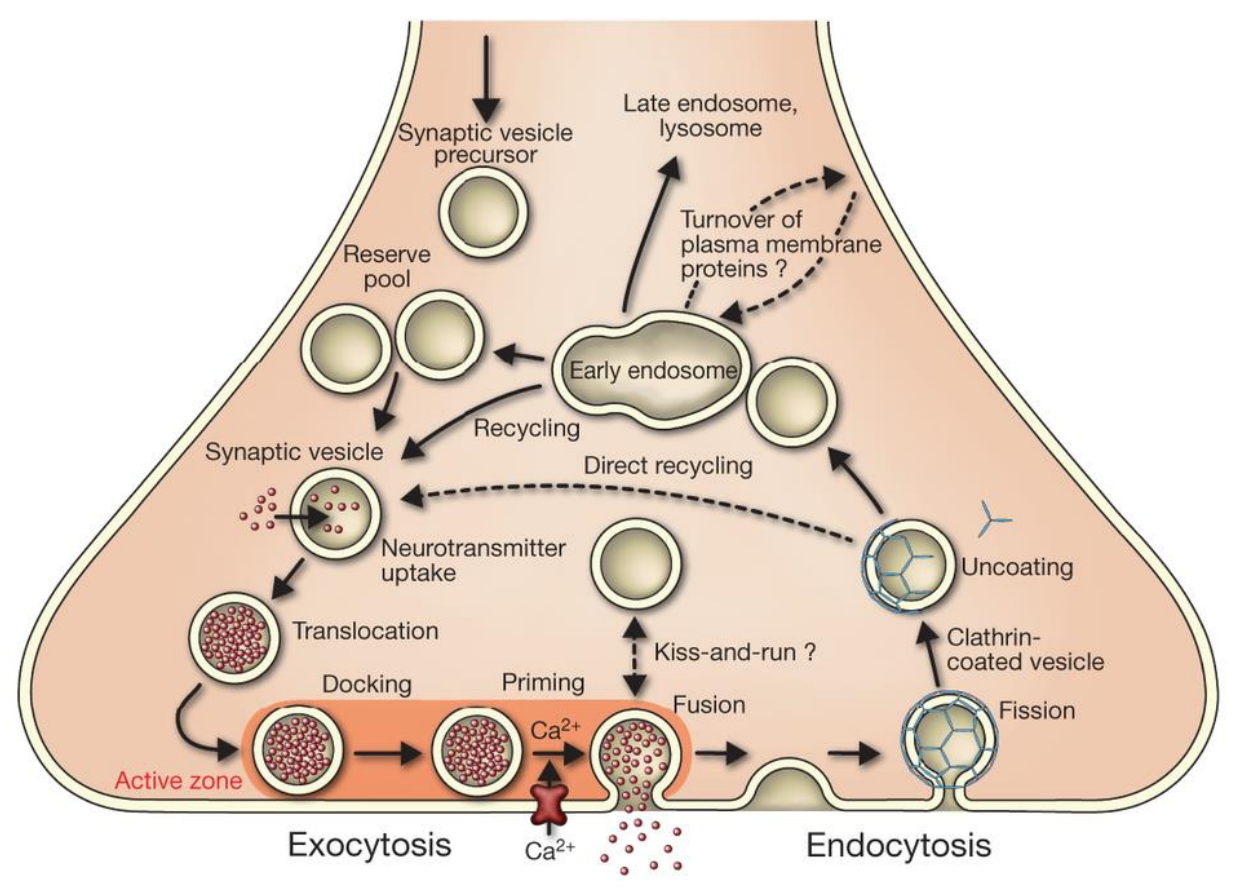

Figure 1-1: The key steps of synaptic vesicle recycling.

In the chemical synapses, neurons communicate with each other via release of neurotransmitters. Filled synaptic vesicles (SVs) with neurotransmitters undergo docking and priming steps in a specialized site of presynaptic membrane called active zone. The arrival of action potential opens voltage-gated calcium channels, resulting in the influx of $\mathrm{Ca}^{2+}$ ions into the nerve terminal. Elevated $\mathrm{Ca}^{2+}$ concentration triggers SVs to fuse to the membrane and release the neurotransmitters. SVs can make a transient fusion pore to release the neurotransmitters recycled by a mechanism called 'Kiss and Run' or completely fuse to the plasma membrane and later be retrieved by clathrin-mediated endocytosis. The newly retrieved clathrin-coated vesicles undergo an uncoating step, during which they lose the clathrin coat. Later, the vesicles are regenerated probably via involvement of early endosomes and refilled with neurotransmitter for the next round of exocytosis. Figure adapted from (Jahn and Fasshauer, 2012).

\subsubsection{Exocytosis}

The fusion of synaptic vesicle into the presynaptic plasma membrane is a complex and highly regulated process that involves different sets of proteins in each step. SVs fusion occurs in the part of the membrane specialized for release called active zone. Primarily, SVs are located in close proximity of the active zone. This step is known to be regulated by two of the active zone proteins, bassoon, and piccolo (Hallermann et al., 2010, Gundelfinger et al., 2015), as well as the actin filaments and motor proteins such as Myosin V (Doussau and Augustine, 2000). In the next step, SVs dock to the active zone via interaction of Rab3a (SV protein) with RIM (the central organizer of the active zone) (Coleman et al., 2007). Followed by docking, SVs interact with several proteins in the active zone such as Munc13 that prepares them for the fast fusion upon arrival of an action potential (priming step). On the molecular level, the 
docking and priming steps share a lot of proteins, and it is difficult to experimentally assign a group of proteins to each of them.

The primed vesicles fuse to the plasma membrane with the assistance of several proteins, for instance, SNARE proteins (soluble N-ethylmaleimide-sensitive factor (NSF) attachment protein receptors) that are crucial for the SV fusion. Synaptobrevin (also known as vesicleassociated membrane protein or VAMP2) is one of the SNARE proteins that is located on the SVs. Two other members of the SNARE protein, Syntaxin and SNAP-25 are localized on the plasma membrane. The three proteins form an $\alpha$-helical trans-SNARE complex that functions as the engine for membrane fusion (Jahn and Fasshauer, 2012). SNARE proteins are enough for the SV fusion in vitro. However, SV fusion in the physiological context requires other proteins such as Munc18 and Munc13. Deletion of Munc18 or Munc13 and their respective orthologues completely blocks the release of neurotransmitters (Verhage et al., 2000, Varoqueaux et al., 2002). Munc18 is known to bind to syntaxin and inhibits the formation of trans-SNARE complex but is also needed for the efficient SNARE nucleation (Jahn and Fasshauer, 2012). Munc13 is known as the priming factor that facilitates the conformational change of syntaxin from close to open and subsequently the formation of the SNARE complex (Ma et al., 2013). As it was mentioned above, fusion is triggered by influx of $\mathrm{Ca}^{2+}$ ions through voltage-gated calcium channels mainly by P/Q-type (CaV2.1) or N-type (CaV2.2), and less likely by R-type (CaV2.3) and l-type (CaV1 series), resulting in high concentrated $\mathrm{Ca}^{2+}$ microdomains close to the plasma membrane that are sensed by two calcium sensor proteins involved in the SV fusion, synaptotagmin 1 and Complexins. Synaptotagmin has two calcium binding domains, $\mathrm{C} 2 \mathrm{~A}$ and $\mathrm{C} 2 \mathrm{~B}$. In the presence of calcium, $\mathrm{C} 2$ domains bind to the acidic phospholipids of the membrane (Chapman, 2008) and to syntaxin alone or in the SNARE complex (Rizo et al., 2006) promoting the SV fusion. Complexins are also known to bind to the SNARE complex and play the dual inhibitory and stimulatory role in fusion. It binds to the surface of the SNARE complex and promotes its zippering (Xue et al., 2010). It also competes with synaptobrevin to interact with C-terminal of the SNARE complex (Kummel et al., 2011). $\mathrm{Ca}^{2+}$ is known to be buffered fast and efficiently from the cytoplasm (Meinrenken et al., 2002) resulting in the termination of the release.

After fusion of the SVs to the plasma membrane, the SNARE complex remains assembled in the plasma membrane. An $\mathrm{AAA}^{+}$ATPase called N-ethylmaleimide-sensitive factor (NSF) 
hydrolyzes ATP to disassociate SNARE complex and recycle SNARE proteins (Ryu et al., 2016). The SVs components together with the SNARE complex are retrieved from the membrane by endocytosis to be used for the next round of exocytosis.

\subsubsection{Endocytosis}

Clathrin-Mediated Endocytosis (CME) is the best-studied pathway for the recycling of SVs (Jung and Haucke, 2007). The main proteins involved in the building of the clathrin coats are clathrin itself and the clathrin adaptor protein called adaptor protein 2 (AP2). Coat assembly is initiated in a region close to the active zone called periactive zone (Haucke et al., 2011) with the recruitment of the AP2 to capture the SV proteins and the clathrin. AP2 connects the clathrin to the membrane. As more adaptor proteins and clathrin are recruited, the coat acquires more curvature (Jung and Haucke, 2007, Dittman and Ryan, 2009). Several other adaptor proteins are involved in formation of the coats. For example, AP180 that is known to bind to synaptobrevin and sort it into the vesicles. AP180 also binds to AP2 as well as phosphoinositides. The latter binding is known to regulate clathrin polymerization (Saheki and De Camilli, 2012). The curvature of the coat is initially achieved by the structure of the clathrin that makes triskelia shape by the assembly of the three light and three heavy chains of clathrin molecules (Jung and Haucke, 2007, Saheki and De Camilli, 2012). The curvature is then stabilized by other adaptor proteins such amphiphysin and endophilin (Itoh and De Camilli, 2006) that contain a domain called Bin, Amphiphysin, Rvs (BAR) domain (McMahon and Gallop, 2005). Epsin and its isoforms (Eps15) are another set of adaptor proteins that induce the curvature in coats via interaction ENTH domain and PtdIns(4,5)P2. This domain is known to bind to clathrin and other components of the endocytosis machinery (Horvath et al., 2007). In the next step, the budded coated vesicles are dissociated via a fission machinery and mainly by a GTPase called dynamin (Saheki and De Camilli, 2012). At the C-terminal, dynamin has a proline-rich domain that binds to the SH3 domain of other endocytotic proteins such as amphiphysin, endophilin, intersectin and syndapin, as well as of synaptojanin (Dittman and Ryan, 2009). The newly formed synaptic vesicles are uncoated by an ATPase called Hsc70 and its cofactor auxilin. It is known that auxilin binds to AP2 and therefore targets the Hsc70 to the coated vesicles (Eisenberg and Greene, 2007). Uncoating 
also involves synaptojanin, which is known to have a role in destabilization of the coat assembly (Dittman and Ryan, 2009) (Figure 1-2).

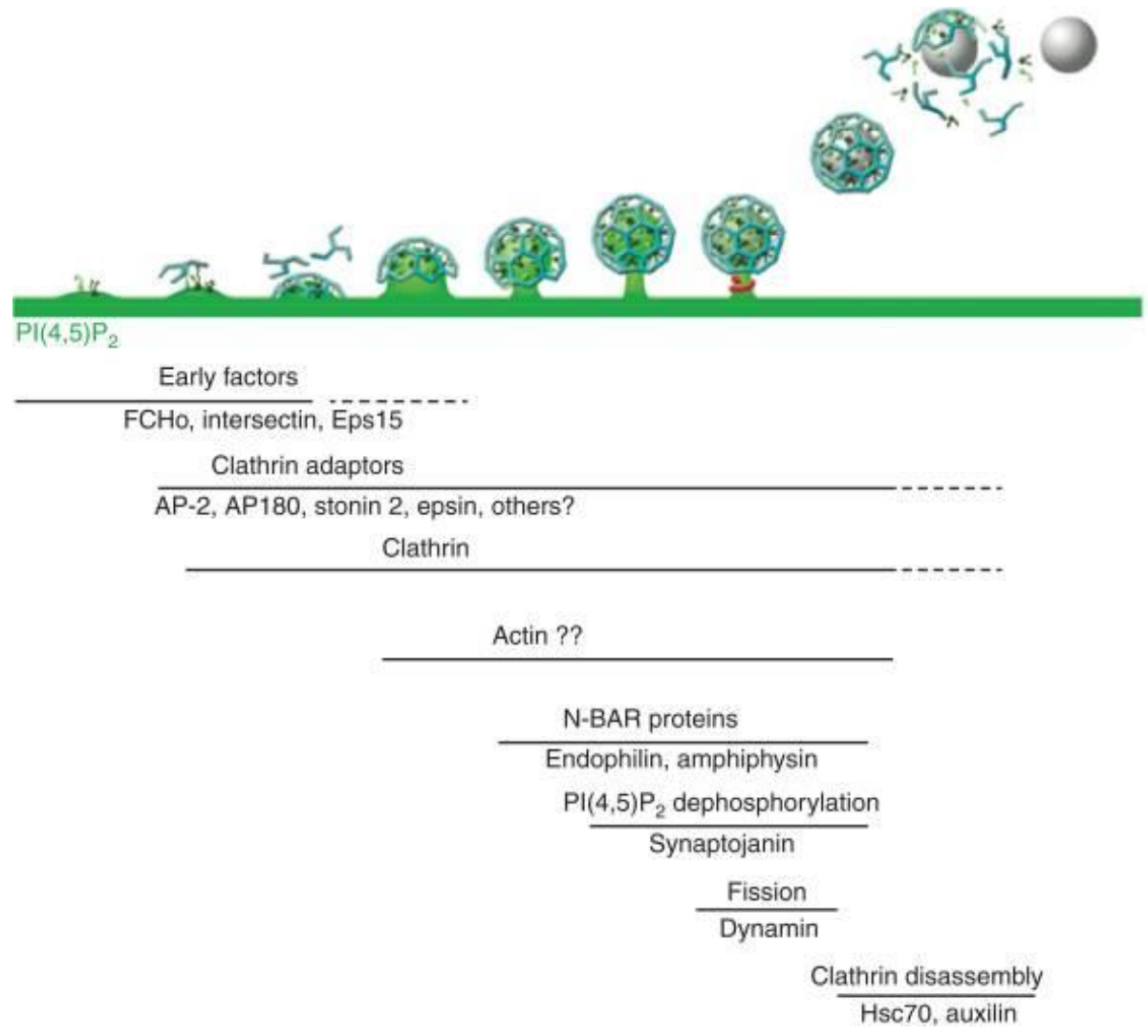

Figure 1-2: Protein components clathrin-mediated endocytosis are recruited sequentially.

Formation of clathrin-coated endocytotic pits during endocytosis requires the sequential recruitment of a variety of proteins in different steps. At the early step PI(4,5)P, AP2 and, intersectin nucleate the pit formation. Following, clathrin, AP180, stonin 2 induce the curvature of the pit. The curvature is stabilized by the action of N-BAR proteins such as Endophilin and amphiphysin. The latter proteins also recruit dynamin that is involved in fission of coated pits from the membrane and synaptojanin, Hsc70 and auxilin that cooperate with each other in the SV uncoating. Figure is adapted from (Saheki and De Camilli, 2012).

Beside the clathrin-mediated endocytosis, three alternative endocytosis pathways were proposed to occur in the synapse (Figure 1-3): Kiss-and-Run, Ultra-fast clathrin-independent endocytosis and Activity-Dependent Bulk Endocytosis (ADBE) (Soykan et al., 2016). In contrast to CME, Kiss-and-Run pathway occurs in the active zone. In this model, synaptic vesicles do not completely fuse to the plasma membrane, but make a transient, nanometer pore between the SV membrane and the plasma membrane to release the neurotransmitter (Rizzoli and Jahn, 2007). Therefore the composition of the SVs remain intact and can be recycled very fast. In fact the Kiss-and-Run pathway $(<1 \mathrm{sec})$ is much faster than CME $(10-20$ 
sec) (Soykan et al., 2016). In addition, high-pressure freezing electron microscopy revealed another even faster pathway called ultrafast endocytosis. Similar to CME, it happens in the periactive zone and is dependent on dynamin (Watanabe et al., 2013, Watanabe et al., 2014). Furthermore, after a long and strong stimulation, neurons undergo ADBE that does not specifically recycle SVs but is rather an emergency response to prevent the expansion of the synaptic plasma membrane (Nguyen et al., 2014, Nicholson-Fish et al., 2015). The parameters that determine which of the mentioned endocytosis pathways occur are the frequency of the stimulation, temperature, the type of the neurons and possibly the distinct pool of the synaptic vesicles. Below the variety of the synaptic vesicle pools will be explained.

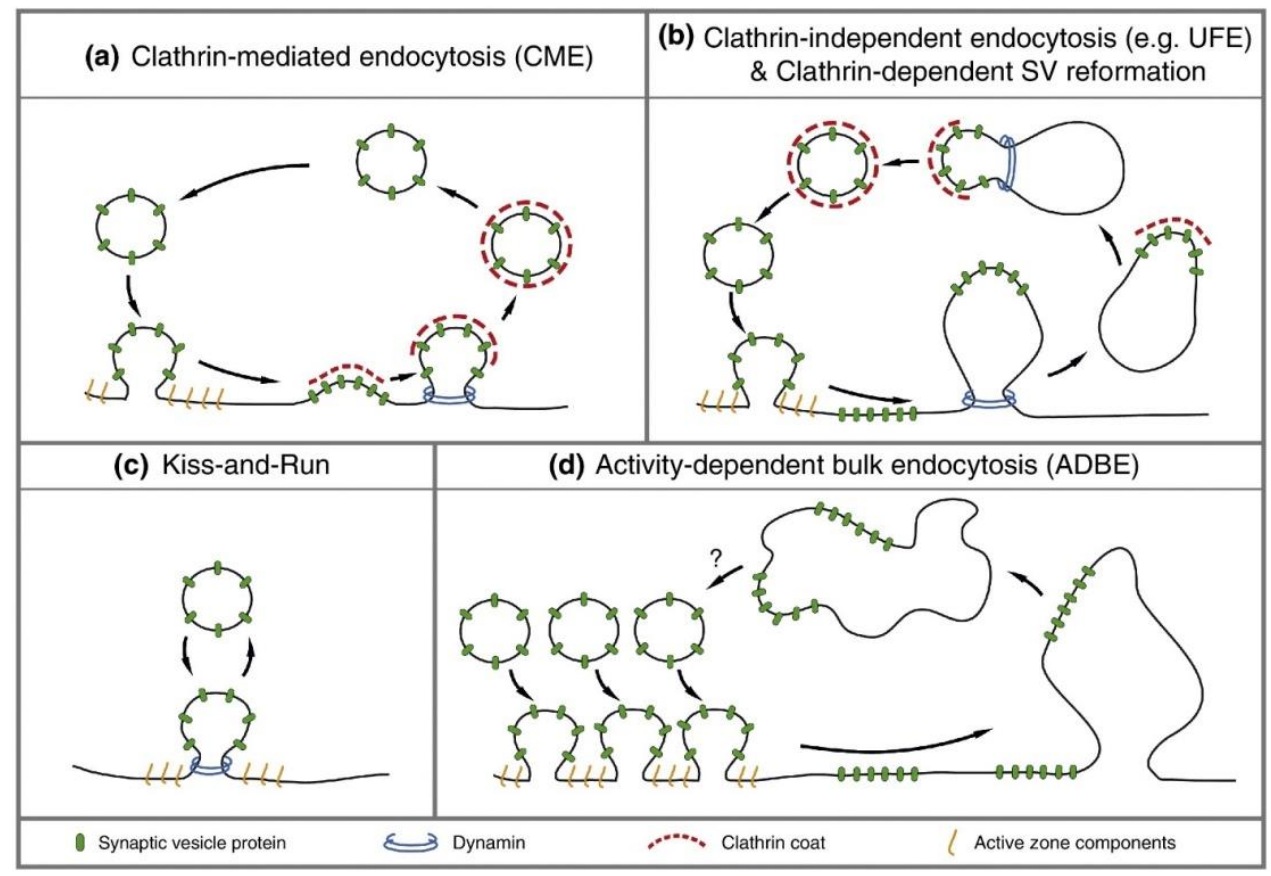

Figure 1-3: Suggested modes of synaptic vesicle recycling.

SVs are fused completely to the membrane and retrieved by the formation of clathrin-coated pits. (b) Following complete fusion of SVs to the membrane, they are retrieved via formation of vacuoles-like structure and later clathrin and AP-2 are recruited to generate SVs from the endosomal-like vacuoles. (c) SVs do not fuse completely to the membrane, rather they make small pores to release the neurotransmitter and are retrieved directly from the active zone. (d) Upon simultaneous fusion of multiple SVs, the membrane is retrieved via formation of the bulk endosomes to retrieve the SVs. The figure is adapted from (Soykan et al., 2016). 


\subsubsection{Synaptic vesicle pools}

One of the main features of the chemical synapses is the ability to store the synaptic vesicles in the nerve terminal. It is estimated that 100-200 vesicles are stored in the nerve terminal of hippocampal neurons. Under the electron microscope these vesicles are similar and do not show significant differences in their properties. However, different studies indicated that distinct populations of SVs (SV pools) exist in the nerve terminal that can be categorized based on their different release capacities. The well-established model that has been proposed and is still valid in the field is the three vesicle pools model containing the readily releasable pool, recycling pool and the reverse pool (Rizzoli and Betz, 2005). Below I will discuss the SV pools in more detail.

Readily releasable pool (RRP) -The RRP is a part of the recycling pools that contains vesicles ready to fuse to the membrane upon stimulation. These vesicles are known to have the highest release probability (Waters and Smith, 2002). RRP in the hippocampal boutons is depleted within $2 \mathrm{sec}$ of $20 \mathrm{~Hz}$ stimulation. The RRP vesicles are docked to the active zone, therefore they are the least mobile pool in the nerve terminal (Ruiz et al., 2011). Interestingly, it has been suggested that within the RRP, not all the docked vesicles have the similar release parameter and some of them are faster than the others (Hanse and Gustafsson, 2001).

Recycling pool - The recycling pool is defined as the pool of vesicles that can undergo recycling repeatedly upon the mild stimulation. It is estimated that it contains $5-20 \%$ of all vesicles. Recycling pool vesicles replenish the fused RRP pool vesicles through the transition of RP to RRP vesicles which is a rate-limiting step during persistent activity of neuron (Alabi and Tsien, 2012). These vessels are not docked to the active zone and during the resting condition have high mobility.

Reverse pool- Vesicles of the reverse pool are reluctant to be released and only upon an intense stimulation (10-100 $\mathrm{Hz}$ for many seconds or minutes) and after RRP and recycling pool fuse to the membrane. The reverse pool contains $80-90 \%$ of all the vesicles. Using the temperature-sensitive Drosophila dynamin mutant shibire, it was shown that this pool is recruited for the release after the recycling pool even in low-frequency stimulation (Hayashi et al., 2008). The reverse pool vesicles are scattered in the nerve terminal and have low mobility (Rizzoli and Betz, 2005). 
It was generally assumed that the difference in the release abilities of the SV pools is due to their localization regarding to the active zone. RRP are docked and ready to fuse, recycling pool is located behind them and ready to be substituted with RRP and reverse pool vesicles are located more distant from the active zone. Although this assumption is correct for the RRP vesicles that are docked to the active zone, evidence shows that recycling pool and the reserve pool intermix with each other and are not recognized spatially (Harata et al., 2001, de Lange et al., 2003). Nevertheless, still the recycling and the reserve pools are distinguishable by their different release kinetics and reverse pool vesicles are more reluctant to be released and also less mobile. Therefore, it has been suggested that there should be a mechanism that specifically keeps the reverse pool vesicles tight to each other and inhibits their release. It has been proposed that synapsin protein interlinks the vesicles to each other and also links vesicles to the actin filaments in the nerve terminal (Gitler et al., 2004). Therefore, the vesicles with the higher frequency of recycling have less synapsin and remain more mobile.

\subsection{Synaptosomes as a model to study exocytosis/endocytosis}

Synaptosomes represent nerve terminals of neurons that were detached from the rest of the axon and cell body during preparation. Their membrane reseals and makes a close environment containing synaptic vesicles and other subcellular organelles (Figure 1-4). Synaptosomes were initially isolated in 1958 by Wittaker (Whittaker, 1993), and later they were characterized by electron microscopy and biochemical assays. It is known that synaptosomes contain all of the molecular machinery that is necessary for the storage and the release of neurotransmitters. Besides vesicles, synaptosomes contain functional mitochondria that are able to produce ATP to keep them active. Synaptosomes are known to be able to hold the cytoplasmic $\mathrm{Ca}^{2+}$ concentration in the range of $100-200 \mathrm{nM}$ in the presence of $2 \mathrm{mM}$ extracellular $\mathrm{Ca}^{2+}$ (Verhage et al., 1988). Furthermore, they are able to maintain the membrane potential via their functional ion channels and transporters such as $\mathrm{Na}^{+} / \mathrm{K}^{+}$ ATPase. Preparation of high-quality synaptosomes that are metabolically stable and responsive to the stimulus is highly dependent on their isolation methodology. The high quality synaptosomes remain active for 3-4 hours after the preparation (Breukel et al., 1997). For any phosphoproteomics study, the desired phosphopeptides have to be enriched (see 
section 1.4.2. for more details). Since synaptosomes are enriched for nerve terminal proteins, they make an ideal model for nerve terminal phosphoproteomics. This increases the probability of nerve terminal phosphopeptide identification compared to other subcellular fractions. All of the mentioned properties make synaptosome an excellent while simple system that has been used extensively to study the regulation of neurotransmission and synaptic vesicle recycling events such as exo- and endocytosis. Several methods have been used to depolarize the membrane of synaptosomes and evoke the neurotransmission release. Below I will discuss the three most common approaches.

A

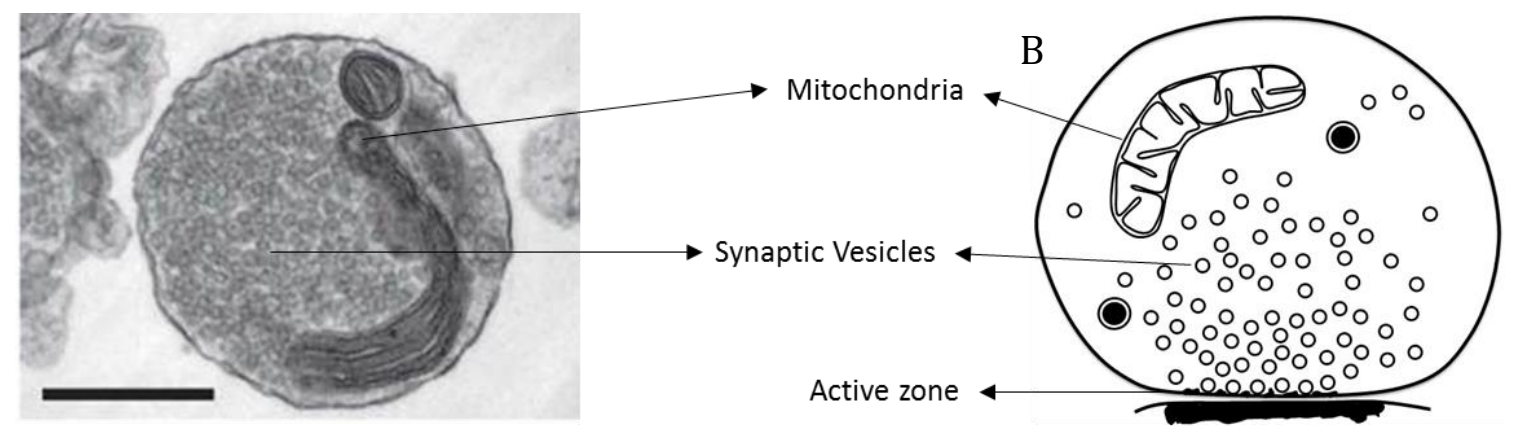

Figure 1-4: Synaptosome structure.

Electron microscopy (A) and schematic image (B) of synaptosomes. Synaptosomes are the detached nerve terminals of neurons from the axon and cell body that contain sub-cellular organelles such as mitochondria and synaptic vesicles as well as functional membrane transporters and ion channels. Synaptosomes have been used widely as a model to study exo- and endocytosis. Scale bars shows $0.5 \mu \mathrm{m}$, adapted from (Daniel et al., 2012)

High concentration of $\boldsymbol{K}^{+}$ions - In this method the potassium gradient is clamped by increasing the extracellular potassium concentration (10-50 $\mathrm{mM}$ ) resulting in the depolarization of the membrane. This opens the voltage-gated calcium channels and increases intracellular calcium ion concentration that initiates the neurotransmitter release (Nicholls and Sihra, 1986). Elevated $\mathrm{KCl}$ introduces a biphasic release of glutamate from the synaptosomes, a rapid phase that is complete within 1-5 sec and a slow phase that is half complete within $52 \mathrm{sec}$. After approximately $2 \mathrm{~min}$ the release reaches a plateau for several minutes. It is known that applying $30 \mathrm{mM} \mathrm{KCl}$ results in a transient spike in the intracellular $\mathrm{Ca}^{2+}$ concentration to $600 \mathrm{nM}$, flowing by a plateau that lasts for several minutes (McMahon and Nicholls, 1991). 
Block of the potassium channels - 4-aminopyridine (1-10 mM) is commonly used to block the potassium channels. In contrast to the clamped depolarization produced by $\mathrm{KCl}$, it probably induces repetitive firing of action potentials since the release is sensitive to Tetrodotoxin (sodium channels inhibitor) (Tibbs et al., 1989). Similar to KCl stimulation, 4aminopyridine initiates biphasic release of glutamate. Upon application of 4-aminopyridine, the $\mathrm{Ca}^{2+}$ concentration in the nerve terminal is increased up to $500 \mathrm{nM}$ followed by a plateau (in contrast to $\mathrm{KCl}$ where no spike is seen). Overall, it has been suggested that 4-aminopyridine evoked depolarization is closer to in vivo condition because it generates repetitive firing of action potential that is sensitive to Tetrodotoxin (McMahon and Nicholls, 1991).

Electrical stimulation - Synaptosomes are also responsive to electrical pulses. It has been shown that synaptosomes are able to release the amino acids (Bradford, 1970) in a $\mathrm{Ca}^{2+}$ dependent manner upon electrical stimulation without disturbance of their structures (Rodina et al., 1980). However, most of the studies with electrical stimulation of synaptosomes have applied long lasting (20-30 $\mathrm{min}$ ) pulses to investigate the metabolic responses of synaptosomes. There is very limited knowledge available regarding the neurotransmission release upon electrical stimulation (Kuo and Dodd, 2011).

\subsection{Activity-dependent regulation of synaptic vesicle recycling}

It is well established that elevation of $\mathrm{Ca}^{2+}$ ions in the nerve terminal upon stimulation regulates the synchronous and also asynchronous neurotransmitter release. The short burst of synchronous release of neurotransmitters is mostly explained by the appearance of elevated $\mathrm{Ca}^{2+}$ at nano/microdomains in the vicinity of voltage-dependent calcium channels (Neher and Sakaba, 2008). However, the asynchronous release is a result of the extended elevation of cytosolic $\mathrm{Ca}^{2+}$ concentration upon repetitive stimulation of the nerve terminals. Stimulation of nerve terminals not only opens the voltage-gated calcium channels, it also triggers the release of calcium from the intracellular sources (Nizami et al., 2010). Two different types of molecular $\mathrm{Ca}^{2+}$ sensors in the nerve terminal are able to sense the elevation $\mathrm{Ca}^{2+}$ concentration: low-affinity $\mathrm{Ca}^{2+}$ sensors such as synaptotagmins that are able to sense the high concentration of $\mathrm{Ca}^{2+}$ in the micro-domains and are responsible for the synchronous neurotransmitter release and the high-affinity $\mathrm{Ca}^{2+}$ sensors such as CaM (Chin and Means, 2000) that have several effectors, including the Munc13, Myosin V and Rab3a, which are 
involved in the synchronous and asynchronous release (Igarashi and Watanabe, 2007). Elevated $\mathrm{Ca}^{2+}$ also modulates the activity of the kinases and phosphatases present in the nerve terminal, resulting in regulation of the phosphorylation status of the proteins. Below I will discuss the main characterization of protein phosphorylation as the most abundant post translational modification (PTM) and the role of phosphorylation in neurotransmission and SV recycling.

\subsubsection{Phosphorylation}

It is known that the reversible phosphorylation of proteins modulates their activity, proteinprotein interactions and their subcellular localization (Hunter, 2007). Phosphorylation occurs mostly on the serine $(\mathrm{S})$, threonine $(\mathrm{T})$ and tyrosine $(\mathrm{Y})$ amino acids (Figure 1-5). Serine was found to be the most frequent phosphorylated amino acid, followed by threonine and tyrosine (based on phosphorylation database, phosphositeplus.org). It is known that tyrosine phosphorylation is structurally somewhat different from two other counterparts. The phosphate group on tyrosine is located further from the peptide backbone than on serine and threonine, providing more binding specificity (Hunter, 2014). Tyrosine phosphorylation can be detected by the specific phosphotyrosine-domain of proteins such as Src homology 2 (SH2) and phosphotyrosine-binding (PTB) domain (Manning et al., 2002). In addition, the phosphoserine and phosphothreonine residues are recognized by the five binding modules, including 14-3-3 proteins, WW domains, forkhead-associated (FHA) domains and WD40 repeats/LRR modules in F-box proteins. These domains are present in the proteins that are involved in various cellular processes such as ubiquitination, cell cycle or DNA repair (Yaffe and Elia, 2001). In addition to $S, T$, and $Y$, phosphorylation is reported to occur on basic amino acids such as lysine, arginine, and histidine. The phosphorylation of basic amino acids was initially observed in prokaryotes. However, recently they have been reported in the eukaryotes and their role in the signaling pathways has has been studied (Ciesla et al., 2011). 


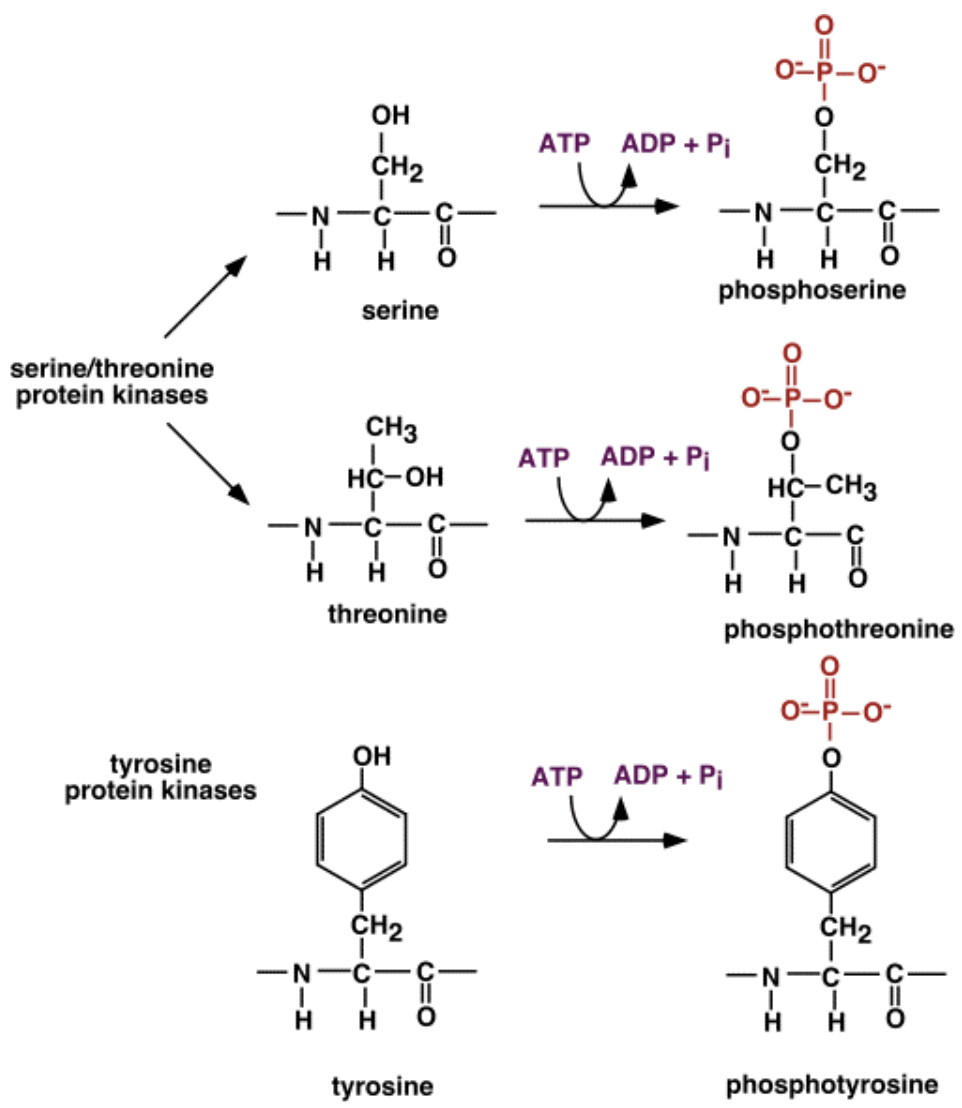

Figure 1-5: Phosphorylation of amino acids investigated in this work.

Serine/threonine kinases use ATP to incorporate the phosphoryl group (highlighted in red) to the side chain of serine/threonine residues. Another class of kinases (tyrosine kinases) is responsible for phosphorylation of bulky side chain of tyrosine, adapted from http://urei.bio.uci.edu/ hudel/bs99a/lecture26/lecture7_3.html

\subsubsection{Modulation of synaptic vesicle recycling by phosphorylation}

Protein phosphorylation is an important mechanism involved in regulation of neurotransmission. Advances in experimental tools such as genetic manipulation, expression and purification of proteins, viral transduction of primary neuronal cultures, development of phospho-specific antibodies, in vitro kinase assays and more recently the high throughput methods such as chip arrays and synthetic peptides as substrates for kinases and mass spectrometry (MS) based phosphoproteomics allowed us to identify many phosphorylated proteins in the nerve terminals and study the functional role of the phosphorylation sites in the recycling pathway. Most of the known nerve terminal phosphoproteins are involved in either exocytosis or endocytosis or both of the pathways such as cytoskeleton-related proteins. Here, I will discuss the known role of the phosphoproteins in each of the categories. 


\subsubsection{Exocytosis related phosphoproteins}

SNARE proteins are reported to be phosphorylated and modulate neurotransmission. Synaptobrevin is known to be phosphorylated by $\mathrm{Ca}^{2+} / \mathrm{calmodulin}$-dependent protein kinase II (CaMKII) at S61 (Hirling and Scheller, 1996) and casein kinase 2 (CK2) in vitro. This phosphorylation might regulate its interaction with other SNARE proteins (Nielander et al., 1995). Syntaxin 1 is phosphorylated at several sites. Syntaxin-1A phosphorylation at S14 and T21 by CK2 is known to enhance the release of glutamate from the nerve terminals via modulation of its interaction with Munc18 (Rickman and Duncan, 2010). Syntaxin-1A is also known to be the $\mathrm{Ca}^{2+}$-dependent substrate of death-associated protein (DAP) kinase at S188. This phosphorylation decreases its interaction with Munc18 (Tian et al., 2003). SNAP-25 is known to be phosphorylated by PKC at S187 resulting in the enhancement of its interaction with synaptotagmin 1 as well as SNARE complex formation and consequently, the release of vesicles (Nagy et al., 2002). In contrast, phosphorylation of SNAP-25 at T138 by protein kinase A (PKA) decreases the SNARE complex formation (Gao et al., 2016).

Besides the proteins of SNARE complex, other presynaptic proteins involved in the fusion of SVs are also reported to be phosphorylated. The majority of the phosphorylation sites modulate the interaction of the proteins and consequently neurotransmission. For instance, Munc18 is reported to be phosphorylated at several sites by different kinases. Phosphorylation at Y145 (by an unknown kinase) (Lim et al., 2013), S313 (by PKC) (Barclay et al., 2003) and T479 (by Dual-specificity tyrosine(Y)-phosphorylation-regulated kinase 1A (Dyrk1A)) (Park et al., 2012) is reported to increase the binding of Munc18 to syntaxin and therefore inhibit the SNARE complex formation. Also, it is reported that phosphorylation of Munc-18 at S241 (by extracellular signal-regulated protein kinase (ERK)) and at T574 (by cyclin-dependent kinase 5 (CdK5)) modulates the release of neurotransmitters via an unknown mechanism that is not related to the binding to syntaxin (Barclay et al., 2004, Schmitz et al., 2016). Interestingly, it is reported that activity-dependent phosphorylation of Munc18-1 by PKC leads to its redistribution. Upon stimulation, it is dispersed from the synapse and re-clusters in few minutes (Cijsouw et al., 2014).

As it was mentioned before, NSF is an ATPase that together with its cofactor a-SNAP disassembles the SNARE complex after fusion. It is known that NSF is phosphorylated at S460 
and T461 by PKCe and at T645 by Leucine-rich repeat kinase 2 (LRRK2), enhancing its ATPase activity (Chou et al., 2010, Belluzzi et al., 2016).

Synaptotagmin1 is involved in SV fusion and is the substrate of CaMKII and PKC at T112 (Hilfiker et al., 1999) and CK2 at T125 and T128 (Davletov et al., 1993). Phosphorylation of T112 is known to enhance the potentiation after frequent stimulation (de Jong et al., 2016). Complexins are also known to be phosphorylated at S115 in the C-terminal region by CK2. Phosphorylation of this site increases the complexin binding to SNARE complex (Shata et al., 2007, Malsam et al., 2009). Moreover, it has been shown that activity-dependent phosphorylation of complexin in Drosophila enhances the spontaneous release of neurotransmitters (Cho et al., 2015).

The components of the active zone are also reported to be heavily phosphorylated. RIM as the central organizer of the active zone, , has been reported to be phosphorylated at several sites. It is known that it is phosphorylated by CaMKII at S241 and S287, resulting in binding to the adaptor protein 14-3-3 (Sun et al., 2003). The PKA-dependent phosphorylation of RIM at S413 was initially suggested to trigger long-term plasticity (LTP) (Lonart et al., 2003). However, later it was revealed that it is not a critical factor for the LTP (Kaeser et al., 2008). Two other phosphosites of RIM (S447 and S745) are phosphorylated by ERK2 via Brainderived neurotrophic factor (BDNF) pathway and are involved in synaptic plasticity (SimsekDuran and Lonart, 2008).

The two giant proteins of the active zone, bassoon and piccolo, are known to be heavily phosphorylated. However, the functional relevance of these phosphorylation mostly unclear. For instance, phosphorylation of bassoon at S2844 is known to play a role in its binding to 14-3-3 protein (Schroder et al., 2013).

Munc13 is known as the priming factor of the active zone and a substrate of PKC. Also, it is reported that Munc13 undergoes conformation changes upon binding to DAG (the activator of PKC) and triggers potentiation of synaptic transmission in hippocampal neurons (de Jong et al., 2016).

Voltage-gated calcium channels are known to be regulated by phosphorylation. Phosphorylation N-type calcium channels by CdK5 at the C-terminal enhances their interaction with RIM, so they are clustered more efficiently in the active zone and therefore neurotransmitter release is enhanced ( $\mathrm{Su}$ et al., 2012). P/Q-type channels are also 
phosphorylated by CaMKII at the C-terminus that modulates their activity (Magupalli et al., 2013). It is known that both $\mathrm{N}$-type and P/Q-type channels interact with the SNARE protein via a region in cytoplasmic linker connecting domains II and III of their $\alpha 1$ subunits called synaptic protein interaction (synprint) site. Interestingly, this region is known to be the substrate of PKC and CaMKII and phosphorylation inhibits their interaction with the SNARE proteins (Yokoyama et al., 1997, Degtiar et al., 2000, Jarvis and Zamponi, 2001).

\subsubsection{Endocytosis related phosphoproteins}

Phosphoproteins involved in the clathrin-mediated endocytosis of SVs are categorized in a group called 'dephosphins'. Members of dephosphins are diverse with regard to their structure and function in different steps of SV endocytosis. Nevertheless, they share one characteristic: They undergo a cycle of phosphorylation and dephosphorylation during a round of endocytosis. Upon action potential and influx of $\mathrm{Ca}^{2+}$, they are dephosphorylated by a $\mathrm{Ca}^{2+} /$ calmodulin-dependent phosphatase called Calcineurin, and rephosphorylated by CdK5 during the resting condition. Members of this group are dynamin, the scaffold proteins including amphiphysin 1 and amphiphysin2, the PtdIns(4,5)P phosphatase, synaptojanin, the adaptor proteins, epsin, eps15, and AP180 (Cousin et al., 2001, Samuels and Tsai, 2003). Several studies have reported that phosphorylation of CME related proteins inhibits their protein-protein interaction with each other. For instance, interaction of AP-2 with clathrin (Wilde and Brodsky, 1996), AP-2 with AP-180 (Hao et al., 1999), eps15-Epsin complex with AP-2 (Chen et al., 1999), binding of dynamin and synaptojanin to SH3 domain of amphiphysin1, and amphiphysin1-AP-2 binding (Slepnev and De Camilli, 2000) are inhibited by phosphorylation.

\subsubsection{Cytoskeleton related phosphoproteins}

Three main cytoskeleton filaments are present in the nerve terminal: actin filaments, microtubules, and neurofilaments. It is known that actin filaments are closer to the active zone whereas the microtubules do not reach the active zone (Doussau and Augustine, 2000). Since the role of actin filaments and their associated proteins in neurotransmission has been studied more comprehensively, I will focus on them in the rest of this section. Actinassociated phosphoproteins are known to modulate the release of glutamate via different mechanisms. For instance, synapsin1 that links synaptic vesicles to actin filaments is known 
to be phosphorylated by several kinases. It is phosphorylated at S9, S556, and S603 by CaMKII, at S62, S67, S549, and S551by CdK5 and MAPK (Cesca et al., 2010), and at T301 by tyrosine kinase Src (Onofri et al., 2007). Interestingly, phosphorylation of synapsin at different sites has diverse effects on its actin-binding feature. Phosphorylation at S62, S67, S549, and S551 decreases, whereas phosphorylation at S9, S556, and S603 increases the actin-binding. Overall, it is known that upon elevation of the $\mathrm{Ca}^{2+}$ concentration, synapsin phosphorylation increases vesicle mobility and release of neurotransmitters (Cesca et al., 2010).

Myosins are actin-based motor proteins and some of myosin isoforms are present in the nerve terminals. It is known that myosin $\mathrm{V}$ is a motor protein that binds to SVs and facilitates their movement along the actin filaments. Binding of myosin $\mathrm{V}$ to synaptic vesicles is $\mathrm{Ca}^{2+}$ dependent and occurs through synaptobrevin and synaptophysin (Prekeris and Terrian, 1997). Another member of the myosin family, non-muscle myosin II, has also been shown to modulate synaptic transmission (Seabrooke and Stewart, 2011). The regulatory light chain of myosin II is phosphorylated by Myosin Light Chain Kinase (MLCK) at T18 and S19, which turns on its ATPase activity (Betapudi et al., 2010, Somlyo and Somlyo, 2003). In contrast, phosphorylation at S1, S2, and S3 amino acids by PKC has the opposite effect and turns off myosin II ATPase activity (Beach et al., 2011). Myosin II is also known to be phosphorylated by CK2 and PKC at heavy chain, regulating its assembly and localization (Betapudi, 2014). Reorganization of actin filaments has a role in the regulation of the neurotransmission. For instance, depolymerization of actin filaments enhances the vesicle secretion in chromaffin cells (Zhang et al., 1996). Therefore, actin-associated phosphoproteins such as cofilin, drebin, and spectrin that are involved in actin reorganization, might have an indirect role in neurotransmission.

\subsubsection{Kinases and phosphatases of the nerve terminal}

Phosphorylation and dephosphorylation of presynaptic proteins mediated by kinases and phosphatases present in the nerve terminal. Ser/Thr and Tyr kinases were initially discovered in the 1950s and 1980s, respectively. Kinases are the enzymes that transfer the $\gamma$-phosphoryl group of the ATP to the residues of amino acids (Figure 1-6). Exploring the

human genome, researchers identified 478 eukaryotic protein kinases that can be 
categorized into 7 families (CMGC, AGC, CAMK, CKI, STE, TKL, and TK) and 40 atypical kinases (Manning et al., 2002). Investigation of mouse genome revealed 549 kinases, majority of which are orthologues for human kinases (Caenepeel et al., 2004). Kinases recognize their targets physiologically via two mechanisms. First, via recognition of the consensus phosphorylation sequence by their active sites. Second, via interaction with the substrate at a site distant from the kinase catalytic site or the substrate phosphorylation site (Cheng et al., 2011).

Phosphatases are the enzymes that remove the phosphate group from the amino acids. 199 phosphatases have been reported in human that are classified into 6 families based on catalytic domain sequence similarity (Sacco et al., 2012). Ser/Thr phosphatases are not known to recognize a consensus sequence motif in their substrate. However, Tyr phosphatases are known to have a weak preference for phosphorylated tyrosine residues in the specific sequence of the target (Flint et al., 1997). Following in this section, I will discuss the regulatory mechanism of the most studied kinases and phosphatases present in the nerve terminals.

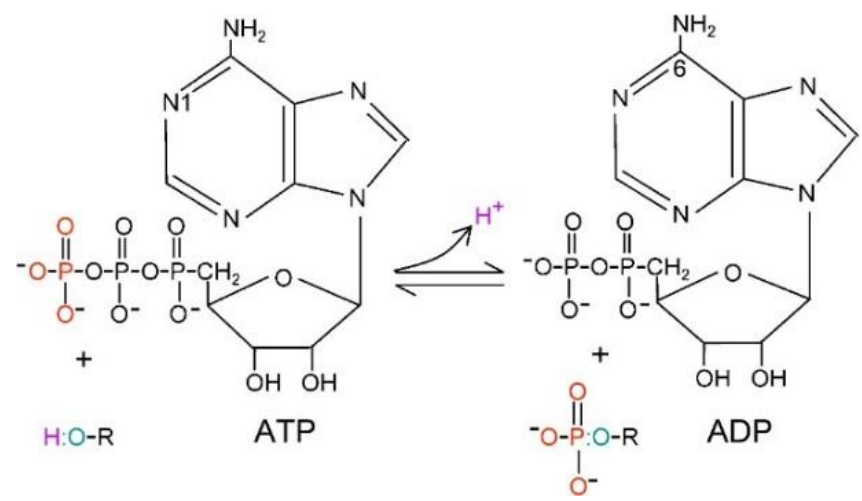

Figure 1-6: The protein kinase reaction.

In the reaction of protein phophorylation, kinases transfer $\gamma$-phosphoryl group from ATP to the hydroxyl group of the protein substrate (HO-R), producing the phosphoproteins and the ADP, adapted from (Roskoski Jr, 2012)

\subsubsection{Calcium/calmodulin-dependent protein kinase II (CaMKII)}

CaMKII is known to be activated by $\mathrm{Ca}^{2+} /$ calmodulin and was discovered in 1981 (Kennedy and Greengard, 1981). It is highly abundant in the brain (1-2\% of the total protein) and is specifically enriched in the synapse, both in the pre and postsynaptic part (Lisman et al., 2002). CaMKIIa- and $\beta$ subunits are the most common isomers in the brain that together or alone make a dodecameric holoenzymes. Each subunit has the catalytic domain, an auto- 
inhibitory domain, a variable segment and a self-association domain (Figure 1-7). The catalytic domain has an ATP and the substrate binding site that catalyzes the transfer of the phosphoryl group from the ATP to the substrate. The auto-inhibitory domain has a region that is similar to the CaMKII substrate and act as a pseudosubstrate that binds to the active site and inhibits the kinase activity. $\mathrm{Ca}^{2+} /$ calmodulin binds to a region that has an overlap with the pseudosubstrate region, therefore prevents the inhibitory domain from binding to the active site of the enzyme. Furthermore, this binding leads to the exposure of T286 on the auto-inhibitory domain. T286 can be autophosphorylated by the neighboring subunit and upon phosphorylation makes CaMKII insensitive to the $\mathrm{Ca}^{2+}$ concentration and keeps it active even in the low concentration of the $\mathrm{Ca}^{2+}$. The self-association domain links the subunits together into holoenzyme (Lisman et al., 2002, Rellos et al., 2010). Two other autophosphorylation sites T305 and T306 in the calmodulin binding region inhibit binding of calmodulin upon phosphorylation. Therefore, upon dissociation of $\mathrm{Ca}^{2+} /$ calmodulin, T305 and T306 are phosphorylated, inhibiting re-association of calmodulin and subsequently the enzyme activity (Hanson and Schulman, 1992).

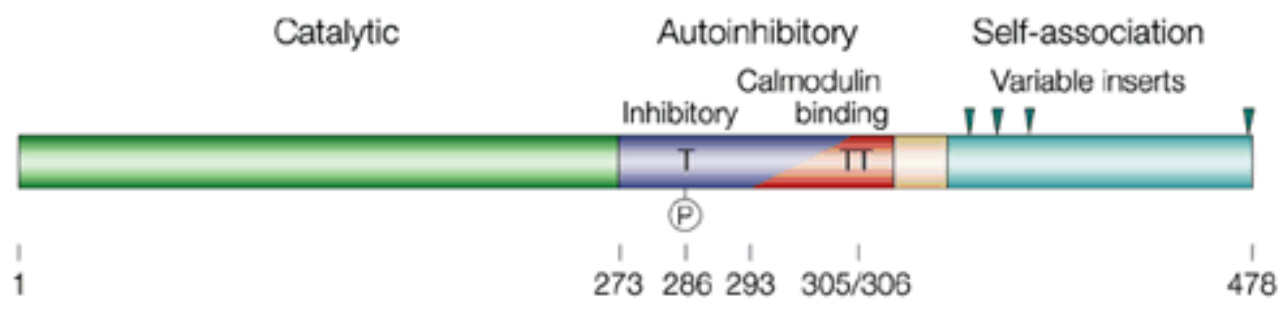

Figure 1-7: Functional domains in the primary structure of calcium/calmodulin-dependent protein kinase II (CaMKII).

Catalytic subunit uses ATP to phosphorylate substrates. However, it is inhibited by binding to the autoinhibitory domain. Upon binding $\mathrm{Ca}^{2+} /$ calmodulin to the auto-inhibitory domain, the inhibitory domain is displaced and the enzyme becomes active. Phosphorylation of three threonine residues (T) in this domain is important for regulation of CaMKII. Phosphorylation of T286 keeps CaMKII active even in low Ca ${ }^{2+}$ concentration. Phosphorylation of T305 and T306 inhibits calmodulin binding, therefore, auto-inhibits CaMKII activity, , adapted from (Lisman et al., 2002).

Besides CaMKII, other members of the CaMK family, for instance CaMKI and CaMKIV are also present in the nerve terminal. Unlike CaMKII, these kinases are present in monomeric form and have an activation loop phosphorylation site that upon binding to $\mathrm{Ca}^{2+} / \mathrm{CaM}$ is exposed to the upstream CaMK kinase. Upon phosphorylation of this site by CaMKK, these enzymes become active for a long period of time (in case of CaMKI, up to $1 \mathrm{~h}$ ). Interestingly, 
CaMKK/CaMKI cascades of phosphorylate the Mek/ERK kinases and activate them and plays a curtail role in long-term potentiation (LTP) of synapses (Wayman et al., 2008).

\subsubsection{Protein kinase A (PKA)}

PKA was discovered in 1969 in adipose tissue for the first time and was known to be activated by cyclic adenosine monophosphate (cAMP) (Corbin and Krebs, 1969). PKA has two catalytic subunits and two regulatory subunits. Upon binding of cAMP to the regulatory subunit, it undergoes a conformational change that inhibits its binding to the catalytic subunit (Elkins and Knapp, 2012), resulting in the activation of PKA. Therefore, the regulation of adenylate cyclase (the enzyme that catalyzes production of cAMP) has an important role in controlling PKA activity. Nine isoforms of adenylate cyclase have been identified in mammalian cells and it is known that they are regulated by $\mathrm{Ca}^{2+}$-signaling pathway components in opposite ways. For instance, they are inhibited by high concentration of free $\mathrm{Ca}^{2+}$ and activation of CaMKII, but, at the same time, are activated through binding to $\mathrm{Ca}^{2+} / \mathrm{CaM}$ complex and $\mathrm{PKC}$ phosphorylation (Halls and Cooper, 2011). Although the regulation of PKA by $\mathrm{Ca}^{2+}$ is very complex, overall, it has been reported that PKA gets activated upon influx of $\mathrm{Ca}^{2+}$ into the nerve terminal (Millan et al., 2003). Interestingly, PKA binds to a family of scaffold proteins called A-kinase anchoring proteins (AKAPs) that spatially restrict PKA close to the plasma membrane and SV fusion sites (Park et al., 2014). Additionally, PKA activity is regulated by phosphorylation of T197 in the activation loop of the catalytic subunit. Phosphorylation at this site facilitates the phosphate group transfer to the substrate by stabilizing the transition state through an electrostatic interaction (Cheng et al., 2006). It has been suggested that this site is phosphorylated via autophosphorylation or by phosphoinositide-dependent kinase-1 (PDK-1) (Moore et al., 2002).

\subsubsection{Protein kinase C (PKC)}

Protein kinase $\mathrm{C}$ is represented by a multigene family of kinases that are similar in the catalytic domain but are different in the regulatory domains. Based on their structure, PKC isoforms are categorized into three major groups: conventional PKCs (cPKC; consisting of $\alpha$, $\beta \mathrm{I}$, and $\beta \mathrm{II}$ isoforms), novel PKCs (nPKC; consisting of $\delta / \theta$ and $\varepsilon / \eta$ isoforms) and atypical PKCs (aPKC; consists of $\zeta$ and $\mathrm{l} / \lambda$ isoforms) (Figure 1-8). All PKC isoforms have a C1 domain that binds to diacylglycerol (DAG) and phorbol esters. However, only cPKC and nPKC have 
the ability to bind to DAG. cPKC and nPKC but not aPKC contain a C2 domain that only in case of cPKC functions as the $\mathrm{Ca}^{2+}$ sensors. Therefore, the $\mathrm{cPKC}$ are regulated both by the presence of DAG or phorbol esters and $\mathrm{Ca}^{2+}$. The nPKCs are regulated only by DAG or phorbol esters (Heemskerk et al., 2011) and aPKC do not require $\mathrm{Ca}^{2+}$ or DAG for activation and are regulated by phosphatidylinositol 3,4,5- $\mathrm{P}_{3}$ ( $\mathrm{PI}-3,4,5-\mathrm{P}_{3}$ ) and ceramides (Nakanishi et al., 1993). Similar to PKA, all the PKCs are also phosphorylated at the T residue (the exact site is different in the isomers) in their activation loops. Phosphorylation of this residue is necessary for the enzymatic activity of PKCs is known to be performed by PDK-1. Once PKCs become active, they bind to the Receptor for Activated C-Kinase (RACK-1) in order to be localized to distinct membrane microdomains (Heemskerk et al., 2011).

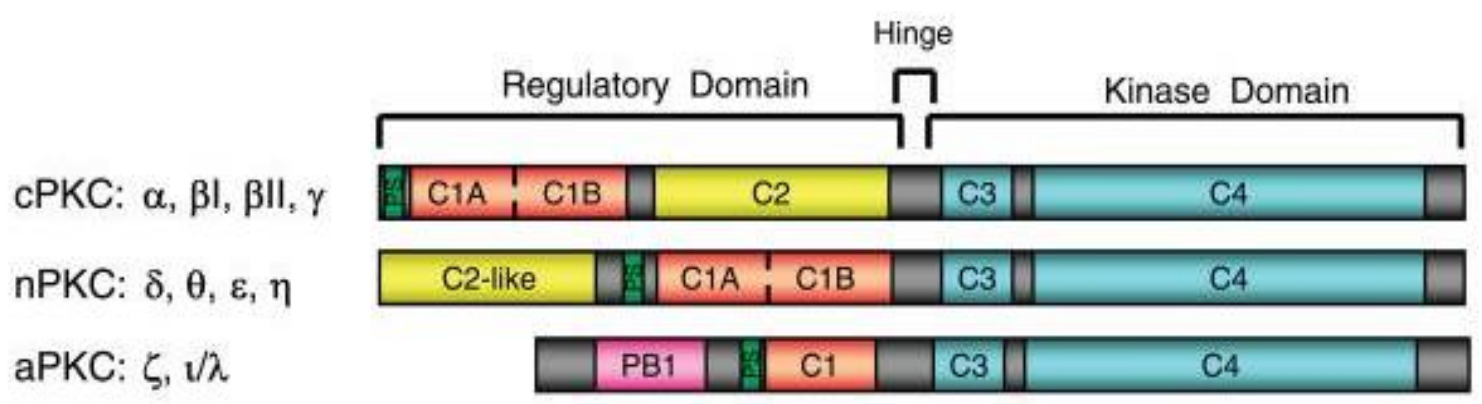

Figure 1-8: Functional domains in the primary structure of protein kinase C (PKC) isoforms All PKC isoforms have a conserved catalytic domain at the C-terminal, whereas regulatory domain is variable among them. Conventional PKC (cPKC) and novel PKC (nPKC) can be regulated by phorbol esters and diacylglycerol through the $\mathrm{C} 1$ domain. $\mathrm{cPKC}$ are also regulated by $\mathrm{Ca}^{2+}$ through $\mathrm{C} 2$ domain. $\mathrm{nPKC}$ despite having C2 domain are not regulated by calcium. In contrast to other isoforms, atypical PKC (aPKC) are regulated only by $\mathrm{PIP}_{3}$, and ceramides but not by phorbol esters or calcium ions, adapted from (Steinberg, 2008).

\subsubsection{Casein kinase 2 (CK2)}

CK2 is a heterotetrameric holoenzyme that consists of two catalytic ( $\alpha$ and $\alpha^{\prime}$ ) and a noncatalytic subunit ( $\beta$ ) and is classified as an atypical kinase (Venerando et al., 2014). It is known that CK2 phosphorylates proteins at the sites specified by acidic determinants (S/T$\mathrm{X}-\mathrm{X}-\mathrm{E} / \mathrm{D} / \mathrm{pS} / \mathrm{pT}$ consensus motif). The other characteristic of CK2 is that it is constitutively active in the cell and is ready to cooperate with different signals often triggered by an external stimulus (Lolli et al., 2012). Several mechanisms are suggested to regulate CK2 activity. In a very rare mechanism, CK2 molecules make an inactive polymer via a $\beta$ subunits and only the molecules that can escape from this supermolecule by an unknown mechanism become 
active. Phosphorylation is another mechanism that has been suggested as the regulatory mechanism for CK2. Phosphorylation by other kinases or possibly autophosphorylation has an inhibitory effect on the activity of CK2. It has been shown that treatment of CK2 by variety of phosphatases results in the enhancement of its kinase activity (Agostinis et al., 1987). Furthermore, it has been reported that inositol 1,3,4,5-tetrakisphosphate (IP4) and inositol hexaphosphate (IP6) are able to increase the catalytic activity of CK2 (Solyakov et al., 2004).

\subsubsection{Extracellular-signal Regulated Kinase 1/2 (ERK1/2)}

Extracellular-signal Regulated Kinase 1/2 (also known asMAPK1/3) represent a class of Ser/Thr kinases that are involved in Ras-Raf-MEK-ERK signal transduction cascade. MEK is a member of dual-specificity kinases that is able to phosphorylate both Ser/Thr and Tyr. It is known that MEK activates ERK1/2 by phosphorylation at T204/187 and then Y202/185. ERK1/2 is known as a proline-directed kinase that phosphorylates its substrates at P-XXXS/T-P consensus sequence (Roskoski Jr, 2012). It has been shown that the increase in cytosolic $\mathrm{Ca}^{2+}$ concentration increases Ras activity and consequently enhances activity of the ERK1/2.

\subsubsection{Cyclin-dependent kinase 5 (CdK5)}

CdK5 is the most abundant member of cyclin-dependent kinase family in the brain (Shah and Lahiri, 2014). Unlike other CdKs family members, CdK5 activity is not dependent on cyclinbinding but requires interaction partners called p35 and p39 (Tsai et al., 1994). Activated CdK5 auto-inhibits itself by phosphorylation of p35 at S8 and T138. Phosphorylation of p35 in these two sites leads to their rapid degradation (Wei et al., 2005) and allows CdK5 to regulate its own activity. It is known that T138 is dephosphorylated by PP1 and PP2A leading to more stable p35 (Kamei et al., 2007). Moreover, CdK5 can be phosphorylated by Abl, ephrin receptor A (EphA) and Fyn kinases, resulting in its activation (Zukerberg et al., 2000, Sasaki et al., 2002). CdK5 has several targets in the presynapse. It phosphorylates synapsin1 at S549 and decreases its binding to actin filaments while binding to SVs remains intact (Jovanovic et al., 2001). As it was mentioned above, it phosphorylates Munc18 and decreases its binding to syntaxin (Barclay et al., 2003). However, the most-studied role of CdK5 in neurotransmission is the re-phosphorylation of dephosphins in the resting condition. It is known that CdK5 phosphorylates dynamin1 at S778 (Clayton et al., 2010), as well as 
amphiphysin at S261 and T310 (Liang et al., 2007, Tomizawa et al., 2003a) and synaptojanin at S1144 (Lee et al., 2004).

\subsubsection{Glycogen synthase kinase 3 (GSK-3)}

GSK-3 was initially identified as a kinase that phosphorylates glycogen synthase in liver (Rylatt et al., 1980). It exists in two different isoforms: GSK-3 $\alpha$ and $\beta$. It is known that GSK-3 is constitutively active and is negatively regulated by phosphorylation at the $\mathrm{N}$-terminal (at S21 in GSK-3 $\alpha$ and at S9 in GSK-3 $\beta$ ) (Eldar-Finkelman, 2002). Upon increase of cytosolic $\mathrm{Ca}^{2+}$ concertation, the S9/S21 inhibitory site is phosphorylated by CaMKII (Song et al., 2010). GSK$3 \beta$ is also phosphorylated at S34 by ERK1/2, which does not directly inhibit the kinase activity. However, it increases the capacity of S9/S21 to be phosphorylated. In contrast, the tyrosine phosphorylation at 279 of GSK-3 $\alpha$ or 216 of GSK-3 $\beta$ are known to enhance the activity of the GSK-3. One interesting feature of GSK-3 is that some of its substrates do not need to have a very specific sequence, whereas they are required to be phosphorylated by another kinase, known as 'priming phosphorylation', so they can become the GSK-3 substrate (Medina and Wandosell, 2011). GSK-3 is detected in the nerve terminals and phosphorylates P/Q-type calcium channels to decrease their activity (Zhu et al., 2010). The phosphorylation of GSK-3 at S9/S21 inhibitory site happens only upon intense stimulation of neurons. It is known that GSK-3, together with CdK5, re-phosphorylates dephosphins after stimulation in the resting condition. In fact, CdK5 does the priming phosphorylation and GSK-3 continues phosphorylation of the CME component (Smillie and Cousin, 2011). It is known that GSK-3 $\alpha$ and GSK-3 $\beta$ are dephosphorylated by PP2A and PP1, respectively, that leads to their activation (Hernández et al., 2010).

\subsubsection{Protein phosphatase 1 (PP1)}

The activity of PP1 is largely regulated by the protein-protein interactions that affects the catalytic characteristics of PP1 (gating mechanism) and localization of the enzyme. Two regulatory proteins are known to have a role in the gating mechanism: Inhibitor- 1 that was found initially in the middle of the 70s (Huang and Glinsmann, 1975) and the Dopamine- and cAMP-Regulated Phospho Protein 32 (DARPP-32) (Walaas et al., 1983). Both of the regulatory proteins are phosphorylated by PKA, making them potent inhibitors of PP1. Protein phosphatase 2B is known to dephosphorylate these sites and decreases their PP1 
inhibitory function. PP1 is also regulated by its localization via Neurabin. Neurabin is a regulatory protein of PP1 and it links PP1 to actin filaments. Phosphorylation of Neurabin at S100 by CaMKII (Colbran, 2004) and at S461 by PKA significantly reduces its actin-binding. Consequently, PP1 diffuses from the nerve terminal which is actin-rich region (McAvoy et al., 1999).

\subsubsection{Protein phosphatase 2A (PP2A)}

PP2A is known to be pharmacologically similar to PP1, and it is downregulated by the same inhibitors such as Okadaic acid and Calyculin A (Winder and Sweatt, 2001). It is known that phosphorylation of PP2A at B' $\alpha$ regulatory subunit by CaMKII decreases its phosphatase

activity (Fukunaga et al., 2000). In contrast, phosphorylation of PP2A regulatory subunit delta at S567 by PKA enhances its activity (Ahn et al., 2007).

\subsubsection{Protein phosphatase 2B (PP2B, Calcineurin)}

PP2B (also known as Calcineurin) consists of a catalytic subunit (A) and a regulatory subunit (B). It is known that high concentrations of $\mathrm{Ca}^{2+}$ ions are the main regulator of Calcineurin. $\mathrm{Ca}^{2+}$ ions bind to the $\mathrm{B}$ subunit and displace the auto-inhibitory region of PP2B, resulting in activation of Calcineurin. However, the activity is enhanced significantly when the complex of $\mathrm{Ca}^{2+}$ /Calmodulin binds to the regulatory B subunit (Perrino et al., 1995). Besides $\mathrm{Ca}^{2+}$ ions, PP2B is regulated by a family of proteins called Calcipressins. It is known that Calcipressin-1 is phosphorylated at S108 and S112 by GSK-3 $\beta$ and MAPK, respectively. Phosphorylation turns Calcipressin-1 into an inhibitor of PP2B. Also, it decreases the half-life of Calcipressin1 (Ma et al., 2012).

\subsubsection{Modulation of synaptic vesicle pools via kinases/phosphatases}

Synaptic strength is known to be precisely tuned in the nerve terminal. This is achieved by regulation of SV pool sizes and the availability of SVs to be released. In this section, I will discuss the role of kinases/phosphatases on regulation of SV pools.

Vesicles are known to be clustered by synapsin in the reserve pool (Hilfiker et al., 1999) and upon phosphorylation by CaMKII, the clusters will be released and SVs are more available for the release. However, other studies revealed that synapsin does not specifically distinguish 
vesicles of the reverse pool from the other pools, and lack of synapsin affects both recycling and RRP pools (Ryan et al., 1996, Mozhayeva et al., 2002). Recently, it has been shown that the phosphorylation of synapsin 1 at $S 549$ by CdK5 increases the ratio of reserve pool to the recycling pool via enhancing its interaction to the actin filaments (Verstegen et al., 2014).

The CdK5/Calcineurin system is another mechanism that has been proposed to regulate the ratio between recycling and reserve pool of vesicles (Kim and Ryan, 2010, Kim and Ryan, 2013). Cdk5 is known to facilitate the exocytosis via phosphorylation of Munc18 (Fletcher et al., 1999) and septin5 (Taniguchi et al., 2007). Also, the CdK5/Calcineurin balance has been shown to regulate the release probability of SVs by controlling the influx of calcium into the nerve terminal (Kim and Ryan, 2013). CdK5 and Calcineurin also have an opposite effect (phosphorylation and dephosphorylation) on the endocytosis-related proteins, resulting in regulation of the SV recycling pathway (Cousin and Robinson, 2001). CdK5/Calcineurin activity also determines which fraction of SVs is categorized as recycling or reserve pools (Fassio et al., 2016). Moreover, PKC and PKA were also suggested to modulate the size of synaptic vesicle pools. PKC increases the size of the readily releasable pool (Stevens and Sullivan, 1998, Fioravante et al., 2011) and PKA increases the recruitment of SVs from reserve pool to the recycling pool (Kuromi and Kidokoro, 2003, Kuromi and Kidokoro, 2000).

\subsection{Mass spectrometry (MS)-based phosphoproteomics}

\subsubsection{Protein Identification}

Traditionally, specific proteins could only be detected in complex mixture of extracted proteins using specific affinity ligands such as antibodies. These methods are limited to proteins for which specific antibodies are available and thus an overview of the protein composition of a complex sample could not be obtained. The only alternative method was to obtain sequence information for instance by $\mathrm{N}$-terminal sequencing using Edman method (Edman, 1949). However, this method requires (i) the separation (purification) of the protein from other constituents (e.g. by 2D-electrophoresis), and (ii) an unblocked N-terminus. In the last decade, the field has been revolutionize by the developments in mass

spectrometry. Presently, it is possible to identify thousands of (phospho)proteins in a single experiment (Lemeer and Heck, 2009, Kelstrup et al., 2014, Ludwig et al., 2015) by MS-based techniques. A general workflow of a typical shotgun proteomics experiment consists of the 
flowing (Figure 1-9): (1) protein extraction, (2) protein fractionation/enrichment, (3) protein digestion, (4) fractionation/enrichment on the peptide level, (5) MS analysis of the peptides, followed by MS/MS sequencing of the selected peptides and (6) peptide/protein identification (database search).
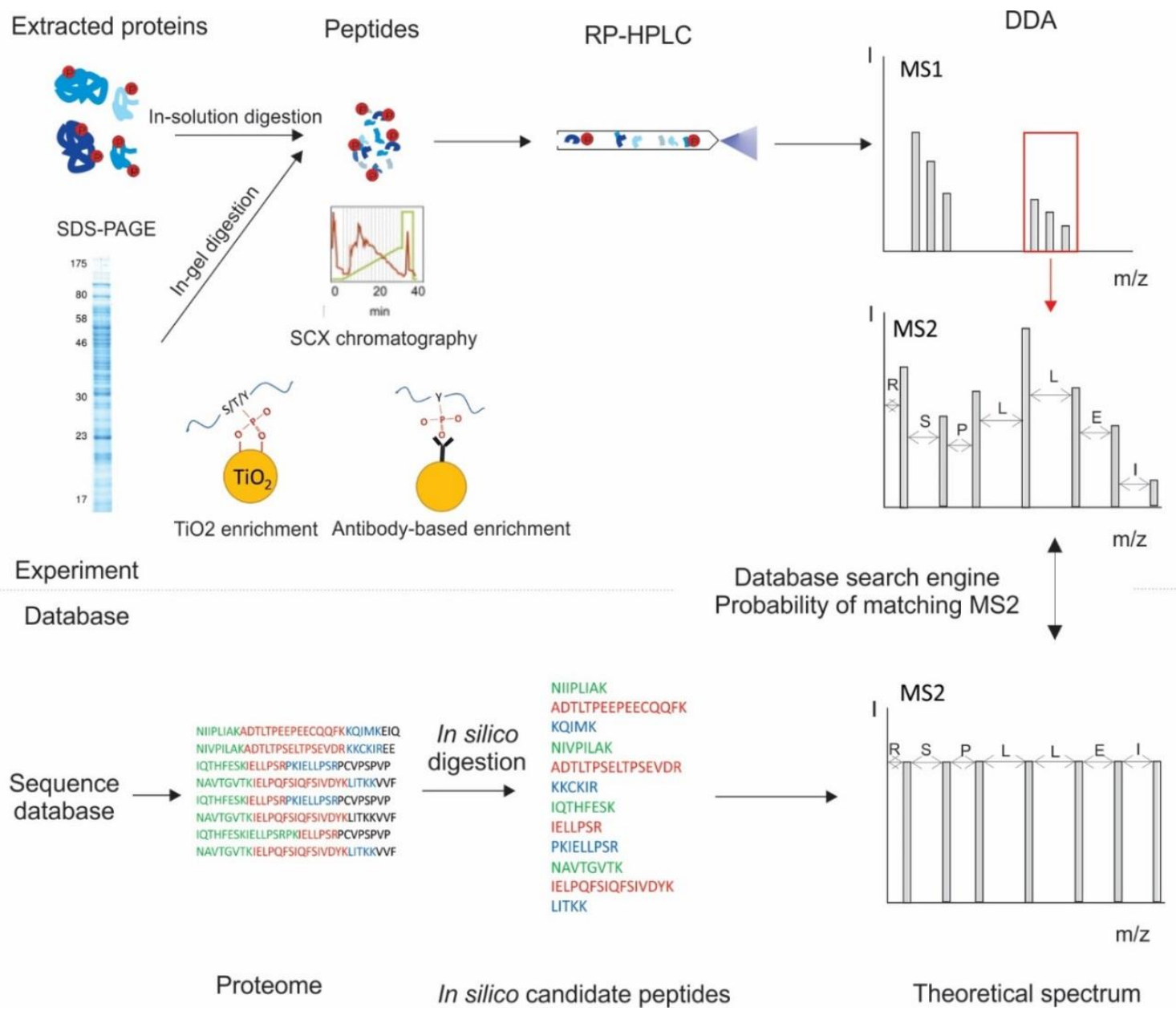

Figure 1-9: The general workflow of a shotgun (phospho)proteomics

Extracted proteins from the cell or tissue can be subjected to a fractionation method (e.g. SDS-PAGE base on molecular size) at the protein level or after digestion with an endoprotease (most commonly used, trypsin) at the peptide level (e.g. strong cation exchange (SCX)). Modified peptides (here we focus on phosphorylation) can be enriched (e.g. titanium dioxide $\left(\mathrm{TiO}_{2}\right)$ enrichment, phospho-tyrosine immunoprecipitation). Peptides are subjected to reverse phase high-performance liquid chromatography (RP-HPLC) that is connected to mass spectrometer to be further fractionated prior to entering the instrument. Eluted peptides are ionized by electrospray ionization and are analyzed online by mass spectrometer. In data-dependent acquisition (DDA) mode, a survey scan (MS1) will measure the mass to charge ratio $(\mathrm{m} / \mathrm{z})$ of all the peptides entering into the instrument at a particular moment and then the most abundant precursors are selected for the fragmentation and generation of MS2 spectra. The (phospho)peptides are identified by matching the experimentally acquired MS2 scans and the theoretical MS2 scans generated by search engines software via in silico digestion of desired proteins sequences. 
Proteins are commonly extracted from the cell or tissue by physical disruption or application of lysis buffers containing detergents to rupture the cell membranes. Extracted proteins are often fractionated to decrease the complexity of the sample. One of the most common methods is SDS-PAGE to separate proteins based on their molecular weight.

The next step in bottom-up proteomics is the digestion of proteins into peptides that can be done either in-solution, on the filter (Wisniewski et al., 2009) or in-gel (Shevchenko et al., 1996). The most common protease that is used for this purpose is trypsin. Trypsin cleaves exclusively at the C-terminal of arginine $(\mathrm{R})$ and lysine $(\mathrm{K})$ amino acids and is suitable for proteomics studies due to two main reasons: First: the frequency of $\mathrm{R}$ and $\mathrm{K}$ in the proteome of organisms is optimal to generate 7-20 amino acid peptides that are suitable for fragmentation by tandem MS/MS analysis. Second, it leaves a highly basic residue at the Cterminal of the peptides resulting in efficient ionization and production of high mass $y$-ion series spectra upon fragmentation which in turn makes spectra more informative and easy to interpret (Olsen et al., 2004, Medzihradszky and Chalkley, 2015). Other proteases such as Lys-C and chymotrypsin are also used but less frequently to improve the coverage of proteome and PTM identification (Giansanti et al., 2016). Fractionation/enrichment can also be done at the level of peptides. This is more common in the case of PTM identification since modifications are more exposed at the peptide level and can be enriched more efficiently. Different approaches have been employed for fractionation and enrichment at the peptide level. They include: ion exchange chromatography, metal affinity chromatography, immunoprecipitation by antibodies (e.g. to enrich phospho-tyrosines) or affinity-base enrichment such as lectins for glycosylated peptides. These methods are reviewed in various recent publications (Macek et al., 2009, Nilsson, 2012, Zhang et al., 2013b, Zargar et al., 2015). Before analysis by mass spectrometer, the peptides are fractionated by reverse-phase high performance-liquid chromatography (HPLC), usually connected on-line to the mass spectrometer instrument. The peptides are separated based on their hydrophobicity and enter the instrument gradually, being analyzed continuously by consecutive scans in the mass spectrometer. Below I will discuss several aspects of MS analysis as well as peptide/protein identification in more detail. 


\subsubsection{Tandem mass spectrometry}

The identification of proteins by MS relies on the identification of at least one unique peptide derived from a particular protein. Peptide analysis by a mass spectrometer determines the mass/charge $(\mathrm{m} / \mathrm{z})$ ratio and the intensity of the peptide in the gas-phase. Although this information is valuable, it is not enough for the identification of peptides. The primary amino acid sequence of the peptide provides sufficient and necessary information for the peptide identification. The method that is widely used for the peptide sequence identification is 'Tandem mass spectrometry' (also known as MS/MS). In this method, the $\mathrm{m} / \mathrm{z}$ ratio of all the peptides eluting at a particular moment from the chromatography separation is measured in the first scan (also known as the survey scan or MS1). Then, depending on the speed of the instrument, a limited number of the peptides (commonly between 10-30) with the highest intensities are selected for the fragmentation. The peptides selected for the fragmentation are called the precursor ions. Precursor ions are fragmented to yield smaller parts (product ions). The mass of the product ions is determined in the second scan (MS2). In modern mass spectrometers, all the processes of the precursor selection and fragmentation occur in an automated fashion, called the 'Data-Dependent Acquisition (DDA)' mode. The DDA acquisition is biased towards the identification of highly abundant peptides (Steen and Mann, 2004). This limitation makes the identification of phosphopeptides more challenging since often phosphorylation occurs at sub-stoichiometric levels. However, improvement in the MS instrumentation and phosphopeptides sample preparation can partially overcome these limitations (Olsen and Mann, 2013).

\subsubsection{Sample ionization}

Since mass spectrometers are able to measure the mass of charged species, the peptides have to be ionized before entering the instrument. However, the ionization of large biological molecules in the gas phase is not trivial. Two methods have been developed in order to ionize the biomolecules while leaving them intact during ionization (soft ionization): electrospray ionization (ESI) (Fenn et al., 1989) and matrix-assisted laser desorption ionization (MALDI) (Tanaka, 1988). Since, these methods revolutionized the field of the mass spectrometry and proteomics, their inventors (John B. Fenn and Koichi Tanaka) were awarded the chemistry Nobel Prize in 2002. In ESI, a high electrostatic voltage is set between the tip of the capillary needle and the entrance of the mass spectrometer. In the LC-ESI while the sample is eluting 
from the chromatography column and passing through the needle, they are sprayed in form of small charged droplet. As the liquid is evaporating the charge concentration at the surface increases, which leads to dissociation of the droplet and a decrease in the size of the droplet. This process goes on until the liquid completely evaporates, resulting in charged species. However, the exact mechanism leading to the ionization of the analyte by ESI is debated (Konermann et al., 2013). In MALDI, the analyte molecules are co-crystallized together with a chemical matrix which is typically a benzoic acid derivative and is able to absorb UV. Then a laser pulse is fired to the matrix, resulting in production of ionized matrix molecules, protonated and deprotonated matrix molecules, matrix clusters and nanodroplets. These species are involved in the transfer of protons to the analyte molecules and creation of charged analytes in the gas phase through the mechanism that is still under debate (Shevchenko et al., 2000, Lu et al., 2015).

The advantage of ESI over MALDI is that ESI tends to produce multiple charged species. This is why, very large proteins can appear at lower $m / z$ values by ESI fragmentation. Therefore, theoretically it is possible to analyze unlimited mass range of proteins with ESI (El-Aneed et al., 2009). Also, ESI implies use of solvated samples, whereas solid state samples are utilized for MALDI. Therefore, ESI is more suitable to be combined with a reverse-phase liquid chromatography to separate peptides based on their hydrophobicity in a complex sample with simultaneous analysis upon their elution from the column. Recently the more advanced chromatography methods (nano-ESI) utilizing slower flow rates (a few hundred $\mathrm{nL} / \mathrm{min}$ ) have led to higher ionization efficiency and better sensitivity (Schmidt et al., 2003).

\subsubsection{Peptide sequencing}

As mentioned above, in the tandem mass spectrometry after the survey scan, the selected precursors are fragmented yielding the product ions. The product ions generated by fragmentation are categorized, based on the location of breakage of the peptide backbone, into three groups: 1) a- and x-ions, 2) b- and y-ions and 3) c- and z- ions (see Figure 1-10). The most widely used fragmentation method for the identification and quantification of the proteome is Collision-Induced Dissociation (CID). In this method, the precursors are collided multiple times with a neutral gas molecule such as helium, nitrogen or argon. In Thermo Scientific mass spectrometers, which were used in this project, the CID occurs in the ion trap mass analyzer. Consequently, the precursors break down along the peptide backbone and at 
peptide bonds. The ion trap analyzer has a low mass cut-off that cannot detect the ions with $\mathrm{m} / \mathrm{z}$ less than $30 \%$ of the precursor (Zhang et al., 2005). More recent fragmentation method is High-energy Collisional Dissociation (HCD). In contrast to CID, in Thermo Scientific mass spectrometers, HCD occurs in a collision cell rather than the ion trap. Then they are accumulated in a C-trap, and transferred to be analyzed by a high resolution mass analyzer, such as orbitrap. Combination of HCD fragmentation and utilization of orbitrap has advantages. It does not have the low cut-off limitation and the resolution of resulting spectrum is higher since it generates more product ions leading to a higher quality MS/MS spectra.

Both CID and HCD fragmentation generate the $b$ - and $y$ - ions. Another type of fragmentation that is known to be complementary to CID and HCD is the electron transfer dissociation (ETD). In this method, the precursors interact with an electron donor resulting in an electron transfer to the peptide and cleavage of the peptide backbone and generation of the c- and zfragments ions. It is known that ETD particularly works better on highly charged states of peptides (Guthals and Bandeira, 2012). The advantage of ETD in phosphoproteomics is that it does not induce the neutral loss of phosphoric acid as it happens via CID fragmentation and leaves the phosphorylation modification intact (Quan L, 2013).

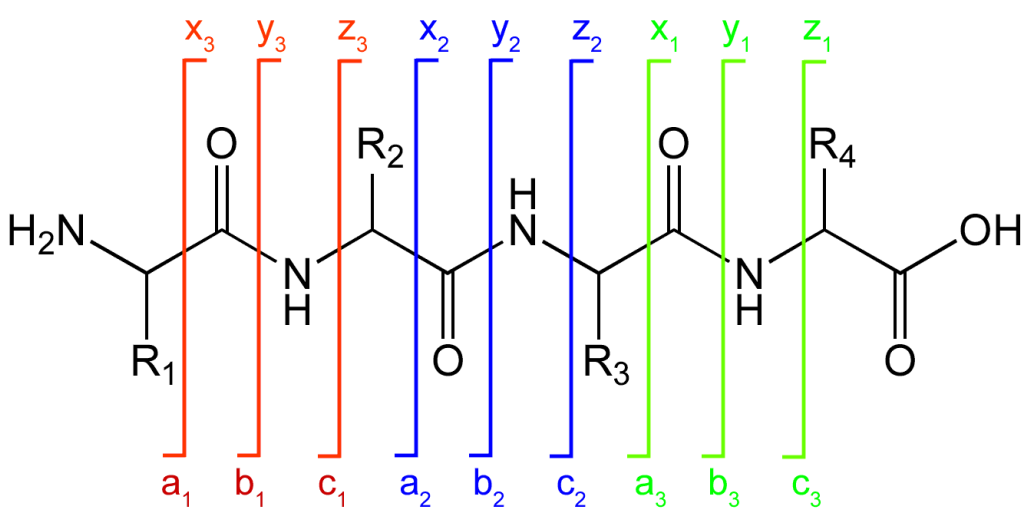

Figure 1-10: Nomenclature of fragment ions.

Fragments ions are grouped based on the location of the fragmentation on the peptide backbone. A- and x-ions are the result of breakage of alkyl-carbon bonds. B- and y-ions are generated after the breakage of peptide bond and c- and z- ions are formed after breakage of amino-alkyl bond. A-, b-, c- ions contain the N-terminal of the peptide whereas $\mathrm{x}-, \mathrm{y}$ - and $\mathrm{z}$ - ions contain the C-terminal of the peptide (Roepstorff and Fohlman, 1984). 


\subsubsection{Mass spectrometry instrumentation}

In the tandem mass spectrometers, the first and the second mass scan occur in more than one mass analyzer. These instruments are often hybrid mass spectrometers which combine different mass analyzers to obtain better performance. So far, different mass analyzers have been developed with different characteristics in mass accuracy, dynamic range, mass scan speed and the resolution (Glish and Burinsky, 2008). The most common mass analyzers are quadrupole, ion traps, time-of-flight and orbitrap. Most hybrid analyzers are ion traporbitrap, quadrupole-TOF, and quadrupole-orbitrap. The Q-Exactive instrument that was mostly used in this study is a hybrid quadrupole-orbitrap mass spectrometer that combines the high-performance precursor selection feature of quadrupole to the high-resolution and accurate-mass detection of orbitrap. Figure 1-11 demonstrates the schematic view of a QExactive HF instrument.

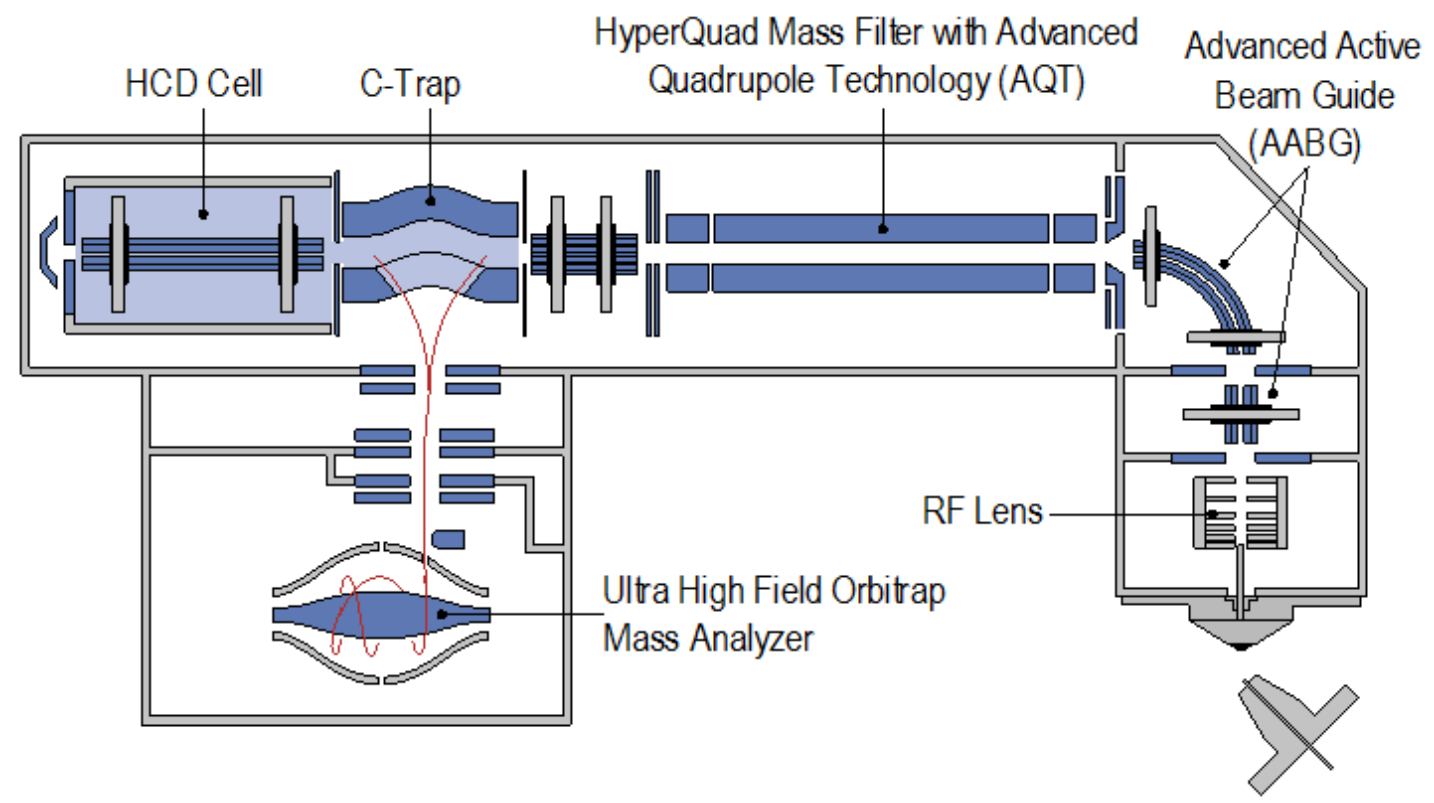

Figure 1-11: The schematic overview of Q-Exactive HF instrument.

$\mathrm{RF}$ lens increases the efficiency of ion capture, focuses and transmits them. AABG prevents the neutral particles from entering the quadrupole and, consequently, decreases the noise. Quadrupole is used to select the precursor ions and also to enable the parallel data acquisition. Precursor ions are transferred to HCD cell where they are fragmented efficiently to generate product ions. Then the product ions are collected in C-Trap which focuses ions efficiently and injects them to the orbitrap mass analyzer. Orbitrap detects the mass of ions accurately with a high resolution. Adapted from Planet Orbitrap manual (http://planetorbitrap.com/qexactive\#.V_YpzXq8Nlw).

In the recent years, the orbitrap mass analyzers have significantly contributed to the proteomics field. Therefore, I will devote the rest of this section to the characteristics of the orbitrap mass analyzers. The orbitrap analyzer consists of three electrodes. One is located in 
the center and two outer electrodes that have cup shape facing each other. When the voltage is applied between the outer and central electrodes it produces an electrical field that is strictly linear along the axis. Simultaneously, the radial component of the electrical field attracts the ions to the central electrode. When ions are injected in the volume between outer and central electrodes, they are trapped in the electrostatic field between the electrodes and rotate and oscillate around the central electrode. Using the image current detection and Fourier transformation algorithm, the axial frequency movements of ions are converted to the $\mathrm{m} / \mathrm{z}$ ratio (Zubarev and Makarov, 2013). Orbitraps have high mass resolution, mass accuracy, dynamic range, and upper mass limit (Scigelova and Makarov, 2006). Recently, the invention of high-field (HF) orbitrap analyzers that have smaller trap size has increased the resolution of these series of mass analyzers (Makarov et al., 2009).

\subsubsection{Peptide and protein identification}

Improvements in the high-throughput proteomics and generation of several thousands of spectra in one experiment rendered the annotation of spectra unfeasible. Several search engines and software have been developed to identify and reliably quantify the peptides/proteins automatically and also to localize the PTM on the peptides. The general workflow of the search engines relies on the generation of a library with theoretical MS2 spectra in silico based on the sequence of desired protein(s) or complete genome of an organism as well as the specific endoproteases and probable quantitation labels and/or PTM(s). Then they compare the experimental MS2 spectra with the theoretical MS2 spectra. For each experimental MS2 spectrum several theoretical spectra can be assigned with a score that is calculated based on the quality of the match between the theoretical and the experimental spectrum. Peptides with the highest score value are then identified (Paulo, 2013). Since this workflow is prone to yield false positive identification of peptides, a statistical approach called false discovery rate (FDR) is used to filter out false positive results. FDR is calculated by matching the experimental spectra with a decoy database that can be the reverse or scrambled sequence of the desired proteins. Commonly, the 1\% FDR is acceptable for the shotgun experiments (Elias and Gygi, 2007). In this project, I have used the freely available software called MaxQuant which contains a search engine called Andromeda (Cox et al., 2011) developed at the Max Planck Institute for Biochemistry. Besides protein and 
PTM identifications, MaxQuant is designed for a reliable quantification of stable isotopic labeled samples such as SILAC or dimethyl labeled samples based on 3D peak extraction at the MS1 level (Cox and Mann, 2008).

\subsubsection{Phosphosite identification and localization}

Phosphoprotein analysis by MS has similarities and differences compared to the general workflow of protein analysis. In this section, I will address the differences in more detail. The main challenge of the phosphosites identification by MS in DDA mode is that phosphorylation

occurs at sub-stoichiometric level. Therefore, enrichment is necessary for the phosphopeptides to be selected for fragmentation and subsequent identification. In section 1.4.1 several methods of phosphopeptides enrichment were introduced. The most common method is the complexing of phosphate group with metals, for instance, immobilized metal affinity chromatography (IMAC) (Villen and Gygi, 2008) or with metal oxides such as titanium/zirconium ( $\mathrm{TiO}_{2}$ or $\mathrm{ZrO}_{2}$ ) (Larsen et al., 2005). Furthermore, a highly specific phospho-tyrosine antibody has been developed to specifically enrich the phospho-tyrosine residues (Rush et al., 2005).

In the fragmentation step, depending on the type of fragmentation, the phosphopeptides are identified differently. CID fragmentation results in neutral loss of phosphoric acid $\left(\mathrm{H}_{3} \mathrm{PO}_{3}, 98\right.$ Da) by phosphorylated serines and threonines (Boersema et al., 2009a). Phospho-tyrosines produce a reporter immonium ions with the $\mathrm{m} / \mathrm{z}$ value of 216.043 (Steen et al., 2001). However, the low mass cut-off of ion trap makes the detection of immonium ions difficult (see section 1.4.1.3). In HCD fragmentation, the multiple cleavage events results in richer fragmentation of phosphopeptide (Jedrychowski et al., 2011). In contrast to CID and HCD, fragmentation by ETD do not result in the natural loss, leading to a higher quality of MS2 spectra (Quan L, 2013). Several studies have compared the phosphopeptide identification yield under different fragmentation methods. It was reported that HCD fragmentation is more efficient in the number of identified phosphosites and identification quality (higher Mascot score) than CID (Nagaraj et al., 2010) or ETD (Marx et al., 2013). Moreover, the unambiguous phosphosite localization in the phosphopeptides relies on the efficient fragmentation and the high-quality MS2 scan. Phosphosite localization is particularly challenging if several phosphorylable amino acids $(\mathrm{S}, \mathrm{T}, \mathrm{Y})$ happen to be present in a peptide close to each other. Therefore, each search engine and software calculates the localization 
score that determines the confidence of phosphorylation localization (for instance, localization probability in MaxQuant/Andromeda platform) (Sharma et al., 2014, Olsen et al., 2006b).

\subsubsection{Mass spectrometry-based quantification}

Mass spectrometers are able to measure two main parameters of the peptides: 1) the $\mathrm{m} / \mathrm{z}$ value of the precursor as well as the product ions and 2) their intensity. The $\mathrm{m} / \mathrm{z}$ values are used for the identification of the peptide/protein, whereas the intensities provide information about the abundance of peptides and can be used to collect the quantitative data. MS-based quantification methods can be categorized into two main groups: relative and absolute quantification. While in a relative quantification the abundance of the peptide/protein in one or more experimental treatments are compared to a control treatment, in an absolute quantification the concentration of proteins is determined (Table11). Also, the relative and absolute quantification methods can be subcategorized into two groups: label-free and label-based methods (Nikolov et al., 2012). The most common relative label-based method in the field is the Stable Isotope Labeling with Amino acids (SILAC) (Ong et al., 2002). Due to the incorporation of isotopic labels in the early stage of the experiment (at the protein level), this method is less prone to sample-handling errors and provides the most robust quantification of peptides/proteins. It has been shown that it is possible to label organisms such as mice by a diet containing heavy amino acids (Krüger et al., 2008). Here, we used a type of chemical labeling at the peptide level known as dimethyl labeling method.

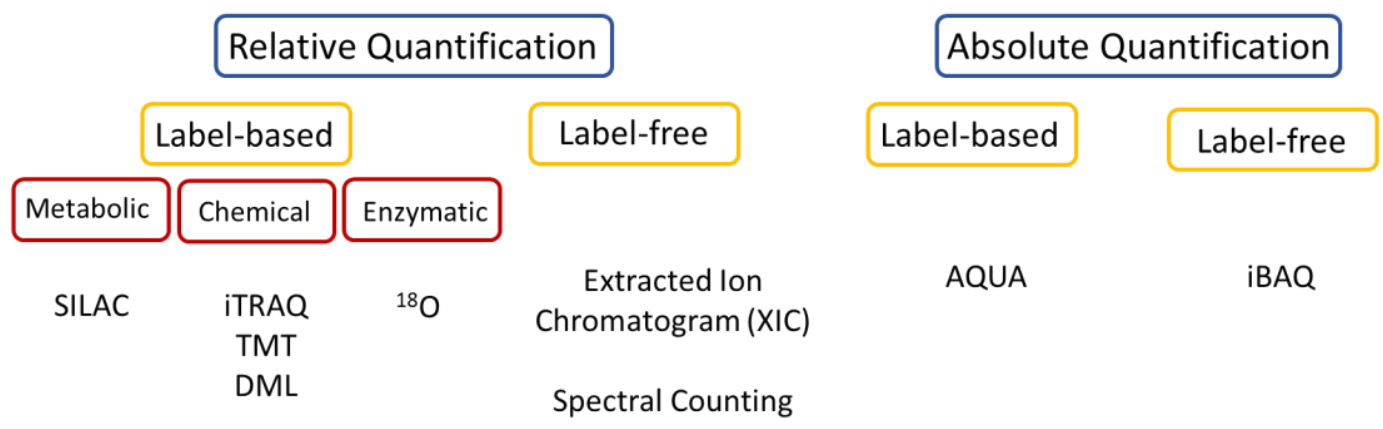

Table 1-1: Categorization of the most commonly used quantitative approaches in proteomics.

SILAC: Stable isotope labeling by amino acids in cell culture; iTRAQ: isobaric tags for relative and absolute quantification; TMT: tandem mass tag; DML: Dimethyl labeling; XIC: extracted ion chromatogram; AQUA: absolute quantitation; iBAQ: intensity based absolute quantitation, adapted from (Nikolov et al., 2012). 
Dimethyl labeling was introduced for the first time in 2003 as a method for a comparative quantification of proteome data (Hsu et al., 2003). In this approach, primary amines (Ntermini and lysine residues of side chains) are selectively dimethylated using formaldehyde and cyanoborohydride. The reaction involves formation of Schiff bases during the reaction of formaldehyde with the amine which then is reduced by cyanoborohydride. Using deuterium and ${ }^{13} \mathrm{C}$, it is possible to label three samples differentially (light, medium and heavy) (Figure 1-12). Dimethyl labeling has several advantages: The reaction is fast and is completed in a few minutes. It does not produce side products and it is compatible with the rest of downstream proteomics/phosphoproteomics workflow (Kovanich et al., 2012). Using deuterium in the labels leads to a small shift in the retention time of the labeled samples as compared to non-labeled samples and can affect quantification. However, if the quantification is based on the entire extracted ion peaks such as the algorithm that is designed in MaxQaunt, the deuterium effect on the quantification accuracy is decreased significantly and can be neglected (Ji and Li, 2005).

In recent years, the improvement in the instrumentation (high mass resolution, accuracy and high peptide identification rate) and computational power has made label-free quantification more popular as a quantification method.

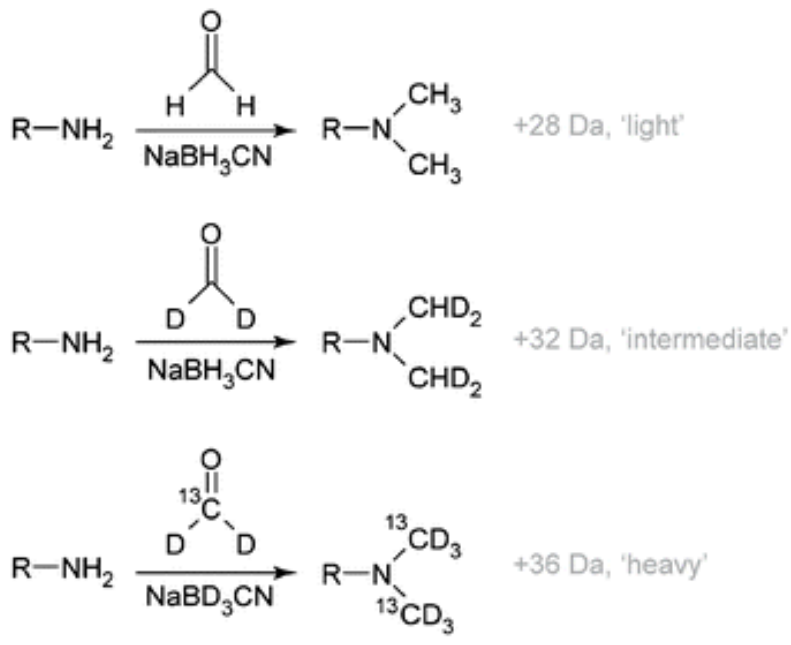

Figure 1-12: Labeling scheme of the reactions occurs in stable isotope dimethyl labeling. Using different isotopomers of the formaldehyde (Deuterium instead of hydrogen) and cyanoborohydride $\left({ }^{13} \mathrm{C}\right.$ instead of ${ }^{12} \mathrm{C}$ ), different masses with 4 Da difference can be added to the peptides which makes it possible to label three samples differently (light, medium, and heavy) and compare them, (Boersema et al., 2008). 
Two main approaches for label-free quantification have been introduced: 1) spectral counting that relies on the number of peptides which are identified from a given protein (Liu et al., 2004), and 2) comparing the peptide peak area in the extracted ion chromatogram (XIC) of different samples (Bondarenko et al., 2002). Recently, an improvement in quantification algorithm of intensity-based label-free quantification has been incorporated into the MaxQuant platform (MaxLFQ) which has made this method compatible with the upstream fractionation step. Also, it has increased the extraction of ratio information from peptide signals (Cox et al., 2014). Recently, label-free quantification has been gaining more popularity in the field due to its advantages over label-based methods such as improvement in the number of quantified peptides, possibility to compare an unlimited number of the samples and lower cost and effort. However, it has several drawbacks: 1) it is more prone to the sample handling variability, consequently less accurate quantitation, 2) it increases the MS measuring time and 3) it is less reliable for the quantification of modified peptides rather than proteins, since the quantitation information relies on quantification of one individual peptide (Cox et al., 2014, Mallick and Kuster, 2010).

\subsubsection{Neuro-phosphoproteomics}

In the last decade, phosphoproteomics has been used to answer diverse questions in the neuroscience field. In one of the earliest attempts, Ballif et al. tried to profile the phosphorylation sites of a developing mouse brain. Interestingly, they found phosphosites with the consensus site of the proline-directed kinase, phosphorylation sites upstream of acidic (such as CK2) or downstream of basic residues (for instance CaMKII) to be the most frequent phosphosites in their database (Ballif et al., 2004). In other studies, the phosphoproteome of developing and adult murine brain have been compared (Palmisano et al., 2012, Doubleday and Ballif, 2014) showing that neonatal brain contains three times more phosphosites than adult brain (Goswami et al., 2012). Moreover, using kinase knockout mice in combination with phosphoproteomics has revealed the role of kinases on the signaling pathways (e.g. cGMP-dependent protein kinase type I signaling pathway) (Corradini et al., 2014). Also, the impact of phosphorylation in neurodegenerative diseases such as Alzheimer has been addressed by MS-based phosphoproteomics (Tagawa et al., 2015). 
On the level of the synapse, studies can be divided into those addressing the post and presynaptic compartment. The majority of studies has focused on the postsynaptic phosphoproteome. Trinidad et al. in their studies mapped the postsynaptic phosphoproteome, which resulted in identification of an extensive number of phosphosites on the postsynaptic neurotransmitter receptors such as N-methyl-D-aspartate (NMDA) receptors and alpha-amino-3-hydroxy-5-methyl-4-isoxazolepropionic acid (AMPA) receptors. Also, they have shown that kinases and phosphatases are present in hippocampus at a higher level in comparison to the other parts of the brain, suggesting a major regulatory role for phosphorylation in hippocampus (Trinidad et al., 2006, Trinidad et al., 2008). In addition, in a study similar to the work presented here, quantitative analyses of phosphosites in resting and stimulated synapses have been performed. However, this study was focused on postsynaptic proteins (Munton et al., 2007). At the presynaptic level, Filiou et al. have provided a map of the phosphoproteome using isoelectric focusing (IEF) fractionation (Filiou et al., 2010). However, so far there is no high-throughput study available regarding the activity-dependent phosphorylation changes of the presynaptic proteins. 


\subsection{Aim of this study}

The presence of a high number of phosphoproteins, as well as kinases and phosphatases at the nerve terminals, suggests that reversible protein phosphorylation contributes to the regulation and fine-tuning of synaptic transmission. Previously, the role of phosphorylation in the regulation of neurotransmission in the nerve terminals has been studied by lowthroughput and targeted approaches or on the postsynaptic parts. Nevertheless, there is no comprehensive overview available regarding presynaptic regulation of neurotransmission via phosphorylation. Therefore, the major aim of this study was to investigate the activitydependent changes of presynaptic proteins phosphorylation by a quantitative and highthroughput approach. Tandem mass spectrometry-based phosphoproteomics is a suitable and unbiased approach to identify de novo phosphopeptides without any pre-knowledge. Moreover, in combination with labeling method, it is possible to relatively quantify the abundance of the phosphosites in the resting and stimulated conditions.

In this thesis, I have first established an MS-based phosphoproteomics workflow that enabled me to identify and reliably quantify the phosphosites in the nerve terminal. Next, I combined the established workflow with synaptosome stimulation treatments to distinguish between $\mathrm{Ca}^{2+}$-dependent and membrane depolarization-dependent phosphorylation changes. Using a bioinformatics approaches and kinase in vitro assays, I aimed to identify the upregulated and downregulated kinases/phosphatases responsible for the dynamics of phosphorylation changes upon stimulation.

Since $\mathrm{Ca}^{2+}$ ions are one of the main second messengers in the nerve terminal and their role in regulation of a high abundant kinase (CaMKII) and phosphatase (Calcineurin) in the nerve terminal is well-established, I used a specific inhibitors of CaMKII and Calcineurin (KN-93 and Cyclosporine A, respectively) in combination with the phosphoproteomics workflow to identify their substrates and also to assess the role of these enzyme in the signaling pathways triggered by stimulation of synaptosomes. Taken together, in this project, I aimed to provide a global picture of protein phosphorylation dynamics in the nerve terminal upon stimulation and elucidate the role of kinases and phosphatases during stimulation of neurotransmitter release. 


\section{Material and Methods}

\subsection{Materials}

\subsubsection{Chemicals}

The standard chemicals used in this study were purchased from Merck (Darmstadt, Germany) and Sigma-Aldrich (Steinheim, Germany). All chemicals had at least analytical purity. Other chemicals are listed in Table 2-1

\begin{tabular}{|l|l|}
\hline Chemicals & Source \\
\hline Albumin standard & Thermo Fisher Scientific, Rockford, USA \\
\hline Protease and phosphatase inhibitor & Thermo Fisher Scientific, Rockford, USA \\
\hline Acetonitrile & Thermo Fisher Scientific, Rockford, USA \\
\hline $\begin{array}{l}\text { 2-[4-(2-hydroxyethyl)piperazin-1- } \\
\text { yl]ethanesulfonic acid (Hepes) }\end{array}$ & Gerbu Biotechnik, Heidelberg, Germany \\
\hline Ethylene glycol tetraacetic acid (EGTA) & Gerbu Biotechnik, Heidelberg, Germany \\
\hline Formaldehyde (D2, 98\%, CD 20 ) & Cambridge Isotope Laboratories, Andover, USA \\
\hline Phenylmethylsulfonylfluorid(PMSF) & AppliChem, Darmstadt, Germany \\
\hline $\begin{array}{l}\text { 2-Amino-2-hydroxymethyl-propane-1,3-diol } \\
\text { (Tris) }\end{array}$ & VWR International, Leuven, Belgium \\
\hline Rapigest & Waters, Milford, USA \\
\hline Titanium dioxide beads (TiO2) & GL Sciences Inc., Tokyo, Japan \\
\hline Cyclosporine A & Sigma - Aldrich, Steinheim, Germany \\
\hline KN-93 & Sigma - Aldrich, Steinheim, Germany \\
\hline
\end{tabular}

Table 2-1: List of the chemicals used in this study. 


\subsubsection{Enzymes}

\begin{tabular}{|l|l|}
\hline Enzyme & Source \\
\hline Sequencing grade modified trypsin & Promega, Madison, USA \\
\hline Calmodulin-dependent Protein Kinase II (CaMKII) & New England BioLabs, Frankfurt, Germany \\
\hline Protein Kinase C (PKC) Enzyme System & Promega, , Meinheim, Germany \\
\hline Protein Kinase A (PKA) Enzyme System & Promega, , Meinheim, Germany \\
\hline
\end{tabular}

Table 2-2: Enzymes used in this study.

\subsubsection{Kits}

\begin{tabular}{|l|l|}
\hline Kits & Source \\
\hline $\begin{array}{l}\text { Bicinchoninic acid (BCA) protein } \\
\text { concentration assay }\end{array}$ & Thermo Fisher Scientific, Rockford, USA \\
\hline Western Lightening ${ }^{T M}$ Plus-ECL & $\begin{array}{l}\text { Perkin Elmer Applied Biosystems, Massachusetts, } \\
\text { USA }\end{array}$ \\
\hline
\end{tabular}

Table 2-3: Kits used in this study.

\subsubsection{Antibodies}

\begin{tabular}{|l|l|l|l|}
\hline Antibody & Species & $\begin{array}{l}\text { Application and } \\
\text { dilution }\end{array}$ & Source \\
\hline Synapsin 1 & $\begin{array}{l}\text { mouse monoclonal, affinity } \\
\text { purified }\end{array}$ & WB $(1: 1000)$ & $\begin{array}{l}\text { Synaptic } \\
\text { System }\end{array}$ \\
\hline pS603 Synapsin1 & rabbit polyclonal & WB $(1: 1000)$ & Rockland \\
\hline Dynamin $1 / 2 / 3$ & rabbit polyclonal, antiserum & WB $(1: 1000)$ & $\begin{array}{l}\text { Synaptic } \\
\text { System }\end{array}$ \\
\hline pS774 Dynamin & sheep polyclonal & WB $(1: 1000)$ & Rockland \\
\hline pS241PDK-1 & rabbit polyclonal & WB $(1: 1000)$ & Cell Signaling \\
\hline pT197 PKA C & rabbit polyclonal & WB $(1: 1000)$ & Cell Signaling \\
\hline pT514 PKC (Pan) & rabbit polyclonal & WB $(1: 1000)$ & Cell Signaling \\
\hline a-Tubulin & $\begin{array}{l}\text { mouse monoclonal, affinity } \\
\text { purified }\end{array}$ & WB $(1: 2000)$ & $\begin{array}{l}\text { Synaptic } \\
\text { System }\end{array}$ \\
\hline mouse IgG (HRP labeled ) & goat polyclonal & WB $(1: 2000)$ & BioRad \\
\hline rabbit IgG (HRP labeled ) & goat polyclonal & WB $(1: 2000)$ & BioRad \\
\hline sheep IgG (HRP labeled) & rabbit polyclonal & WB $(1: 2000)$ & Jackson \\
\hline
\end{tabular}

Table 2-4: Antibodies used in this study, WB (Western Blot). 


\subsubsection{Buffers}

\begin{tabular}{|c|c|}
\hline Buffers & Composition \\
\hline Homogenization buffer & $320 \mathrm{mM}$ sucrose, $5 \mathrm{mM}$ Hepes, $\mathrm{pH}=7.4$ \\
\hline Sodium buffer & $\begin{array}{l}10 \mathrm{mM} \text { glucose, } 5 \mathrm{mM} \mathrm{KCl}, 140 \mathrm{mM} \mathrm{NaCl}, 5 \mathrm{mM} \mathrm{NaHCO}_{3}, 1 \mathrm{mM} \\
\mathrm{MgCl}_{2}, 1.2 \mathrm{mM} \mathrm{Na}_{2} \mathrm{HPO}_{4}, 20 \mathrm{mM} \text { Hepes, } \mathrm{pH}=7.4\end{array}$ \\
\hline Lysis buffer & $\begin{array}{l}50 \mathrm{mM} \text { Tris, } 150 \mathrm{mM} \mathrm{NaCl}, 1 \% \text { Nonidet } \mathrm{P} 40, \mathrm{pH}=7.4 \text { contains } \\
\text { Pierce protease and phosphatase inhibitors }\end{array}$ \\
\hline Ficoll solutions & $\begin{array}{l}6 \%, 9 \% \text { and } 13 \% \text { Ficoll solutions were prepared in sucrose } \\
\text { buffer, } \mathrm{pH}=7.4\end{array}$ \\
\hline PMSF (Stock Solution) & $200 \mathrm{mM}$ stock solution in $100 \%$ ethanol \\
\hline Pepstatin A (Stock Solution) & $1 \mathrm{mg} / \mathrm{ml}$ \\
\hline TBST & 15 mM Tris-HCl, pH 7.4, 150 mM NaCl, 0.5 \% (v/v) Tween 20 \\
\hline Transfer buffer & 200 mM Glycine, 25 mM Tris, 0.04 \% SDS, 20 \% Ethanol \\
\hline SDS Running buffer & 25 mM Tris-HCl, 192 mM Glycine, $0.1 \%$ SDS \\
\hline
\end{tabular}

Table 2-5: List of buffers and their composition used in this study.

\subsection{Methods}

\subsubsection{Biochemical Methods}

\subsubsection{Synaptosomes preparation}

Synaptosomes were isolated from 5-6 weeks old Wistar rats as previously reported by Fischer and Mollard (Fischer von Mollard et al., 1991). See Figure 2-1 for a schematic representation of our synaptosomes preparation procedure. Briefly, two rats were decapitated and cortices and cerebellum were dissected. Samples were homogenized using a glass-Teflon homogenizer (RW20-DZM, IKA) in $30 \mathrm{ml}$ ice-cold homogenization buffer (containing $200 \mu \mathrm{M}$ PMSF and Pepstatin A $1 \mu \mathrm{g} / \mathrm{ml}$ ) at $900 \mathrm{rpm}, 9$ strokes. The homogenate was centrifuged for $2 \mathrm{~min}$ at $5000 \mathrm{rpm}, 4^{\circ} \mathrm{C}$ in a SS34 rotor (SORVALL) to remove cell debris. The supernatant was collected in a new tube and re-centrifuged for $12 \mathrm{~min}, 11000 \mathrm{rpm}, 4^{\circ} \mathrm{C}$ in the SS34 rotor. The supernatant containing the brain cytosol fraction was discarded, and the white pellet containing synaptosomes was resuspended in $6 \mathrm{ml}$ of homogenization buffer. At this step, the brown pellet, mostly containing mitochondria was cautiously avoided to be resuspended. Three $\mathrm{ml}$ of the suspension were loaded on a discontinuous Ficoll gradient (one 
gradient per cortex) consisting of 13\% Ficoll (4 ml), 9\% Ficoll (1 ml) and 6\% Ficoll (4 ml). Gradients were centrifuged for $35 \mathrm{~min}$ in a SW41 swing-out rotor (Beckman) at $22500 \mathrm{rpm}$. The band at the interface between 9\% and 13\% Ficoll was collected, transferred into a SS34 tube, diluted with $30 \mathrm{ml}$ of homogenization buffer, and re-centrifuged for $12 \mathrm{~min}$ at 11000 $\mathrm{rpm}\left(4{ }^{\circ} \mathrm{C}\right)$ to pellet synaptosomes. Finally, synaptosomes were resuspended in $5 \mathrm{ml}$ of homogenization buffer ( $2.5 \mathrm{ml}$ per cortex), and the protein concentration was determined using the BCA reaction kit. Normally, the yield was between 5-6 mg of synaptosomal proteins per cortex (Figure 2-1)

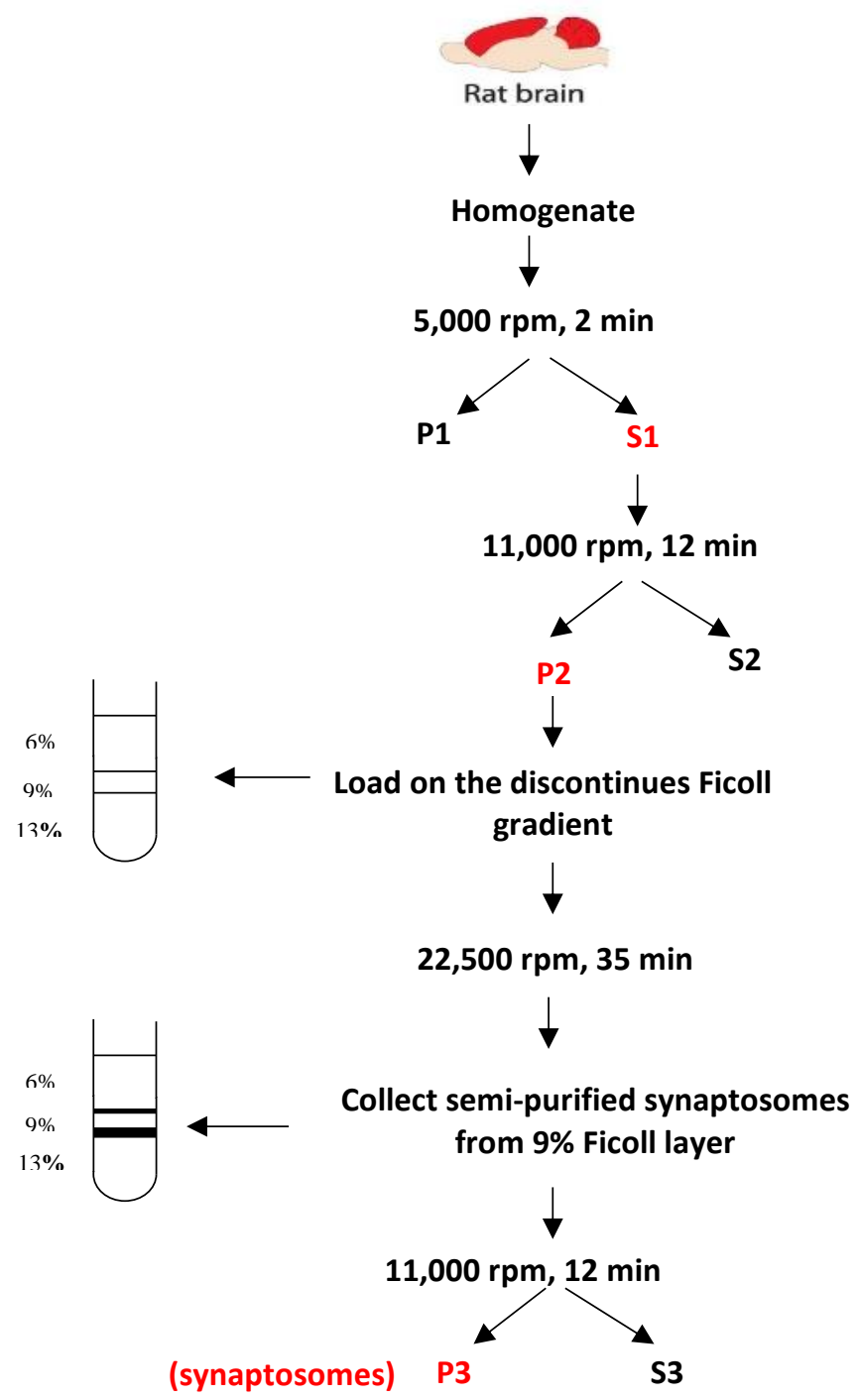

Figure 2-1: The flow diagram of purification of synaptosome from rat brains. 


\subsubsection{Protein concentration assay (BCA)}

To determine the protein concentration, the Pierce BCA Proteins Assay Kit (Thermo Fisher) was used. Standard solutions were prepared from Albumin (BSA) in the desired buffer and protein concentrations were measured in a 96-well plate using a Tecan Genios Pro plate reader according to manufacturer's manual.

\subsubsection{Synaptosomes stimulation (Glutamate Release Assay)}

\section{KCl stimulation}

For stimulation, $2.5 \mathrm{mg}$ of synaptosomal proteins were centrifuged for $5 \mathrm{~min}, 5000 \mathrm{~g}$, RT. Obtained pellet was resuspended very gently in $2.5 \mathrm{ml}$ of sodium buffer. Synaptosomes were then incubated for $5 \mathrm{~min}$ at $37^{\circ} \mathrm{C}$ ina water bath. In the next step, $\mathrm{NADP}^{+}(1 \mathrm{mM})$ and either $\mathrm{CaCl}_{2}(1.3 \mathrm{mM})$ or EGTA $(0.5 \mathrm{mM})$ were added depending on the stimulation condition. Samples were immediately transferred to a quartz cuvette with a stirrer bar and fluorescence was measured at $340 \mathrm{~nm}$ (excitation) and $440 \mathrm{~nm}$ (emission). After three min glutamate dehydrogenase (200 U) was added and measurements were continued for another 3 min. Subsequently, $\mathrm{KCl}$ (50 mM) was added to the reaction to stimulate synaptosomes. Glutamate dehydrogenase couples the conversion of glutamate to $\alpha$-ketoglutarate to NADP+ to NADPH reduction (Figure 2-2). The release of glutamate was monitored by fluorescence measurement of NADPH for 2 min. For the synaptosomes at the resting condition only NADP+, EGTA, and glutamate dehydrogenase were added (No $\mathrm{KCl}$ addition). For the inhibition of CaMKII and Calcineurin, synaptosomes were incubated prior to resuspension in sodium buffer for 10 min with $10 \mu \mathrm{M}$ KN-93 and $10 \mu \mathrm{M}$ Cyclosporine A, respectively.

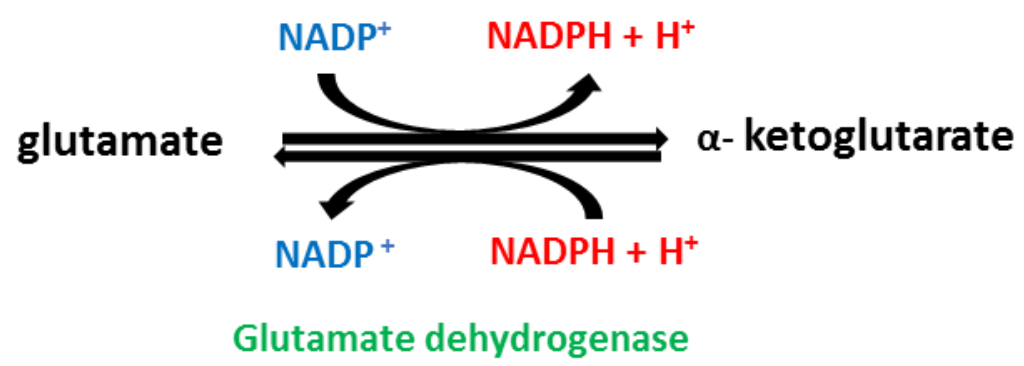

Figure 2-2: Conversion of glutamate to $\alpha$-ketoglutarate is coupled to the reduction of NADP+ to NADPH by Glutamate dehydrogenase. 


\section{Electrical stimulation}

For electrical stimulation, all the steps were done similar to $\mathrm{KCl}$ stimulation except application of $\mathrm{KCl}$. Instead of $\mathrm{KCl}$, synaptosomes were subjected to electrical stimulation through the platinum wire with $1 \mathrm{~mm}$ diameter. The diameter of the cuvette and the distance between the electrodes were $15 \mathrm{~mm}$. Stimulation was done by square pulses for alternating polarity with a frequency of $20 \mathrm{~Hz}$, and a voltage of $7 \mathrm{~V}$.

\subsubsection{Sample collection for mass spectrometry}

Stimulated synaptosomes were collected directly from the cuvette following glutamate release assay and were centrifuged for $2 \mathrm{~min}$ at $6200 \mathrm{~g}, 4^{\circ} \mathrm{C}$. The synaptosome pellet was resuspended in $100 \mu \mathrm{l}$ of lysis buffer containing mixture of protease and phosphatase inhibitors (Thermo Fisher Scientific) and incubated on ice for $30 \mathrm{~min}$. Then $900 \mu \mathrm{l}$ of $100 \mathrm{mM}$ TEAB buffer were added, and the synaptosomes were homogenized (RW20-DZM, IKA) with 3 strokes at maximum speed (2000 rpm). The protein concentration was determined by BCA protein concentration assay. $1 \mathrm{mg}$ of protein per sample was precipitated by $100 \%$ acetone overnight at $-20^{\circ} \mathrm{C}$. The next day, proteins were spun down for $30 \mathrm{~min}$ at $16200 \mathrm{~g}, 4^{\circ} \mathrm{C}$. The pellets were washed once with $80 \%$ acetone and centrifuged again for $30 \mathrm{~min}$ at $16200 \mathrm{~g}, 4$ ${ }^{\circ} \mathrm{C}$. The protein pellet was dried shortly at RT and later stored at $-20{ }^{\circ} \mathrm{C}$ for the next step of the protocol (protein digestion).

\subsubsection{SDS-PAGE and Western Blotting}

For the analysis of the proteins, $10 \mu \mathrm{g}$ of samples were mixed with NuPAGE lithium dodecyl sulfate (LDS) sample buffer (Thermo Fisher Scientific) and loaded on a RunBlue SDS gradient gel (Expedeon), a pre-casted 4-12\% polyacrylamide gel. Electrophoresis was performed in Tris-MOPS-SDS run buffer (Expedeon) for 60-90 min at 100-120 V.

Western blotting was done according to a previously published protocol (Liu et al., 2014). Separated proteins were transferred to a nitrocellulose membrane in transfer buffer using a tank apparatus and under $100 \mathrm{~V}$ constant voltage application for $1 \mathrm{~h}$. After transfer, nitrocellulose membrane was blocked by $5 \%$ nonfat milk powder in TBST for $30 \mathrm{~min}$, RT. Then, the membrane was incubated overnight at $4{ }^{\circ} \mathrm{C}$ with primary antibodies diluted in 
blocking buffer. Membrane was washed 3 times with TBST for $5 \mathrm{~min}$ and then incubated with HRP-conjugated secondary antibodies in blocking buffer for 1 hour at room temperature. The membrane was again washed 3 times with TBST for 5 min and then covered with Western Lightning Plus-ECL oxidizing reagent and enhanced luminol reagent (PerkinElmer) mixed in 1:1 ratio and visualized by using chemiluminescence detection on a LumiImager (Boehringer Ingelheim).

\subsubsection{In vitro kinase assays for mapping of the kinases responsible for the phosphorylation of specific sites}

In vitro kinase assays were carried out using the ADP-Glo ${ }^{\mathrm{TM}}$ kinase assay kit (Promega). This assay is a luminescent kinase assay that measures ADP that is generated from a kinase reaction. ADP is converted to ATP and ATP is converted into light by luciferase. The generated luminescent signal is proportional to the amount of kinase activity. First, standard curves were obtained by measuring the luminescence a series of ADP-ATP dilutions, based on the manufacturer's manual. For each enzyme, the concentration of ATP and kinase were optimized to achieve $100 \%$ conversion of ATP to ADP using the positive control peptides. In all assays $0.2 \mu \mathrm{g} / \mu \mathrm{l}$ of each peptide were used. Then, phosphorylation of desired phosphosites by the respective kinase was calculated by measuring the luminescence of the reaction, which is proportional to ATP consumption by the kinase. The percentage of consumed ATP was used to characterize the $\%$ of the kinase activity. CaMKII was activated prior to the assay by addition of $5 \mu \mathrm{M} \mathrm{ATP}, 1.2 \mu \mathrm{M}$ calmodulin and $2 \mathrm{mM} \mathrm{CaCl}_{2}$, followed by 30 min incubation at $37^{\circ} \mathrm{C}$. In Table 2-6 the concentration of the reagents that were used in each kinase assay are listed. The peptides are purchased from Thermo Scientific with a purity of $98 \%$. Table 2-7 provides the list and the sequences of the peptides that were used in the assay.

\begin{tabular}{|l|l|l|}
\hline Kinase (concentration) & Positive control peptide (Source) & ATP concentration \\
\hline CaMKII $(12 \mathrm{U} / \mu \mathrm{l})$ & Autocamide (GenScript) & $5 \mu \mathrm{M}$ \\
\hline PKA $(0.7 \mathrm{U} / \mu \mathrm{l})$ & Kemptide (Promega) & $5 \mu \mathrm{M}$ \\
\hline PKC $(5 \mathrm{ng} / \mu \mathrm{l})$ & Neurogranin (Promega) & $25 \mu \mathrm{M}$ \\
\hline
\end{tabular}

Table 2-6: Enzymes, control peptides and chemicals used in in vitro kinase assays. 


\begin{tabular}{|l|l|l|l|}
\hline \multicolumn{1}{|c|}{ Sequence } & \multicolumn{1}{|c|}{ Name } & \multicolumn{1}{c|}{ Protein ID } & \multicolumn{1}{c|}{ Length (aa) } \\
\hline SLQRQLSIALPQE & Liprin-a3_S75 & F1LSE6 & 13 \\
\hline SLQRQLAIALPQE & Liprin-a3_S75 & F1LSE6 & 13 \\
\hline MMQRSMSDPKPLS & Pclo_S3626 & Q9JKS6-2 & 13 \\
\hline MMQRSMADPKPLS & Pclo_A3626 & Q9JKS6-2 & 13 \\
\hline TLQRSLSDPKPLS & Bsn_S2844 & G3V984 & 13 \\
\hline TLQRSLADPKPLS & Bsn_A2844 & G3V984 & 13 \\
\hline VPVRSGSIEQASL & Rim1_S1141 & F1LYS1 & 13 \\
\hline VPVRSGAIEQASL & Rim1_A1141 & F1LYS1 & 13 \\
\hline RHERRHSDVALPH & Rim1_S413 & F1LYS1 & 13 \\
\hline RHERRHADVALPH & Rim1_S413 & F1LYS1 & 13 \\
\hline GPIRQASQAGPGP & Syn1_S603 & P09951 & 13 \\
\hline
\end{tabular}

Table 2-7: Sequence of peptides used in in vitro kinase assay.

\subsubsection{Mass spectrometry methods}

\subsubsection{Protein digestion}

For protein digestion, the dried protein pellets were dissolved in $80 \mu \mathrm{l}$ of $1 \%$ Rapigest by vortexing, sonication and incubation in the thermoshaker at $60{ }^{\circ} \mathrm{C}$ for $15 \mathrm{~min}$ at $1050 \mathrm{rpm}$. Dissolved protein were reduced by addition of $20 \mu \mathrm{l}$ of $50 \mathrm{mM}$ DTT and incubation at $60{ }^{\circ} \mathrm{C}$ for $45 \mathrm{~min}$ at $1050 \mathrm{rpm}$. Following, proteins were alkylated by addition of $20 \mu \mathrm{l}$ of $100 \mathrm{mM}$ CAA and incubation at $37^{\circ} \mathrm{C}$ for $45 \mathrm{~min}$ at $750 \mathrm{rpm}$. Then the concentration of RapiGest in the samples were reduced to $0.1 \%$ by addition of $100 \mathrm{mM} \mathrm{TEAB}(\mathrm{pH}=8)$. Then the proteins were digested by adding trypsin in 1:20 ratio (trypsin to protein ratio) and incubation overnight in the thermoshaker at $37^{\circ} \mathrm{C}$ and $750 \mathrm{rpm}$. To stop the digestion reaction and degradation of RapiGest, 1\% FA was added to the samples and incubated at $37{ }^{\circ} \mathrm{C}$, for $2 \mathrm{~h}$, at $750 \mathrm{rpm}$. RapiGest precipitations were cleared by centrifugation at $13000 \mathrm{rpm}$ for $30 \mathrm{~min}$ at $4{ }^{\circ} \mathrm{C}$. The supernatant containing the peptides were transferred to a new tube and dried in vacuum 
concentrator and kept in $-20{ }^{\circ} \mathrm{C}$ for the next step (Dimethyl labeling). All the reagents were dissolved in $100 \mathrm{mM}$ TEAB, $\mathrm{pH}=8$.

\subsubsection{Stable isotope dimethyl labeling}

Digested samples were differentially labeled by stable isotope dimethyl labeling according to Boersema et al. (Boersema et al., 2009b). Briefly, the digested samples were dissolved in 100 $\mu \mathrm{l}$ of TEAB (100 mM, pH=8) and separately labeled by light and medium isotope tags by adding $4 \mu \mathrm{l}$ of $4 \%$ (vol/vol) $\mathrm{CH}_{2} \mathrm{O}$ and $4 \mu \mathrm{l}$ of $4 \%$ (vol/vol) $\mathrm{CD}_{2} \mathrm{O}$ to the light and medium samples, respectively. Then $4 \mu \mathrm{l}$ of $0.6 \mathrm{M} \mathrm{NaBD}_{3} \mathrm{CN}$ was added to both light and medium samples, and samples were incubated at RT for $1 \mathrm{~h}$ at $750 \mathrm{rpm}$ in the thermoshaker. To stop the reaction, $16 \mu \mathrm{l}$ of $1 \%$ (vol/vol) ammonia and $8 \mu \mathrm{l}$ of 5\% FA (vol/vol) were added to the samples. Then the light and medium samples were mixed to a volume ratio of 1:1 and kept on ice for the fractionation by strong cation exchange.

\subsubsection{Strong cationic exchange (SCX) chromatography}

The fractionation of the peptides by SCX chromatography was performed on a FPLC system (SMART, Pharmacia). The mixed light/medium peptides were diluted with the solvent A (10 $\left.\mathrm{mM} \mathrm{NH}_{4} \mathrm{HCO}_{2}, 30 \% \mathrm{ACN}(\mathrm{v} / \mathrm{v}), \mathrm{pH}=2.7\right)$ to a final volume of $1 \mathrm{ml}$ and then loaded on a Mono S column PC 1.6/5 (Pharmacia Biotech). The peptides were eluted with a gradient of 0-90\% solvent $\mathrm{B}\left(500 \mathrm{mM} \mathrm{NH} 4 \mathrm{HCO}_{2}, 30 \%\right.$ ACN (v/v), $\left.\mathrm{pH}=2.7\right)$ over $40 \mathrm{~min}$ at a flow rate of 100 $\mu \mathrm{l} / \mathrm{min}$. The first twelve fractions, $0.2 \mathrm{ml}$ each, including the flow-through were collected and dried in the vacuum concentrator and were subjected to the phosphopeptides enrichment separately.

\subsubsection{Phosphopeptide Enrichment}

\section{$\mathrm{TiO}_{2}$ enrichment}

The spin column phosphopeptide enrichment by $\mathrm{TiO} 2$ was performed mainly according to Larson et al (Larsen et al., 2005) with some modifications. In-house $\mathrm{TiO}_{2}$ columns were packed with the $\mathrm{TiO}_{2}$ beads (GL Sciences Inc., Tokyo, Japan) and equilibrated with loading buffer $(200 \mathrm{mg} / \mathrm{ml}$ DHB in 80\% ACN, 5\% TFA). Dried peptides were dissolved in $60 \mu \mathrm{l}$ of loading buffer and loaded onto a $\mathrm{TiO}_{2}$ column by centrifugation at $3000 \mathrm{rpm}$ for $5 \mathrm{~min}$ at RT (all the washing and elution steps were done with the same settings). The columns were 
washed three times with $60 \mu \mathrm{l}$ of loading buffer and five times with $60 \mu \mathrm{l}$ washing buffer ( $80 \%$ ACN, $5 \%$ TFA). Then, phosphopeptides were eluted by $40 \mu \mathrm{l}$ of $0.3 \mathrm{~N} \mathrm{NH}_{4} \mathrm{OH}, \mathrm{pH} \geq 10.5$ (three times). Eluted phosphopeptides were dried in the vacuum concentrator for further MS analysis.

\section{Immobilized metal affinity chromatography (IMAC) enrichment}

IMAC enrichment was performed based on a previously described protocol (Villén and Gygi, 2008). Briefly, $100 \mu \mathrm{l}$ of IMAC beads (PhosSelect iron affinity gel, Sigma) were washed three times with $1 \mathrm{ml}$ of binding buffer ( $40 \% \mathrm{ACN}$ ( $\mathrm{vol} / \mathrm{vol}$ ), $25 \mathrm{mM} \mathrm{FA}$ in $\mathrm{H}_{2} \mathrm{O}$ ) and $50 \%$ slurry of the beads were prepared. Each peptide fraction from SCX chromatography was dissolved in $120 \mu \mathrm{l}$ binding buffer, and $10 \mu \mathrm{l}$ of IMAC beads was added. Peptides were incubated with the beads at RT for $1 \mathrm{~h}$ with vigorous shaking. Then resins were washed three times with $120 \mu \mathrm{l}$ of IMAC binding buffer. The phosphopeptides were then eluted three times by incubation of the resin with elution buffer ( $\left.50 \mathrm{mM} \mathrm{K} \mathrm{HPO}_{4} / \mathrm{NH}_{4} \mathrm{OH}, \mathrm{pH} 10.0\right)$ for 5 min. In order to neutralize the eluted solution, $40 \mu \mathrm{l}$ of $10 \%$ FA was added to the tubes. Eluted phosphopeptides were dried in the vacuum concentrator for further MS analysis.

\subsubsection{LC-MS/MS analysis}

For the analysis of the phosphopeptides in this study, three mass spectrometers were used. For the optimization part the LTQ Orbitrap Velos (section 3.1), for the stimulation of synaptosomes in different conditions the $Q$ Exactive (section 3.2) and for the kinase/phosphatase inhibition experiment the Q Exactive HF (section 3.3) were used. In the following paragraphs, the operating parameters of each instrument are described.

\section{LTQ Orbitrap Velos}

Peptides were analyzed on hybrid linear ion trap-Orbitrap Velos (Thermo Scientific) mass spectrometer coupled to a nanoflow liquid chromatography system (Agilent 1100 series, Agilent). The peptides were pre-concentrated on a trap column $(100 \mu \mathrm{m}$ ID $\times 20 \mathrm{~mm}$ selfpacked with Reprosil-Pur 120 C18-AQ, 5 mm, Dr. Maisch GmbH, Ammerbuch-Entringen, Germany) at $5 \mu \mathrm{l} / \mathrm{min}$ in loading buffer ( $2 \% \mathrm{ACN}, 0.1 \% \mathrm{FA})$ and then separated by an analytical column (75 $\mu \mathrm{m}$ ID $\times 200$ mm self-packed with Reprosil-Pur 120 C18-AQ, $3 \mu \mathrm{m}$, Dr. Maisch $\mathrm{GmbH}$ ) using a linear gradient form 5\% to 34\% buffer B (95\% ACN and $0.1 \%$ FA) at flow rate of $300 \mathrm{nl} / \mathrm{min}$. The mass spectrometer was operated in data-dependent acquisition 
mode. Full scan MS spectra were acquired in the range of $350-1600 \mathrm{~m} / \mathrm{z}$ at a resolution of $30,000 \mathrm{FWHM}$ at $\mathrm{m} / \mathrm{z} 400$. The top 10 most intense peaks per cycle from the survey scan were selected for fragmentation in the linear ion trap with Collision Induced Dissociation (CID) with the normalized collision energy (NCE) of 35\% and isolation window $2 \mathrm{~m} / \mathrm{z}$. Dynamic exclusion time for precursor ions selected for MS/MS fragmentation was $60 \mathrm{~s}$. Automatic gain control target value and maximum ion injection time for MS and MS/MS were $1 \times 10^{6}$ in $500 \mathrm{~ms}$ and $1 \times 10^{4}$ in $100 \mathrm{~ms}$, respectively.

\section{Q-Exactive}

Enriched phosphopeptides in each fraction were analyzed on a Q-Exactive hybrid Quadrupole-Orbitrap mass spectrometer (Thermo Scientific) coupled to a NanoLC pump (EASY-nLC 1000, Thermo Scientific). The peptides were pre-concentrated on a Reversed Phase-C18 precolumn (100 $\mu \mathrm{m}$ ID × 20 mm self-packed with Reprosil-Pur 120 C18-AQ $5 \mu \mathrm{m}$, Dr. Maisch GmbH, Ammerbuch-Entringen, Germany) at flow rate $5 \mu \mathrm{l} / \mathrm{min}$ in the loading buffer $(2 \% \mathrm{ACN}, 0.1 \% \mathrm{FA})$ and then separated by reversed phase-C18 nanoflow chromatography $(75 \mu \mathrm{m}$ ID $\times 200$ mm self-packed with Reprosil-Pur 120 C18-AQ, $3 \mu \mathrm{m}$, Dr. Maisch $\mathrm{GmbH})$. Peptides were injected with solvent A (0.1\% FA) at a flow rate $320 \mathrm{~nL} / \mathrm{min}$ and eluted by $0-37 \%$ solvent B (95\% ACN, $0.1 \% \mathrm{FA})$ with an overall run-time of $45 \mathrm{~min}$. Separated peptides were ionized by electrospray ionization (ESI) source in a positive ion mode. The mass spectrometer was operated in data-dependent acquisition mode. Full scan MS spectra were acquired in the range of 350-1600 m/z at a resolution of of 70,000, m/z 200. The top 12 most intense peaks per cycle from the survey scan were selected for fragmentation in the collision cell with Higher-energy Collisional Dissociation (HCD) with the normalized collision energy (NCE) of 28\% and isolation window $2 \mathrm{~m} / \mathrm{z}$. Dynamic exclusion time for precursor ions selected for MS/MS fragmentation was $20 \mathrm{~s}$. Automatic gain control target value and maximum ion injection time for MS and MS/MS were $1 \times 10^{6}$ in $60 \mathrm{~ms}$ and $1 \times 10^{5}$ in $120 \mathrm{~ms}$, respectively.

\section{Q-Exactive HF}

Enriched phosphopeptides in each fraction were dissolved in loading buffer ( $2 \%$ ACN, $0.1 \%$ FA) and analyzed on a Q-Exactive HF hybrid Quadrupole-Orbitrap mass spectrometer (Thermo Scientific) coupled to an Ultimate 3000 RSLC UHPLC (Dionex, USA). The peptides 
were pre-concentrated on a Reversed Phase-C18 precolumn (100 $\mu \mathrm{m}$ ID $\times 28 \mathrm{~mm}$ self-packed with Reprosil-Pur 120 C18-AQ $1.9 \mu \mathrm{m}$, Dr. Maisch GmbH, Ammerbuch-Entringen, Germany) and then separated by reversed phase-C18 nanoflow chromatography $(75 \mu \mathrm{m}$ ID $\times 200 \mathrm{~mm}$ self-packed with Reprosil-Pur 120 C18-AQ, $3 \mu \mathrm{m}$, Dr. Maisch GmbH). Peptides were injected with solvent $\mathrm{A}(0.1 \% \mathrm{FA})$ at a flow rate $300 \mathrm{~nL} / \mathrm{min}$ and eluted by $5-46 \%$ solvent $\mathrm{B}(80 \%$ ACN $0.08 \% \mathrm{FA}$ ) with an overall run-time of $88 \mathrm{~min}$. Separated peptides were ionized by electrospray ionization (ESI) source in a positive ion mode. The mass spectrometer was operated in data-dependent acquisition mode. Full scan MS spectra were acquired in the range of $350-1600 \mathrm{~m} / \mathrm{z}$ at a resolution of at a resolution of 120,000 . The top 15 most intense peaks per cycle from the survey scan were selected for fragmentation in the collision cell with Higher-energy Collisional Dissociation (HCD) with the normalized collision energy (NCE) of $28 \%$ and isolation window $2 \mathrm{~m} / \mathrm{z}$. Dynamic exclusion time for precursor ions selected for MS/MS fragmentation was $30 \mathrm{~s}$. Automatic gain control target value and maximum ion injection time for MS and MS/MS were $1 \times 10^{6}$ in $50 \mathrm{~ms}$ and $1 \times 10^{5}$ in $110 \mathrm{~ms}$, respectively.

\subsubsection{MS raw file processing}

The output file from LC-MS/MS runs contain the retention time of the peptides, $\mathrm{m} / \mathrm{z}$ value, intensities as well as the precursor ions and product ions of selected peptides. For the identification and quantification of phosphopeptides the acquired raw files were processed using the MaxQuant software package version 1.5.0.25 (Cox and Mann, 2008). Spectra were searched using the Andromeda search engine (Cox et al., 2011) against the proteome database of Rattus Norvegicus (Uniprot complete proteome updated at 2015-01-07, with 29,378 entries). MaxQuant search was configured as follows; For the MS1 first and the main search the mass tolerance was set to 20 and $4.5 \mathrm{ppm}$, respectively. The multiplicity was set to two (DimethLys0 and DimethNter0 was for 'light' and DimethLys4 and DimethNter4 for 'heavy' samples). Trypsin/P was fixed as protease and 7 amino acids as the minimum peptide length, and maximum of two missed cleavages were allowed. Carbamidomethylation of cysteine was set as fixed modification and methionine oxidation, acetylation (on $\mathrm{N}$-terminal), phosphorylation on serine/threonine/tyrosine were specified as variable modifications. False discovery rate of $1 \%$ was applied and re-quantification was enabled. For the optimization part, to search for the unlabeled peptides, the multiplicity was set to one and 
the phosphorylation of serine/threonine/tyrosine phosphorylation was not set as variable modification.

\subsubsection{Data processing and bioinformatics analysis}

For the downstream data analysis, the resulting phospho(STY).txt output file from the MaxQuant was processed by Perseus software version 1.5.0.9 (Cox and Mann, 2008). Perseus data processing was configured as follows; only the phosphosites quantified in at least 2 biological replicates were selected for further analysis. Also the reverse hits of phosphosites were filtered out from data processing. Moreover, only Class I phosphosites (phosphosites with localization probability more than 0.75 ) (Olsen et al., 2006a) were accepted for further analysis. The mean (in synaptosomes stimulation in different conditions experiments) or median (in Calcineurin and CaMKII inhibition experiments) of normalized ratios and the intensities of phosphosites across the biological replicates were extracted and transformed to $\log 2$ and $\log 10$, respectively. The transformed values were used to plot the distribution of quantified phosphosites (Figure 3-10 A, B, C, Figure 3-19 A and Figure 3-23 A).

The significantly regulated phosphosites were determined for each experiment using Perseus software as follows: For experiment including stimulation of synaptosomes in different conditions (section 3.2), significant outliers were determined by two-tailed 'Significant A' test with the $p$-value $\leq 0.05$. For the Calcineurin inhibition experiments (section 3.2.1), significant outliers were determined by two-tailed 'Significant B' test with the $p$-value $\leq 0.05$. 'Significant B' test determines the outliers in an intensity-dependent manner, whereas the 'Significant A' test only take the log2 ratios as the input (Cox and Mann, 2008, Cox et al., 2009). For the CaMKII inhibition experiments, two layers of regulated phosphosites were defined due to the shift towards the downregulated phosphosites upon CaMKII inhibition (see Figure 3-23 A): First, the outliers by one-tailed 'Significant B' test with the $p$-value $\leq 0.05$. Second, all the phosphosites that are downregulated by at least 2 folds upon stimulation (log2 ratio $=1)$.

\subsubsection{Motif-X analysis}

To determine the enriched motifs in up and downregulated phosphosites, Motif-X tool (Schwartz and Gygi, 2005) was used remotely from motif-x.med.harvard.edu/motif-x.html. Sequences of upregulated or downregulated phosphorylation sites in the comparisons were 
independently searched against the IPI Rat Proteome as background. Central character was set as " $\mathrm{S}$ " and sequence length was specified as 31. For experiment including stimulation of synaptosomes in different conditions (section 3.2), the default suggested setting were used for the analysis. For the Calcineurin and CaMKII inhibition experiments (section 3.2.1 and 3.2.2), the occurrences and significance parameters were set to 10 and 0.00001 , respectively.

\subsubsection{Other tools, software and databases}

For the illustration of proteins, domains and phosphosites in Figures 3-13, 3-14 B and 3-15 the DOG software (Ren et al., 2009) was used. The area-proportional venn diagram plotter of BxToolBox system was used to draw the venn diagrams. The PhosphoSitePlus database was used to report the identified phosphosites in previous studies. 


\section{Results}

\subsection{Establishment of a protocol for the identification and quantification of synaptosomal phosphopeptides}

As it was mentioned in introduction, several phosphopeptides enrichment protocols have been described. Here, I have optimized parameters in the different steps of sample preparation workflow such as digestion, enrichment, and peptide labeling to establish a protocol for identification and reliable quantification of the synaptosomal phosphosites. The most important parameters that I have optimized are the following:

i. Protein digestion (amount of starting material and protein acetone precipitation).

ii. Titanium dioxide $\left(\mathrm{TiO}_{2}\right)$ enrichment of phosphopeptides.

iii. Peptides fractionation by strong cation exchange chromatography (SCX)

iv. Immobilized metal affinity chromatography (IMAC) as the alternative enrichment method.

\subsubsection{Protein digestion and phosphopeptides enrichment by $\mathrm{TiO}_{2}$}

Following isolation of synaptosomes according to the protocol that was described in section 2.2.1.1, different amounts of synaptosomes ( $25 \mu \mathrm{g}$ to $1 \mathrm{mg}$ ) were directly solubilized in $10 \mu \mathrm{l}$ of 1\% (W/V) RapiGest and were digested by trypsin with a protein to enzyme ratio of 50:1. RapiGest is a commercial surfactant that solubilizes proteins and facilitates the enzymatic digestion. The advantage of the RapiGest over the commonly used denaturant such as SDS or urea is that it does not modify the peptides structures and does not suppress the tryptic enzyme activity. It undergoes hydrolysis in acidic conditions, and one of the fragment precipitates, so it can be removed from the solution by a centrifugation step, whereas the second hydrolytic fragment is water soluble and is unretained on the reverse phase column. This makes it compatible with the MS workflow. Here, it was observed that by increasing the amount of synaptosomes for the digestion, the number of identified peptides was decreasing (Figure 3-1 A). This might be due to the incomplete solubilization of proteins upon increase of the protein:RapiGest ratio. However, the number of identified peptides was improved significantly by acetone precipitation of the $1 \mathrm{mg}$ of synaptosomal proteins prior to the 
solubilization with the RapiGest (Figure 3-1 A). Therefore, I have applied the acetone precipitation in all the following experiments.

The next step was to enrich the phosphosites from the pool of non-phosphorylated peptides generated by trypsinization. For this purpose, we have used the $\mathrm{TiO}_{2}$ enrichment method. In this method phosphopeptides with the negative charge will bind to the $\mathrm{TiO}_{2}$ resins in the acidic environment and will be eluted in the basic conditions. The specificity of the phosphopeptides binding was improved by applying the multifunctional acids such as the 2,5-dihydroxybenzoic acid (DHB) (Larsen et al., 2005). One of the common ways to use the $\mathrm{TiO}_{2}$ resins is to pack them in the pipet tips. Here, we have tested the different amounts of $\mathrm{TiO}_{2}$ resins in two different sizes of the pipet tips, to optimize the overall size of the $\mathrm{TiO}_{2}$ microcolumns. The highest number of the identified phosphopeptides was achieved when we packed small pipet tips with $3 \mathrm{~mm}$ of the $\mathrm{TiO}_{2}$ resins (Figure 3-1 B).

\subsubsection{Impact of fractionation on the number of identified phosphopeptides}

In order to decrease the complexity of the peptide mixture and increase the yield of phosphopeptides identification, a fractionation step is commonly used upstream of LC-MS analysis. Strong cation exchange (SCX) has been used before as a complementary step for the phosphopeptides enrichment (Villen and Gygi, 2008). In this method, the negatively charged phosphopeptides in the acidic condition have a lower affinity for the negatively charged resin of the column than the corresponding non-phosphorylated peptides. Therefore, phosphopeptides are enriched in the earlier fractions (Grimsrud et al., 2010). Here, I have observed that using strong cation exchange following by $\mathrm{TiO}_{2}$ significantly increased the number of the identified phosphosites. Note that a substantial amount of unique phosphosites was identified up to the fifth fraction (Figure 3-2 A). 
A

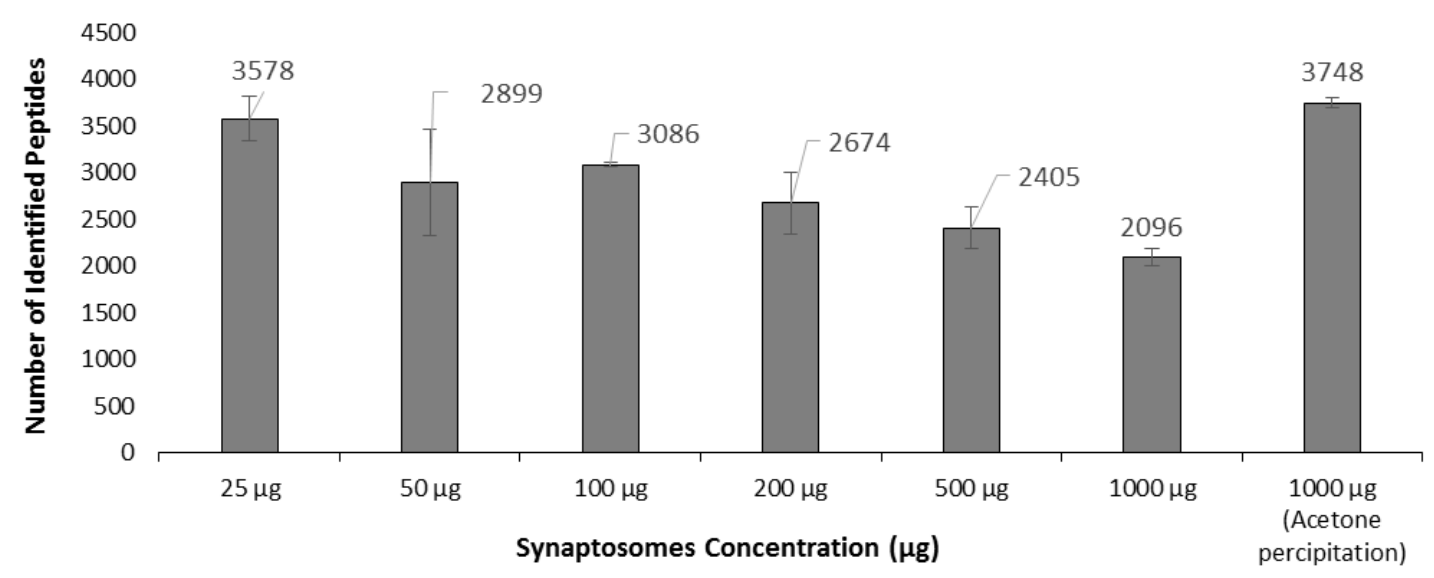

B
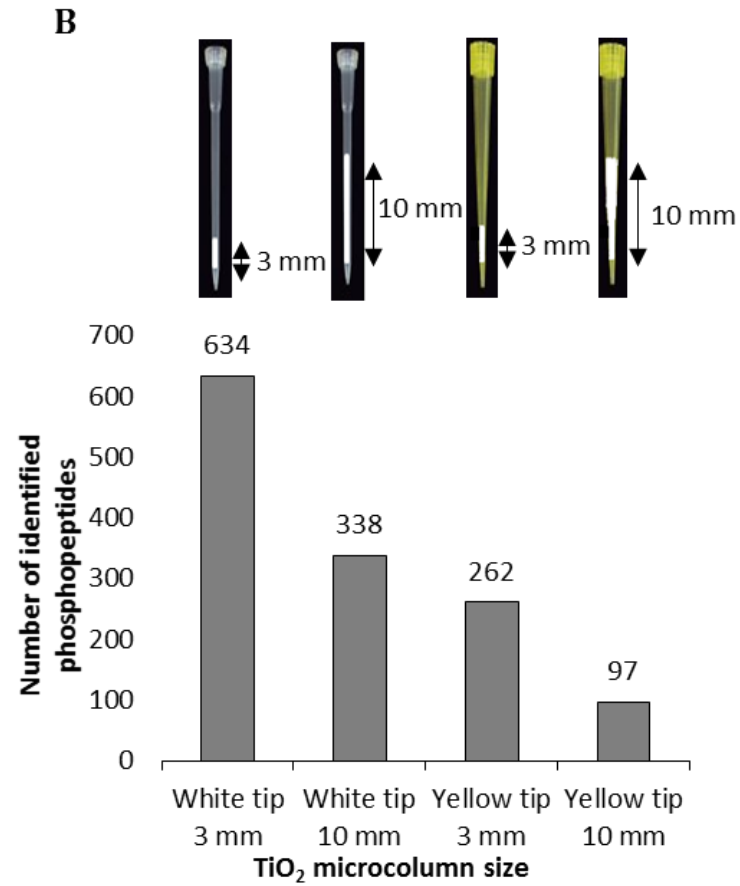

Figure 3-1: Optimization of protein digestion and $\mathrm{TiO}_{2}$ phosphopeptides enrichment.

(A) Different amount of purified synaptosomes were used for the tryptic digestion reaction (trypsin: protein of 1:50). Protein were solubilized in $10 \mu \mathrm{l}$ of $1 \%$ RapiGest. The more starting material resulted in the lower number of identified peptides. A large improvement of peptide identification was observed upon protein precipitation by $100 \%$ acetone overnight. Data are represented as mean \pm SEM, $n=3$. (B) The different sizes of the $\mathrm{TiO}_{2}$ microcolumns were used to enrich phosphopeptides. The highest number of phosphopeptides was identified by using the smallest microcolumn. 
A

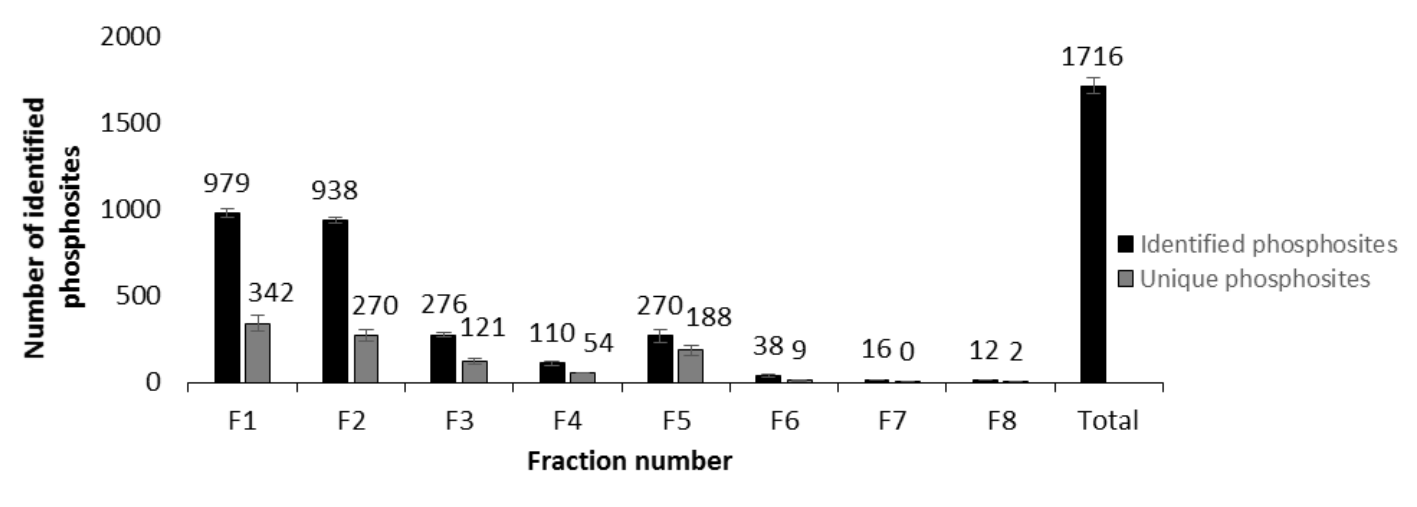

B

Distribution of multiple phosphorylated peptides

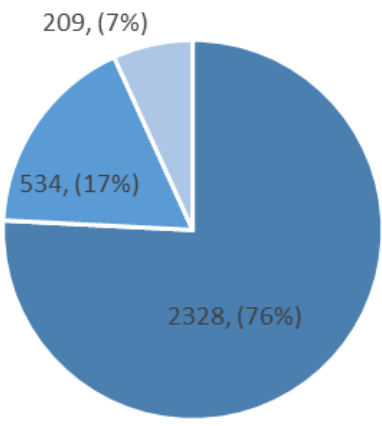

- Monophosphorylated

- Diphosphorylated

- Triphosphorylated
C Distribution of phosphorylated amino acids

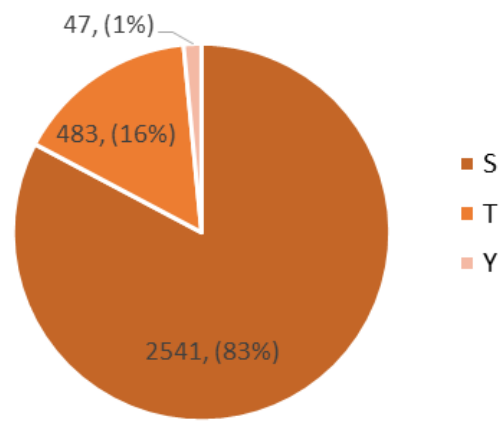

Figure 3-2: Effect of strong cation exchange chromatography on the identification of phosphosites.

(A) Peptides resulted from digestion of one mg of synaptosome were subjected to the strong cation exchange chromatography. The first 8 fractions were collected. Phosphopeptides in each fraction were enriched by $\mathrm{TiO}_{2}$ method and analyzed by mass spectrometer separately. Fractionation by SCX resulted in significantly higher identification of the phosphopeptides. Data are represented as mean \pm SEM, $n=3$. (B) Distribution of multiple phosphorylated peptides. More than $75 \%$ of the peptides contain 1 phosphosite. (C) Distribution of phosphorylated amino acids. Serine (S) phosphorylation dominated the identified phosphosites followed by threonine (T). Only $1 \%$ of the identified phosphosites were phospho-tyrosines (Y).

In the next step, I have checked the distribution of the phosphopeptides based on the multiple phosphorylated sites and amino acids. It was revealed that most of the peptides are monophosphorylated and only $17 \%$ and $7 \%$ of them were di and triply phosphorylated, respectively. Regarding amino acids, serine phosphorylation was dominant over threonine and tyrosine phosphorylation. Indeed, only $1 \%$ of the identified phosphosites were phosphorylated at tyrosine (Figure 3-2 B and C). 
In similar phosphoproteomics studies of the brain, higher amount of the starting material was used in order to identify more phosphosites (Villen and Gygi, 2008, Trinidad et al., 2012). Here, I have decided to test whether increasing the amount of starting material will result in an overall higher number of phosphosite identification. Therefore, I used $10 \mathrm{mg}$ of isolated synaptosomes and solubilized them in $8 \mathrm{M}$ urea instead of RapiGest due to its high cost. The solubilized synaptosomal proteins were digested by trypsin. Urea was removed by C18 desalting column and digested peptides were subjected to SCX fractionation and $\mathrm{TiO}_{2}$ enrichment. It was observed that increasing the starting material 10 fold resulted in almost 2 fold increase in phosphosite identification (Figure 3-3). Taken together the marginal improvement and low practicality of 10-fold increase in the starting material, digestion of 1 mg of synaptosomes in RapiGest was used as a standard protocol for all experiments further in this thesis.

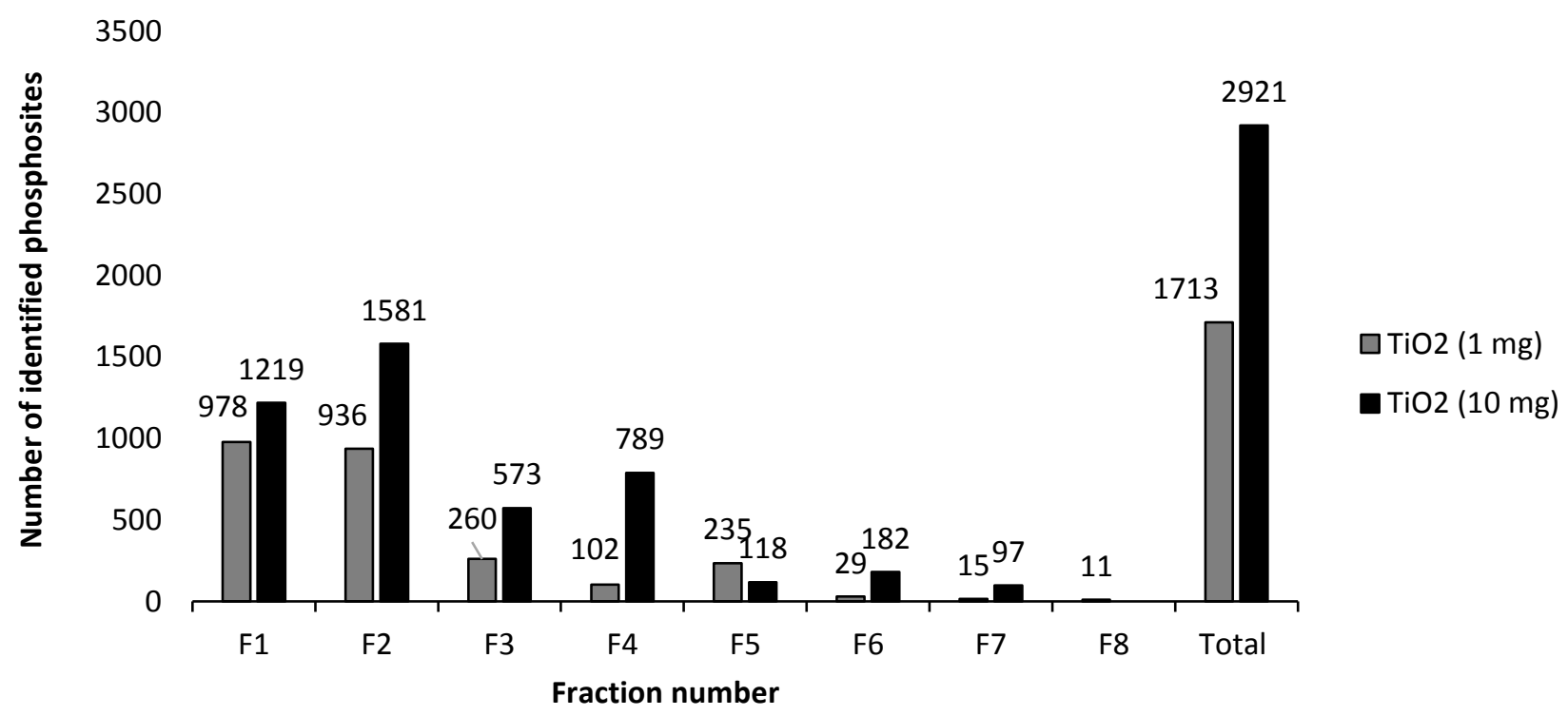

Figure 3-3: Effect of starting material on the identification of phosphopeptides.

1 and $10 \mathrm{mg}$ of synaptosomes were digested and subjected to $\mathrm{SCX}+\mathrm{TiO}_{2}$ enrichment. Increasing the starting material 10 fold increased the number of identified phosphosites less than 2 fold. 
In the next step, I have tested Immobilized Metal Affinity Chromatography (IMAC) as an alternative phosphopeptides enrichment method to the $\mathrm{TiO}_{2}$ method. In this method, the positively charged metal ions (in this case Fe(III)) were chelated on the solid phase iminodiacetic acid resin and interacted with the negatively charged phosphoryl group of the phosphosites. Similar to the $\mathrm{TiO}_{2}$ method, the binding and washing buffer had low $\mathrm{pH}$ to reduce binding of the carboxylic acid group of non-phosphorylated peptides to the resin. The elution of the phosphopeptides was performed by high pH buffer. Here, I have used $20 \mathrm{mg}$ of synaptosomes, solubilized in $8 \mathrm{M}$ urea and digested with trypsin. The digested peptides were fractionated by SCX and divided into two fractions. The first fraction was enriched by $\mathrm{TiO}_{2}$ and the second fraction by IMAC. This procedure was carried out in 3 biological replicates. Here, I have observed that on average the number of the identified phosphosites by IMAC was higher than $\mathrm{TiO}_{2}$. However, the number of the identified phosphosites by $\mathrm{TiO}_{2}$ enrichment method was much more robust than IMAC (see the error bars of Figure 3-4). Therefore, I have decided to continue the enrichment by $\mathrm{TiO}_{2}$ rather than IMAC.

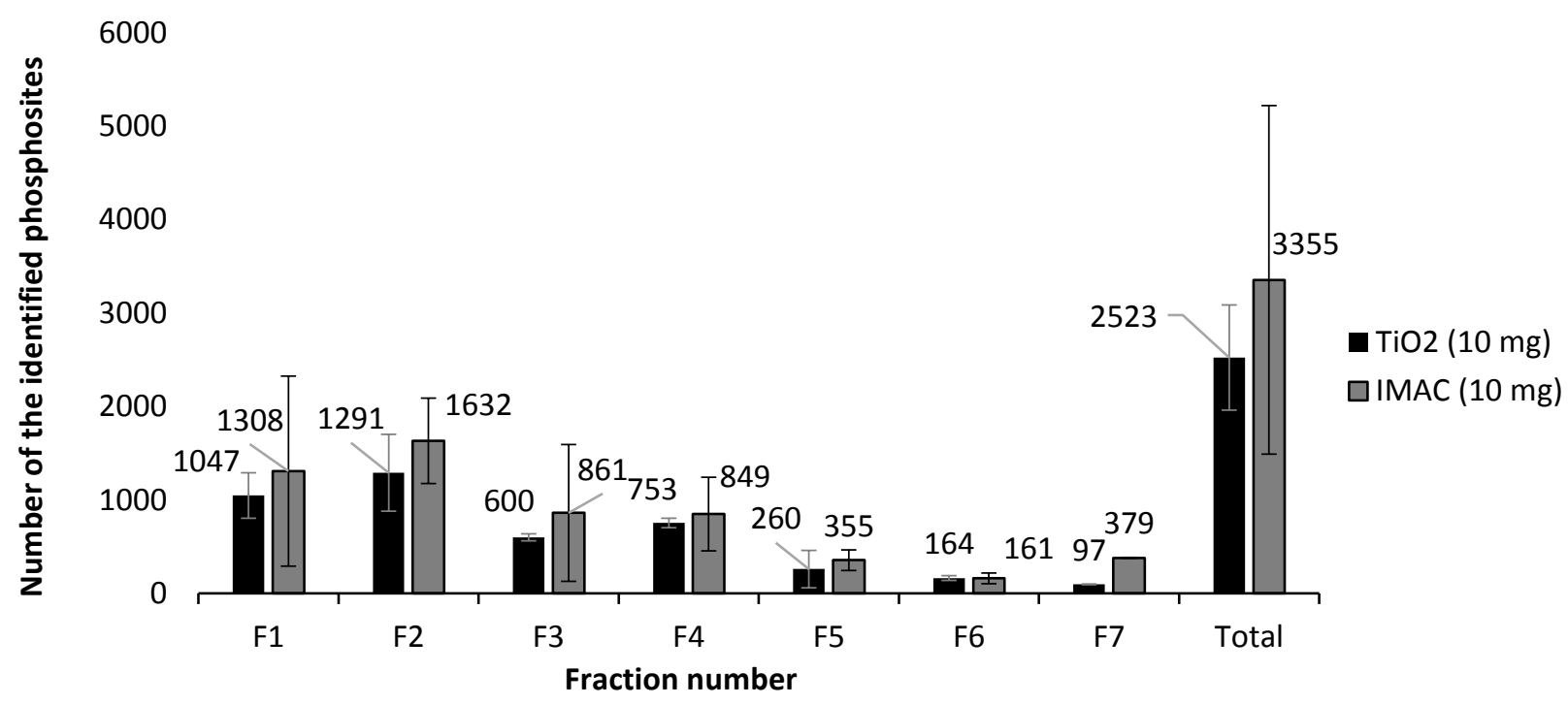

Figure 3-4: Comparison between $\mathrm{TiO}_{2}$ and IMAC phosphopeptides enrichment methods.

$10 \mathrm{mg}$ of synaptosomes were digested and subjected to $\mathrm{SCX}+\mathrm{TiO}_{2}$ and $\mathrm{SCX}+\mathrm{IMAC}$ enrichment methods. On average higher number of phosphosites were identified by SCX+IMAC method. However, $\mathrm{SCX}+\mathrm{TiO}_{2}$ method deliver more robust and reproducible number of identified phosphosites. Data are represented as mean \pm SEM, $\mathrm{n}=3$. 


\subsubsection{Stable isotopic labeling}

In recent years, most of the proteomics experiments are focused on the differential quantification of the protein/peptide abundance under different treatment conditions. Similarly, in this study, we aimed to relatively quantify the phosphopeptides of differentially treated synaptosomes, using the dimethyl labeling as the labeling method. In this method, deuterium-formaldehyde and sodium cyanoborohydride label the $\mathrm{N}$-terminus and the $\varepsilon$ amino group of the Lys amino acid through the reductive amination (Hsu et al., 2003), resulting in the mass shift of 4 Da between light and medium and 8 Da between light and heavy labeled peptides. Here we tested the efficiency of the dimethyl labeling by dividing an identical digested sample to two fractions and labeled them differently by heavy and light dimethyl labeling. Then the two fractions were mixed together and were analyzed by MS. The results revealed that (Figure 3-5) more than 94\% of the peptides and $93 \%$ of the phosphopeptides were labeled correctly and accurately $(-0.5<\log 2$ ratio $\mathrm{H} / \mathrm{L}<0.5)$. Therefore, I used the dimethyl labeling as the method of choice for the relative peptides quantification.

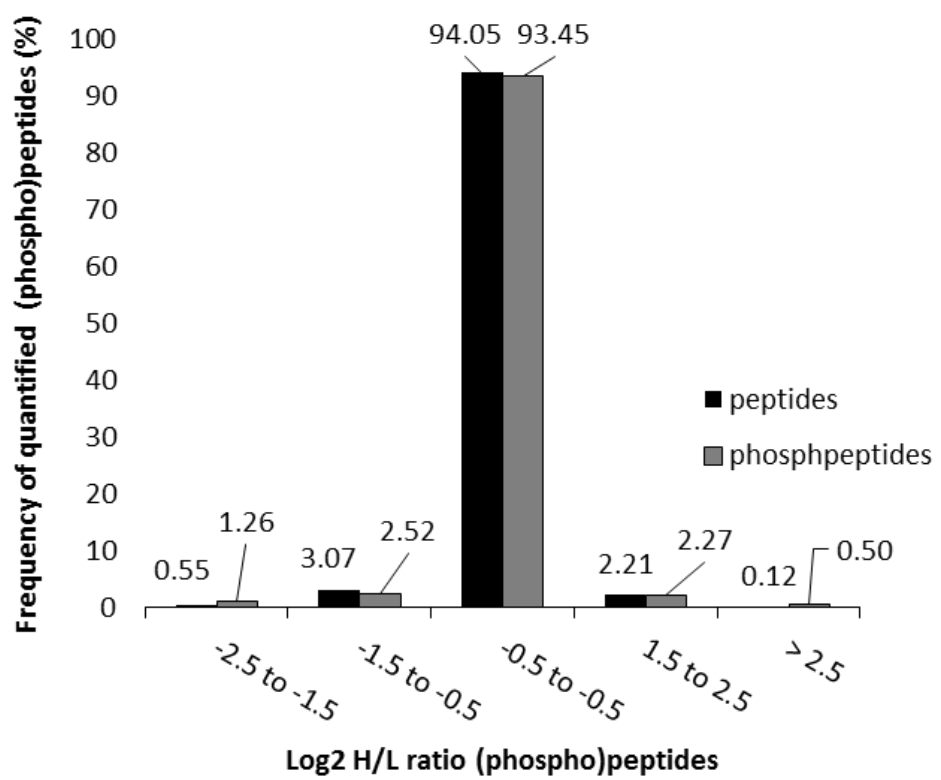

Figure 3-5: Dimethyl labeling.

Dimethyl labeling robustly labeled more than $93 \%$ of the (phospho) peptides (log2 heavy/light ratio between 0.5 and -0.5 ). 


\subsubsection{Impact of dimethyl labeling on the number of quantified phosphosites}

In the next step, I have investigated how the dimethyl labeling affects the number of the quantified phosphopeptides. For this purpose, I have digested $2 \times 1 \mathrm{mg}$ of synaptosomes and performed dimethyl labeling on one of the samples, whereas the second sample remained unlabeled. Next, I have subjected both the dimethyl labeled sample and the non-labeled sample to the $\mathrm{SCX}$ and $\mathrm{TiO}_{2}$ enrichment and analyzed them by mass spectrometer (Figure 36 A). As it is shown in the Figure 3-6 B, dimethyl labeling cuts down the number of quantified phosphosites by half in comparison to the identified phosphosites in the label-free approach. This is due to the fact that the complexity of the sample mixture at the MS1 level will be increased by the labeling. Also, for the quantification by dimethyl labeling both heavy and light versions of phosphopeptides have to be identified while in the label-free method identification of the single unlabeled phosphopeptide is required. Although dimethyl labeling decreases the number of the quantified phosphopeptides, I have decided to use it as the quantification method due to its more accurate phosphopeptide quantification when compared to the label-free approach. 
A

\section{Dimethyl labeling}

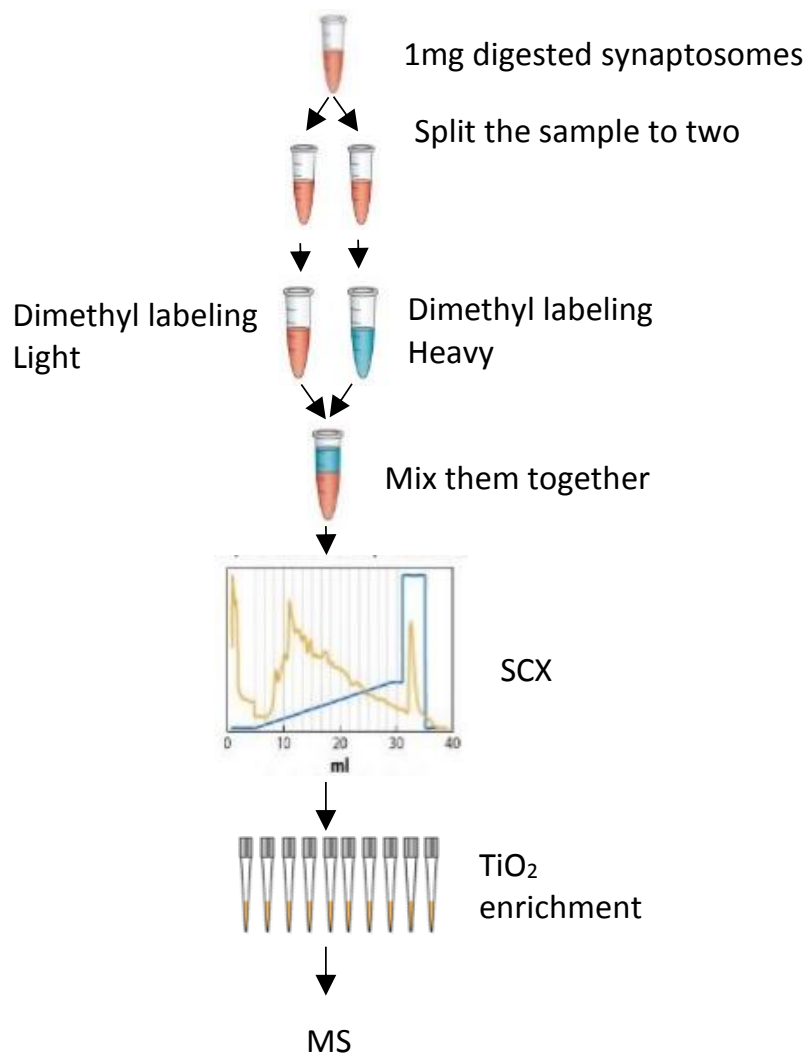

Label free
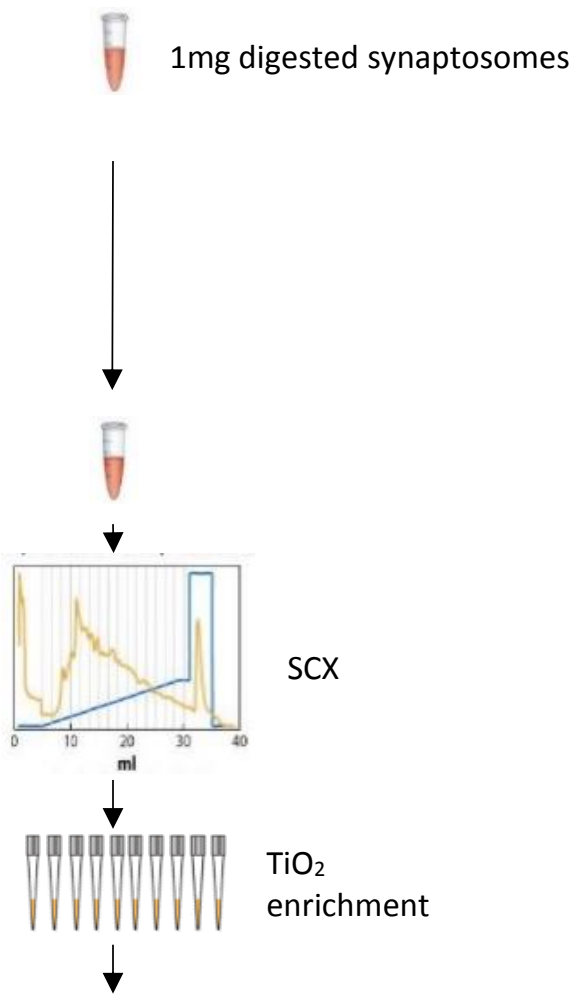

MS

B

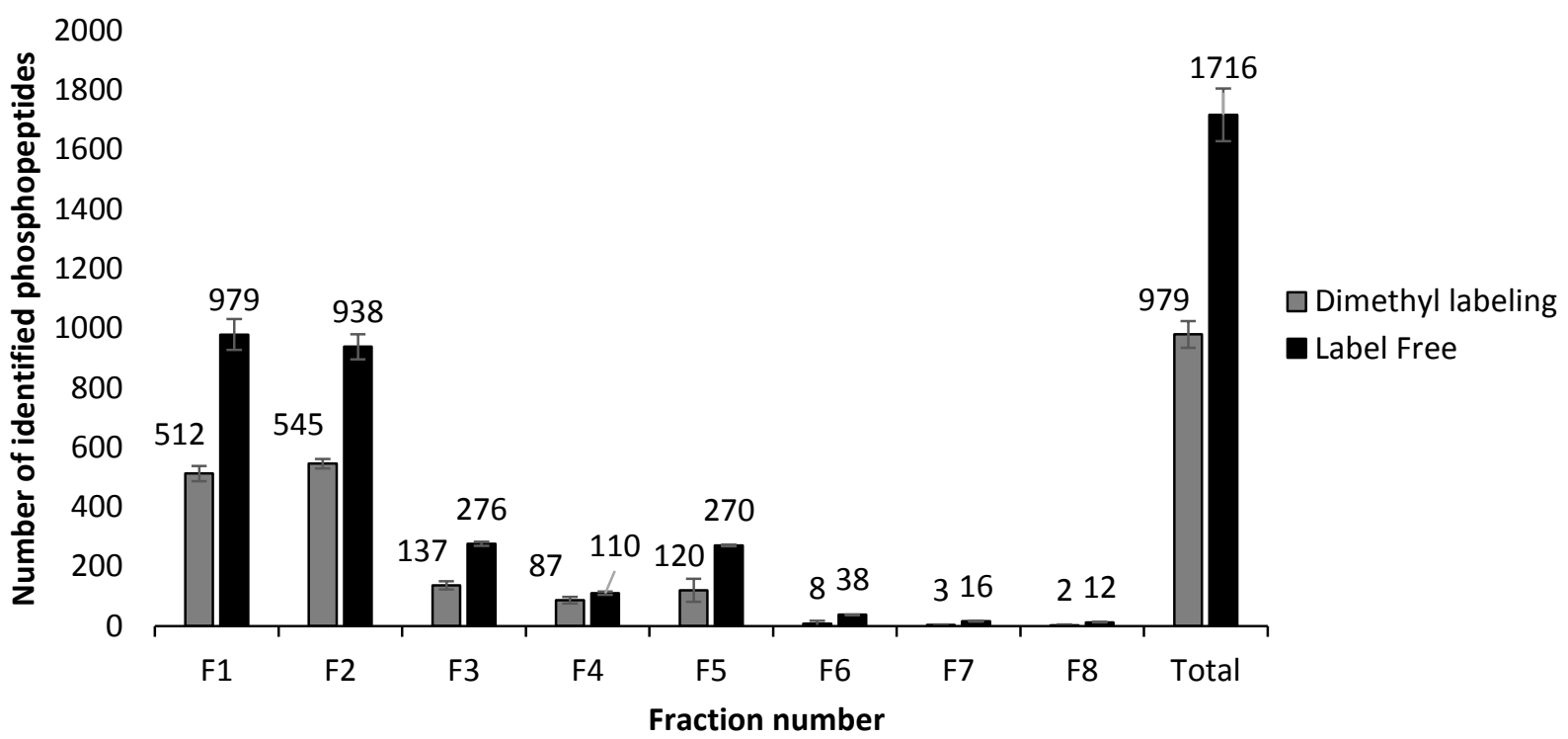

Figure 3-6: Effect of dimethyl labeling on the number of quantified phosphosites.

(A) $2 \times 1 \mathrm{mg}$ digested synaptosomes were subjected to the label free and dimethyl labeling workflow as depicted in figure. (B) The number of identified phosphosites in label free approach was two times higher than quantified phosphosites by dimethyl labeling. Data are represented as mean \pm SEM, $n=3$. 


\subsection{Phosphoproteomics of isolated nerve terminals under different stimulation conditions}

As it was mentioned in section 1.5 , the main aim of this project was to investigate the changes of the phosphorylation status of presynaptic proteins upon stimulation. For this analysis, isolated nerve terminals, (synaptosomes) were used. Synaptosomes were prepared from 56 weeks old rats using a standard subcellular fractionation protocol (see Material and Method). To stimulate synaptosomes, high concentration of $\mathrm{KCl}(50 \mathrm{mM})$ was used. $\mathrm{KCl}$ stimulation results in the clamped depolarization of the plasma membrane and the release of the glutamate. Glutamate release is fast in the first seconds and reaches a plateau almost 2 min after stimulation (Figure 3-7). In this study the samples were collected for the further analysis by MS 2 min after stimulation to capture the activated steady-state status of the exoand endocytotic machinery.

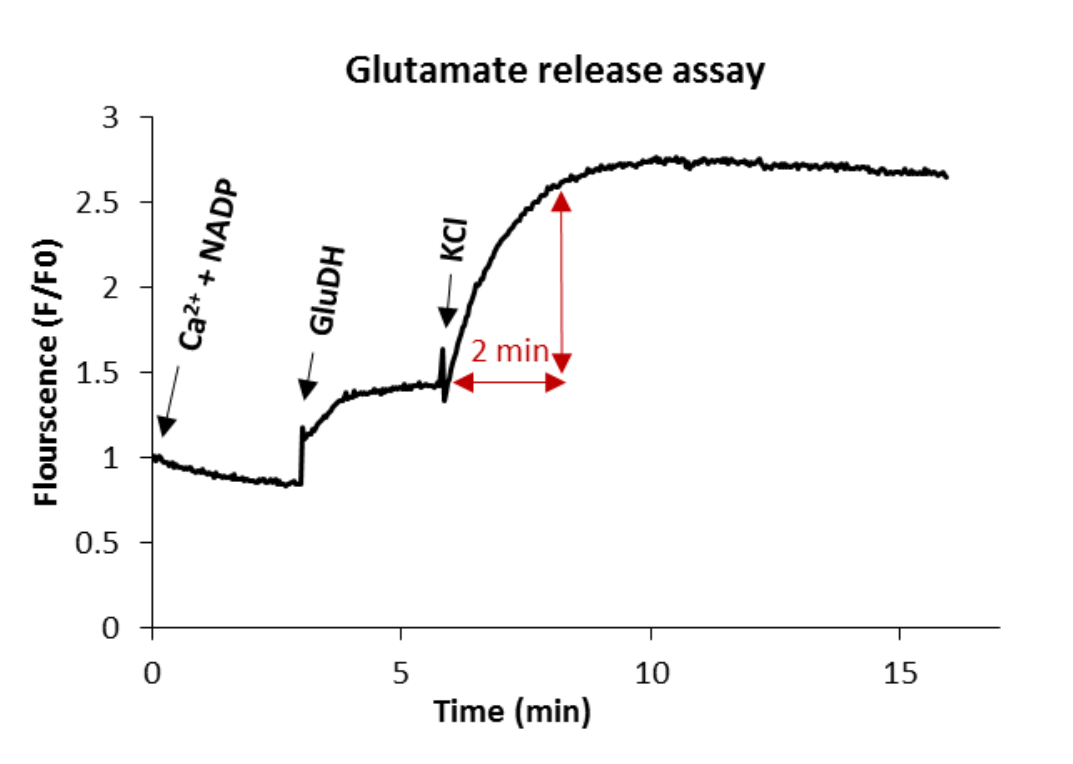

Figure 3-7: Glutamate release assay.

Representative trace of NADPH-fluorescence in suspended synaptosomes during subsequent addition of glutamate dehydrogenase (GluDH) to convert glutamate and NADP to a-ketoglutarate and NADPH. KCl was added to stimulate exocytotic release of glutamate. Synaptosomes were pre-incubated in sodium buffer for 5 $\min$ at $37^{\circ} \mathrm{C}$. Then, they were transferred to a cuvette, followed by the addition of $\mathrm{CaCl}_{2}$ (final concentration 1.3 $\mathrm{mM}$ ) and NADP (final concentration $1.3 \mathrm{mM}$ ) to the reaction. The baseline was measured for $3 \mathrm{~min}$, then GluDH (200 U) was added. The measurement was carried on for another $3 \mathrm{~min}$. Synaptosomes were stimulated by addition of $\mathrm{KCl}(50 \mathrm{mM})$. The functional and responsive synaptosomes released the glutamate at least 2.5 folds higher than the baseline. Samples for the MS analysis were collected 2 min after $\mathrm{KCl}$ stimulation. 


\subsubsection{Functional analysis of synaptosomes}

To ensure that each preparation of isolated synaptosomes used for phosphoproteome analysis is responsive to stimulation and their phosphorylation machineries (kinases and phosphatases) are functional, synaptosomes were examined by two quality checks prior to sample collection for the MS analysis. First, we measured glutamate release by $\mathrm{KCl}$ stimulation (Figure 3-8 A). As expected, after addition of $\mathrm{KCl}\left(\mathrm{K}^{+}\right)$, synaptosomes that were pre-incubated in the presence of $\mathrm{Ca}^{2+}\left(\mathrm{K}^{+}, \mathrm{Ca}^{2+}\right)$ released glutamate more and faster than in the absence of $\mathrm{Ca}^{2+}\left(\mathrm{K}^{+}, \mathrm{EGTA}\right)$. The glutamate release in the presence of calcium is a result of exocytosis whereas the release in the absence of $\mathrm{Ca}^{2+}$ is due to non-exocytotic release resulting from the reversal of glutamate transporters in the plasma membrane that depend on the membrane potential (Nicholls and Sihra, 1986). Also, we observed that release of glutamate remained at the baseline without $\mathrm{KCl}$ stimulation (EGTA).

Second, the phosphorylation status of two well-characterized presynaptic phosphosites (synapsin1 S603, and dynamin1 S774) was monitored upon stimulation using phosphospecific antibodies. Synapsin1 S603 and dynamin1 S774 are known to be phosphorylated and dephosphorylated upon stimulation, respectively (Jovanovic et al., 2001, Graham et al., 2007). As shown in the Figure 3-8 B, phosphorylation of both sites changed as expected in all replicates, confirming that synaptosomes are functional and the workflow preserves the phosphorylation status of the proteins.

\subsubsection{Activity-dependent phosphorylation changes of presynaptic proteins}

It is known that arrival of the action potential to the nerve terminals opens the voltage-gated calcium channels via depolarization of the membrane and results in the influx of $\mathrm{Ca}^{2+}$ ions to the nerve terminal. This triggers synaptic vesicles to fuse with the membrane and release the neurotransmitter (Sudhof, 2013). To distinguish between the $\mathrm{Ca}^{2+}$-dependent and depolarization-dependent phosphorylation changes of presynaptic proteins, three stimulation conditions were designed as follows:

(i) Depolarization in the presence of calcium ions $\left(\mathrm{K}^{+}, \mathrm{Ca}^{2+}\right)$

(ii) Depolarization in the absence of calcium ions ( $\left.\mathrm{K}^{+}, \mathrm{EGTA}\right)$

(iii) No depolarization (EGTA) 

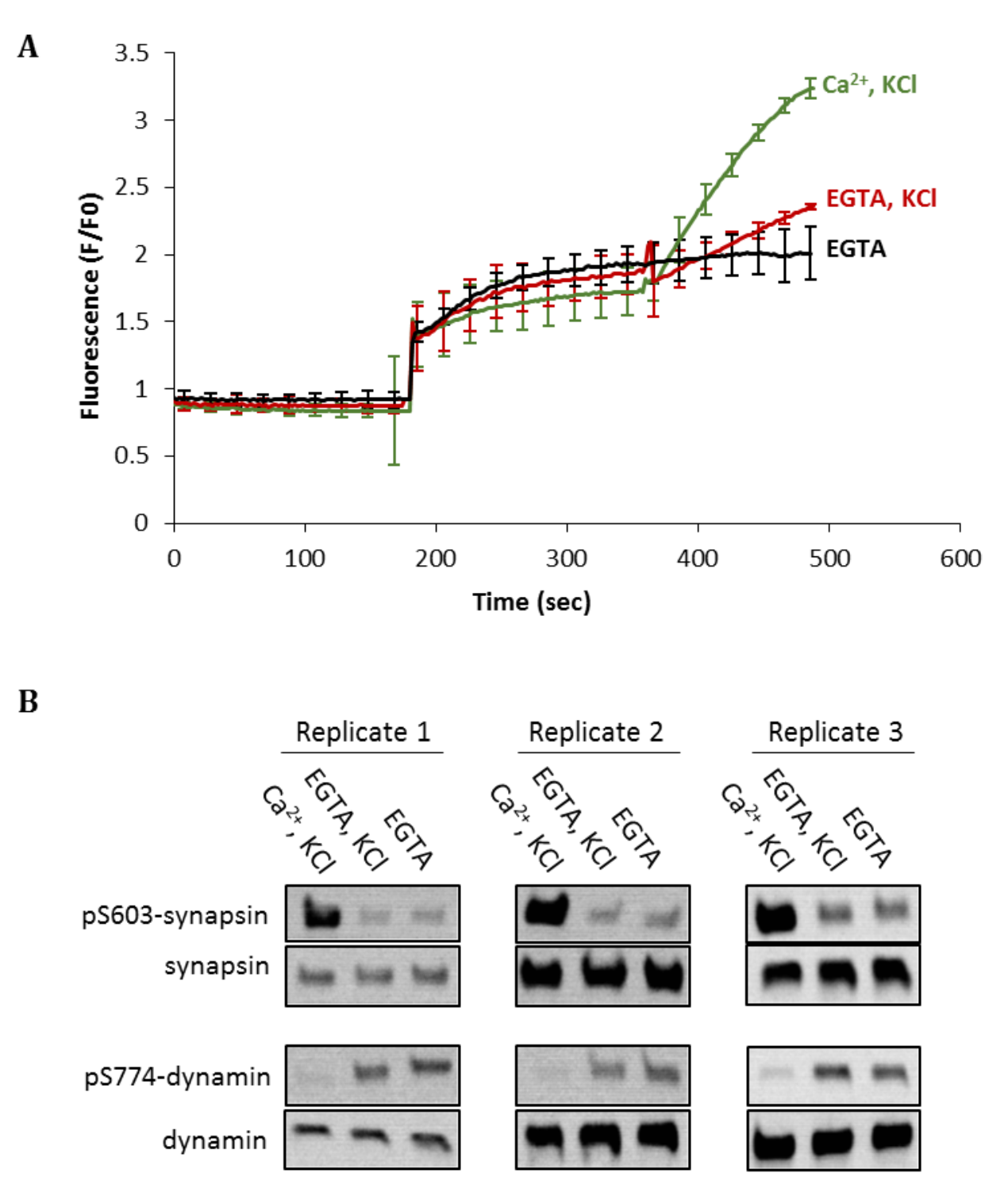

Figure 3-8: Functional assessment of synaptosomes

(A) Glutamate release was measured when synaptosomes were stimulated by $\mathrm{KCl}$ in the presence of calcium $\left(\mathrm{Ca}^{2+}, \mathrm{KCl}\right)$, the absence of calcium (EGTA, KCl) and when synaptosomes were not stimulated (EGTA). The release of glutamate was at the highest level when synaptosomes were stimulated in the presence of calcium. It was decreased in the absence of calcium and was at the lowest level when synaptosomes were not stimulated. Data represented as mean of three replicates, with the bars indicating the range of values.

(B) Immunoblot analysis of two representative presynaptic proteins. Synaptosomes were collected 2 min after stimulation in the 3 above-mentioned conditions for immunoblot analysis with phospho-specific antibodies against synapsin Ser603 (pS603) and dynamin Ser774 (pS774). Upon stimulation in the presence of calcium, synapsin Ser603 and dynamin Ser774 phosphorylation were up and downregulated, respectively. For the loading control antibodies against synapsin and dynamin that were not sensitive to the phosphorylation sites were used, adapted from (Kohansal-Nodehi et al., 2016). 
Treated synaptosomes in the three mentioned stimulation conditions were collected 2 min after stimulation and lysed by lysis buffer to stop the reaction. The same amount of synaptosomes for each condition was acetone precipitated and digested by trypsin. Tryptic peptides were then labeled differentially (Heavy and Light) by stable isotopes using dimethyl labeling. To elucidate the phosphorylation changes of the proteins in each stimulation condition compared to the other two conditions, the differentially labeled peptides were mixed in 1:1 ratio as it is shown in Figure 3-9. Then the mixed peptides were subjected to SCX. The first twelve fractions were collected, and the phosphopeptides in each fraction were enriched by $\mathrm{TiO}_{2}$ approach and analyzed by mass spectrometer separately. The mentioned comparisons between conditions are listed below:

\begin{tabular}{|l|l|l|}
\hline \# of comparison & Abbreviation & To investigate... \\
\hline Comparison 1 & $\mathrm{~K}^{+}, \mathrm{Ca}^{2+}$ vs K+, EGTA & $\mathrm{Ca}^{2+}$-dependent phosphorylation changes \\
\hline Comparison 2 & $\mathrm{~K}^{+}, \mathrm{Ca}^{2+}$ vs EGTA & $\mathrm{Ca}^{2+}$ and depolarization-dependent phosphorylation changes \\
\hline Comparison 3 & $\mathrm{~K}^{+}$, EGTA vs EGTA & Depolarisation-dependent phosphorylation changes \\
\hline
\end{tabular}

All comparisons were performed in three biological replicates. All the phosphosites with the following criteria were filtered out for the further data analysis (See the Material and Method section 2.2.2.7).

- Phosphosites that are quantified in only in one replicate

- Phosphosites with localization probability less than 0.75

- Phosphosites that are identified in the decoy database search (reverse phosphosites).

Due to the rigorous selection criteria, the phosphosites that were reliably quantified in the comparison 1, 2 and 3 were 67.9\%, 62.5\%, and 58.85\%, respectively, of the total phosphosites that were initially quantified. The regulated phosphosites in comparison 1, 2, 3 are listed in the Appendices section Tables 6-1, 6-2 and 6-3, respectively. For the list all quantified phosphosites in comparison 1, 2, 3 see the supplementary Tables S1, S2, and S3. 


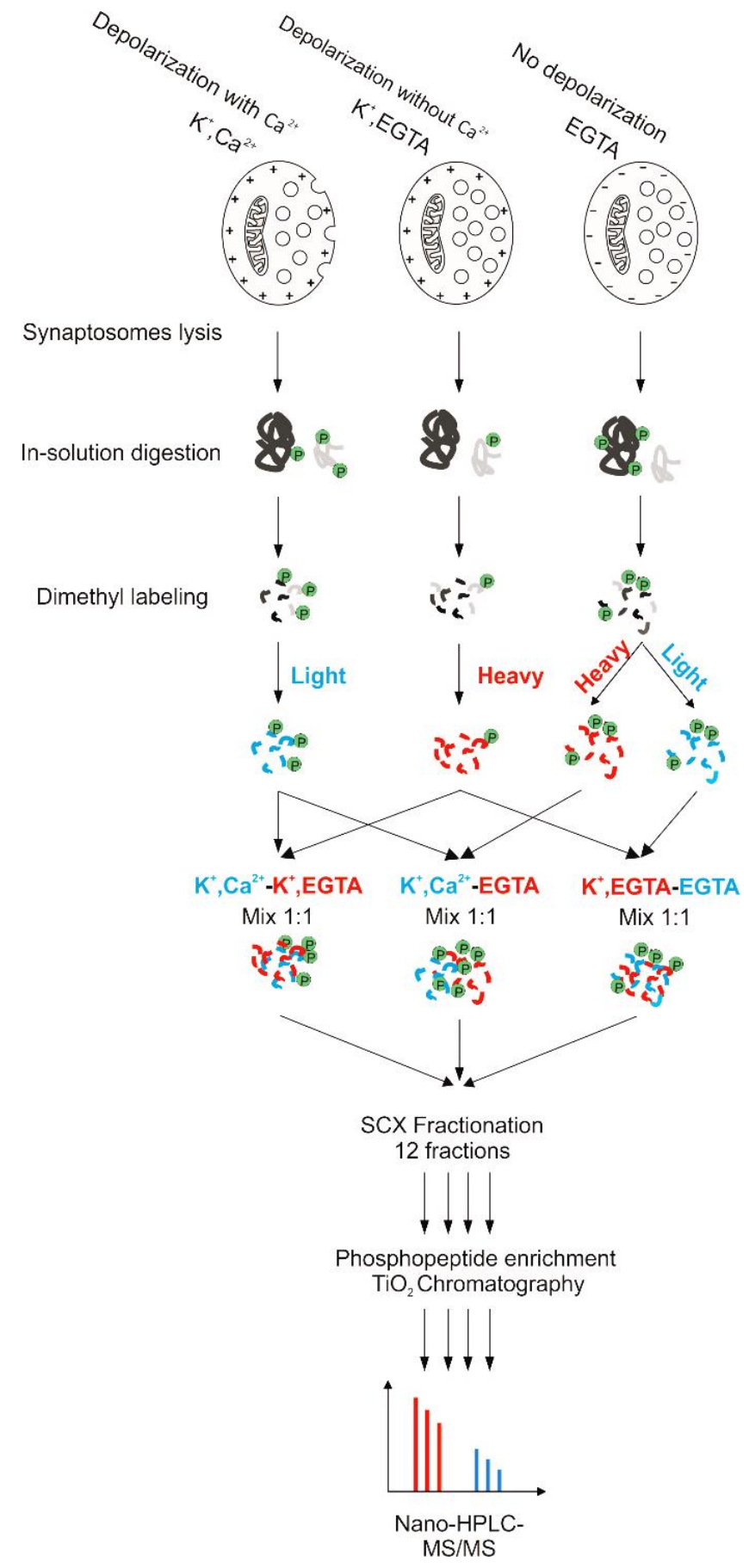

Figure 3-9: Experimental workflow for quantitative phosphoproteomics of synaptosomes in different stimulation conditions.

Synaptosomal proteins treated in the mentioned conditions above $\left(\mathrm{K}^{+}, \mathrm{Ca}^{2+}\right),\left(\mathrm{K}^{+}, \mathrm{EGTA}\right)$ and (EGTA) were extracted by lysis buffer followed by acetone precipitation. The protein pellets were dissolved in $1 \%$ RapiGest and digested by trypsin. Next, digested peptides were labeled by stable isotope dimethyl labeling. To obtain the pair-wise comparison between the conditions, differentially labeled peptides were mixed at 1:1 ratio and subjected to strong cation exchange chromatography. The first 12 fractions were collected and each fraction was separately enriched for phosphopeptides by $\mathrm{TiO}_{2}$ and analyzed by LC-MS/MS, adapted from (KohansalNodehi et al., 2016). 
Distribution of the phosphosites in each comparison is shown in Figure 3-10 A, B, C. To determine the significantly up or downregulated phosphosites, we have used 'significant A' test, embedded in 'Perseus', the software that was designed to analyze the proteomics data. This test determines the phosphosites with significant $\log 2$ ratio $\mathrm{H} / \mathrm{L}$ outliers relative to a total population of the phosphosites (p-value $\leq 0.05$ ). Remarkably, in the first two comparisons ( $\mathrm{Ca}^{2+}$ ions are involved in one of the conditions, Figure 3-10 A and B) dynamics of the phosphorylation changes is higher than in the third comparison (no $\mathrm{Ca}^{2+}$ ions, Figure 3-10 C). In fact, in the third comparison even the phosphosites that are determined to be significant outliers, are closely clustered around 1:1 ratio (or $\log 2=0$ ). Note that synapsin 1 S603 and dynamin1 S774 were quantified as up and downregulated phosphosites in the first two comparisons (Figure 3-10 A, B) which agree with the western blot analysis by phosphospecific antibodies (Figure 3-8 B), confirming the high quality of the dataset.

Next, the overlap of the phosphosites in the three comparisons was investigated. A very high overlap of the total quantified phosphosites was observed between the three replicates (Figure 3-11 A). Also, a substantial overlap of regulated phosphosites between depolarization with $\mathrm{Ca}^{2+}$ versus depolarization without $\mathrm{Ca}^{2+}$ and depolarization with $\mathrm{Ca}^{2+}$ versus no depolarization was observed (Figure 3-11 B, C and D). Intriguingly, phosphosites that are common in the first two comparisons (these phosphosites are shown in the Figure 3-11 B with the black oval) showed an identical tendency in regulation and similar fold changes (Figure 3-11 E). In addition, the phosphosites regulated in the third comparison showed almost no overlap with the regulated phosphosites in the other two conditions. These results suggest that different sets of phosphosites are affected by $\mathrm{Ca}^{2+}$ and membrane depolarization, respectively. 

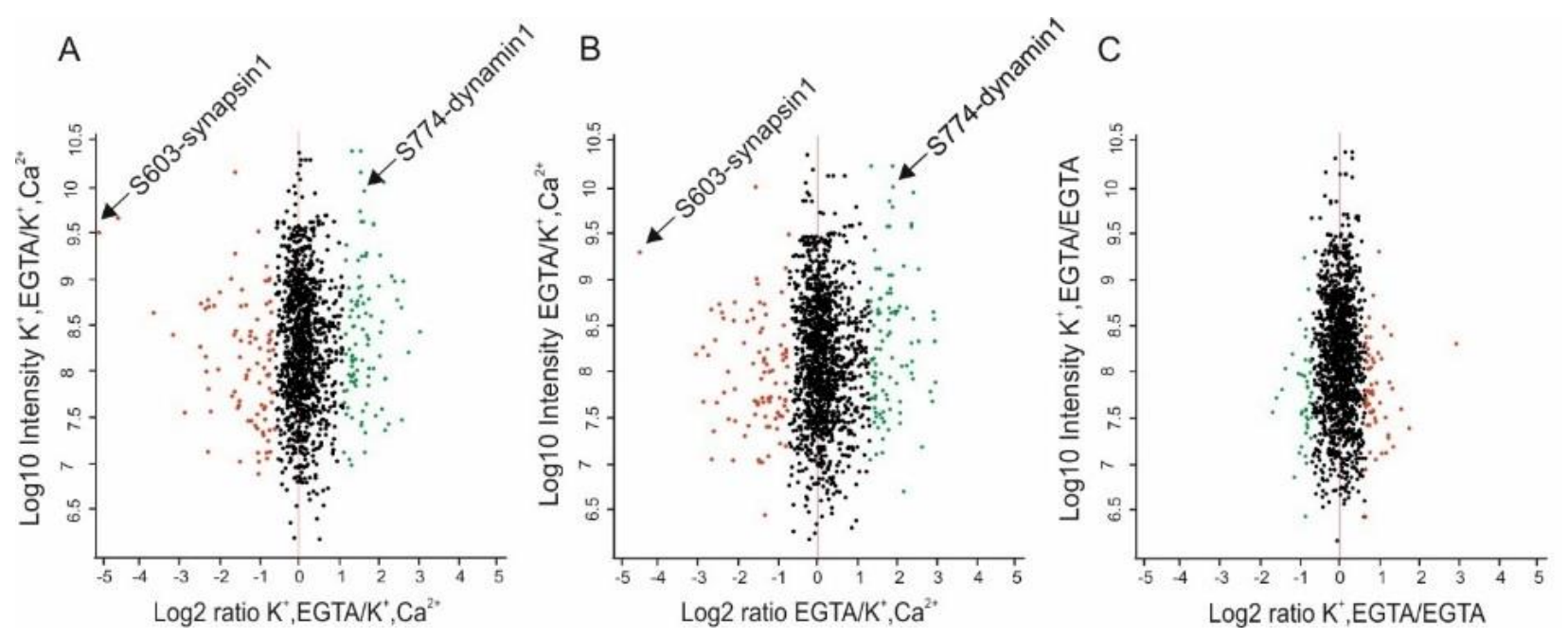

\begin{tabular}{|c|c|c|c|}
\hline Number of & $\mathrm{K}^{+}, \mathrm{Ca}^{2+} \mathrm{vs} \mathrm{K}^{+}$, EGTA & $\mathrm{K}^{+}, \mathrm{Ca}^{2+}$ vs EGTA & $\mathrm{K}^{+}$, EGTA vS EGTA \\
\hline Quantified phosphosites & 1373 & 1243 & 1371 \\
\hline Upregulated phosphosites & 93 & 95 & 44 \\
\hline Downregulated phosphosites & 75 & 81 & 52 \\
\hline Quantified phosphoproteins & 524 & 502 & 525 \\
\hline Upregulated phosphoproteins & 54 & 59 & 37 \\
\hline Downregulated phosphoproteins & 28 & 49 & 38 \\
\hline
\end{tabular}

Figure 3-10: Overview of the quantified phosphosites in the three comparisons.

Distribution of quantified phosphosites from pair-wise comparisons of $(A)$ depolarization with $\mathrm{Ca}^{2+}$ versus depolarization without $\mathrm{Ca}^{2+}\left(\mathrm{K}^{+}, \mathrm{Ca}^{2+}\right.$ versus $\left.\mathrm{K}^{+}, \mathrm{EGTA}\right)(\mathrm{B})$ depolarization with $\mathrm{Ca}^{2+}$ versus no depolarization $\left(\mathrm{K}^{+}, \mathrm{Ca}^{2+}\right.$ versus EGTA) and (C) depolarization without $\mathrm{Ca}^{2+}$ versus no depolarization ( $\mathrm{K}^{+}$, EGTA versus EGTA). Stable isotope dimethyl labeling was used for the quantification. To determine significantly regulated phosphosites in each comparison the two-tailed 'Significant A' test ( $p$-value $\leq 0.05$ ) of the Perseus module was used (See Material and Method). (D) The number of quantified up- or down-regulated phosphosites and phosphoproteins in the three comparisons. In the first two comparisons (A and B) red/green and black dots represent the upregulated/downregulated and the not regulated phosphosites, respectively, in the $\mathrm{K}^{+}, \mathrm{Ca}^{2+}$ conditions compared to $\mathrm{K}^{+}$, EGTA or EGTA conditions. In the third comparison (C) the red/green and the black dots represent the upregulated/downregulated and the not regulated phosphosites, respectively, in the $\mathrm{K}^{+}$, EGTA conditions compared to EGTA condition. Note that in the comparisons involving the calcium ions (A and B) the regulation of the phosphosites upon stimulation is more pronounced (more regulated phosphosites, with the $\log 2$ ratio distanced further from 0). The position of the synapsin S603 and dynamin S774 are shown as the up and downregulated phosphosites, respectively. In the comparisons were calcium ions were excluded (C), the number of the regulated phosphosites was low compared to two other comparisons (A and B) and the log2 of the ratio was clustered around 0, adapted from (Kohansal-Nodehi et al., 2016). 

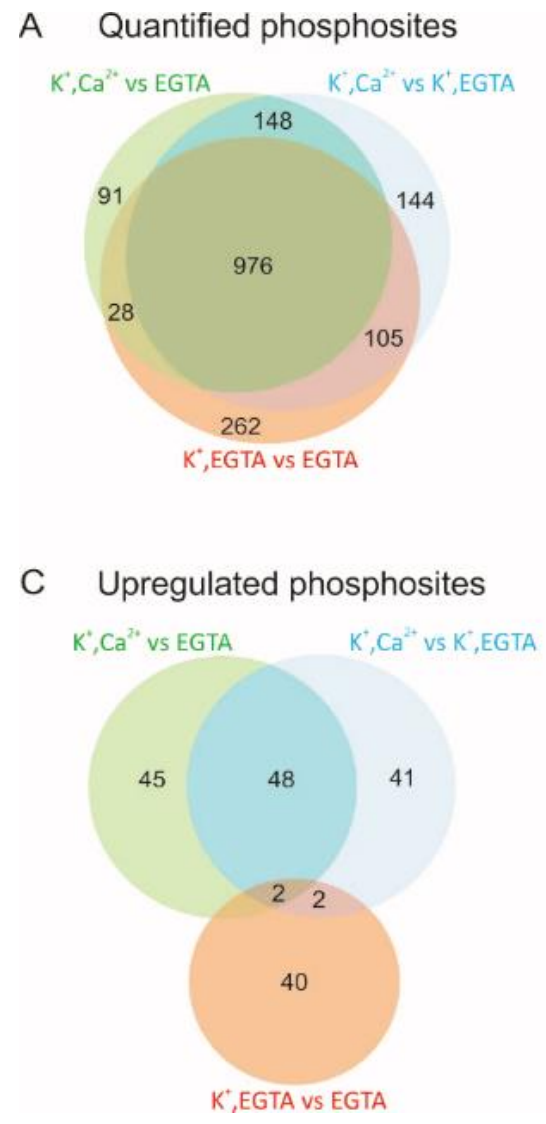

B Regulated phosphosites

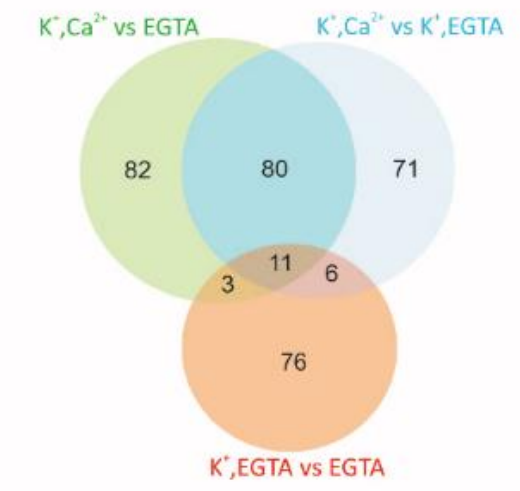

D Downregulated phosphosites

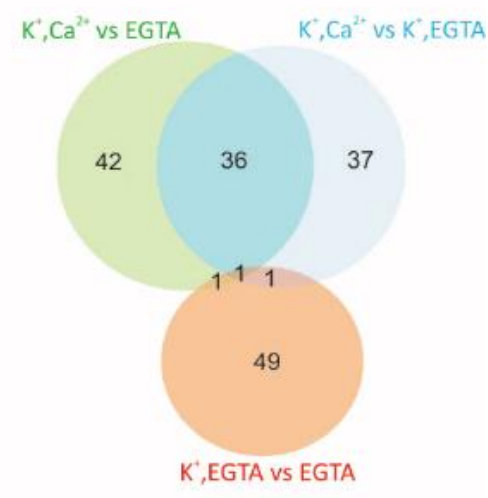

$\mathrm{E}$

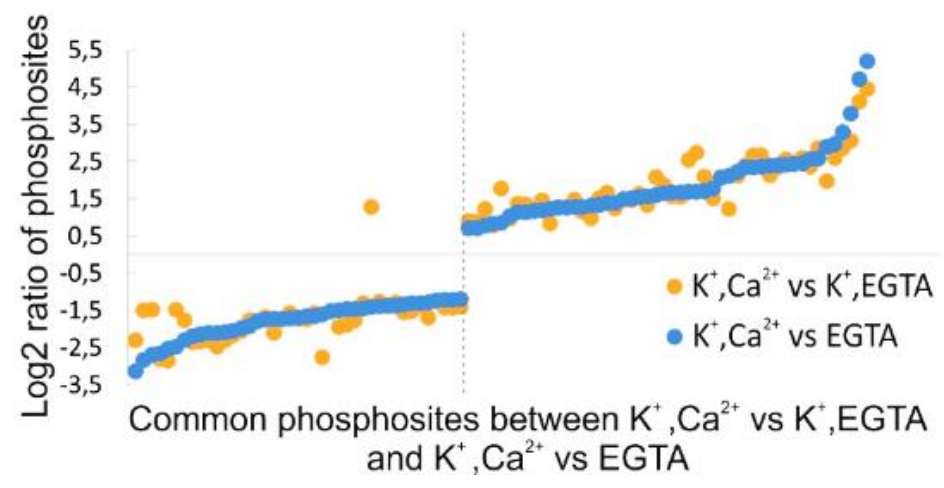

Figure 3-11: Overlap of quantified phosphosites in different comparisons.

The proportional Venn diagram between (A) all quantified phosphosites, (B) regulated phosphosites, (C) upregulated phosphosites and (D) downregulated phosphosites in all three comparisons. Although the overlap between all the quantified phosphosites in all comparisons was substantial (A), the overlap of regulated phosphosites (up or down) was close to $50 \%$ between $\mathrm{K}^{+}$, $\mathrm{Ca}^{2+}$ versus $\mathrm{K}^{+}$, EGTA and $\mathrm{K}^{+}, \mathrm{Ca}^{2+}$ versus EGTA comparisons and almost no overlap between $\mathrm{K}^{+}$, EGTA versus EGTA and the other two comparisons was observed (C and D).

(E) The plot depicting the extent of regulation of phosphosites that were identified both in $\mathrm{K}^{+}, \mathrm{Ca}^{2+}$ versus $\mathrm{K}^{+}$, EGTA and $\mathrm{K}^{+}, \mathrm{Ca}^{2+}$ versus EGTA comparisons (phosphosites are shown with the black oval in B). An almost identical direction of phosphosites regulation (up or downregulation) was observed. Also, the log2 ratios of dimethyl labeling between the phosphosites were very similar between the two comparisons, adapted from (Kohansal-Nodehi et al., 2016). 


\subsubsection{Functional/localization grouping of regulated phosphosites}

Our analysis revealed that a large fraction of the quantified phosphosites was not changed upon stimulation. This attracts a special interest to the fraction of regulated phosphosites and their involvement in the regulation of synaptic transmission. In order to gain a better overview of the functional properties of the regulated phosphosites in the three comparisons, we have annotated proteins manually and categorized them based on their function and localization into 15 groups (Figure 3-12) according to a previous study (Boyken et al., 2013). With few exceptions (e.g translation/transcription or metabolism of neurotransmitters), the majority of proteins containing the regulated phosphosites are known to have a role in synaptic vesicle trafficking or in neurotransmitter signaling. In the same line, groups such as mitochondrial proteins that are known to constitute a large fraction of presynaptic proteins (Boyken et al., 2013) have very low number of regulated phosphosites. This proves that our experimental approach is specifically targeting the synaptic transmission related proteins and phosphorylation of other proteins present in synaptosomes (such as metabolism related proteins) remains unregulated.

In summary, identification of the regulated phosphosites upon stimulation presented in this study provides novel insights into the molecular regulation of neurotransmitter release. In the following sections, the groups of proteins with the highest number of regulated

phosphosites in the $\mathrm{K}^{+}, \mathrm{Ca}^{2+}$ versus $\mathrm{K}^{+}$, EGTA comparison and the proteins that are known to be involved in synaptic vesicle recycling will be discussed in more detail.

\subsubsection{Active zone proteins}

In our study, component of active zone protein group showed extensive changes in their phosphorylation status upon stimulation. In Figure 3-13 amino acid positions of the quantified phosphosites of active zone proteins are shown. Opposite changes (up and downregulation) were detected among phosphosites of this group upon stimulation. Between the 7 proteins of this group, bassoon and piccolo are massively phosphorylated with 48 and 31 quantified phosphosites, respectively. 


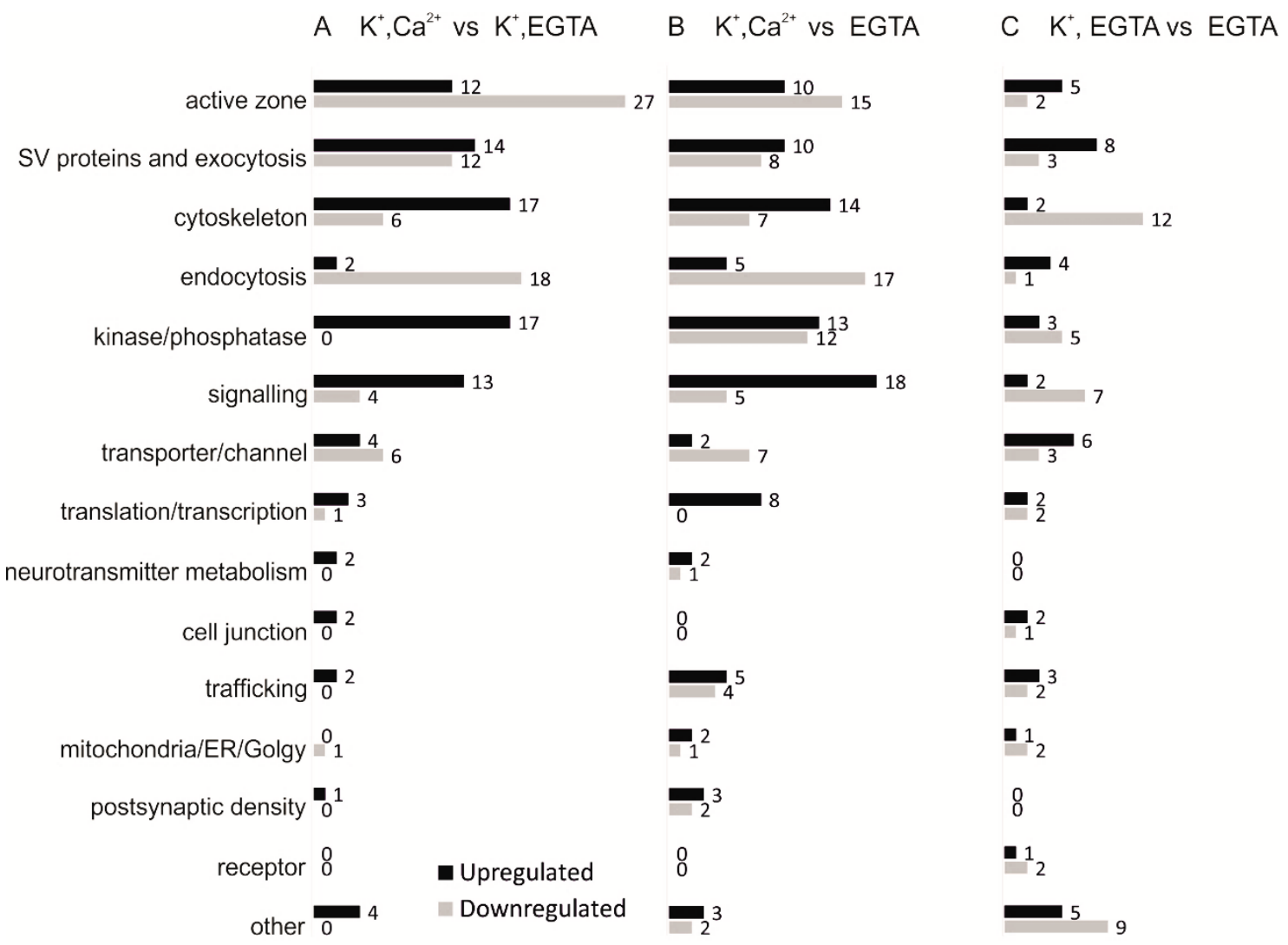

Figure 3-12: Functional/localization categorization of the regulated phosphosites in the three comparisons.

Regulated phosphosites were manually categorized based on their function and localization into 15 groups according to the previous publication (Boyken et al., 2013). Proteins with other functions/localizations than listed or uncharacterized proteins were grouped as 'other' category, adapted from (Kohansal-Nodehi et al., 2016).

Although bassoon and piccolo are homologous proteins, some differences were observed between their phosphorylation patterns. For example, phosphosites of bassoon were dramatically downregulated upon stimulation (16 phosphosites), whereas only 4 phosphosites of piccolo were downregulated and many of them remained not regulated. Also, 5 phosphosites of bassoon and 2 of piccolo were upregulated. While downregulation of majority of the phosphosites can serve as a regulator of the global properties of the protein such as surface charge, the upregulated phosphosites might have particular physiological significance. For example, it is known that interaction of bassoon with the adaptor protein 14-3-3 is dependent on the phosphorylation of bassoon of S2844 (Schroder et al., 2013). 
RIM is known as the central organizer of the active zone. It binds to Munc13 via its N-terminal zinc finger (Deng et al., 2011) and with the helices surrounding the zinc finger to Rab3a and Rab27 (Wang et al., 2000, Fukuda, 2003). These interactions at the N-terminal of RIM links synaptic vesicle to the active zone and to the priming factor Munc13. RIM also binds to Nand P/Q-type $\mathrm{Ca}^{2+}$ channels (Kaeser et al., 2011a) and ELKS proteins (Ohtsuka et al., 2002) via its PDZ domain and the proline-rich domain located between $\mathrm{C} 2 \mathrm{~A}$ and $\mathrm{C} 2 \mathrm{~B}$. Via C2B domain, RIM binds to the liprin-a3 and synaptotagmin 1 (Schoch et al., 2002) and via C2A domain to SNARE proteins (Coppola et al., 2001). In our study, 16 phosphosites of RIM were quantified (4 upregulated and 3 downregulated, Figure 3-13). One of the upregulated phosphosites (S413) was reported to be the substrate of PKA and its role in the long-term plasticity has been investigated extensively (Lonart et al., 2003, Kaeser et al., 2008). The other upregulated phosphosite S346 is in the proximity of zinc finger (FYVE2) domain which suggests its possible role in the binding of RIM to Munc13 or Rab3a. S742 and S745 located between the PDZ domain and the C2A are downregulated upon stimulation. Dephosphorylation of these sites might have a role in the interaction of RIM with $\mathrm{Ca}^{2+}$ channels or SNARE proteins.

Munc13 is considered as the priming factor of the active zone and is essential for synaptic transmission (Chen et al., 2013). In our dataset, we quantified 2 phosphosites at the Nterminus of the Munc13a (S255 and S259) that were not regulated upon stimulation. The Nterminus of the Munc13 is known to be important for its interaction with RIM (Betz et al., 2001).

The function of liprin- $a 3$ in the active zone is not completely understood but there is some evidence that it is needed for the formation of active zones and the recruitment of synaptic vesicles and LAR-type receptor phosphotyrosine phosphatases (Dai et al., 2006, Stryker and Johnson, 2007). In this study, 4 phosphosites of liprin-a3 were quantified (1 up, 1 down and 2 not regulated). 


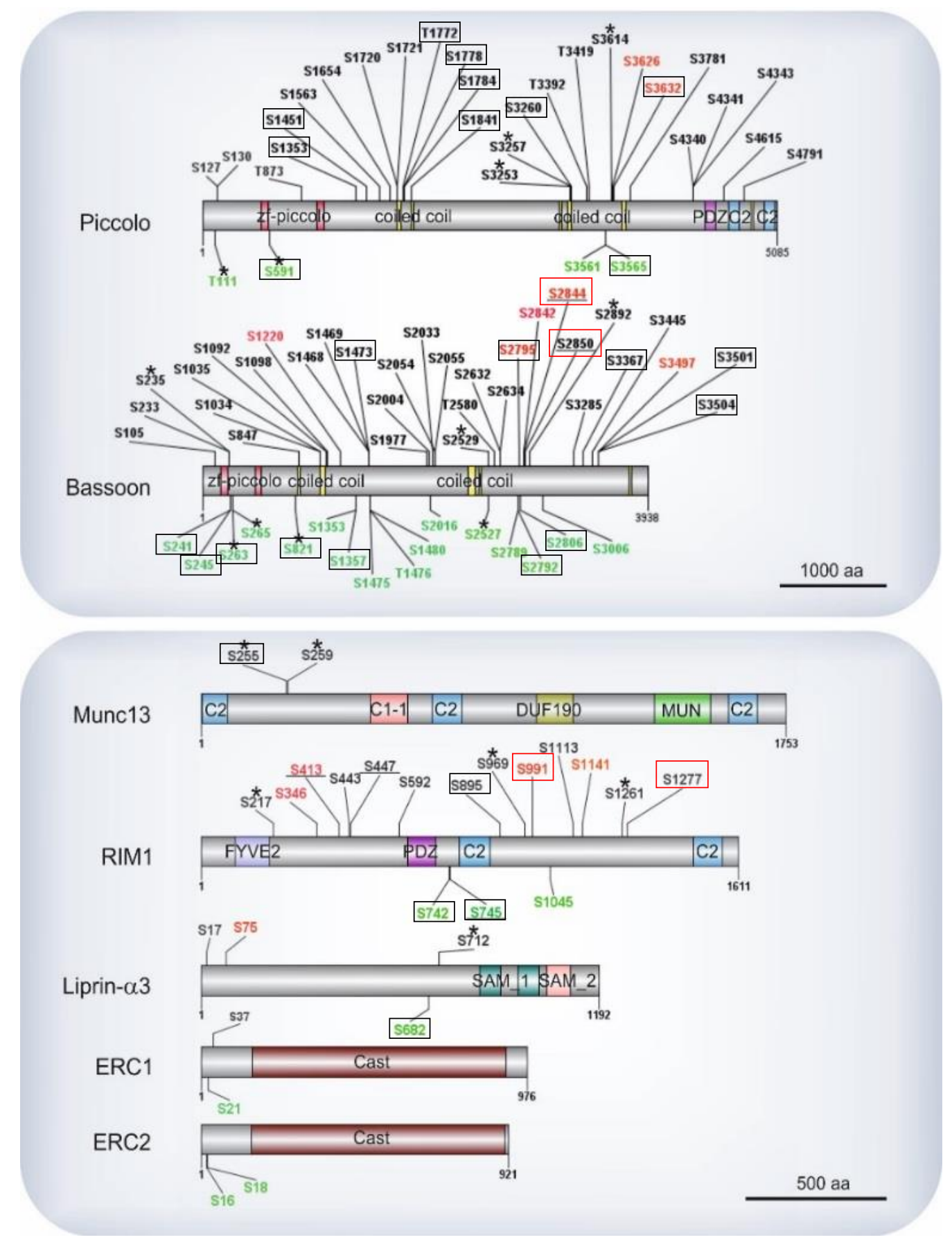

Figure 3-13: Quantified phosphosites of active zone in the $\mathrm{K}^{+}$, $\mathrm{Ca}^{2+}$ versus $\mathrm{K}^{+}$, EGTA comparison.

Main protein components of the active zone with the phosphosites quantified in this study are depicted. Red/green and black color represent respectively the up/down and not regulated phosphosites in $\mathrm{K}^{+}, \mathrm{Ca}^{2+}$ versus $\mathrm{K}^{+}$, EGTA comparison. Phosphosites that are reported to be physiologically relevant are underlined. Unmarked phosphosites are identified in the previous proteomic discovery-mode mass spectrometry analysis (source: publicly available PhosphoSitePlus database). Phosphosites with asterisk represent phosphosites reported in this study for the first time. Phosphosites that are surrounded with the black/red box are the putative substrates of Calcineurin/CaMKII (see result chapter, part 3-3), adapted from (Kohansal-Nodehi et al., 2016) 
ERC proteins, also known as CAST or ELKS, are important for the regulation of the size of the ready releasable pool of synaptic vesicles (Kaeser et al., 2009). We quantified two phosphosites in N-terminus of ERC2 (S16 and S18) that were 6 and 7 fold downregulated, respectively. Interestingly, a recent publication (Held et al., 2016) showed that the decrease of the readily releasable pool via ERC proteins is due to its $\mathrm{N}$-terminal interaction with liprina3 and bassoon.

\subsubsection{Endocytosis proteins}

After exocytosis, synaptic vesicles are retrieved from the membrane mainly by the clathrinmediated endocytosis pathway. Different studies have shown that proteins involved in this process undergo rapid and coordinated dephosphorylation upon $\mathrm{Ca}^{2+}$ influx into the nerve terminal through voltage-gated calcium channels. These proteins are collectively called 'dephosphins' and consist of dynamin1, amphiphysins1 and 2, epsin, eps15 and AP180. Dephosphins are not structurally related and function in different stages of synaptic vesicle endocytosis. In our dataset, we have quantified 64 phosphosites belonging to endocytosisrelated proteins, and 20 of them were changed. Consistent with the previous report, the majority of the significantly altered phosphosites (18 phosphosites) were downregulated (Figure3-14 A).

Dynamin1 is a GTPase that binds to the neck of budded vesicles and mediates the fission of synaptic vesicles from the membrane. It is known that dynamin binds to SH3 domains of proteins such as intersectin, amphiphysin and endophilin through its proline-rich domain (PRD) at the C-terminus (Sundborger and Hinshaw, 2014). In our dataset, we have quantified 9 phosphosites of dynamin1, all close to the PRD domain, and 6 of which were dephosphorylated upon stimulation (Figure3-14 B). It is known that phosphorylation of dynamin 1 by PKC and CdK5 modulates its interaction with other endocytotic proteins (Tomizawa et al., 2003b) as well as membrane lipids (Liu et al., 1994).

Amphiphysins are scaffold proteins that link the early stage endocytotic proteins such as AP2 and clathrin to the late stage proteins like dynamin and synaptojanin (Cousin and Robinson, 2001). We have quantified 9 phosphosites of amphiphysin1, none of them are significantly regulated. However, a slight downregulation of some phosphosites (S245, S249, S239) around proline-rich domain (PRD) was detected. It is known that amphiphysin 1 binds to 
dynamin and synaptojanin through PRD domain. Therefore it can be suggested that these sites may have some implications in amphiphysin protein-protein interactions (Craft et al., 2008). We have also quantified 2 phosphosites of amphiphysin2 (Bin1) that are significantly downregulated.

A

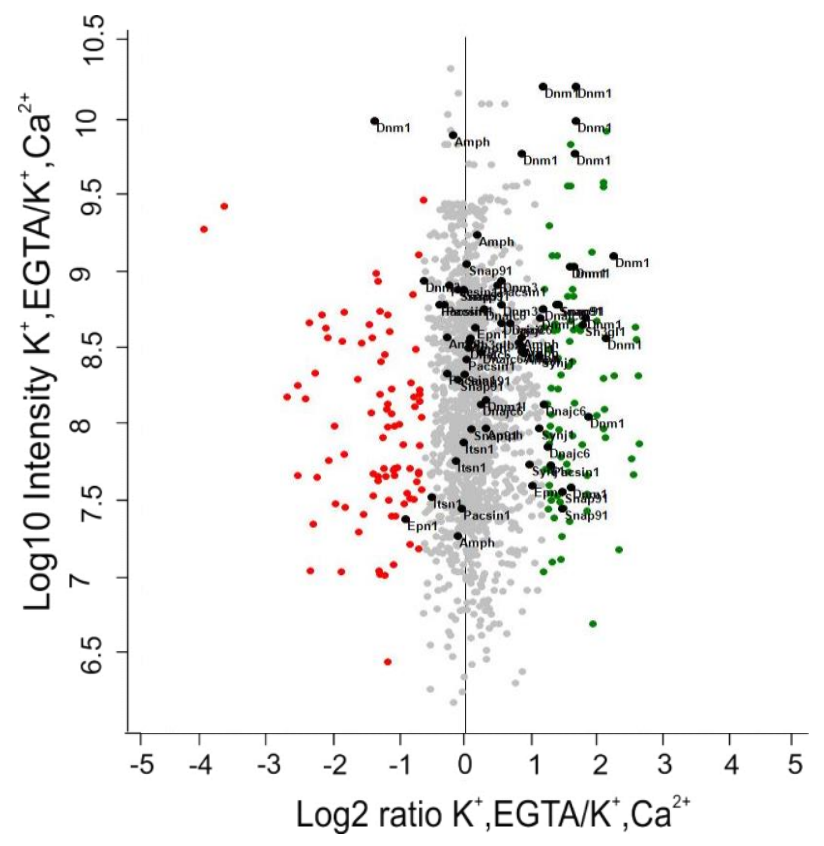

B

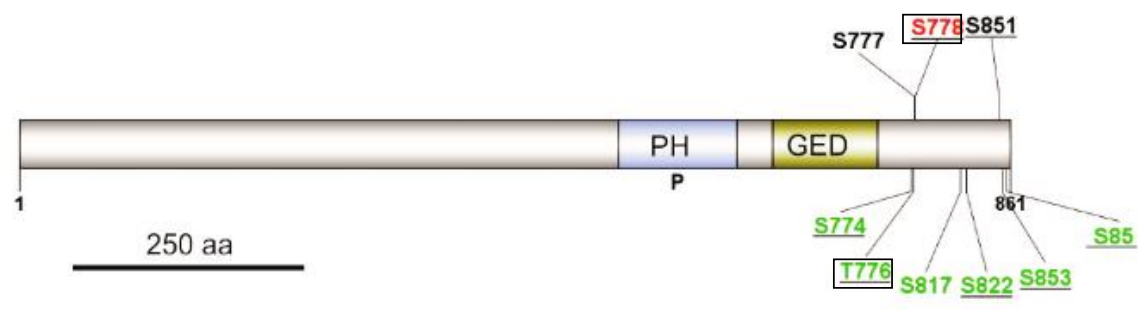

Figure 3-14: Phosphosites related to clathrin-mediated endocytosis.

(A) The graph is depicting the distribution of the phosphosites of proteins involved in clathrin-mediated endocytosis quantified in $\mathrm{K}^{+}, \mathrm{Ca}^{2+}$ versus $\mathrm{K}^{+}$, EGTA comparison. Except for two phosphosites all the quantified phosphosites are either not regulated or down regulated upon stimulation in the presence of calcium.

(B) Quantified phosphosites of dynamin1 as a representative of the endocytotic proteins. Dominant downregulation of the phosphosites upon stimulation in the C-terminus of the protein was observed. Phosphosites that are reported to be physiologically relevant are underlined. Phosphosites that are surrounded with the black box are the putative substrates of Calcineurin.

Epsin1 is another member of dephosphins that is reported to be dephosphorylated upon stimulation. In our study, we have quantified 3 phosphosites of epsin-1. One of them, in contrast to previous reports (Chen et al., 1999), was upregulated upon stimulation. It is known that phosphorylation of epsin decreases its interaction with AP2 (Chen et al., 1999). 
AP180 is located under the outer clathrin shell of coated vesicles and links the clathrin shell to the vesicle membrane (Hao et al., 1999). In our dataset, 9 phosphosites of AP180 were quantified and 3 of them were downregulated (S600, S621, and S627). It is known that AP180 binds to another clathrin coat assembly protein, AP2, via the patch of amino acids between positions 623 and 680 (Hao et al., 1999). Interestingly, all the three downregulated phosphosites in our study are close to this region of the protein. This suggests that dephosphorylation of AP180 may potentially regulate its interaction with AP2.

Synaptojanin is a phosphatidylinositol polyphosphate (PtdInsP) phosphatase that mediates the uncoating of synaptic vesicles from the clathrin shell. Four phosphosites of synaptojanin were quantified in this study that were not significantly regulated. However, these phosphosites were to some extent (2 fold) downregulated (S1160, T1048, S1053 and S1147). It is known that the enzymatic activity of synaptojanin is decreased by phosphorylation. Also, the previous report showed that binding of synaptojanin to endophilin1 is dependent on dephosphorylation of S1147 (Lee et al., 2004).

Besides the proteins involved in clathrin-mediated endocytosis, we have quantified phosphosites of proteins engaged in other modes of endocytosis. For example, Syndapin1 (S346) that functions in activity-dependent bulk endocytosis (Nguyen et al., 2014) and Endophilin A2 (S288) that play a role in membrane scission during clathrin-independent endocytosis (Renard et al., 2015).

\subsubsection{Synaptic vesicle and exocytosis related proteins}

Among synaptic vesicle proteins, synapsin 1 was the only protein with significantly regulated phosphosites (8 and 3 up and downregulated phosphosites, respectively) (Figure 3-15). Synapsin links synaptic vesicles to actin filaments and the physiological relevance of many synapsin phosphosites is already discovered. For example, it is known that phosphorylation at S603 and S566 is upregulated upon stimulation (in consistence with our observation in this study) and results in the conformational changes that decreases its affinity to actin filaments and synaptic vesicles (Benfenati et al., 1990). Consequently, more free vesicles are available for the docking and fusion to the membrane (Schiebler et al., 1986). It is also reported that S62, S67 and S549 are downregulated upon stimulation. However, in our dataset only S62 was significantly downregulated. It is known that phosphorylation of these 
sites also affects the structure of synapsin and decreases its affinity to actin but not synaptic vesicles (Jovanovic et al., 2000). It has been suggested that dephosphorylation at these sites creates a pool of the dephosphorylated synapsin that regulates recycling and reserve pool of synaptic vesicles (Jovanovic et al., 2001). In addition, it has been shown in a recent study that S551 is involved in the recruitment of synaptic vesicles to the readily releasable pool, and phosphorylation by CdK5 enhances the actin-binding of synapsin and consequently modulates the size of the readily releasable pool (Verstegen et al., 2014).

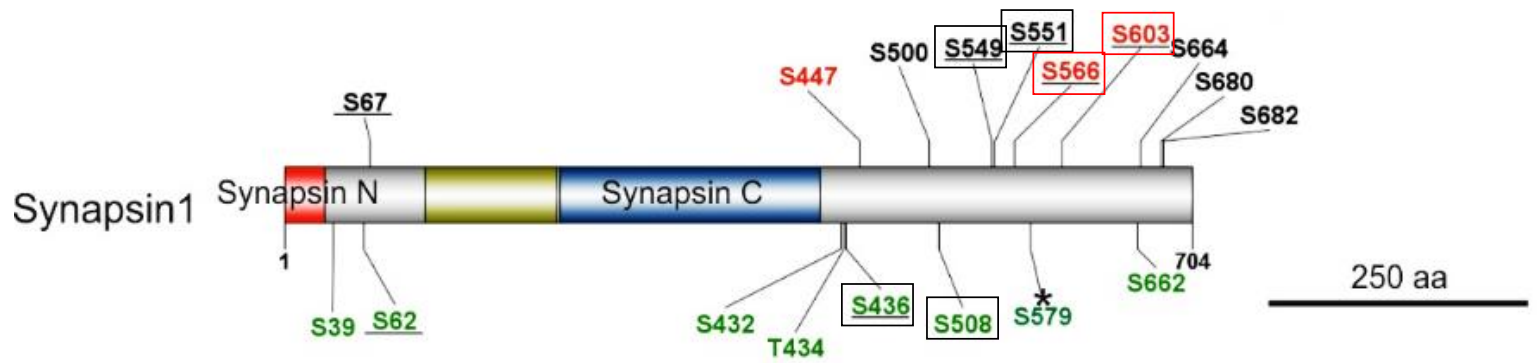

Figure 3-15: Regulation of synapsin1 phosphosites upon stimulation.

Quantified phosphosites of synapsin 1 are demonstrated. Up and downregulated synapsin1 phosphosites upon stimulation are represent in red/green, respectively. Phosphosites that are reported to be physiologically relevant are underlined. Phosphosites that are surrounded with the black/red box are the putative substrate of Calcineurin/CaMKII (see result chapter, part 3-3), adapted from (Kohansal-Nodehi et al., 2016).

Besides synapsin, we have quantified phosphosites in other synaptic vesicle proteins (Rab3a S188, S190, Sv2a S127, Sv2b S33, S36, synaptopodin S19, Syt1 T286, Syt12 S97, S99, Syt2 T128 and Syt3 587) that were not regulated upon stimulation. We have also quantified two phosphosites of SNARE proteins, Vamp2 S74 and S79 that were slightly upregulated. Both Syt1 and Vamp2 are known to be substrate of a variety of enzymes such as CaMKII and CK2 (Popoli, 1993, Davletov et al., 1993, Nielander et al., 1995), but the physiological relevance of the phosphorylation in vivo has not been studied.

In addition to synaptic vesicle proteins, phosphosites of some of the proteins involved in exocytosis were also regulated and that will be discussed in the following paragraphs. Complexins are known to regulate exocytosis via an interaction with the SNARE complex. It is known that complexin is phosphorylated at the C-terminus (S115) by casein kinase 2, and phosphorylation of this site enhances the binding of complexin to the SNARE complex (Shata et al., 2007). In our study, we have quantified another site of complexin1 (S93) that was upregulated upon stimulation. Investigation of the physiological relevance of S93 
phosphorylation can deepen our knowledge regarding the regulation of the neurotransmitter release by complexin.

We have quantified two phosphosites (S206 and S738) of the N-ethylmaleimide-sensitive fusion protein (NSF). NSF is an ATPase that disassembles the SNARE complex after fusion. We observed an upregulation of NSF at S206 upon stimulation. Phosphorylation of NSF at another site S237 was discovered as an inhibitory factor for the NSF activity (Matveeva et al., 2001).

Importantly, many phosphosites of proteins that are known to be involved in synaptic vesicle fusion were not regulated upon stimulation in our study: syntaxin1A (S14, T10, S59, and S64), SNAP-25 (S25) and Munc18 (S593, and S594). Of note, we have quantified 3 phosphosites of Munc18 that were slightly downregulated (S506, S507, and S509). Munc18 is known to be phosphorylated by PKC, CdK5, and ERK1/2 (Fletcher et al., 1999, Barclay et al., 2003, Schmitz et al., 2016).

\subsubsection{Cytoskeleton and associated proteins}

Both cytoskeleton filaments, actin, and microtubules are present in the nerve terminal. However, their localizationl is different. While actin filaments reach the active zone, the microtubules do not and are mostly present in the center of the nerve terminals (Doussau and Augustine, 2000). In this study, we have quantified 17 and 7 phosphosites as up and downregulated ones, respectively, that are associated with the cytoskeleton filaments and directly or indirectly modulate their structure. Some of these phosphosites will be described in the next paragraph.

Drebin is an actin-binding protein that stabilizes actin filaments and prevents them from depolymerization. Drebin (S241) was observed to be upregulated upon stimulation. It has been shown that phosphorylation (of another phosphosite, S142) via CdK5 modulate its binding to the actin filaments (Worth et al., 2013).

Another phosphosite that was shown to be downregulated is S17 of septin5. Septin5 is a GTPase and is known to regulate the exocytosis via interaction with syntaxin1. Phosphorylation of septin at S17 by CdK5 decreases its interaction with the syntaxin 1 (Taniguchi et al., 2007). 
Mysoin IIB (Myh10) is a motor protein that is known to be involved in the activity-evoked endocytosis of synaptic vesicles in the nerve terminal (Peng et al., 2012). We found two phosphosites of Mysoin IIB (S1952 and S1956) to be upregulated phosphosites upon stimulation. Interestingly, the previous study showed that a stretch of amino acids that contains the two mentioned serines is important for the dynamics of myosin (Juanes-Garcia et al., 2015).

MARCKS is an actin cross-linking protein that is known to be phosphorylated by PKC. MARCKS is also considered as the cross-bridge between actin filaments and the plasma membrane. We have found three phosphosites of MARCKS (S138, T143, and S163) to be upregulated upon stimulation. It is known that phosphorylation inhibits actin cross-linking by MARCKS (Nairn and Aderem, 1992).

\subsubsection{Ion Channels and transporters}

Presynaptic nerve terminals are rich in ion channels and transporters, and many of them are known to be regulated by phosphorylation. In this study, 103 phosphosites of channels and transporters were quantified in the $\mathrm{K}^{+}, \mathrm{Ca}^{2+}$ versus $\mathrm{K}^{+}$, EGTA comparison. Surprisingly, only 10 phosphosites out of 103 were detected as significantly changed. This suggests that channels and transporters are less targeted by kinase and phosphates upon stimulation in comparison to other groups of proteins, particularly the ones that are involved in SVrecycling. Below, I will discuss the phosphosites that were regulated.

We have quantified 5 phosphosites of $\mathrm{P} / \mathrm{Q}$-type calcium channel subunit alpha-1A and between them, only one (S1589) was observed to be significantly downregulated. The physiological relevance of these phosphosites is not known, but it has been reported that

phosphorylation of the P/Q-type calcium channel by CdK5 decreases the activity of the channel (Tomizawa et al., 2002).

FXYD domain-containing ion transport regulator 7 is another membrane protein that was found to be significantly downregulated at three phosphosites (S56, S58, S60). FXYD family is known to be associated with the $\mathrm{Na}$ /K-ATPase and decreases $\mathrm{Na}$ /K-ATPase affinity to $\mathrm{K}^{+}$ while does not impact the affinity towards $\mathrm{Na}^{+}$(Crambert et al., 2003). Na/K-ATPase is necessary to establish and maintain the high- $\mathrm{K}^{+}$and low- $\mathrm{Na}^{+}$concentration in the cytoplasm. 
It is conceivable that regulation of the phosphosite of FXYD affects the function of $\mathrm{Na} / \mathrm{K}$ ATPase in maintaining the balance between $\mathrm{K}^{+}$and low- $\mathrm{Na}^{+}$ions.

Two sites of sodium-dependent neutral amino acid transporter SLC6A17, S682 and S686, were observed to be downregulated upon stimulation. SLC6A17 is exclusively expressed in the central nervous system and transports proline, glycine, leucine and alanine across the plasma membrane in a sodium-dependent manner. There is no information available on the regulation of these transporter by phosphorylation.

We have observed three phosphosites of sodium/potassium/calcium exchanger 2 (Slc24a2), S41, S42 and S44, being upregulated upon stimulation. Slc24a2 is known to have a calcium channel activity, and in the retina it causes a rapid decrease of cytosolic calcium in the presence of light (Sharon et al., 2002). However, its role in the brain and precisely in the nerve terminal is not known. Since this protein resides in plasma membrane, it can be suggested that it functions as the calcium sensor and upon stimulation plays a role in the homeostasis of calcium concentration.

Moreover, we have quantified one phosphosite (S1103) of plasma membrane calciumtransporting ATPase 4 (Atp2b4) to be upregulated upon stimulation. This pump has a high

affinity for $\mathrm{Ca}^{2+}$ and is responsible for the maintaining of $\mathrm{Ca}^{2+}$ concentration at low level in the resting condition. Interestingly, the S1103 is known to be the substrate of PKC, and it increases the activity of the pump when phosphorylated. Therefore, it can be suggested that after stimulation of the synaptosomes, Atp2b4 gets phosphorylated at S1103 and consequently becomes more active in order to bring back $\mathrm{Ca}^{2+}$ concentration to the low level during the resting condition.

\subsubsection{Mapping Kinases and phosphatases responsible for the phosphorylation changes upon stimulation}

Next, we asked which kinases and phosphatases are responsible for the observed regulated phosphosites and how their activity is regulated upon stimulation. To answer this question, we used Motif-X software (Schwartz and Gygi, 2005) to investigate which sequence motifs are enriched among up and downregulated phosphosites. Our analysis revealed that the 'RxxS' sequence motif (where $S$ is the phosphorylation site and $x$ can be any amino acid) was overrepresented among significantly upregulated phosphopeptides in both $\mathrm{K}^{+}, \mathrm{Ca}^{2+}$ versus 
$\mathrm{K}^{+}$, EGTA (Fold increase: 7.36, Motif score: 16.00 ) and $\mathrm{K}^{+}, \mathrm{Ca}^{2+}$ versus EGTA (Fold increase: 5.98, Motif score: 12.93) comparisons (Figure 3-16 A). It is known that RxxS is the consensus motif of the CaMKII as well as PKA and conventional PKC isoforms. This result agrees well with the previous reports demonstrating an increased activity of these kinases upon depolarization of nerve terminals in the presence of calcium (Millan et al., 2003, Yamauchi, 2005).

A similar analysis was carried out for the phosphosites that are downregulated using Motif$\mathrm{X}$ software. Here, another sequence motif, 'SP' was enriched among the downregulated phosphosites in both $\mathrm{K}^{+}, \mathrm{Ca}^{2+}$ versus $\mathrm{K}^{+}$, EGTA (Fold increase: 8.03, Motif score: 16.00) and $\mathrm{K}^{+}, \mathrm{Ca}^{2+}$ versus EGTA (Fold increase: 8.21, Motif score: 16.00) comparisons (Figure 3-16 A). Phosphatases are not known to have the consensus motifs for their substrates. Nevertheless, 'SP' motif is known to be the consensus motif of proline-directed kinases such as Cdk5, GSK3 , and ERK1/2. This led us to conclude that activity of these kinases is downregulated upon stimulation. However, we have quantified two activating phosphosites in the activation loop of ERK1/2 (T183, Y185) as the upregulated phosphosites. That is why, ERK1/2 is less likely to be downregulated upon stimulation. Dephosphorylation of the phosphosites with 'SP' motif upon stimulation happens most likely due to one or both of the following reasons. First, CdK5 and GSK-3 are less active upon stimulation than during the resting condition. Second, phosphatases target 'SP' containing phosphosites more upon stimulation in comparison to the resting condition.

In addition to the motif analysis, we could detect further evidence for the modulation of phosphatase activity. For example, the phosphorylation of the regulatory subunit delta (LOC100909464) of protein phosphatase 2A (PP2A) at S567 was upregulated upon stimulation. This site is known to be phosphorylated by PKA, resulting in the increase of PP2A activity (Ahn et al., 2007). This suggests the higher activation of PP2A upon stimulation. Furthermore, upregulation in phosphorylation of Neurabin-2 S100, the regulatory protein of protein phosphatase 1 (PP1), was observed upon stimulation. It is known that phosphorylation of this site decreases the binding affinity of PP1 to the actin filaments and subsequently modulates its presynaptic localization, resulting in its diffusion from the nerve terminus (Colbran, 2004). 
Overall, the data suggest that upon the influx of $\mathrm{Ca}^{2+}$ to the nerve terminal subset of kinases and phosphatases such as PKA, PKC, CaMKII, PP2A, and PP2B are activated whereas the proline-directed kinases such as CdK5 and GSK-3 as well as PP1 are less active.

Next, in order to confirm whether the uncharacterized phosphosites containing RxxS motif are phosphorylated by one of the PKA, PKC, CaMKII kinases, we have checked the phosphorylation of five selected phosphosites of the active zone: RIM1 (S413 and S1141), bassoon (S2844), piccolo (S3626) and liprin- $\alpha 3$ (S75) in vitro by PKA, PKC and CaMKII kinases. For each site, a 13-mer peptide containing the desired phosphosite positioned in the middle was synthesized. The corresponding peptide with the alanine residue instead of serine (phosphosite) was used as negative control for each site. The result is shown in Figure 3-16 B. All of the peptides were phosphorylated at least by one of the kinases. For instance, bassoon S2844 was phosphorylated only by CaMKII. RIM1 (S413) was phosphorylated by PKA, which is consistent with the previous report (Lonart et al., 2003). RIM1 (S1141), bassoon (S2844) and piccolo (S3626) were phosphorylated by both CaMKII and PKA. Liprin$\alpha 3$ (S75) was the only phosphosite that was phosphorylated by all the three kinases. These results suggest that another regulatory mechanism should control the substrate-kinase relationship in vivo since each of the kinases showed selective phosphorylation of the substrates despite sharing the common core motif. 


\section{A}

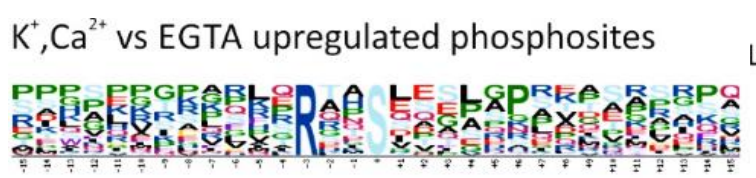

$\mathrm{K}^{+}, \mathrm{Ca}^{2+}$ vs $\mathrm{K}^{+}, \mathrm{EGTA}$ upregulated phosphosites

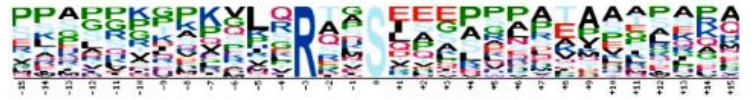

$\mathrm{K}^{+}, \mathrm{Ca}^{2+}$ vs EGTA downregulated phosphosites

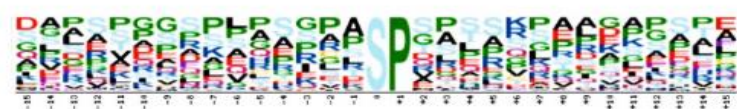

$\mathrm{K}^{+}, \mathrm{Ca}^{2+}$ vs $\mathrm{K}^{+}, \mathrm{EGTA}$ downregulated phosphosites

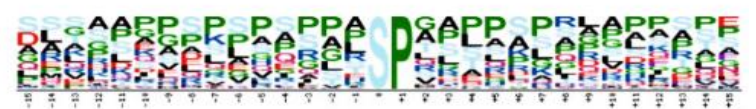

B

Liprin- $\alpha 3$

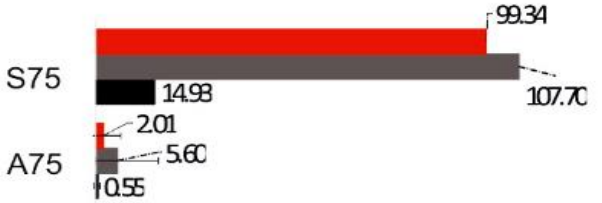

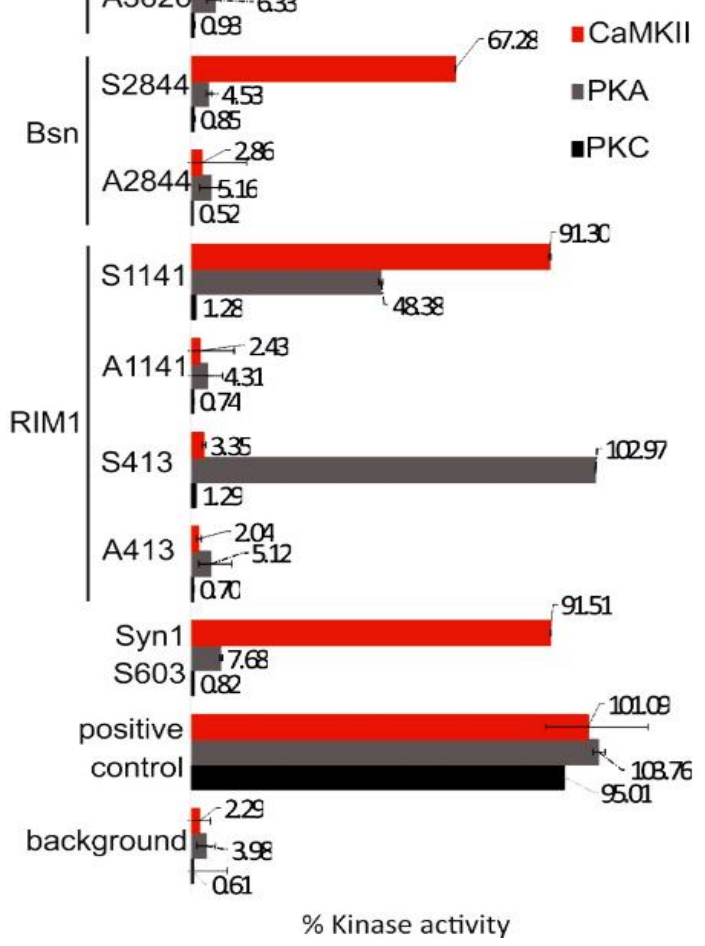

Figure 3-16: Motif analysis of the regulated phosphosites and experimental verification of the involved kinases.

(A) Sequence analysis of the up and down regulated phosphosites by Motif-X tool. The frequency of the residues surrounding the phosphosite (position 0) correlates with the size of the letter. For the up and downregulated phosphosites, 'RxxS' and 'SP' motifs were over-represented, respectively.

(B) Phosphorylation of five quantified phosphosites of the active zone proteins by PKA, PKC and CaMKII were tested by in vitro kinase assay. For each site, 13 amino acid long peptide was synthesized as a substrate in a way that the desired site was located in the middle of the peptide. As a negative control for each site the corresponding peptide with alanine substituting serine was used. For the positive control, autocamide-2, kemptide, and neurogranin were used as known peptide substrates of CaMKII, PKA, and PKC, respectively. The data represent mean values of three replicates, with the error bars indicating the range of values, adapted from (Kohansal-Nodehi et al., 2016) 


\subsection{Identification of the presynaptic substrates of CaMKII and Calcineurin (PP2B)}

From the data presented in the section 3-2, it is conceivable that CaMKII and Calcineurin are more active upon stimulation. The $\mathrm{Ca}^{2+}$-dependency of both enzymes has been extensively studied before (Miller and Kennedy, 1986, Sun et al., 2010). In addition, we described in the section 3.2.4 that phosphosites with the similar core motif can be targeted by more than one kinase in vitro. Therefore, in order to identify the substrate of the mentioned kinases in vivo upon stimulation, we have applied specific inhibitors of CaMKII (KN-93) and Calcineurin (Cyclosporine $\mathrm{A}$ ) and combined them with the phosphoproteomics workflow as it is depicted in the Figure 3-17 A, B.

To identify the Calcineurin substrates, synaptosomes were pre-incubated for 10 min with 10 $\mu \mathrm{M}$ of Cyclosporine A (Cyclo A) dissolved in DMSO, followed by stimulation with $\mathrm{KCl}$ (for the details see Material and Method section). Synaptosomes were incubated only with DMSO prior to the stimulation with $\mathrm{KCl}$ for the negative control. After 2 min stimulation both CycloA-treated synaptosomes and the control samples were dissolved in lysis buffer and equal amounts of them were acetone precipitated, digested and labeled by dimethyl labeling approach.

The labeled peptides of treated and control samples were mixed in the 1:1 ratio and fractionated by SCX. The first 8 fractions were collected, and phosphopeptides in each fraction were enriched by $\mathrm{TiO}_{2}$ method and analyzed by mass spectrometer. The phosphopeptides with the significant $\log 2$ ratio of control/CycloA less than 0 were considered as the putative substrates of Calcineurin, whereas the phosphopeptides with the significant log2 ratio control/CycloA more than 0 were the sites phosphorylation status of which was downregulated upon Calcineurin inhibition.

The same procedure was carried out to identify the CaMKII substrates, this time utilizing CaMKII inhibitor, KN-93. Here, the phosphosites with the significant log2 ratio of control/KN93 more than 0 were considered as the putative substrate of CaMKII, whereas the phosphopeptides with the significant log2 ratio control/KN-93 less than 0 were upregulated upon CaMKII inhibition (see Figure 3-17). 


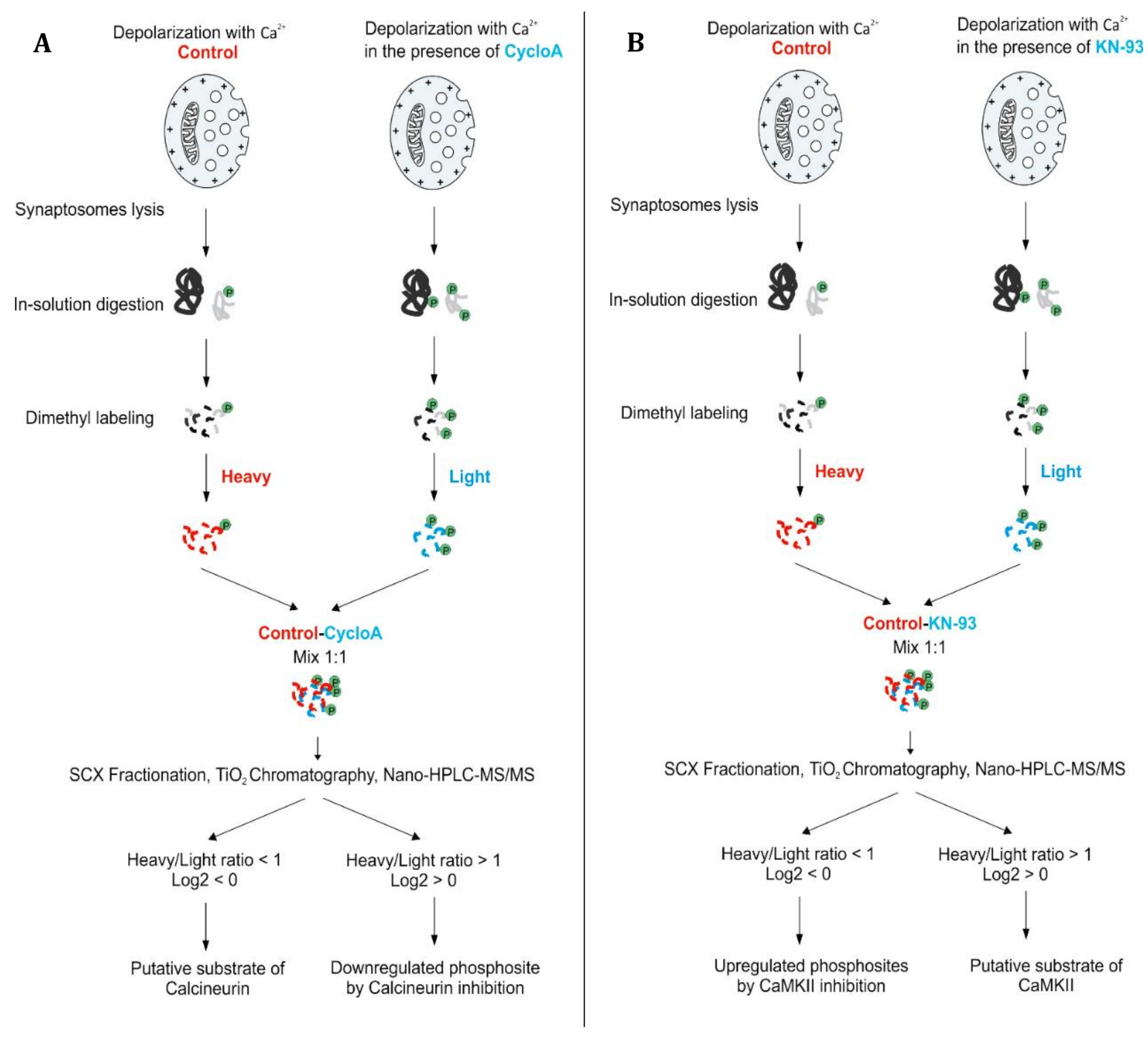

Figure 3-17: Experimental workflow for quantitative phosphoproteomics of synaptosomes treated with Calcineurin and CaMKII inhibitors.

Synaptosomes were treated either with the Calcineurin inhibitor, Cyclosporine A (CycloA) (A) or with the CaMKII inhibitor, KN-93 (B). Treated and non-treated synaptosomes (control) were stimulated in the presence of calcium by $\mathrm{KCl}$. The phosphoproteomics workflow is the same as mentioned in Figure 3-9. 


\subsubsection{Impact of Calcineurin inhibition on the exocytosis and phosphorylation status of the presynaptic proteins}

To access the effect of Calcineurin inhibition on the exocytosis, I have tested glutamate release of synaptosomes treated with $10 \mu \mathrm{M}$ Cyclosporine A (CycloA). As it is shown in Figure 3-18 A, CycloA-treated synaptosomes release glutamate even before $\mathrm{KCl}$ stimulation and the extent of this release was much higher than in the control samples. This phenomenon has been report in previous studies (Burley and Sihra, 2000, Sihra et al., 1995). In this reports, it was suggested that Calcineurin has an inhibitory effect of voltage-dependent calcium channels and upon Calcineurin inhibition both the basal and evoked $\mathrm{Ca}^{2+}$ influx into the nerve terminal are increased. Therefore, one explanation of the enhanced release of glutamate by CycloA-treated synaptosomes can be due to the higher activity of $\mathrm{Ca}^{2+}$ channels.

To ensure that the treatment with Cyclosporine A was efficient enough to reduce the activity of the Calcineurin, the phosphorylation status of dynamin1 S774 which is known to be the substrate of Calcineurin was investigated by phospho-specific antibody (Figure 3-18 B). The higher phosphorylation of the dynamin1 S774 was clearly observed in all replicates compared to control synaptosomes. Next, I have collected samples (four biological replicates) for MS analysis as it was mentioned in the previous section.

Figure 3-19 A demonstrates the distribution of the quantified phosphosites in pair-wise comparisons of CycloA-treated samples versus the control. To identify the significantly regulated phosphosites, I have used 'significant B' test (Perseus software). This test determines phosphosites with a significant outlier $\log 2$ ratio $\mathrm{H} / \mathrm{L}$ in the intensity-dependent manner relative to a total population of the phosphosites ( $p$-value $\leq 0.05$ ). All the phosphosites with a significant $\log 2$ ratio $\mathrm{H} / \mathrm{L}$ lower than 0 (red dots) were considered to be the putative substrates of Calcineurin. Note the position of the dynamin S774 as the known substrate of the Calcineurin as the upregulated site upon Calcineurin inhibition proves the high quality of the dataset. In addition, phosphorylation of more $50 \%$ of upregulated phosphosites upon stimulation $\left(\mathrm{K}^{+}, \mathrm{Ca}^{2+} \mathrm{vs}^{+}\right.$, EGTA) did not changed upon Calcineurin treatment (for instance synapsin S603, see the arrow in Figure 3-18), this suggests that the regulation of phosphosites by CycloA is specific and the basal release of glutamate by CycloAtreated synaptosomes is not due to deactivation of phosphorylation machinery upon CylcoA application. For the list of up and downregulated phosphosites upon Calcineurin inhibition 
see the Appendices section, Tables 6-4 and 6-5 (See Supplementary Table S4 for all quantified phosphosites in this experiment).

To have an overview of function of the putative substrates of Calcineurin, I have annotated them manually and categorized them into 11 groups. Surprisingly, it was revealed that the active zone proteins group have the highest number of phosphosites that are putative substrates of Calcineurin. This phosphosites are marked in the Figure 3-13 with the black boxes. These sites are distributed among the active zone proteins: bassoon (16 phosphosites), piccolo (11 phosphosites), RIM1 (3 phosphosites) Munc13 and liprin-a3 (each 1 phosphosite).

This group of proteins shares similarities with the proteins involved in clathrin-mediated endocytosis known as dephosphins. For instance, majority of their phosphosites undergo a cycle of phosphorylation/dephosphorylation in resting/stimulation status and both are the substrates of Calcineurin. Therefore, I named them "active zone dephosphins".

Cytoskeleton associated proteins group was the second group with the highest number of phosphosites as the Calcineurin substrate. This group contains mostly the microtubule associated proteins (Map1a, Map1b, Map7d1, Mapt), neurofilament polypeptides as well as proteins associated with the spectrin-actin network (beta-adductin and Ankyrin 2).

The other groups that were directly involved in the synaptic vesicle recycling were 'Exocytosis/SV proteins', 'channels/receptors' and 'Endocytosis'. Four phosphosites of synapsin1 (S508, S436, S549 and S551) were detected to be the substrates of Calcineurin. Dephosphorylation of synapsin at S549 and S551 by Calcineurin has been previously reported (Jovanovic et al., 2001). Also, Munc18 (S516) was detected as the substrate of Calcineurin. Phosphosites of voltage-dependent calcium channels (R-type subunit alpha-1E S15, N-type subunit alpha-1B S745, and P/Q-type subunit alpha-1A S1981) were also determined as Calcineurin substrates. The activity of calcium channels is known to be regulated by kinases such as GSK-3 (Zhu et al., 2010) and Cdk5 (Su et al., 2012). While it has been shown that Calcineurin enhances the activity of L-type calcium channels (Norris et al., 2002), no phosphosite has been detected to be a substrate of Calcineurin in our dataset. Dephosphorylation of voltage-dependent calcium channels can be the explanation for the stimulus independent release of glutamate from CycloA-treated synaptosomes. 

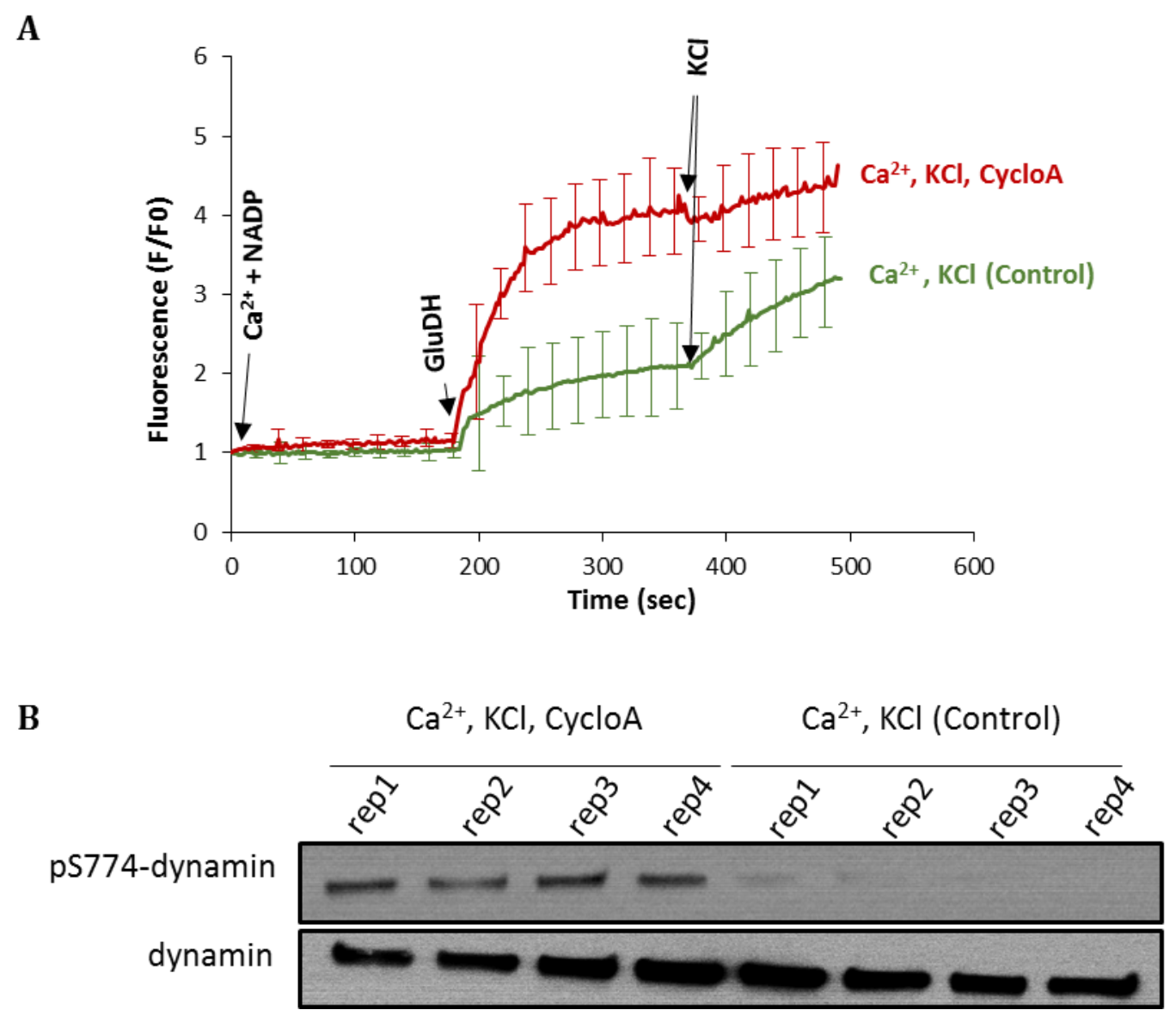

Figure 3-18: Glutamate release assay and immunoblot analysis of synaptosomes treated with Cyclosporine A.

(A) Glutamate release was measured for the Cyclosporine A treated $\left(\mathrm{Ca}^{2+}, \mathrm{KCl}, \mathrm{CycloA}\right)$ and non-treated $\left(\mathrm{Ca}^{2+}\right.$, $\mathrm{KCl})$ synaptosomes by $\mathrm{KCl}$ stimulation in the presence of calcium. Treated synaptosome released glutamate even before stimulation and with the much higher extent compared to the control. Data are represented as a mean value of three replicates, with the error bars indicating the range of values.

(B) Immunoblot analysis of dynamin S774 phosphorylation as the known substrate of Calcineurin. Synaptosomes were collected 2 min after stimulation for immunoblot analysis with phospho-specific antibody against dynamin pS774. Using the Calcineurin inhibitor almost abolished dephosphorylation of dynamin pS774 upon stimulation in the presence of calcium. For the loading control antibody against dynamin that is not sensitive to the phosphorylation was used.

In line with the previous report (Cousin and Robinson, 2001), proteins involved in endocytosis of synaptic vesicles were also detected as the Calcineurin substrates. Dynamin1 (S774 and 778), AP180 (S300), Endophilin-A2 (S288) and tyrosine-protein phosphatase auxilin (S568) were detected as putative substrates of Calcineurin. However, phosphosites of the amphiphysins1 and 2, epsin, eps15 and other members of the dephosphins were not detected as substrates of the Calcineurin. 
A

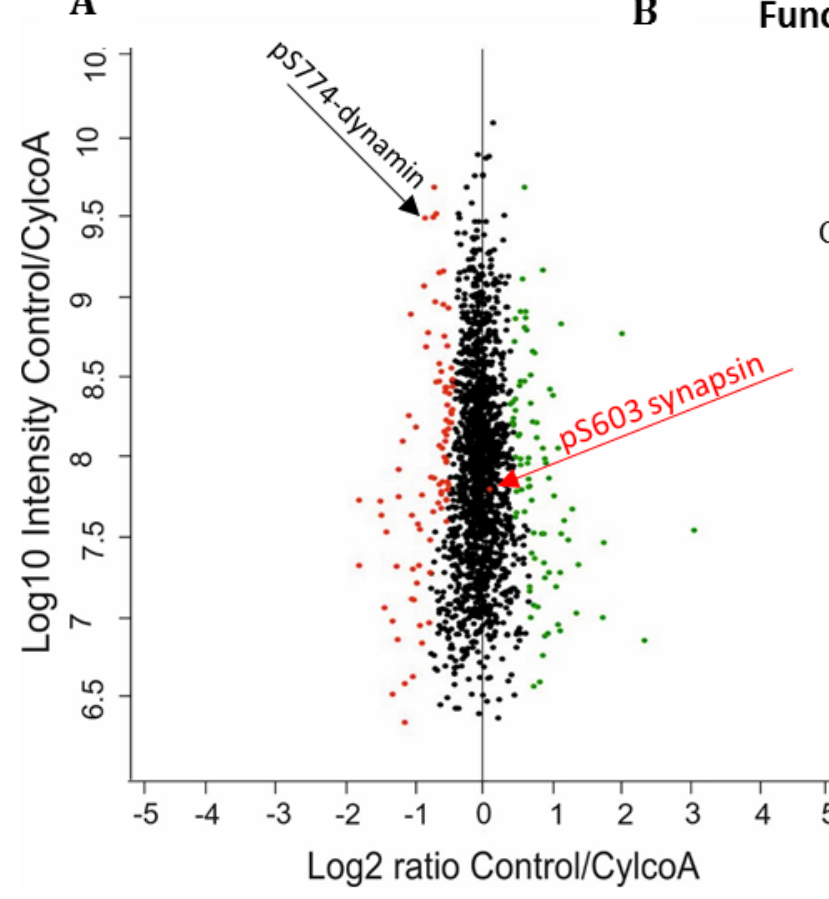

\section{B Functional/Localization distribution of Putative} substrates of Calcineurin

- Putative substrates of Calcineurin

- Phosphosites that are downregulated by Calcineurin inhibition

- $\quad$ Not affected phosphosites by Calcineurin inhibition

Figure 3-19: Overview of the quantified phosphosites of synaptosomes treated with Cyclosporine A.

(A) Distribution of quantified phosphosites from pair-wise comparisons of treated synaptosomes with Cyclosporine A versus untreated synaptosomes (control). Stable-isotope dimethyl labeling was used for the quantification. To determine the significantly regulated phosphosites the two-tailed 'Significant B' test (p-value $\leq 0.05$ ) of the Perseus module was used (See Material and Method). The red dots correspond to the phosphosites that were significantly upregulated in the treated synaptosomes upon stimulation and are considered to be the putative substrate of Calcineurin. The green dots are the sites that were downregulated by Calcineurin inhibition. Black dots represent the sites that were not significantly affected by Calcineurin inhibition. The position of dynamin 1 S774 and synapsin1 S603 are marked with the arrow as the known substrate of Calcineurin and CaMKII, respectively.

(B) Proteins containing phosphosites as the putative substrates of Calcineurin were manually categorized based on their function and localization into 11 groups according to the previous publication (Boyken et al., 2013). Proteins with other function/localization than listed or uncharacterized proteins were grouped into 'other' category. Active zone and Cytoskeleton categories were observed as groups containing the highest number of phosphosites as the putative substrates of Calcineurin.

Phosphosites of CaMKII (CaMKIIa S279, CaMKIIb S397, T400, and T401) were dephosphorylated by Calcineurin. Interestingly, the phosphosites of CaMKIIb S397, T400 and T401 are not involved in the activity regulation of CaMKII. These phosphosites are located in the association domain and actin binding domains of CaMKII and known to regulate the localization of CaMKII (Kim et al., 2015). 
Next, I used Motif-X to test whether there is a specific motif enriched among the putative substrates of Calcineurin. As it is shown in the Figure 3-20 A, the motif 'SP' (Fold increase: 31.75, Motif score: 21.29) and 'SxxxSP' (Fold increase: 11.37, Motif score: 16.00) were overrepresented among the putative substrates of Calcineurin. This result agrees well with the previous experiment described in the section 3.2.4 indicating dephosphorylation of phosphosites with 'SP' motif upon stimulation. The SxxxSP motif is known to be the consensus motif of GSK-3 $\beta$ (Roach, 1991). Interestingly, around $40 \%$ and $25 \%$ of the Calcineurin substrates possess 'SP' and 'SxxxSP' motif, respectively. Furthermore, the substantial percentage of Calcineurin substrates in the active zone has the same motifs (almost 48\% for 'SP' and 20\% for 'SxxxSP', Figure 3-20 B). From this result, it can be suggested that GSK-3 $\beta$ and other proline-directed kinases, especially CdK5, have a significant role in the phosphorylation of active zone proteins in the resting condition.

A

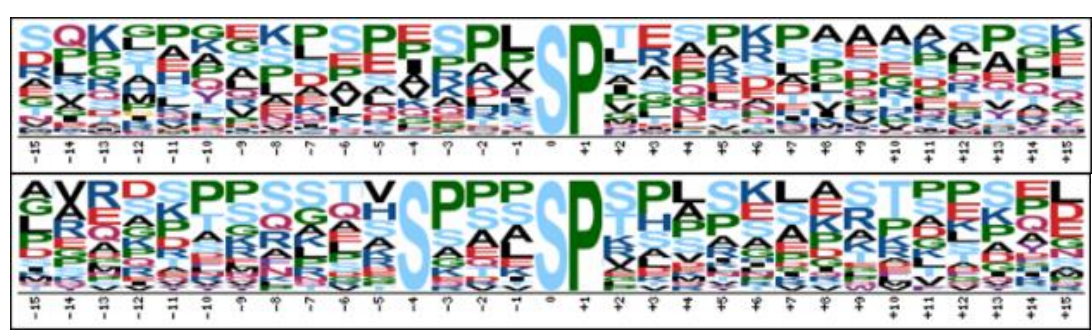

B

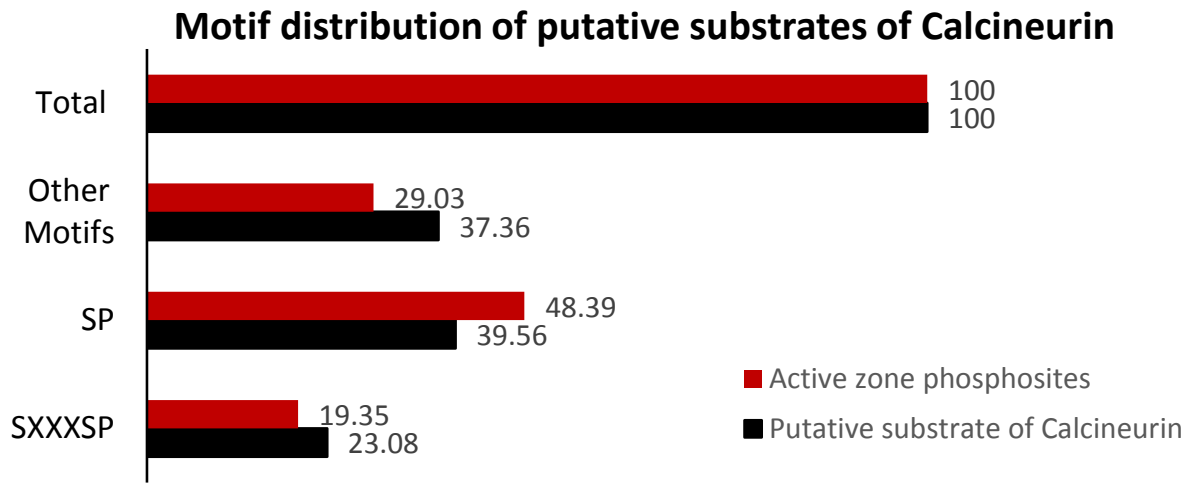

Figure 3-20: Motif analysis of the putative substrates of Calcineurin.

(A) The sequence of phosphopeptides containing putative substrates of Calcineurin were analyzed by Motif-X tool to identify the overrepresented sequence motifs. The frequency of the residues surrounding the phosphosite (position 0) correlates with the size of the letter. Motif 'SP' and 'SxxxSP' were observed to be overrepresented.

(B) Distribution of overrepresented motifs among all putative substrates of Calcineurin and active zone phosphosites. Almost 50\% and 20\% of the active zone sites have the 'SP' and 'SxxxSP' motifs, respectively. 


\section{Phosphosites downregulated by Calcineurin inhibition}

Upregulation of the phosphosites (putative substrates of Calcineurin) was not the only consequence of Calcineurin inhibition. Downregulation of other sets of phosphosites was also observed. One explanation for this phenomenon is the indirect effect of Calcineurin on the other kinases and phosphatases. In fact, I have observed 6 phosphosites in the regulatory subunits of phosphatases and 10 phosphosites of kinases among the downregulated phosphosites upon Calcineurin inhibition. Except for ERK kinases and the Calcipressin-1, no published data has described the regulation of other phosphosites. Below, I have discussed the role ERK kinases and the Calcipressin-1 phosphosites in more detail.

The two phosphosites of ERK1 (T183, Y185) were downregulated upon Calcineurin inhibition. It is known that these sites are located in the activation loop of the ERK kinase and their phosphorylation increases the activity of ERK. In contrast, dephosphorylation of these sites decreases the enzymatic activity of ERK1, demonstrating that ERK1 and the Calcineurin are co-deactivated.

Calcipressin-1 (RCAN1) is a known regulator protein of Calcineurin. In our dataset, two phosphosites of Calcipressin-1 (S108 and S112) were observed to be downregulated upon Calcineurin inhibition. It is known that these phosphosites are phosphorylated by GSK-3 $\beta$ and ERK kinases, respectively, and phosphorylation of these two sites has a dual effect on the regulation of Calcineurin. While it increases the inhibitory effect of Calcipressin-1 on Calcineurin, it decreases its own half-life (Genesca et al., 2003, Ma et al., 2012). Therefore, it is conceivable that Calcineurin auto-regulates itself via Calcipressin-1 dephosphorylation.

Using Motif-X, I have observed two motifs, 'SP' (Fold increase: 3.54, Motif score: 5.16) and 'SxxE' (Fold increase: 4.29, Motif score: 5.62), as the overrepresented motifs (Figure 3-21) among the downregulated phosphosites upon Calcineurin inhibition. While the 'SP' motif is the core target-site of proline-directed kinases, the 'SxxE' is the consensus motif of the casein kinase2 (CK2). Since downregulation of two activating phosphosites of ERK kinase upon Calcineurin inhibition was observed (see above), it is probable that enrichment of 'SP' motif among the downregulated sites is the consequence of ERK inhibition.

Regulation of CK2 by phosphorylation has been previously reported. Interestingly, it has been observed that incubation of CK2 with a variety of phosphatases increases its activity (Agostinis et al., 1987). Therefore, based on this study and overrepresentation of SxxE motif 
among the downregulated phosphosites upon Calcineurin inhibition, it can be concluded that CK2 activity is co-regulated by Calcineurin.

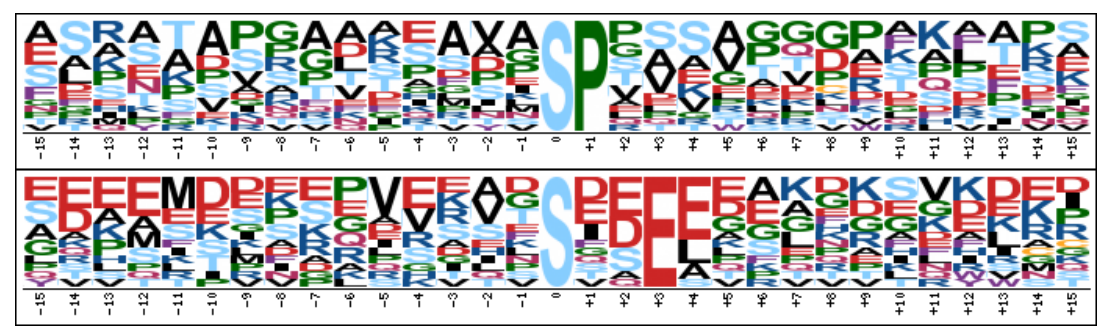

Figure 3-21: Motif analysis of the downregulated phosphosites by Calcineurin inhibition.

The sequences of the downregulated phosphopeptides upon Calcineurin inhibition were analyzed by Motif-X tool to identify the overrepresented motifs. The frequency of the residues surrounding of the phosphosite (position 0) correlats to the size of the letter. 'SP' and 'SXXE' motifs were observed to be overrepresented in this dataset.

\subsubsection{Impact of CaMKII inhibition on the exocytosis and phosphorylation status of the presynaptic proteins}

The postsynaptic role of CaMKII in synaptic plasticity and memory has been well studied (Lisman et al., 2002). However, our knowledge regarding the presynaptic role of CaMKII in neurotransmission is limited. Here, I have used the CaMKII inhibitor (KN-93) in combination with the phosphoproteomics workflow to identify the phosphosites that are affected by CaMKII upon stimulation (see Figure 3-17). First, I have tested the release of glutamate in the synaptosomes treated with the $10 \mu \mathrm{M} \mathrm{KN}-93$. As it is shown in the Figure 3-22 A, treatment of synaptosomes with KN-93 decreased the release of glutamate compared to control synaptosomes. This result is consistent with previous reports (Ninan and Arancio, 2004, Llinas et al., 1991, Llinas et al., 1985). In the next step, I have used a phospho-specific antibody to check the status of synapsin S603 (the known substrate of CaMKII) in the treated and control synaptosomes after stimulation with $\mathrm{KCl}$, to ensure that $\mathrm{KN}-93$ treatment was sufficient to decrease the activity of CaMKII (Figure 3-22 B). Treatment with KN-93 decreased the phosphorylation of synapsin S603 in all the three biological replicates. 

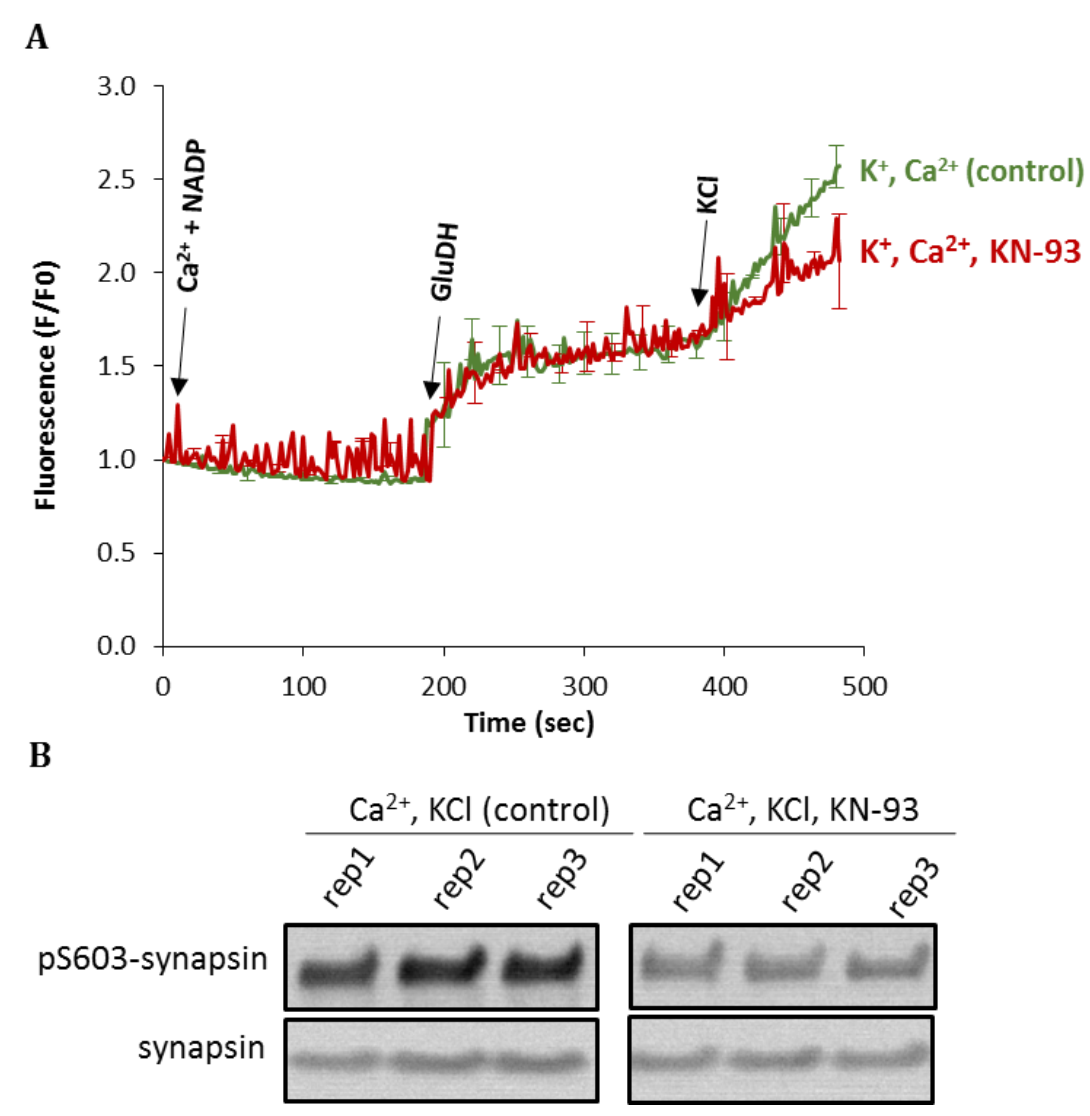

Figure 3-22: Glutamate release assay and immunoblot analysis of synaptosomes treated with KN-93. (A) Glutamate release was measured for the $\mathrm{KN}-93$ treated $\left(\mathrm{Ca}^{2+}, \mathrm{KCl}, \mathrm{KN}-93\right)$ and not treated $\left(\mathrm{Ca}^{2+}, \mathrm{KCl}\right)$ synaptosomes upon $\mathrm{KCl}$ stimulation in the presence of calcium. The release of glutamate by the treated synaptosomes with KN-93 was lower than by the control synaptosomes. Data represent a mean of three replicates, with the bars indicating the range of values.

(B) Immunoblot analysis of phospho-synapsin S603 as the known substrate of CaMKII. Synaptosomes were collected 2 min after stimulation for immunoblot analysis with the phospho-specific antibody against synapsin pS603. Application of the CaMKII inhibitor decreased the phosphorylation of synapsin pS603 upon stimulation in the presence of calcium in all replicates. Antibody against synapsin that is not sensitive to the phosphorylation was used as a loading control.

Figure 3-23 A shows the distribution of the quantified phosphosites in the pair-wise comparisons of KN-93-treated samples to the control. A shift towards the positive values could be observed in the graph. Phosphosites with the dimethyl ratio higher than $1(\log 2>$ 0) represent the phosphosites that are downregulated upon CaMKII inhibition. Due to the observed shift toward positive values, I have defined the significantly downregulated sites upon CaMKII inhibition based on the two parameters. First, phosphosites with the outlier $\log 2$ ratio $\mathrm{H} / \mathrm{L}$ relative to a total population of the phosphosites based on the one-tailed 'significant B' test ( $p$-value $\leq 0.05$, Perseus software, red dots). Second, all the phosphosites with the log2 ratio higher than 1 (two fold downregulation, moss dots). Note the position of 
the synapsin1 S566 as known substrate of the CaMKII among the downregulated phosphosites.

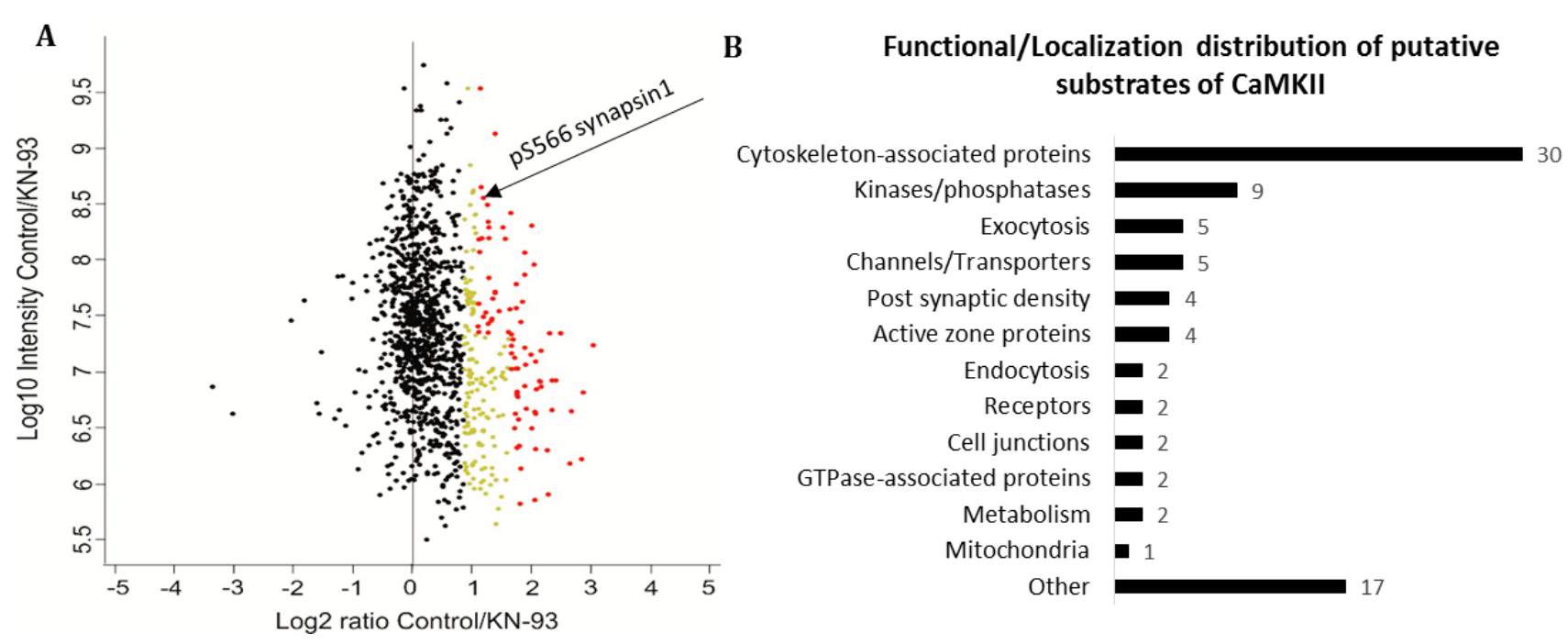

Figure 3-23: Overview of the quantified phosphosites of synaptosomes treated with KN-93.

(A) Distribution of quantified phosphosites from the pair-wise comparisons of treated synaptosomes with KN93 versus untreated synaptosomes (control). Stable isotope dimethyl labeling was used for the quantification. A shift towards the right was observed in the distribution of phosphosites, showing that many phosphosites were directly or indirectly downregulated by the CaMKII inhibition. To determine significantly regulated phosphosites, two methods were used. First, one-tailed 'Significant B' test (p-value $\leq 0.05$ ) of the Perseus module (See Material and Method) similar to the previous experiment (red dots). Second, all the sites that are downregulated at least by 2 fold upon CaMKII inhibition (Log2=1) were considered as the downregulated phosphosites (moss dots). The position of the synapsin1 S566 is shown as the known substrate of CaMKII.

(B) Significantly downregulated sites (determined by Significant B test, red dots in A) were categorized based on their function and localization into 11 groups according to a previous publication (Boyken et al., 2013). Proteins with function/localization other than listed or uncharacterized proteins were grouped into 'other' category. Here, we observed Cytoskeleton and Kinase/phosphatase categories as the groups containing the highest number of CaMKII substrates.

Sequence analysis of the significantly downregulated sites (determined by 'Significant B' test, red dots in the Figure 3-23 A) revealed 'SP' (Fold increase: 5.19, Motif score: 12.07) and 'SXE' (Fold increase: 5.62, Motif score: 4.62) motifs to be enriched (Figure 3-24 A). Surprisingly, the consensus motif of the CaMKII (R/KxxS) was enriched. Next, I repeated motif analysis among all the phosphosites that were at least two fold downregulated upon CaMKII inhibition (moss and red dots in Figure 3-23 A). Here, besides consensus motif of prolinedirected kinases (SP), casein kinase 2 motifs (SxEE (Fold increase: 17.08, Motif score: 17.04) and SxD (Fold increase: 4.75, Motif score: 5.61)), the CaMKII motifs (KxxS, Fold increase: 4.74, Motif score: 8.79 and RxxS, Fold increase: 3.9, Motif score: 8.18) were also enriched (Figure 3-24 B). 
Interestingly, comparison of downregulated phosphosites by CaMKII inhibition with regulated phosphosites upon stimulation $\left(\mathrm{K}^{+}, \mathrm{Ca}^{2+}\right.$ vs $\left.\mathrm{K}^{+}, \mathrm{EGTA}\right)$ revealed that most of the downregulated phosphosites upon CaMKII inhibition (83\%) are not regulated upon stimulation (Figure 3-24 C). Almost $16 \%$ of sites are upregulated, containing the phosphosites that are known to be the CaMKII substrates, for instance synapsin S603 and S566, CaMKII T287 and S279 and bassoon S2844. Importantly, only 1\% of phosphosites are among the downregulated phosphosites upon stimulation. This proves that the consequence of CaMKII inhibition on phosphosites is specific and has a minimum effect on phosphosites that are downregulated upon stimulation.

Figure 3-24 D shows the distribution of the enriched motifs among the downregulated sites. Surprisingly, the substrates of proline-directed kinases were affected more (30\%) than the CaMKII substrates (20\%). We observed that the CK2 substrates were also affected (12\%). One potential explanation of this finding is that CaMKII regulates the activity of other kinases and phosphatases directly or indirectly via phosphorylation. To answer this question, I have manually annotated significantly regulated sites (Figure 3-23 A, red dots) and categorized them into 13 groups. Proteins that are associated with the cytoskeleton appeared to be the group with the highest number of the regulated phosphosites. As it was expected, 'Kinase and Phosphatases' group was the second most regulated group. Phosphosites that belong to 'Exocytosis', 'Channel and Transporters' and 'Active zone' proteins were the following groups with the highest number of regulated phosphosites. Below I have discussed some of these phosphosites in more detail. For the complete list of the downregulated sites upon CaMKII inhibition see Table 6-6 (All the quantified phosphosites in this experiment is listed in Supplementary Table S5). 
A

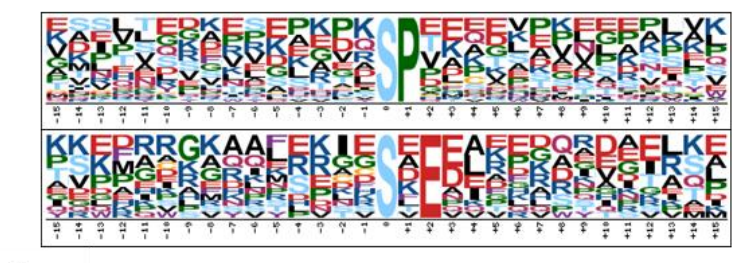

B

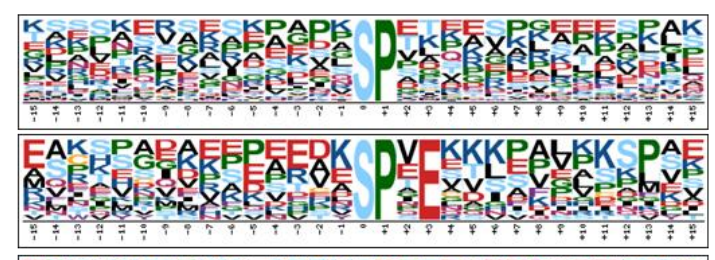

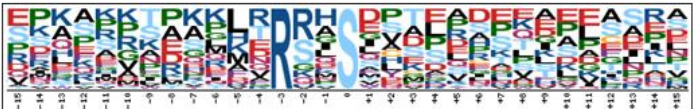
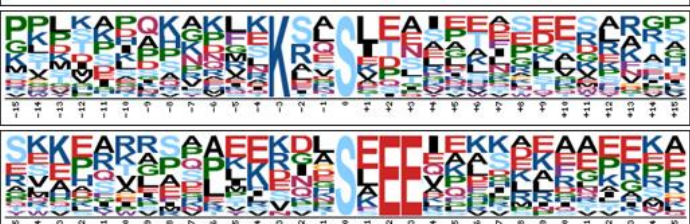

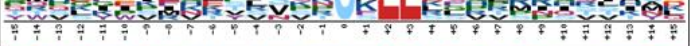

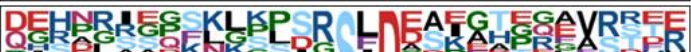

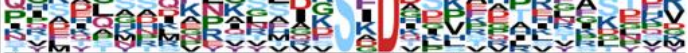

C $\quad \%$ of downregulated sites by CaMKII inhibtion upon stimulation

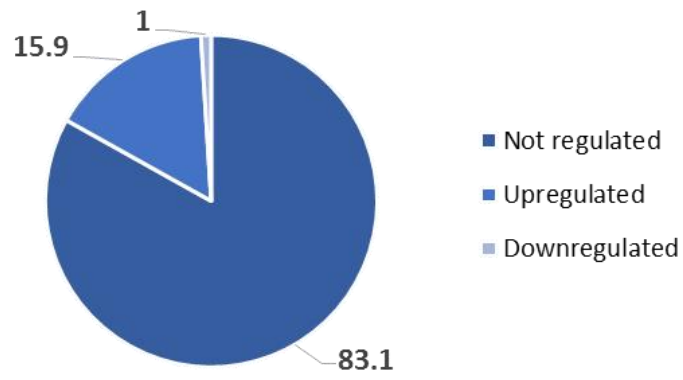

D Motif distribution of putative substrates of CaMKII

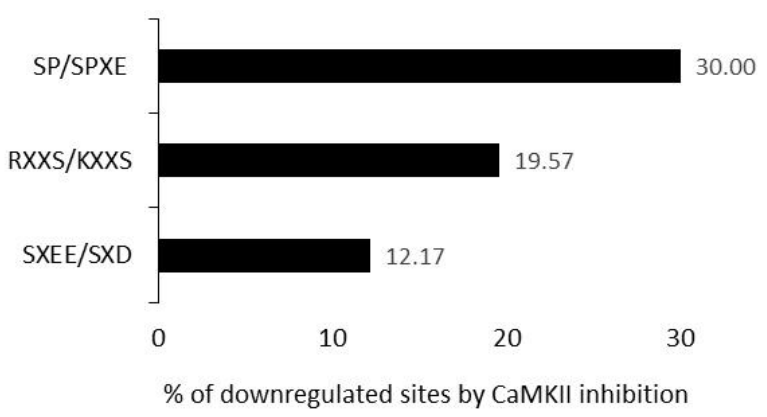

Figure 3-24: Analysis of the downregulated phosphosites by CaMKII inhibition.

(A) Sequence of the phosphopeptides determined to be significantly downregulated by 'significant B test' upon CaMKII inhibition were analyzed by Motif-X tool to identify the overrepresented sequences. Motif 'SP' and 'SxE' were the most frequent ones. The frequency of the residues surrounding of the phosphosite (position 0 ) correlates with the size of the letter.

(B) The sequence of the phosphopeptides downregulated at least two fold upon CaMKII inhibition were analyzed by Motif-X tool to identify the overrepresented sequences. Here, motif 'SP', 'SPxE', 'SxEE', 'SxD' as well as the known consensus motif of CaMKII substrate, 'K/RxxS' were observed to be overrepresented. The frequency of the residues surrounding the phosphosite (position 0 ) correlates with the size of the letter.

(C) Regulation analysis of downregulated phosphosites by CaMKII inhibition upon stimulation $\left(\mathrm{K}^{+}, \mathrm{Ca}^{2+}\right.$ vs $\mathrm{K}^{+}$, EGTA). CaMKII inhibition has the most and least effect on not regulated and downregulated phosphosites upon stimulation, respectively.

(D) Distribution of the overrepresented motifs among the downregulated phosphosites. Almost $20 \%$ of the downregulated phosphosites contain the known consensus motif of CaMKII substrate (K/RxxS).

\section{Cytoskeleton-associated phosphosites}

Alpha-adducin is an actin-binding protein and is known to have a role in the regulation of actin rings in the axon (Leite et al., 2016). In our dataset, S355, S600, and S610 of Alphaadducin were observed to be downregulated upon CaMKII inhibition. It is known that S355 is phosphorylated by CdK1 and ERK2 enabling alpha-adducin to bind myosin-X (Myo10)(Chan et al., 2014). 
Stathmin is a tubulin binding protein that inhibits the polymerization of microtubules. In our dataset, downregulation of stathmin S16 was observed. In fact, this site is known to be the CaMKII substrate (le Gouvello et al., 1998). Its phosphorylation weakens the binding of stathmin to the tubulin (Di Paolo et al., 1997).

Phosphosites of neurofilament polypeptides, medium polypeptide (S774, S872) and heavy polypeptide (S712, S721) were also observed as the downregulated site upon CaMKII inhibition. It is known that neurofilaments contain a repetitive sequence of KSP that is a target of proline-directed kinases such as ERK, GSK-3 and CdK5 (Holmgren et al., 2012). Neurofilament phosphorylation plays a critical role in plasticity, stability and the width of neurofilaments (Pant and Veeranna, 1995, Kesavapany et al., 2003). Indeed, three phosphosites out of the four downregulated sites in our dataset, have the 'SP' motif.

Moreover, in our dataset, microtubule-associated protein tau has been also observed to have downregulated sites upon CaMKII inhibition (S573, S150, and S33). These sites are known to be phosphorylated and dephosphorylated by GSK-3 $\beta$ and PP2A, respectively (Qian et al., 2010). It is known that tau aggregation is involved in a variety of neurodegenerative diseases and it is more susceptible to aggregation in the hyperphosphorylated state (Wang et al., 2013). However, there is no available information regarding the role of tau phosphorylation in the neurotransmitter release.

In addition, two phosphosites of Septin5 (T13, S234) that are involved in the regulation of neurotransmitter release (Amin et al., 2008) were downregulated upon CaMKII inhibition.

\section{Kinases and phosphatases}

As it was mentioned above, it is revealed that component of kinases and phosphatases group was downregulated significantly upon CaMKII inhibition. Some of these phosphosites are located in the activation loop of these enzymes and their phosphorylation is known to be involved in the regulation of enzymatic activity. Overall, regulation of the phosphosites of kinases and phosphatases together with the enrichment of consensus motifs of prolinedirected kinases and CK2 among the downregulated sites upon CaMKII inhibition would suggest that these kinases and phosphatases are regulated by CaMKII (directly or indirectly). Below I have discussed these downregulated sites in more detail. 
Four phosphosites of CaMKII were detected to be downregulated upon phosphorylation. CaMKIIb T287 is known to be the autophosphorylation site, which when phosphorylated makes the activity of the CaMKII independent from $\mathrm{Ca}^{2+}$ concentration (Miller and Kennedy, 1986). Another identified downregulated phosphosite was S279 of CaMKIIa. This site is also known as an autophosphorylation site that decreases the enzymatic activity of CaMKII (Lengyel et al., 2000). Three other phosphosites of CaMKIIb were also identified as downregulated sites (S276, S280, and S395). These sites are not known to be involved in the phosphorylation activity of CaMKII but they have a role in actin binding and association of CaMKII subunits (Kim et al., 2015).

Phosphosites of protein kinase A were also identified as the downregulated sites, one site in the catalytic subunit (Prkaca, T198) and one site in the regulatory subunit (Prkar2a, S97). Phosphorylation of T198 in catalytic subunit is known to be necessary for the maximum activity of the PKA (Adams et al., 1995) and it is either autophosphorylated or phosphorylated by PDK-1 (Moore et al., 2002). Downregulation of this site upon inhibition of CaMKII suggests that CaMKII and PKA are co-deactivated. The phosphosite in regulatory subunit $\mathrm{S} 97$ is known to be the substrate of CK2 but there is no evidence that phosphorylation of this site affects the activity of PKA (Carmichael et al., 1982).

Several phosphosites of protein kinase $\mathrm{C}$ were also identified as downregulated sites upon CaMKII inhibition. One of them is PKC $\alpha$ T497 that is located in activation loop of the kinase and is known to be phosphorylated by PDK-1 resulting in activation of PKC (Dutil et al., 1998). The other phosphosite is PKC $\beta$ S642. This site is known to be the autophosphorylation site and regulates binding of PKC to phorbol esters that are necessary for its activation (Flint et al., 1990). In addition, two phosphosites of $\mathrm{PKC} \varepsilon$, S346 and S350, were identified as the downregulated sites. These sites are known to be phosphorylated by GSK-3 and MAPK, respectively and have a role in binding of PKC to 14-3-3 adaptor protein (Saurin et al., 2008). MARK1, T215 is another phosphosite that was identified as downregulated site upon CaMKII inhibition. Mark is known to phosphorylate microtubules associated proteins such as tau, MAP2, and MAP4 resulting in the disassociation of them from microtubules (Manning et al., 2002). MARK1 is phosphorylated at T215 by LKB that enhances its activity (Lizcano et al., 2004). 
Beside kinases, phosphosites that are associated with the phosphatases were also downregulated upon CaMKII inhibition. One example is PP1 regulatory subunit 1B (DARPP32) that is known to inhibit the phosphatase activity of PP1 only when it is phosphorylated at T34 by PKA. In our dataset, we have quantified another phosphosite of DARPP-32, S102 as the downregulated site. This site is known to be phosphorylated by CK2 and upon phosphorylation facilitates the phosphorylation of T34 by PKA (Girault et al., 1989). Overall, this suggests that when CaMKII is less active the inhibitory effect of DARPP-32 on the PP1 is decreased indirectly through the lowered activation of CK2 and PKA.

Moreover, two more phosphosites related to the phosphatases were identified (Protein phosphatase 1H (S220) and Calcineurin catalytic subunit (S479)). To the best of our knowledge no information is available regarding the regulation of these phosphatases via phosphorylation of the mentioned sites.

\section{Exocytosis Related proteins}

Synapsin is the best-studied phosphoprotein in the nerve terminal. Here, besides synapsin1 S603 that was shown to be downregulated upon CaMKII inhibition by immunoblotting, synapsin1 S556 was also identified as the downregulated site. Both of these sites are known to be a substrate of CaMKII with their phosphorylation decreasing the binding of synapsin to actin filaments (Jovanovic et al., 2001).

Complexin1 at S93 was identified as a downregulated site upon CaMKII inhibition. Interestingly, this site was detected in the previous experiment as the upregulated phosphosite upon stimulation (See section 3.2.3.3).

Among the active zone proteins, two phosphosites of RIM1 (S991 and S1277) and two phosphosites of bassoon (S2844 and S2850) were downregulated. RIM1 S991 and bassoon S2844 were quantified as the upregulated phosphosites upon stimulation of synaptosomes (See section 3.2.3.1) and S2844 was determined as the substrate of CaMKII in the previous experiments by in vitro kinase assay (See Figure 3.16 B). Overall, these observations are consistent with the former studies as well as our previous experiments in which phosphorylation of CaMKII substrates was upregulated upon stimulation of synaptosomes. 


\section{Discussion}

In this thesis, a combination of MS-based phosphoproteomics and subcellular fractionation methods has been employed to investigate the regulation of phosphorylation changes of presynaptic proteins upon stimulation. These data provided detailed and valuable information regarding the phosphoproteome of presynapse and its regulation. Furthermore, through data analysis, in vitro kinase assays and application of pharmacological inhibitors of kinases and phosphatases, I was able to show that kinases and phosphatases in the presynapse coordinate tightly to facilitate and regulate the exo- and endocytosis upon stimulation. In this section, significance of my results in the context of synaptic vesicle recycling as well as the advantages and limitations of the presented approach are discussed.

\subsection{Activity-dependent phosphorylation changes of nerve terminal proteins}

\subsection{1 $\mathrm{Ca}^{2+}$ ions are the main regulator of phosphorylation upon stimulation}

As outlined in the introduction, at chemical synapse, neurons communicate with each other via two mechanisms known as fast and slow synaptic transmission. Fast synaptic transmission happens within milliseconds and is triggered by the arrival of action potential to the nerve terminal, resulting in the release of neurotransmitters. In contrast, slow synaptic transmission occurs over the course of hundreds of milliseconds to several minutes. Detection of many phosphoproteins, kinases, and phosphatases in the nerve terminal supported the idea that phosphorylation might be involved in the regulation of synaptic transmission. Relatively slow enzymatic reactions catalyzed by kinases/phosphatases makes it less likely to regulate the fast synaptic transmission. On the other hand, several studies have established that these enzymes regulate the slow synaptic transmission that is mostly involved in the elevation of second messengers (such as $\mathrm{Ca}^{2+}$, cAMP, or diacylglycerol). Second messengers directly or indirectly modulate the activity of kinases and phosphatases (Greengard, 2001). At the nerve terminal, elevation of $\mathrm{Ca}^{2+}$ concentration occurs upon arrival of an action potential and subsequent depolarization of the membrane. In order to discriminate between the effect of $\mathrm{Ca}^{2+}$ elevation and membrane depolarization on protein phosphorylation, we have designed three stimulation protocols of synaptosomes as described in the section 3.2.2. Our results clearly demonstrate that more phosphosites 
undergo phosphorylation/dephosphorylation upon depolarization of the membrane in the presence of $\mathrm{Ca}^{2+}$ ions (compare Figure 3-10 A, B with panel C). Moreover, phosphorylation changes of proteins are more pronounced compared to $\mathrm{Ca}^{2+}$-depleted samples. This led us to the conclusion that $\mathrm{Ca}^{2+}$ plays a greater role than membrane depolarization in the regulation of protein phosphorylation at the presynaptic terminal. This observation is in line with the known fact that $\mathrm{Ca}^{2+}$ directly or indirectly modulates the activity of kinases and phosphatases. For instance, it binds (solely or in complex with calmodulin) to the calcium sensor domains of CaMKII and conventional PKC isoforms as well as protein phosphatase 2B (Calcineurin) and increases their enzymatic activity. Moreover, it is known that $\mathrm{Ca}^{2+}$ activates $\mathrm{Ca}^{2+}$-dependent phospholipases and adenylate cyclases, which consequently elevates the concentration of other second messengers such as DAG (Kadamur and Ross, 2013) and cAMP (Halls and Cooper, 2011) and increases the activity of PKC isoforms (conventional and novel isoformes) and PKA, respectively. Each of the mentioned enzymes that are activated by $\mathrm{Ca}^{2+}$ in turn also modulate the activity of other kinases and phosphatases. For example, it is known that CaMKII increases the activity of ERK1/2, while it inhibits GSK-3 (see section 4.4 for more details).

\subsubsection{Impact of active zone proteins phosphorylation on synaptic transmission}

One of the major findings of this study is the extensive phosphorylation changes of active zone proteins upon stimulation (see section 3.2.3.1). A growing body of evidence have suggested that active zone proteins are involved in SV docking and the recruitment of calcium channels to the active zone (Han et al., 2011, Davydova et al., 2014, Nishimune et al., 2012). The number and the pattern of phosphosites changes are diverse among the component of active zone proteins. In case of bassoon, dephosphorylation was more pronounced upon stimulation as compared to other active zone proteins. This is particularly interesting considering that bassoon is known to speed up the rate of vesicle reloading at the active zones (Hallermann et al., 2010). Therefore, it can be speculated that vast dephosphorylation of bassoon upon stimulation modulates neurotransmission via regulation of SV docking. Although bassoon and piccolo share structural homology, they have unique segments that imply different functions. Piccolo binds to actin-related proteins and regulates F-actin assembly in presynapse and consequently synaptic transmission (Waites et al., 2011). 
Interestingly, in our dataset piccolo was not dephosphorylated as extensively as bassoon. This led us to conclude that phosphorylation status of bassoon plays a greater role in the regulation of vesicle docking as compared to piccolo.

Phosphorylation of active zone proteins may also regulate the SV pools size. Recently it was reported that ELKS proteins are involved in the increase of RRP pool size via its $\mathrm{N}$-terminus (Held et al., 2016) and interestingly in this study, we have quantified two phosphosites at the $\mathrm{N}$-terminus of ELKS proteins that are extensively dephosphorylated upon stimulation. RIM is the central organizer of the active zone and is also known to increase the RRP pool size and tether the $\mathrm{N}$ - and $\mathrm{P} / \mathrm{Q}$-type $\mathrm{Ca}^{2+}$-channels to the active zone via direct interaction through PDZ domain (Kaeser et al., 2011b). In our dataset, we quantified several sites distributed through all RIM domains. It is predictable that phosphorylation alters the protein-protein interaction of RIM and consequently modulates neurotransmission.

\subsubsection{Phosphorylation of cytoskeleton-associated proteins regulates exo-and endocytosis}

In our dataset phosphorylation of cytoskeleton-associated proteins has been observed to be largely modulated by stimulation and inhibition of $\mathrm{Ca}^{2+}$-dependent kinases and phosphatases (see Figures 3-12, 3-19 B, 3-23 B). Between the cytoskeleton filaments, the role of actin

filaments in neurotransmitters release has been more extensively investigated. The organization of actin filaments has been reported to have a significant role in both exo- and endocytosis.

Upon prolonged synaptic activity, the RRP pool has to be replenished by recycling SVs pool. It has been suggested that actin filaments act as a trap for SVs and their disassembly upon stimulation facilitate the RRP pool replenishment. In our dataset, we have quantified phosphosites of actin-related proteins that are involved in the reorganization of actin filaments such as Drebin and Adductin. The phosphorylation of these proteins has been reported to modulate their binding to actin filaments which in turn results in reorganization of the filaments (Worth et al., 2013). The actin-binding motor proteins (myosins) have been also reported to be involved in the movement of SVs at the nerve terminals (Seabrooke and Stewart, 2011). Our observation regarding the regulation of myosin phosphorylation upon stimulation shows that it might have a role in the modulation of SV movement. 
Actin filaments are reported to have a controversial effect on endocytosis. On one hand, it is suggested that they function as a trap for the components of endocytic machinery and prevent their spatial movements (Gaidarov et al., 1999), facilitate the invagination of the coated pit (Schmidt and Huttner, 1998) and enhance the fission of coated pits via polymerization around the neck of the SV pits (Lamaze et al., 1997). On the other hand, the rigid actin filaments may play a role as the barrier for the invagination of coated pits (Fujimoto et al., 2000). All the mentioned functions rely on the organization of actin filaments and, therefore, it is feasible that phosphorylation of actin-binding proteins indirectly regulates endocytosis.

\subsubsection{Multisite phosphorylated proteins}

As it was mentioned before, we have identified proteins, which contain multiple phosphorylation sites that simultaneously undergo opposing changes in their phosphorylation status such as bassoon, piccolo, and RIM. Two fundamental mechanisms for multiple phosphorylations have been reported (Roach, 1991): 1) The substrate has multiple recognition sites for one or more kinases. For instance, neurofilaments have multiple KSP motifs that are targeted by various proline-directed kinases (Holmgren et al., 2012). 2) Phosphorylation of one phosphosite influences the probability of phosphorylation on the other sites. The classical example is glycogen synthase which is not a substrate of GSK-3 when it is completely dephosphorylated. However, upon phosphorylation by CK2 at a specific site, four other sites become available for phosphorylation by GSK-3 (Fiol et al., 1990). The latter mechanism might explain our observation of highly coordinated phosphorylation and dephosphorylation of active zone proteins in the resting and stimulated conditions, especially considering that almost $20 \%$ and $50 \%$ of active zone phosphosites was contain the GSK-3 (SxxxSP) and proline-directed kinases (SP) consensus motifs, respectively (see Figure 3-20). Another example that is more related to this project is the phosphorylation of dynamin-1 at S774 and S778. It is known that S774 is phosphorylated by GSK-3 only if S778 was previously phosphorylated by CdK5 (Clayton et al., 2010).

Also, it has been reported that phosphorylation at one site might induce the conformational changes in the structure of the substrate that makes it recognizable for another kinase. In this mechanism, phosphate group does not participate in the kinase-substrate recognition. 
DARPP-32, the potential inhibitor of PP1, is a well-known example of this mechanism. Phosphorylation of DARPP-32 at T34 is enhanced when it is phosphorylated beforehand at S102 by CK2 (Greengard et al., 1999). We have observed this phenomenon in our dataset as well (see 3.3.2, Kinases and phosphatases section).

Beside the mechanism of multisite phosphorylation, another question that is commonly raised is why proteins are multiply phosphorylated? One explanation is that several phosphorylation events generate a variety of protein forms that have a variety of functions. Theoretically, a protein with ' $\mathrm{n}$ ' number of phosphosites can have $2^{\mathrm{n}}$ forms that can have several functions or grades of a single function (Salazar and Höfer, 2009). For instance, different phosphorylation states of bassoon might be involved in the gradual release of SVs upon strong stimulation over time. In case of enzymes, some of the phosphorylation sites alter the enzymatic activity, while others regulate the enzyme localization (Kim et al., 2015). Furthermore, it can be speculated that multiple phosphorylations are needed for major conformation changes of proteins which cannot happen by a single phosphorylation event. For example, it is known that phosphorylation of MARCKS by PKC increases its surface negative charge and abrogates its interaction with the membrane (Arbuzova et al., 2002).

\subsection{Possible role of Calcineurin in RRP pool replenishment and kinase regulation}

The role of Calcineurin in endocytosis has been studied extensively. It is known that Calcineurin dephosphorylates proteins involved in clathrin-mediated endocytosis (collectively called dephosphins) in an activity-dependent manner and also enhances endocytosis in the nerve terminal (Marks and McMahon, 1998, Cousin et al., 2001). Our results are consistent with both previous observations: major endocytotic proteins are dephosphorylated upon stimulation (see section 3.2.3.2) and Calcineurin inhibition, abolishes their dephosphorylation (see section 3.3.1). Interestingly, we have observed that active zone phosphosites were affected more than endocytosis related sites upon Calcineurin inhibition and, interestingly, many of them were the sites that were dephosphorylated upon stimulation (see Figure 3-13). Therefore, it seems that the cycle of phosphorylation/dephosphorylation of active zone phosphosites is very similar to the dephosphins and both are the substrates of Calcineurin during stimulation. Among active 
zone proteins, bassoon contains the highest number of downregulated sites upon stimulation. It is also the putative substrate of Calcineurin. It is known that many of Calcineurin substrates have the Calcineurin binding motifs (PxIxIT and LxVP) (Rodriguez et al., 2009). In line with our data, we found these motifs in bassoon, piccolo, RIM and Munc13 (active zone proteins containing phosphosites affected by Calcineurin inhibition), whereas the same motifs were absent in ERC1/2, which have no phosphosites affected by Calcineurin. Since bassoon is involved in the replenishment of RRP pool during and after stimulation (Hallermann et al., 2010, Mendoza Schulz et al., 2014), it is tempting to speculate that Calcineurin contributes to SV docking and the replenishment of RRP pool via dephosphorylation of bassoon upon stimulation. However, no evidence support a direct interaction between bassoon and SVs. Thus, this effect is likely mediated by an adapter protein. In fact, it has been reported that Mover protein links bassoon to SVs. It interacts with bassoon (Kremer et al., 2007) and also in a phosphorylation-dependent manner with SVs (Ahmed et al., 2013). Also, it has been proposed that Mover regulates the release probability via the $\mathrm{Ca}^{2+}$-dependent interaction with CaM (Korber et al., 2015). However, in our dataset, we quantified one phosphosite of Mover (T34) that was not regulated upon stimulation and no function has been reported for it.

Using the Calcineurin inhibitor and analysis of the up and downregulated phosphosites motifs, I have observed that Calcineurin inhibition has a dual effect on the phosphorylation of 'SP' motif, due to the enrichment of this motif in both up and downregulated sites. This can be because Calcineurin has diverse effects on the proline-directed kinases. Three main proline-directed kinases (ERK1/2, GSK-3 and CdK5) are known to be present in the nerve terminal. In fact, downregulation of two activating phosphosites of ERK1/2 (T183, Y185) upon Calcineurin inhibition clearly indicates that ERK1/2 and Calcineurin are co-deactivated. This observation has been also reported for pancreatic $\beta$ cells (Duan and Cobb, 2010). In contrast to ERK1/2, consensus motif (SxxxSP) of another proline-directed enzyme, GSK-3, was enriched in upregulated phosphosites. It is known that GSK-3 is constantly active unless

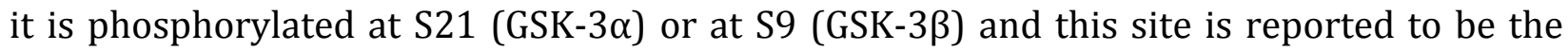
substrate of a variety of phosphatases (PP1, PP2A, and PP2B) (Hernandez et al., 2010, Kim et al., 2009). Here, we cannot relate the dephosphorylation of S21/S9 of GSK-3 to the activity of Calcineurin (otherwise the phosphosites with SxxxSP motif should be enriched in the 
downregulated sites). Another explanation for this observation is that since GSK-3 needs a priming kinase for phosphorylation of its substrate (see introduction section 1.3.3.7), downregulation of other kinases results in the inhibition of GSK-3 activity. Two enzymes have been reported to function as the primary kinase for GSK-3, the CK2 and CdK5 (Fiol et al., 1990, Clayton et al., 2010). Between these two enzymes, CK2 is less likely to be the primary kinase for GSK-3, since it is upregulated upon Calcineurin inhibition (see section 3.3.1). Therefore, it can be suggested that CdK5 is the primary kinase for GSK-3 and the downregulation of its activity results in the decrease of GSK-3 substrate phosphorylation.

\subsection{CaMKII might function as the major regulator of kinases/phosphatases upon stimulation}

The best-studied function of CaMKII in the presynapse is the activity-dependent phosphorylation of synapsin which results in the disassociation of SVs from the actin filaments. However, our observation suggests that CaMKII might have another important function in the nerve terminal as the regulator of other kinases and phosphatases upon stimulation. Application of pharmacological inhibitor of CaMKII resulted in immense downregulation of phosphosites in the nerve terminal. More interestingly, many of these sites do not have the consensus motif of CaMKII (R/KxxS). Many of these sites contained the 'SP' or 'SxE/DE motifs (see section 3.3.2). In the same line, it was observed that phosphosites belong to 'Kinases and Phosphatases' group are highly affected by CaMKII inhibition. These sites are mentioned in the section 3.3.2. Here I will discuss their phosphorylation regulation in more detail.

Upon CaMKII inhibition, we have observed the downregulation of autophosphorylation sites of CaMKII that decreases its activity (S279) or makes it independent from the $\mathrm{Ca}^{2+}$ concentration (T286). Moreover, we have detected the downregulation of activating threonine residues in the activation loop of PKA (T198) and PKC $\alpha$ (T497) upon CaMKII inhibition. Both of these sites are reported to be the substrates of phosphoinositidedependent kinase 1 (PDK-1). PDK-1 activity is upregulated upon phosphorylation at S241. Therefore, it is conceivable that CaMKII increases the activity of PKA and PKC via

phosphorylation of PDK-1 at S241. To test this hypothesis, I have checked the phosphorylation status of PDK-1 at S241 as well as PKA (T198) and PKC $\alpha$ (T497) by 
phospho-specific antibodies against these sites (Figure 4-1). The results confirmed our hypothesis, showing the downregulation of PDK-1 at S241, PKA at T198 and PKC $\alpha$ at T497 upon treatment with KN-93.

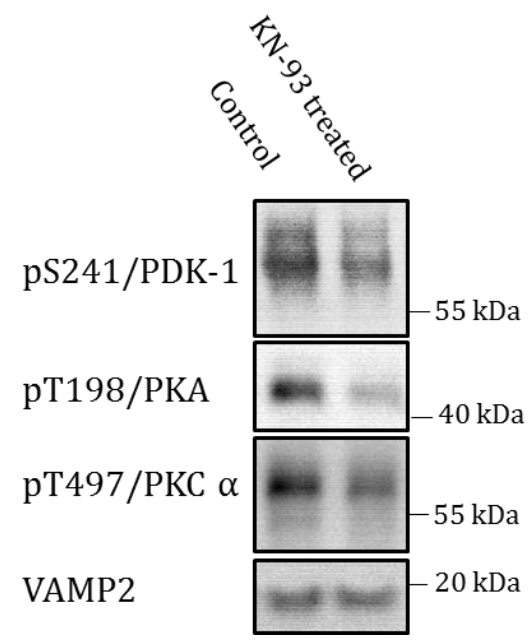

Figure 4-1: CaMKII inhibition downregulates PKA and PKC activity indirectly through the downregulation of PDK-1.

The inhibition of CaMKII by KN-93 decreases the phosphorylation of PDK-1 at S102, resulting in the downregulation of its activity. Consequently the phosphorylation of PKA at T198 and PKCa at T497 (both known to be substrates of PDK-1) is decreased, which in return decreases the activity of PKA and PKC. For the loading control antibody against VAMP2 was used.

In our dataset, we were not able to find any direct evidence regarding the downregulation of proline-directed kinases upon CaMKII inhibition. However, it has been reported that ERK1/2 is activated via PKA (Sindreu et al., 2007) and CaMKII (Salzano et al., 2012) via phosphorylation of Raf1. That is why, it can be suggested that inhibition of CaMKII directly or indirectly via inhibition of PKA downregulates Raf-1 activity and consequently downregulates ERK1/2.

Another kinase that was observed to be downregulated upon CaMKII inhibition is CK2, due to the enrichment of 'SxEE' and 'SxD' motifs among the downregulated phosphosites. However, the mechanism of CK2 regulation by CaMKII is not known. Moreover, as was described in the previous section, CK2 may function as the priming kinase for GSK-3 and its inhibition may downregulate the phosphorylation of GSK-3 substrates.

Also, the CK2 downregulation indirectly increases the activity of PP1 via DARPP-32. In our dataset, we have observed the decrease in DARPP-32 (S102) phosphorylation. This site is known to be the substrate of CK2 and in phosphorylated status it increases the availability of DARPP-32 to be phosphorylated by PKA at T34 (Greengard, 2001). Phosphorylation at T34 
makes DARPP-32 a potent inhibitor of PP1. Altogether, it can be concluded that downregulation of CaMKII-CK2-DARPP-32 (S102 and T34) pathway increases the activity of PP1.

The CaMKII inhibition also diminished the activity of MARK kinase via downregulation of phosphorylation at T215. MARK is known to target microtubule-associated proteins and its downregulation can partially explain the extensive downregulation of cytoskeletonassociated phosphosites upon CaMKII inhibition (see Figure 3-23 B).

Overall, inhibition of several kinases (CaMKII, PKA, PKC, GSK-3, ERK1/2, CK2 and MARK) and upregulation of PP1, explains the mechanism of extensive downregulation of phosphosites upon CaMKII inhibition. Also, the fact that upon membrane depolarization the concertation of $\mathrm{Ca}^{2+}$ increases and among the kinases, only CaMKII and conventional isoforms of PKC are able to directly sense the $\mathrm{Ca}^{2+}$ elevation supports the idea that CaMKII might function as the major regulator of other kinases and phosphatases in the nerve terminal upon stimulation.

\subsection{Activity of kinases and phosphatases is tightly coordinated in the nerve terminal}

As it was discussed in the previous sections, the activity of the kinases and phosphatases is highly interconnected with each other and their inhibition (e.g. Calcineurin and CaMKII) affects the activity of other kinases and phosphatases. An important question is how these enzymes (kinases/phosphatases), second messengers ( $\left.\mathrm{Ca}^{2+}, \mathrm{cAMP}, \mathrm{DAG}\right)$ and the substrates are organized in the nerve terminal and are able to coordinate in a fast, localized and specific manner upon stimulation.

As it was described before, it is highly likely that $\mathrm{Ca}^{2+}$ ions are the main second messengers that is upregulated upon stimulation. This elevation occurs via the voltage-gated calcium channels that are enriched in the active zone through proteins such as RIM, RIM-binding protein, and ELKS. Therefore, it would be conceivable $\mathrm{Ca}^{2+}$ sensor proteins are also localized in the vicinity of the active zones. One of the mechanisms that has been described to localize the $\mathrm{Ca}^{2+}$-dependent kinases/phosphatases close to the plasma membrane is the network of scaffolding proteins. This mechanism, has been investigated in the postsynapse where the Akinase anchoring protein (AKAP79/150) binds to PKA, PKC, Calcineurin (Sanderson and Dell'Acqua, 2011) and enzymes that produce the second messengers such as adenylate 
cyclase (Zhang et al., 2013a) to keep them close to postsynaptic density. In our dataset, we could detect phosphosites of AKAP12 and AKAP5 in the nerve terminal. Overall, it can be predicted that a similar mechanism (through AKAPs or other scaffolding proteins) localizes the components of $\mathrm{Ca}^{2+}$ signaling molecules close to the active zone in presynapse.

The other possible mechanism for compartmentalization of kinases/phosphatases is binding to actin filaments. It is known that actin filaments are localized close to the active zone (Doussau and Augustine, 2000). For instance, PP1 binds to actin filaments via Neurabin protein. This interaction is highly dependent on the phosphorylation of Neurabin at S100 and decreases the binding of PP1 to actin filaments leading to its diffusion away from the nerve terminal (McAvoy et al., 1999).

Another mechanism for the coordination of the kinases and phosphatases activity is the overlap between their regulators. The best-known example of this mechanism in neurons is DARPP-32. Depending on phosphorylation status, DARPP-32 can be a potent inhibitor of either PP1 or PKA. As it was discussed before DARPP-32 is phosphorylated by PKA at T34 to inhibit PP1 inhibitor, whereas when it is phosphorylated at T75 by CdK5 it becomes the inhibitor of PKA. Many other kinases and phosphatases (CK1, CK2, PP2A, and Calcineurin) modulate the phosphorylation status of DARPP-32 towards ones that mentioned and coordinate its inhibitory function with the external signal arriving at the neuron (Greengard, 2001). The other example of this mechanism, is the phosphorylation of both PKA and PKC at threonine in their activation loop by PDK-1, resulting in the co-regulation of PKA and PKC activity.

Overall, based on our observations regarding the autoregulation of kinases/phosphatases activity (see section 3.3) as well as the regulation of protein phosphorylation upon stimulation (see section 3.2), I propose a model in which $\mathrm{Ca}^{2+}$ is the primary second messenger upon stimulation that modulates the activity of tightly interconnected kinases/phosphatases (Figure 4-2). 


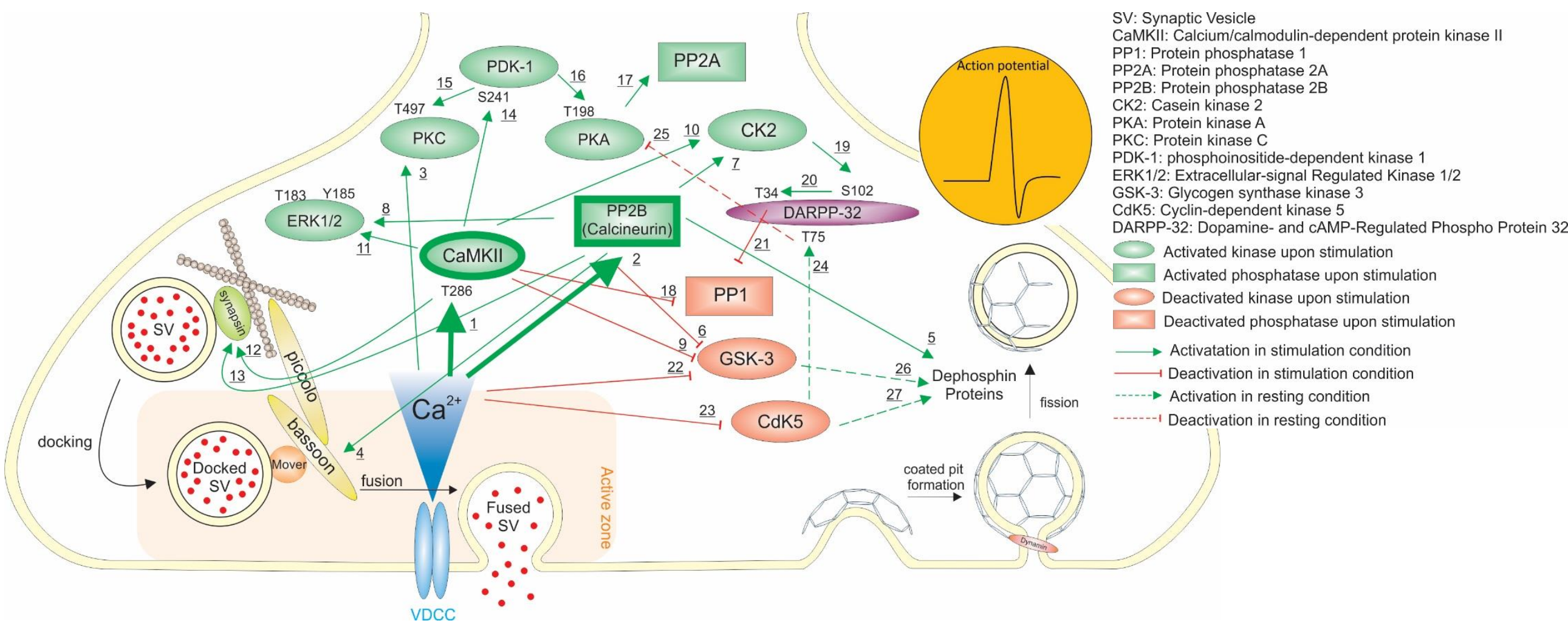

Figure 4-2: Activity of kinases and phosphatases is tightly coordinated in the nerve terminal upon stimulation.

Upon arrival of action potential to the nerve terminal, CaMKII, Calcineurin and conventional PKCs are activated by $\mathrm{Ca}^{2+}$ elevation $(1,2$, and 3$)$. Calcineurin dephosphorylates active zone and clathrin-mediated endocytosis (dephosphins) phosphosites (Figures 3-13, 3-14 A, 3-19 B) (ㄴ,5), inhibits the activity of GSK-3 (Figures 3-20 A and B) (므), increases the activity of CK2 (Figures 3-21) (ㅁ) and ERK1/2 (phosphorylation of T183 and Y185) (ㅁ). CaMKII inhibits the activity of GSK-3 (Song et al., 2010) (9)), increases the activity of CK2 (Figures 3-24, A and B) (10) and ERK1/2 (phosphorylation of T183 and Y185) (11). The activated CaMKII and Calcineurin phosphorylate synapsin (at S566 andS603) and dephosphorylate (at S436, S508, S549, and S551), resulting in disassociation of SVs from actin filaments (Figure 3-15) (12,13). CaMKII also increases the activity of PKA (phosphorylation of T198) and PKC (phosphorylation of T497), probably via activation of PDK-1 (see section 4-3) (14,15, and 16). PKA increases the activity of PP2A via phosphorylation of B56 $\delta$ regulatory subunit at S567 (see section 3.2.4) (17). Moreover, CaMKII decreases the activity of PP1 in the nerve terminal by phosphorylation of its regulatory subunit (Neurabin-2) at S100 (see section 3.2.4) (18). The activated CK2 increases the phosphorylation of DARPP-32 at S102 and consequently phosphorylation of DARPP-32 at T34, converting it into the inhibitor of PP1 (see section 3.3.2) (19,20, and 21). Upon $\mathrm{Ca}^{2+}$ elevation, the substrates of proline-directed kinases (GSK-3 and CdK5) are dephosphorylated probably via activation of CaMKII and Calcineurin (see number $\underline{6}$ and $\underline{9})(\underline{22}, 23)$. In the resting condition, CdK5 is more active and phosphorylates DARPP-32 at T75, converting it into the inhibitor of PKA (Greengard, 2001) $(24,25)$. In the resting condition, CdK5 and GSK-3 rephosphorylate the clathrin-mediated endocytosis (dephosphins) (26,27). Adapted and modified from (Chua et al., 2010). 


\subsection{Experimental approach}

\subsubsection{Stimulation of synaptosomes \\ KCl stimulation}

In this study, a high concentration of $\mathrm{KCl}(50 \mathrm{mM})$ was used to stimulate synaptosomes and to release neurotransmitters. $\mathrm{KCl}$ stimulation has advantages and disadvantages that I will discuss in this section. The main limitation of $\mathrm{KCl}$ stimulation is that it leads to a stable depolarization of the membrane and is not able to mimic the repetitive transient depolarization similar to what nerve terminals are subjected to in vivo (Tibbs et al., 1989). The membrane depolarization by $\mathrm{KCl}$ results in influx of $\mathrm{Ca}^{2+}$ to the nerve-terminal through voltage-dependent calcium channels and also through the reversal of $\mathrm{Na}^{+} / \mathrm{Ca}^{2+}$ exchanger (Carvalho et al., 1988). It is known that $\mathrm{KCl}$ stimulation produces a spike in $\mathrm{Ca}^{2+}$ concentration 1-5 seconds after the stimulation, which recovers to a plateau that lasts for minutes (McMahon and Nicholls, 1991). Therefore, the influx of $\mathrm{Ca}^{2+}$ ions in the nerve terminal is higher as compared to a physiological condition and remains at the high level for a much longer time.

One the other hand, $\mathrm{KCl}$ stimulation is reported to be very robust to initiate the glutamate release and decreases the membrane potential noise (Tibbs et al., 1989). Also, most of the principal knowledge regarding the activity-dependent phosphorylation of synaptosomal proteins have been based on $\mathrm{KCl}$ stimulation that enabled us to prove the functionality of our approach by comparing our result to them. More importantly, substantial elevation of $\mathrm{Ca}^{2+}$ concentration in the nerve terminal by $\mathrm{KCl}$ stimulation made the $\mathrm{Ca}^{2+}$-dependent phosphorylation/dephosphorylation events more pronounced, resulting in the increase of signal to noise ratio and in turn easier detection of phosphorylation changes.

\section{Electrical stimulation}

Electrical stimulation has been used previously to investigate the long-term metabolic changes of synaptosome upon stimulation (Rodina et al., 1980) and, in contrast to $\mathrm{KCl}$ stimulation, very little knowledge are available regarding the neurotransmitter release upon electrical stimulation. We have set up this method to overcome the drawbacks of $\mathrm{KCl}$ stimulation. Using electrical stimulation, it is possible to control the membrane depolarization through the frequency and the number of action potentials (APs). As it is 
shown in Figure 4-3, the release correlates with the number of APs. Also, it is responsive to the absence of external $\mathrm{Ca}^{2+}$ ions (using EGTA) and application of $\mathrm{Na}^{+}$channel blocker (Tetrodotoxin) (data are not shown). Altogether, this shows that the structure of synaptosomes remains intact during the electrical stimulation. The main advantage of the electrical stimulation is that it is possible to target distinct SV pools. It is known that RRP and recycling pools can be released by $40 \mathrm{AP}$ at $20 \mathrm{~Hz}$ (Schikorski and Stevens, 2001, Murthy and Stevens, 1999) and $600 \mathrm{AP}$ at $20 \mathrm{~Hz}$ (Opazo et al., 2010, Fernandez-Alfonso and Ryan, 2008), respectively. I have tried these stimulation protocols on synaptosomes and observed that the release of glutamate by $\mathrm{KCl}$ stimulation after 2 min (the time point that we have collected synaptosomes for the protein phosphorylation analysis) is similar to the release of RRP by $40 \mathrm{AP}$ at $20 \mathrm{~Hz}$. However, the kinetics of the release with $\mathrm{KCl}$ stimulation is much slower than electrical stimulation (see Figure 4-3). One reasonable explanation is the inevitable mixing artifact caused by the large sample volume ( $2 \mathrm{ml}$ cuvette). In fact, to avoid this problem a rapid mixing device with perfusion system has been developed in order to measure the biochemical parameters of release in the initial phase (Leenders et al., 1999) in synaptosomes.

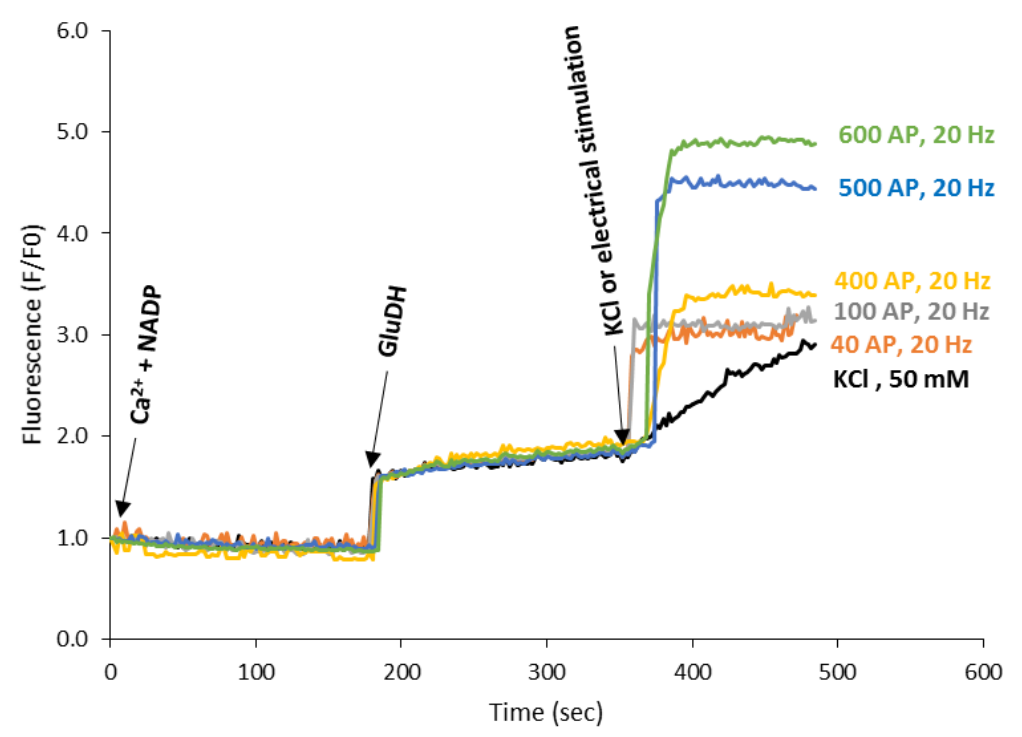

Figure 4-3: $\mathrm{KCl}$ stimulation versus electrical stimulation.

The release of glutamate from synaptosomes was investigated upon $\mathrm{KCl}$ stimulation and electrical stimulation $(7 \mathrm{~V})$ with different number of action potentials (AP) in the first $2 \mathrm{~min} . \mathrm{KCl}(50 \mathrm{mM})$ stimulation could release the glutamate at almost the same levels as $40 \mathrm{AP}, 20 \mathrm{~Hz}$ which is known to be enough for the release of readily releasable pool (RRP) of synaptic vesicles. The release was much lower comparing to $600 \mathrm{AP}, 20 \mathrm{~Hz}$ stimulation that is typically required for the release of recycling pool. The kinetics of the release by $\mathrm{KCl}$ stimulation was much slower than electrical stimulation. Two minute stimulation by $\mathrm{KCl}$ releases glutamate as much as 2 seconds electrical stimulation ( $40 \mathrm{AP}, 20 \mathrm{~Hz}$ ). 


\subsubsection{Time-course of stimulation-dependent phosphorylation changes}

As it was mentioned before, in this study we have collected samples for the analysis of protein phosphorylation changes, 2 min after stimulation by $\mathrm{KCl}$. This raised several questions. How does the kinetics of the phosphorylation changes relate to neurotransmitter release? Is there any order in the activation of kinases or phosphatases? Is there any pattern in the regulation of effector proteins in SV recycling?

Answers to all the questions mentioned above would enhance our knowledge of the regulatory machine of neurotransmission. Unfortunately, the experimental approach that was used in this study is not efficient to answer them. The primary reason for that is the clamped depolarization by $\mathrm{KCl}$. $\mathrm{KCl}$ stimulation elevates $\mathrm{Ca}^{2+}$ concentration in the nerve terminal for a long period of time (McMahon and Nicholls, 1991). In the physiological condition, $\mathrm{Ca}^{2+}$ concentration is buffered very fast (Scott, 2007). This is important when we keep in mind that $\mathrm{Ca}^{2+}$ ions are the key second messenger that is elevated by the arrival of action potential and directly modulates the activity of $\mathrm{Ca}^{2+}$-dependent kinases/phosphatases. Moreover, based on our observations and previous reports $\mathrm{Ca}^{2+}$-dependent kinases/phosphatases are involved in the regulation of other kinases and phosphatases (see section 4.2 and 4.3). For instance, CaMKII is known to be activated upon the elevation of $\mathrm{Ca}^{2+}$ and, only if it is exposed for a longer time to the elevated $\mathrm{Ca}^{2+}$ ion condition phosphorylates itself that makes it insensitive to $\mathrm{Ca}^{2+}$ concentration (Lisman et al., 2002). Therefore, it is conceivable that CaMKII is highly activated over the time course of clamped depolarization induced by $\mathrm{KCl}$. To test this, the phosphorylation status of synapsin pS603 (known as a substrate of CaMKII) was assessed by immunoblotting when synaptosomes were stimulated by $\mathrm{KCl}$ and electrical stimulation (Figure 4-4 B). Two conclusions can be drawn from this result, first, the kinetics of synapsin1 phosphorylation is different in $\mathrm{KCl}$ stimulation compared to the electrical stimulation. In $\mathrm{KCl}$ stimulation, the upregulation of phosphorylation increased $2 \mathrm{sec}$ after and remained at a high level even after $10 \mathrm{~min}$, whereas in case of electrical stimulation the phosphorylation increased after $10 \mathrm{sec}$ and clearly decreased $30 \mathrm{sec}$ after stimulation. Second, the extent of phosphorylation is high in the $\mathrm{KCl}$ stimulation compared to the electrical stimulation. This can be explained by the known fact that $\mathrm{KCl}$ stimulation overloads synaptosomes with $\mathrm{Ca}^{2+}$ ions through both voltage-dependent calcium channels and reverse $\mathrm{Na}^{+} / \mathrm{Ca}^{2+}$ exchanger (Carvalho et al., 1988). 
Similarly, dynamin1 pS774 (the known substrate of Calcineurin) is dephosphorylated faster and more extensively by $\mathrm{KCl}$ stimulation rather than electrical stimulation. Another reason that makes the $\mathrm{KCl}$ stimulation not suitable for the time-course study of phosphorylation changes is the mixing effect (see previous section). The glutamate release by $\mathrm{KCl}$ stimulation is clearly slower than during the electrical stimulation. The amount of glutamate that is released by $\mathrm{KCl}$ stimulation in $10 \mathrm{~min}$ is almost the same as released by electrical stimulation in $30 \mathrm{sec}$ with $600 \mathrm{AP}(20 \mathrm{~Hz})$ (required to release the recycling pool) (Figure 4-4 A). Overall, $\mathrm{KCl}$ stimulation is not suitable for the time-course investigation of phosphorylation changes and does not resemble the fast and repetitive stimulation that neurons encounter under physiological condition.

\subsubsection{Mass-spectrometry approach}

Mass spectrometry-based phosphoproteomics is a powerful approach for analysis of largescale protein phosphorylation without having any prior knowledge about them. In this study, we took advantage of this method to map the phosphorylation changes of presynaptic proteins. Despite the fact that we were not able to detect all presynaptic phosphosites in our experiments, our dataset provides a comprehensive outlook at phosphorylation events taking place in the presynapse upon stimulation. Inability to detect higher amount of phosphosites is dictated by several technical limitations. First, we were limited to use $1 \mathrm{mg}$ of synaptosomal proteins as a starting material in stimulation reaction cuvette, whereas similar studies that were able to identify more phosphosites typically used much more starting material (15-30 mg) (Trinidad et al., 2012). Second, nerve terminals are enriched in the membrane proteins and solubilization of them in buffers suitable for mass spectrometry remained challenging. Several methods has been suggested to improve the recovery of digested peptides of membrane proteins. For instance the filter-aided sample preparation (FASP) protocol (Wisniewski et al., 2009), which takes the advantage of SDS to solubilize proteins and then high concentration of urea is used to wash out the SDS. However this method in our hand did not yield in more identification of phosphopeptides. Nevertheless, more optimization in digestion protocol of large amounts of membrane protein enriched samples would increase the recovery of the peptides and consequently identification of phosphopeptides. Third, insufficiency of one phosphopeptide enrichment method to capture 


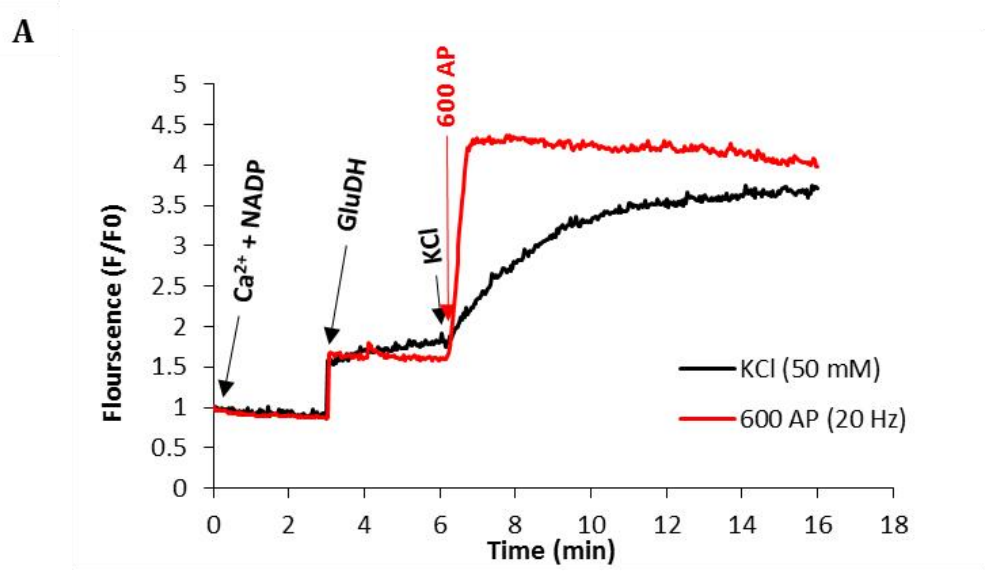

B
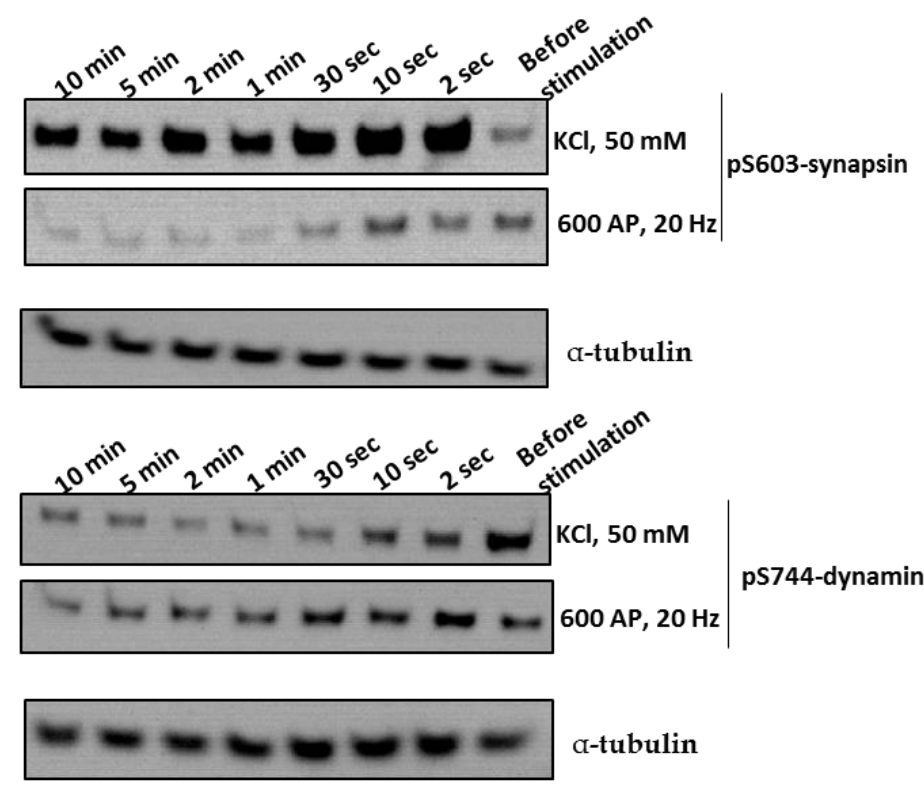

Figure 4-4: Dynamics of glutamate release and protein phosphorylation by $\mathrm{KCl}$ versus electrical stimulation.

(A) The release of glutamate from synaptosomes was investigated upon $\mathrm{KCl}$ stimulation and electrical stimulation (600 AP, Hz) for $10 \mathrm{~min}$. Electrical stimulation resulted in the fast and strong release of glutamate up to 4.5 folds of baseline in $30 \mathrm{sec}$. The release was stopped after $30 \mathrm{sec}$ and slowly decreased over time, possibly due to the reverse reaction of glutamate dehydrogenase. $\mathrm{KCl}$ stimulation resulted in the slow release of glutamate over $10 \mathrm{~min}$, possibly due to the mixing effect. At the beginning the release was faster and after almost 3 minutes it reached a plateau and was not decreasing later, due to $\mathrm{KCl}$ clamp depolarization of the membrane. (B) Immunoblot analysis of synaptosome upon $\mathrm{KCl}$ and electrical stimulation for synapsin pS603 and dynamin pS774 over 10 mins. Both phosphosites were affected by $\mathrm{KCl}$ stimulation faster and to a higher extent. Synapsin pS603 was upregulated strongly by $\mathrm{KCl}$ stimulation after 2 sec and remained highly phosphorylated over $10 \mathrm{~min}$. With electrical stimulation, slight upregulation happened $10 \mathrm{sec}$ after stimulation and decreased to the basal level after $30 \mathrm{sec}$. Dynamin pS774 was downregulated strongly with $\mathrm{KCl}$ stimulation after $2 \mathrm{sec}$. With electrical stimulation phosphorylation of dynamin S774 was decreased slightly and after 30 sec. Antibody against a-tubulin was used for the loading control. 
all the phosphopeptides. It has been shown that different enrichment methods are partially biased towards a distinct population of phosphosites. For example, it has been reported that IMAC enrichment is more efficient for the enrichment of multiply phosphorylated peptides comparing to $\mathrm{TiO}_{2}$ method (Yue et al., 2015). Ideally, a combination of several enrichment methods can provide a more comprehensive map of phosphosites. Moreover, as it was discussed in the Results part, use of dimethyl-labeling led to the decreased number of quantified phosphosites (see section 3.1.4). This is due to the known fact that the labeling increases complexity of samples at the MS1 level. Therefore, the decision to use labeling or label-free approach is a compromise between number of identified phosphosites and reliability of phosphopeptide quantification. However, recent development of quantification algorithms and software has increased the reliability of label-free approaches (Cox et al., 2014) and made it more popular in the field.

Another important point that needs to be discussed is the method that has been used to determine significantly regulated phosphosites. Two common practices are used in the field for this purpose. In the first approach, an arbitrary cut-off for the fold changes is applied (for instance, $\mathrm{H} / \mathrm{L}$ ratio $>2$ or $<0.5$ ) and phosphosites that have a fold change higher than the cutoff are considered as the regulated sites. It is common to use this method when researches have some information regarding the extent of changes for number of phosphosites or have a similar phosphoproteomics dataset to compare and make an educated guess regarding the cut-off. The second practice is to use a statistical approach for determination of phosphosites with the outlier H/L ratio. The latter method was used mostly in this study to avoid bias towards the dataset.

\subsection{Conclusions and perspectives}

Phosphoproteomics analysis of presynaptic proteins has a tremendous potential to disentangle the regulation of synaptic transmission. In this thesis, utilizing the combination of MS-based phosphoproteomics method and different nerve terminal stimulation conditions, we have shown the role of calcium influx to the nerve terminal in modulation of the phosphorylation changes. Moreover, we have uncovered the extensive activitydependent phosphorylation changes of active zone proteins. Using the pharmacological inhibitor of Calcineurin, I was able to determine its role in the dephosphorylation of active 
zone proteins upon stimulation. Also, in this study, I have proposed a model in which kinases and phosphatases coordinate tightly to facilitate the SV release and recycling upon stimulation. The dataset generated in this study can be used as the basis for functional analysis of single phosphosites or phosphoproteins.

The similar workflow in a combination with other kinases/phosphatases inhibitors can be used to investigate the role of these enzymes in synaptic transmission. In addition, using the electrical stimulation protocol established in this study, it is possible to determine the phosphorylation events that have a role in the regulation of readily releasable and recycling pools of SVs. It is predictable that in future through the improvement of the phosphopeptides enrichment methods, development of faster and more sensitive mass spectrometer instruments, as well as more advanced and sophisticated modeling tools, more phosphosites will be identified and synaptic transmission regulation via phosphorylation will be unrevealed in more detail. 


\section{References}

ADAMS, J. A., MCGLONE, M. L., GIBSON, R. \& TAYLOR, S. S. 1995. Phosphorylation modulates catalytic function and regulation in the cAMP-dependent protein kinase. Biochemistry, 34, 2447-54.

AGOSTINIS, P., GORIS, J., PINNA, L. A. \& MERLEVEDE, W. 1987. Regulation of casein kinase 2 by phosphorylation/dephosphorylation. Biochem J, 248, 785-9.

AHMED, S., WITTENMAYER, N., KREMER, T., HOEBER, J., KIRAN AKULA, A., URLAUB, H., ISLINGER, M., KIRSCH, J., DEAN, C. \& DRESBACH, T. 2013. Mover is a homomeric phospho-protein present on synaptic vesicles. PLoS One, 8, e63474.

AHN, J.-H., MCAVOY, T., RAKHILIN, S. V., NISHI, A., GREENGARD, P. \& NAIRN, A. C. 2007. Protein kinase A activates protein phosphatase $2 \mathrm{~A}$ by phosphorylation of the B56 $\delta$ subunit. Proceedings of the National Academy of Sciences, 104, 2979-2984.

ALABI, A. A. \& TSIEN, R. W. 2012. Synaptic vesicle pools and dynamics. Cold Spring Harb Perspect Biol, 4, a013680.

AMIN, N. D., ZHENG, Y. L., KESAVAPANY, S., KANUNGO, J., GUSZCZYNSKI, T., SIHAG, R. K., RUDRABHATLA, P., ALBERS, W., GRANT, P. \& PANT, H. C. 2008. Cyclin-dependent kinase 5 phosphorylation of human septin SEPT5 (hCDCrel-1) modulates exocytosis. J Neurosci, 28, 3631-43.

ARBUZOVA, A., SCHMITZ, A. A. \& VERGERES, G. 2002. Cross-talk unfolded: MARCKS proteins. Biochem J, 362, 1-12.

BALLIF, B. A., VILLEN, J., BEAUSOLEIL, S. A., SCHWARTZ, D. \& GYGI, S. P. 2004. Phosphoproteomic analysis of the developing mouse brain. Mol Cell Proteomics, 3, 1093-101.

BARCLAY, J. W., ALDEA, M., CRAIG, T. J., MORGAN, A. \& BURGOYNE, R. D. 2004. Regulation of the fusion pore conductance during exocytosis by cyclin-dependent kinase 5. J Biol Chem, 279, 41495-503.

BARCLAY, J. W., CRAIG, T. J., FISHER, R. J., CIUFO, L. F., EVANS, G. J., MORGAN, A. \& BURGOYNE, R. D. 2003. Phosphorylation of Munc18 by protein kinase $C$ regulates the kinetics of exocytosis. J Biol Chem, 278, 10538-45.

BEACH, J. R., LICATE, L. S., CRISH, J. F. \& EGELHOFF, T. T. 2011. Analysis of the role of Ser1/Ser2/Thr9 phosphorylation on myosin II assembly and function in live cells. BMC Cell Biol, 12, 52.

BELLUZZI, E., GONNELLI, A., CIRNARU, M. D., MARTE, A., PLOTEGHER, N., RUSSO, I., CIVIERO, L., COGO, S., CARRION, M. P., FRANCHIN, C., ARRIGONI, G., BELTRAMINI, M., BUBACCO, L., ONOFRI, F., PICCOLI, G. \& GREGGIO, E. 2016. LRRK2 phosphorylates pre-synaptic N-ethylmaleimide sensitive fusion (NSF) protein enhancing its ATPase activity and SNARE complex disassembling rate. Mol Neurodegener, 11, 1.

BENFENATI, F., NEYROZ, P., BAHLER, M., MASOTTI, L. \& GREENGARD, P. 1990. Time-resolved fluorescence study of the neuron-specific phosphoprotein synapsin I. Evidence for phosphorylation-dependent conformational changes. J Biol Chem, 265, 12584-95.

BETAPUDI, V. 2014. Life without double-headed non-muscle myosin II motor proteins. Front Chem, 2, 45.

BETAPUDI, V., RAI, V., BEACH, J. R. \& EGELHOFF, T. 2010. Novel regulation and dynamics of myosin II activation during epidermal wound responses. Exp Cell Res, 316, 980-91. 
BETZ, A., THAKUR, P., JUNGE, H. J., ASHERY, U., RHEE, J.-S., SCHEUSS, V., ROSENMUND, C., RETTIG, J. \& BROSE, N. 2001. Functional Interaction of the Active Zone Proteins Munc13-1 and RIM1 in Synaptic Vesicle Priming. Neuron, 30, 183-196.

BOERSEMA, P. J., AYE, T. T., VAN VEEN, T. A., HECK, A. J. \& MOHAMMED, S. 2008. Triplex protein quantification based on stable isotope labeling by peptide dimethylation applied to cell and tissue lysates. Proteomics, 8, 4624-32.

BOERSEMA, P. J., MOHAMMED, S. \& HECK, A. J. 2009a. Phosphopeptide fragmentation and analysis by mass spectrometry. J Mass Spectrom, 44, 861-78.

BOERSEMA, P. J., RAIJMAKERS, R., LEMEER, S., MOHAMMED, S. \& HECK, A. J. 2009b. Multiplex peptide stable isotope dimethyl labeling for quantitative proteomics. Nat Protoc, 4, 484-94.

BOLLMANN, J. H., SAKMANN, B. \& BORST, J. G. 2000. Calcium sensitivity of glutamate release in a calyx-type terminal. Science, 289, 953-7.

BONDARENKO, P. V., CHELIUS, D. \& SHALER, T. A. 2002. Identification and relative quantitation of protein mixtures by enzymatic digestion followed by capillary reversed-phase liquid chromatography-tandem mass spectrometry. Anal Chem, 74, 4741-9.

BOYKEN, J., GRøNBORG, M., RIEDEL, D., URLAUB, H., JAHN, R. \& CHUA, JOHN JIA E. 2013. Molecular Profiling of Synaptic Vesicle Docking Sites Reveals Novel Proteins but Few Differences between Glutamatergic and GABAergic Synapses. Neuron, 78, 285-297.

BRADFORD, H. F. 1970. Responses of synaptosomes to electrical stimulation. Biochemical Journal, 117, 36P.

BREUKEL, A. I. M., BESSELSEN, E. \& GHIJSEN, W. E. J. M. 1997. Synaptosomes. In: RAYNE, R. C. (ed.) Neurotransmitter Methods. Totowa, NJ: Springer New York.

BURLEY, J. R. \& SIHRA, T. S. 2000. A modulatory role for protein phosphatase 2B (calcineurin) in the regulation of Ca2+ entry. European Journal of Neuroscience, 12, 2881-2891.

CAENEPEEL, S., CHARYDCZAK, G., SUDARSANAM, S., HUNTER, T. \& MANNING, G. 2004. The mouse kinome: Discovery and comparative genomics of all mouse protein kinases. Proceedings of the National Academy of Sciences of the United States of America, 101, 11707-11712.

CARMICHAEL, D. F., GEAHLEN, R. L., ALLEN, S. M. \& KREBS, E. G. 1982. Type II regulatory subunit of cAMP-dependent protein kinase. Phosphorylation by casein kinase II at a site that is also phosphorylated in vivo. J Biol Chem, 257, 10440-5.

CARVALHO, A. P., SANTOS, M. S., HENRIQUES, A. O., TAVARES, P. \& CARVALHO, C. M. 1988. Calcium Channels and $\mathrm{Na}+\mathrm{Ca} 2+$ Exchange in Synaptosomes. In: ZIMMERMANN, $\mathrm{H}$. (ed.) Cellular and Molecular Basis of Synaptic Transmission. Berlin, Heidelberg: Springer Berlin Heidelberg.

CESCA, F., BALDELLI, P., VALTORTA, F. \& BENFENATI, F. 2010. The synapsins: key actors of synapse function and plasticity. Prog Neurobiol, 91, 313-48.

CHAN, P.-C., HSU, R. Y. C., LIU, C.-W., LAI, C.-C. \& CHEN, H.-C. 2014. Adducin-1 is essential for mitotic spindle assembly through its interaction with myosin-X. The Journal of Cell Biology, 204, 19-28.

CHAPMAN, E. R. 2008. How does synaptotagmin trigger neurotransmitter release? Annu Rev Biochem, 77, 615-41.

CHEN, H., SLEPNEV, V. I., DI FIORE, P. P. \& DE CAMILLI, P. 1999. The interaction of epsin and Eps15 with the clathrin adaptor AP-2 is inhibited by mitotic phosphorylation and 
enhanced by stimulation-dependent dephosphorylation in nerve terminals. $J$ Biol Chem, 274, 3257-60.

CHEN, Z., COOPER, B., KALLA, S., VAROQUEAUX, F. \& YOUNG, S. M., JR. 2013. The Munc13 proteins differentially regulate readily releasable pool dynamics and calciumdependent recovery at a central synapse. J Neurosci, 33, 8336-51.

CHENG, H.-C., QI, R. Z., PAUDEL, H. \& ZHU, H.-J. 2011. Regulation and Function of Protein Kinases and Phosphatases. Enzyme Research, 2011, 3.

CHENG, Y., ZHANG, Y. \& MCCAMMON, J. A. 2006. How does activation loop phosphorylation modulate catalytic activity in the cAMP-dependent protein kinase: a theoretical study. Protein Sci, 15, 672-83.

CHIN, D. \& MEANS, A. R. 2000. Calmodulin: a prototypical calcium sensor. Trends Cell Biol, 10, 322-8.

CHO, R. W., BUHL, L. K., VOLFSON, D., TRAN, A., LI, F., AKBERGENOVA, Y. \& LITTLETON, J. T. 2015. Phosphorylation of Complexin by PKA Regulates Activity-Dependent Spontaneous Neurotransmitter Release and Structural Synaptic Plasticity. Neuron, 88, 749-61.

CHOU, W. H., WANG, D., MCMAHON, T., QI, Z. H., SONG, M., ZHANG, C., SHOKAT, K. M. \& MESSING, R. O. 2010. GABAA receptor trafficking is regulated by protein kinase $\mathrm{C}$ (epsilon) and the N-ethylmaleimide-sensitive factor. J Neurosci, 30, 13955-65.

CHUA, J. J. E., KINDLER, S., BOYKEN, J. \& JAHN, R. 2010. The architecture of an excitatory synapse. Journal of Cell Science, 123, 819-823.

CIESLA, J., FRACZYK, T. \& RODE, W. 2011. Phosphorylation of basic amino acid residues in proteins: important but easily missed. Acta Biochim Pol, 58, 137-48.

CIJSOUW, T., WEBER, J. P., BROEKE, J. H., BROEK, J. A. C., SCHUT, D., KROON, T., SAARLOOS, I., VERHAGE, M. \& TOONEN, R. F. 2014. Munc18-1 redistributes in nerve terminals in an activity- and PKC-dependent manner. The Journal of Cell Biology, 204, 759-775.

CLAYTON, E. L., SUE, N., SMILLIE, K. J., O'LEARY, T., BACHE, N., CHEUNG, G., COLE, A. R., WYLLIE, D. J., SUTHERLAND, C., ROBINSON, P. J. \& COUSIN, M. A. 2010. Dynamin I phosphorylation by GSK3 controls activity-dependent bulk endocytosis of synaptic vesicles. Nature neuroscience, 13, 845-851.

COLBRAN, R. J. 2004. Protein phosphatases and calcium/calmodulin-dependent protein kinase II-dependent synaptic plasticity. J Neurosci, 24, 8404-9.

COLEMAN, W. L., BILL, C. A. \& BYKHOVSKAIA, M. 2007. Rab3a deletion reduces vesicle docking and transmitter release at the mouse diaphragm synapse. Neuroscience, 148, 1-6.

COPPOLA, T., MAGNiN-LUTHI, S., PERRET-MENOUD, V., GATTESCO, S., SCHIAVO, G. \& REGAZZI, R. 2001. Direct interaction of the Rab3 effector RIM with Ca2+ channels, SNAP-25, and synaptotagmin. J Biol Chem, 276, 32756-62.

CORBIN, J. D. \& KREBS, E. G. 1969. A cyclic AMP - stimulated protein kinase in adipose tissue. Biochemical and Biophysical Research Communications, 36, 328-336.

CORRADINI, E., VALLUR, R., RAAIJMAKERS, L. M., FEIL, S., FEIL, R., HECK, A. J. \& SCHOLTEN, A. 2014. Alterations in the cerebellar (Phospho)proteome of a cyclic guanosine monophosphate (cGMP)-dependent protein kinase knockout mouse. Mol Cell Proteomics, 13, 2004-16.

COUSIN, M. A. \& ROBINSON, P. J. 2001. The dephosphins: dephosphorylation by calcineurin triggers synaptic vesicle endocytosis. Trends in Neurosciences, 24, 659-665. 
COUSIN, M. A., TAN, T. C. \& ROBINSON, P. J. 2001. Protein phosphorylation is required for endocytosis in nerve terminals: potential role for the dephosphins dynamin I and synaptojanin, but not AP180 or amphiphysin. J Neurochem, 76, 105-16.

COX, J., HEIN, M. Y., LUBER, C. A., PARON, I., NAGARAJ, N. \& MANN, M. 2014. Accurate proteome-wide label-free quantification by delayed normalization and maximal peptide ratio extraction, termed MaxLFQ. Mol Cell Proteomics, 13, 2513-26.

COX, J. \& MANN, M. 2008. MaxQuant enables high peptide identification rates, individualized p.p.b.-range mass accuracies and proteome-wide protein quantification. Nat Biotechnol, 26, 1367-72.

COX, J., MATIC, I., HILGER, M., NAGARAJ, N., SELBACH, M., OLSEN, J. V. \& MANN, M. 2009. A practical guide to the MaxQuant computational platform for SILAC-based quantitative proteomics. Nat Protoc, 4, 698-705.

COX, J., NEUHAUSER, N., MICHALSKI, A., SCHELTEMA, R. A., OLSEN, J. V. \& MANN, M. 2011. Andromeda: A Peptide Search Engine Integrated into the MaxQuant Environment. Journal of Proteome Research, 10, 1794-1805.

CRAFT, G. E., GRAHAM, M. E., BACHE, N., LARSEN, M. R. \& ROBINSON, P. J. 2008. The in vivo phosphorylation sites in multiple isoforms of amphiphysin I from rat brain nerve terminals. Mol Cell Proteomics, 7, 1146-61.

DAI, Y., TARU, H., DEKEN, S. L., GRILL, B., ACKLEY, B., NONET, M. L. \& JIN, Y. 2006. SYD-2 Liprin-alpha organizes presynaptic active zone formation through ELKS. Nat Neurosci, 9, 1479-87.

DANIEL, J. A., MALLADI, C. S., KETTLE, E., MCCLUSKEY, A. \& ROBINSON, P. J. 2012. Analysis of synaptic vesicle endocytosis in synaptosomes by high-content screening. Nat. Protocols, 7, 1439-1455.

DAVLETOV, B., SONTAG, J. M., HATA, Y., PETRENKO, A. G., FYKSE, E. M., JAHN, R. \& SUDHOF, T. C. 1993. Phosphorylation of synaptotagmin I by casein kinase II. J Biol Chem, 268, 6816-22.

DAVYDOVA, D., MARINI, C., KING, C., KLUEVA, J., BISCHOF, F., ROMORINI, S., MONTENEGROVENEGAS, C., HEINE, M., SCHNEIDER, R., SCHRODER, M. S., ALTROCK, W. D., HENNEBERGER, C., RUSAKOV, D. A., GUNDELFINGER, E. D. \& FEJTOVA, A. 2014. Bassoon specifically controls presynaptic P/Q-type $\mathrm{Ca}(2+)$ channels via RIM-binding protein. Neuron, 82, 181-94.

DE JONG, A. P., MEIJER, M., SAARLOOS, I., CORNELISSE, L. N., TOONEN, R. F., SORENSEN, J. B. \& VERHAGE, M. 2016. Phosphorylation of synaptotagmin-1 controls a post-priming step in PKC-dependent presynaptic plasticity. Proc Natl Acad Sci U S A, 113, 5095-100.

DE LANGE, R. P., DE ROOS, A. D. \& BORST, J. G. 2003. Two modes of vesicle recycling in the rat calyx of Held. J Neurosci, 23, 10164-73.

DEGTIAR, V. E., SCHELLER, R. H. \& TSIEN, R. W. 2000. Syntaxin modulation of slow inactivation of N-type calcium channels. Journal of Neuroscience, 20, 4355-4367.

DENG, L., KAESER, P. S., XU, W. \& SUDHOF, T. C. 2011. RIM proteins activate vesicle priming by reversing autoinhibitory homodimerization of Munc13. Neuron, 69, 317-31.

DI PAOLO, G., ANTONSSON, B., KASSEL, D., RIEDERER, B. M. \& GRENNINGLOH, G. 1997. Phosphorylation regulates the microtubule-destabilizing activity of stathmin and its interaction with tubulin. FEBS Letters, 416, 149-152.

DITTMAN, J. \& RYAN, T. A. 2009. Molecular circuitry of endocytosis at nerve terminals. Annu Rev Cell Dev Biol, 25, 133-60. 
DOUBLEDAY, P. F. \& BALLIF, B. A. 2014. Developmentally-Dynamic Murine Brain Proteomes and Phosphoproteomes Revealed by Quantitative Proteomics. Proteomes, 2, 197-207.

DOUSSAU, F. \& AUGUSTINE, G. J. 2000. The actin cytoskeleton and neurotransmitter release: An overview. Biochimie, 82, 353-363.

DUAN, L. \& COBB, M. H. 2010. Calcineurin increases glucose activation of ERK1/2 by reversing negative feedback. Proceedings of the National Academy of Sciences of the United States of America, 107, 22314-22319.

DUTIL, E. M., TOKER, A. \& NEWTON, A. C. 1998. Regulation of conventional protein kinase C isozymes by phosphoinositide-dependent kinase 1 (PDK-1). Current Biology, 8, 13661375.

EDMAN, P. 1949. A method for the determination of amino acid sequence in peptides. Arch Biochem, 22, 475.

EISENBERG, E. \& GREENE, L. E. 2007. Multiple roles of auxilin and hsc70 in clathrin-mediated endocytosis. Traffic, 8, 640-6.

EL-ANEED, A., COHEN, A. \& BANOUB, J. 2009. Mass Spectrometry, Review of the Basics: Electrospray, MALDI, and Commonly Used Mass Analyzers. Applied Spectroscopy Reviews, 44, 210-230.

ELDAR-FINKELMAN, H. 2002. Glycogen synthase kinase 3: An emerging therapeutic target. Trends in Molecular Medicine, 8, 126-132.

ELIAS, J. E. \& GYGI, S. P. 2007. Target-decoy search strategy for increased confidence in largescale protein identifications by mass spectrometry. Nat Meth, 4, 207-214.

ELKINS, J. M. \& KNAPP, S. 2012. The Structure of the Full-Length Tetrameric PKA Regulatory RII $\beta$ Complex Reveals the Mechanism of Allosteric PKA Activation. Science Signaling, 5, pe21-pe21.

FASSIO, A., FADDA, M. \& BENFENATI, F. 2016. Molecular Machines Determining the Fate of Endocytosed Synaptic Vesicles in Nerve Terminals. Front Synaptic Neurosci, 8, 10.

FENN, J. B., MANN, M., MENG, C. K., WONG, S. F. \& WHITEHOUSE, C. M. 1989. Electrospray ionization for mass spectrometry of large biomolecules. Science, 246, 64-71.

FERNANDEZ-ALFONSO, T. \& RYAN, T. A. 2008. A heterogeneous "resting" pool of synaptic vesicles that is dynamically interchanged across boutons in mammalian CNS synapses. Brain Cell Biol, 36, 87-100.

FILIOU, M. D., BISLE, B., RECKOW, S., TEPLYTSKA, L., MACCARRONE, G. \& TURCK, C. W. 2010. Profiling of mouse synaptosome proteome and phosphoproteome by IEF. Electrophoresis, 31, 1294-301.

FIOL, C. J., WANG, A., ROESKE, R. W. \& ROACH, P. J. 1990. Ordered multisite protein phosphorylation. Analysis of glycogen synthase kinase 3 action using model peptide substrates. J Biol Chem, 265, 6061-5.

FIORAVANTE, D., CHU, Y., MYOGA, M. H., LEITGES, M. \& REGEHR, W. G. 2011. Calciumdependent isoforms of protein kinase $\mathrm{C}$ mediate posttetanic potentiation at the calyx of Held. Neuron, 70, 1005-19.

FISCHER VON MOLLARD, G., SUDHOF, T. C. \& JAHN, R. 1991. A small GTP-binding protein dissociates from synaptic vesicles during exocytosis. Nature, 349, 79-81.

FLETCHER, A. I., SHUANG, R., GIOVANNUCCI, D. R., ZHANG, L., BITTNER, M. A. \& STUENKEL, E. L. 1999. Regulation of exocytosis by cyclin-dependent kinase 5 via phosphorylation of Munc18. J Biol Chem, 274, 4027-35.

FLINT, A., PALADINI, R. \& KOSHLAND, D. 1990. Autophosphorylation of protein kinase C at three separated regions of its primary sequence. Science, 249, 408-411. 
FLINT, A. J., TIGANIS, T., BARFORD, D. \& TONKS, N. K. 1997. Development of "substratetrapping" mutants to identify physiological substrates of protein tyrosine phosphatases. Proceedings of the National Academy of Sciences of the United States of America, 94, 1680-1685.

FUJIMOTO, L. M., ROTH, R., HEUSER, J. E. \& SCHMID, S. L. 2000. Actin assembly plays a variable, but not obligatory role in receptor-mediated endocytosis in mammalian cells. Traffic, 1, 161-71.

FUKUDA, M. 2003. Distinct Rab binding specificity of Rim1, Rim2, rabphilin, and Noc2. Identification of a critical determinant of Rab3A/Rab27A recognition by Rim2. J Biol Chem, 278, 15373-80.

FUKUNAGA, K., MULLER, D., OHMITSU, M., BAKO, E., DEPAOLI-ROACH, A. A. \& MIYAMOTO, E. 2000. Decreased protein phosphatase $2 \mathrm{~A}$ activity in hippocampal long-term potentiation. J Neurochem, 74, 807-17.

GAIDAROV, I., SANTINI, F., WARREN, R. A. \& KEEN, J. H. 1999. Spatial control of coated-pit dynamics in living cells. Nat Cell Biol, 1, 1-7.

GAO, J., HIRATA, M., MIZOKAMI, A., ZHAO, J., TAKAHASHI, I., TAKEUCHI, H. \& HIRATA, M. 2016. Differential role of SNAP-25 phosphorylation by protein kinases A and C in the regulation of SNARE complex formation and exocytosis in PC12 cells. Cell Signal, 28, 425-37.

GENESCA, L., AUBAREDA, A., FUENTES, J. J., ESTIVILL, X., DE LA LUNA, S. \& PEREZ-RIBA, M. 2003. Phosphorylation of calcipressin 1 increases its ability to inhibit calcineurin and decreases calcipressin half-life. Biochem J, 374, 567-75.

GIANSANTI, P., TSIATSIANI, L., LOW, T. Y. \& HECK, A. J. R. 2016. Six alternative proteases for mass spectrometry-based proteomics beyond trypsin. Nat. Protocols, 11, 993-1006.

GIRAULT, J. A., HEMMINGS, H. C., JR., WILLIAMS, K. R., NAIRN, A. C. \& GREENGARD, P. 1989. Phosphorylation of DARPP-32, a dopamine- and cAMP-regulated phosphoprotein, by casein kinase II. J Biol Chem, 264, 21748-59.

GITLER, D., TAKAGISHI, Y., FENG, J., REN, Y., RODRIGUIZ, R. M., WETSEL, W. C., GREENGARD, P. \& AUGUSTINE, G. J. 2004. Different presynaptic roles of synapsins at excitatory and inhibitory synapses. J Neurosci, 24, 11368-80.

GLISH, G. L. \& BURINSKY, D. J. 2008. Hybrid mass spectrometers for tandem mass spectrometry. J Am Soc Mass Spectrom, 19, 161-72.

GOSWAMI, T., LI, X., SMITH, A. M., LUDEROWSKI, E. M., VINCENT, J. J., RUSH, J. \& BALLIF, B. A. 2012. Comparative phosphoproteomic analysis of neonatal and adult murine brain. Proteomics, 12, 2185-9.

GRAHAM, M. E., ANGGONO, V., BACHE, N., LARSEN, M. R., CRAFT, G. E. \& ROBINSON, P. J. 2007. The in vivo phosphorylation sites of rat brain dynamin I. J Biol Chem, 282, 14695-707.

GREENGARD, P. 2001. The neurobiology of slow synaptic transmission. Science, 294, 102430.

GREENGARD, P., ALLEN, P. B. \& NAIRN, A. C. 1999. Beyond the Dopamine Receptor: the DARPP-32/Protein Phosphatase-1 Cascade. Neuron, 23, 435-447.

GRIMSRUD, P. A., SWANEY, D. L., WENGER, C. D., BEAUCHENE, N. A. \& COON, J. J. 2010. Phosphoproteomics for the Masses. ACS Chemical Biology, 5, 105-119.

GUNDELFINGER, E. D., REISSNER, C. \& GARNER, C. C. 2015. Role of Bassoon and Piccolo in Assembly and Molecular Organization of the Active Zone. Frontiers in Synaptic Neuroscience, 7, 19. 
GUTHALS, A. \& BANDEIRA, N. 2012. Peptide Identification by Tandem Mass Spectrometry with Alternate Fragmentation Modes. Molecular \& Cellular Proteomics, 11, 550-557.

HALLERMANN, S., FEJTOVA, A., SCHMIDT, H., WEYHERSMULLER, A., SILVER, R. A., GUNDELFINGER, E. D. \& EILERS, J. 2010. Bassoon speeds vesicle reloading at a central excitatory synapse. Neuron, $68,710-23$.

HALLS, M. L. \& COOPER, D. M. F. 2011. Regulation by Ca(2+)-Signaling Pathways of Adenylyl Cyclases. Cold Spring Harbor Perspectives in Biology, 3, a004143.

HAN, Y., KAESER, P. S., SUDHOF, T. C. \& SCHNEGGENBURGER, R. 2011. RIM determines $\mathrm{Ca}(2)+$ channel density and vesicle docking at the presynaptic active zone. Neuron, 69, 304-16.

HANSE, E. \& GUSTAFSSON, B. 2001. Vesicle release probability and pre-primed pool at glutamatergic synapses in area CA1 of the rat neonatal hippocampus. J Physiol, 531, 481-93.

HANSON, P. I. \& SCHULMAN, H. 1992. Neuronal Ca2+/calmodulin-dependent protein kinases. Annu Rev Biochem, 61, 559-601.

HAO, W., LUO, Z., ZHENG, L., PRASAD, K. \& LAFER, E. M. 1999. AP180 and AP-2 interact directly in a complex that cooperatively assembles clathrin. J Biol Chem, 274, 2278594.

HARATA, N., PYLE, J. L., ARAVANIS, A. M., MOZHAYEVA, M., KAVALALI, E. T. \& TSIEN, R. W. 2001. Limited numbers of recycling vesicles in small CNS nerve terminals: implications for neural signaling and vesicular cycling. Trends Neurosci, 24, 637-43.

HAUCKE, V., NEHER, E. \& SIGRIST, S. J. 2011. Protein scaffolds in the coupling of synaptic exocytosis and endocytosis. Nat Rev Neurosci, 12, 127-138.

HAYASHI, M., RAIMONDI, A., O'TOOLE, E., PARADISE, S., COLlESI, C., CREMONA, O., FERGUSON, S. M. \& DE CAMILLI, P. 2008. Cell- and stimulus-dependent heterogeneity of synaptic vesicle endocytic recycling mechanisms revealed by studies of dynamin 1null neurons. Proc Natl Acad Sci US A, 105, 2175-80.

HEEMSKERK, J. W. M., HARPER, M. T., COSEMANS, J. M. E. M. \& POOLE, A. W. 2011. Unravelling the different functions of protein kinase C isoforms in platelets. FEBS Letters, 585, 1711-1716.

HELD, R. G., LIU, C. \& KAESER, P. S. 2016. ELKS controls the pool of readily releasable vesicles at excitatory synapses through its N-terminal coiled-coil domains. Elife, 5.

HERNANDEZ, F., LANGA, E., CUADROS, R., AVILA, J. \& VILLANUEVA, N. 2010. Regulation of GSK3 isoforms by phosphatases PP1 and PP2A. Mol Cell Biochem, 344, 211-5.

HERNÁNDEZ, F., LANGA, E., CUADROS, R., AVILA, J. \& VILLANUEVA, N. 2010. Regulation of GSK3 isoforms by phosphatases PP1 and PP2A. Molecular and Cellular Biochemistry, 344, 211-215.

HILFIKER, S., PIERIBONE, V. A., NORDSTEDT, C., GREENGARD, P. \& CZERNIK, A. J. 1999. Regulation of synaptotagmin I phosphorylation by multiple protein kinases. $J$ Neurochem, 73, 921-32.

HIRLING, H. \& SCHELLER, R. H. 1996. Phosphorylation of synaptic vesicle proteins: modulation of the alpha SNAP interaction with the core complex. Proc Natl Acad Sci U $S A, 93,11945-9$.

HOLMGREN, A., BOUHY, D. \& TIMMERMAN, V. 2012. Neurofilament phosphorylation and their proline-directed kinases in health and disease. Journal of the Peripheral Nervous System, 17, 365-376. 
HORVATH, C. A. J., VANDEN BROECK, D., BOULET, G. A. V., BOGERS, J. \& DE WOLF, M. J. S. 2007. Epsin: Inducing membrane curvature. The International Journal of Biochemistry \& Cell Biology, 39, 1765-1770.

HSU, J. L., HUANG, S. Y., CHOW, N. H. \& CHEN, S. H. 2003. Stable-isotope dimethyl labeling for quantitative proteomics. Anal Chem, 75, 6843-52.

HUANG, F. L. \& GLINSMANN, W. H. 1975. Inactivation of rabbit muscle phosphorylase phosphatase by cyclic AMP-dependent kinas. Proc Natl Acad Sci U S A, 72, 3004-8.

HUNTER, T. 2007. The age of crosstalk: phosphorylation, ubiquitination, and beyond. Mol Cell, 28, 730-8.

HUNTER, T. 2014. The genesis of tyrosine phosphorylation. Cold Spring Harb Perspect Biol, 6, a020644.

IGARASHI, M. \& WATANABE, M. 2007. Roles of calmodulin and calmodulin-binding proteins in synaptic vesicle recycling during regulated exocytosis at submicromolar $\mathrm{Ca} 2+$ concentrations. Neurosci Res, 58, 226-33.

ITOH, T. \& DE CAMILLI, P. 2006. BAR, F-BAR (EFC) and ENTH/ANTH domains in the regulation of membrane-cytosol interfaces and membrane curvature. Biochim Biophys Acta, 1761, 897-912.

JAHN, R. \& FASSHAUER, D. 2012. Molecular machines governing exocytosis of synaptic vesicles. Nature, 490, 201-7.

JARVIS, S. E. \& ZAMPONI, G. W. 2001. Distinct molecular determinants govern syntaxin 1Amediated inactivation and G-protein inhibition of N-type calcium channels. Journal of Neuroscience, 21, 2939-2948.

JEDRYCHOWSKI, M. P., HUTTLIN, E. L., HAAS, W., SOWA, M. E., RAD, R. \& GYGI, S. P. 2011. Evaluation of HCD- and CID-type Fragmentation Within Their Respective Detection Platforms For Murine Phosphoproteomics. Molecular \& Cellular Proteomics : MCP, 10, M111.009910.

JI, C. \& LI, L. 2005. Quantitative Proteome Analysis Using Differential Stable Isotopic Labeling and Microbore LC-MALDI MS and MS/MS. Journal of Proteome Research, 4, 734-742.

JOVANOVIC, J. N., CZERNIK, A. J., FIENBERG, A. A., GREENGARD, P. \& SIHRA, T. S. 2000. Synapsins as mediators of BDNF-enhanced neurotransmitter release. Nat Neurosci, 3, 323-9.

JOVANOVIC, J. N., SIHRA, T. S., NAIRN, A. C., HEMMINGS, H. C., JR., GREENGARD, P. \& CZERNIK, A. J. 2001. Opposing changes in phosphorylation of specific sites in synapsin I during Ca2+-dependent glutamate release in isolated nerve terminals. J Neurosci, 21, 794453.

JUANES-GARCIA, A., CHAPMAN, J. R., AGUILAR-CUENCA, R., DELGADO-AREVALO, C., HODGES, J., WHITMORE, L. A., SHABANOWITZ, J., HUNT, D. F., HORWITZ, A. R. \& VICENTE-MANZANARES, M. 2015. A regulatory motif in nonmuscle myosin II-B regulates its role in migratory front-back polarity. The Journal of Cell Biology, 209, 2332.

JUNG, N. \& HAUCKE, V. 2007. Clathrin-mediated endocytosis at synapses. Traffic, 8, 1129-36. KADAMUR, G. \& ROSS, E. M. 2013. Mammalian phospholipase C. Annu Rev Physiol, 75, $127-$ 54.

KAESER, P. S., DENG, L., CHAVEZ, A. E., LIU, X., CASTILlO, P. E. \& SUDHOF, T. C. 2009. ELKS2alpha/CAST deletion selectively increases neurotransmitter release at inhibitory synapses. Neuron, 64, 227-39. 
KAESER, P. S., DENG, L., WANG, Y., DULUBOVA, I., LIU, X., RIZO, J. \& SUDHOF, T. C. 2011a. RIM proteins tether $\mathrm{Ca} 2+$ channels to presynaptic active zones via a direct PDZ-domain interaction. Cell, 144, 282-95.

KAESER, P. S., DENG, L., WANG, Y., DULUBOVA, I., LIU, X., RIZO, J. \& SÜDHOF, T. C. 2011b. RIM proteins tether $\mathrm{Ca}(2+)$-channels to presynaptic active zones via a direct PDZ-domain interaction. Cell, 144, 282-295.

KAESER, P. S., KWON, H.-B., BLUNDELL, J., CHEVALEYRE, V., MORISHITA, W., MALENKA, R. C., POWELL, C. M., CASTILLO, P. E. \& SÜDHOF, T. C. 2008. RIM1 $\alpha$ phosphorylation at serine-413 by protein kinase $\mathrm{A}$ is not required for presynaptic long-term plasticity or learning. Proceedings of the National Academy of Sciences, 105, 14680-14685.

KAMEI, H., SAITO, T., OZAWA, M., FUJITA, Y., ASADA, A., BIBB, J. A., SAIDO, T. C., SORIMACHI, H. \& HISANAGA, S.-I. 2007. Suppression of Calpain-dependent Cleavage of the CDK5 Activator p35 to p25 by Site-specific Phosphorylation. Journal of Biological Chemistry, $282,1687-1694$.

KELSTRUP, C. D., JERSIE-CHRISTENSEN, R. R., BATTH, T. S., ARREY, T. N., KUEHN, A., KELLMANN, M. \& OLSEN, J. V. 2014. Rapid and deep proteomes by faster sequencing on a benchtop quadrupole ultra-high-field Orbitrap mass spectrometer. J Proteome Res, 13, 6187-95.

KENNEDY, M. B. \& GREENGARD, P. 1981. Two calcium/calmodulin-dependent protein kinases, which are highly concentrated in brain, phosphorylate protein I at distinct sites. Proc Natl Acad Sci U S A, 78, 1293-7.

KESAVAPANY, S., LI, B. S. \& PANT, H. C. 2003. Cyclin-dependent kinase 5 in neurofilament function and regulation. Neurosignals, 12, 252-64.

KIM, K., LAKHANPAL, G., LU, H. E., KHAN, M., SUZUKI, A., HAYASHI, M. K., NARAYANAN, R., LUYBEN, T. T., MATSUDA, T., NAGAI, T., BLANPIED, T. A., HAYASHI, Y. \& OKAMOTO, K. 2015. A Temporary Gating of Actin Remodeling during Synaptic Plasticity Consists of the Interplay between the Kinase and Structural Functions of CaMKII. Neuron, 87, 81326.

KIM, S. H. \& RYAN, T. A. 2010. CDK5 serves as a major control point in neurotransmitter release. Neuron, 67, 797-809.

KIM, S. H. \& RYAN, T. A. 2013. Balance of calcineurin Aalpha and CDK5 activities sets release probability at nerve terminals. J Neurosci, 33, 8937-50.

KIM, Y., LEE, Y. I., SEO, M., KIM, S. Y., LEE, J. E., YOUN, H. D., KIM, Y. S. \& JUHNN, Y. S. 2009. Calcineurin dephosphorylates glycogen synthase kinase-3 beta at serine- 9 in neuroblast-derived cells. J Neurochem, 111, 344-54.

KOHANSAL-NODEHI, M., CHUA, J. J., URLAUB, H., JAHN, R. \& CZERNIK, D. 2016. Analysis of protein phosphorylation in nerve terminal reveals extensive changes in active zone proteins upon exocytosis. Elife, 5.

KONERMANN, L., AHADI, E., RODRIGUEZ, A. D. \& VAHIDI, S. 2013. Unraveling the Mechanism of Electrospray Ionization. Analytical Chemistry, 85, 2-9.

KORBER, C., HORSTMANN, H., VENKATARAMANI, V., HERRMANNSDORFER, F., KREMER, T., KAISER, M., SCHWENGER, D. B., AHMED, S., DEAN, C., DRESBACH, T. \& KUNER, T. 2015. Modulation of Presynaptic Release Probability by the Vertebrate-Specific Protein Mover. Neuron, 87, 521-33.

KOVANICH, D., CAPPADONA, S., RAIJMAKERS, R., MOHAMMED, S., SCHOLTEN, A. \& HECK, A. J. R. 2012. Applications of stable isotope dimethyl labeling in quantitative proteomics. Analytical and Bioanalytical Chemistry, 404, 991-1009. 
KREMER, T., KEMPF, C., WITTENMAYER, N., NAWROTZKI, R., KUNER, T., KIRSCH, J. \& DRESBACH, T. 2007. Mover is a novel vertebrate-specific presynaptic protein with differential distribution at subsets of CNS synapses. FEBS Lett, 581, 4727-33.

KRÜGER, M., MOSER, M., USSAR, S., THIEVESSEN, I., LUBER, C. A., FORNER, F., SCHMIDT, S., ZANIVAN, S., FÄSSLER, R. \& MANN, M. 2008. SILAC Mouse for Quantitative Proteomics Uncovers Kindlin-3 as an Essential Factor for Red Blood Cell Function. Cell, 134, 353364.

KUMMEL, D., KRISHNAKUMAR, S. S., RADOFF, D. T., LI, F., GIRAUDO, C. G., PINCET, F., ROTHMAN, J. E. \& REINISCH, K. M. 2011. Complexin cross-links prefusion SNAREs into a zigzag array. Nat Struct Mol Biol, 18, 927-33.

KUO, S. W. \& DODD, P. R. 2011. Electrically evoked synaptosomal amino acid transmitter release in human brain in alcohol misuse. Neurosignals, 19, 117-27.

KUROMI, H. \& KIDOKORO, Y. 2000. Tetanic stimulation recruits vesicles from reserve pool via a cAMP-mediated process in Drosophila synapses. Neuron, 27, 133-43.

KUROMI, H. \& KIDOKORO, Y. 2003. Two synaptic vesicle pools, vesicle recruitment and replenishment of pools at the Drosophila neuromuscular junction. J Neurocytol, 32, 551-65.

LAMAZE, C., FUJIMOTO, L. M., YIN, H. L. \& SCHMID, S. L. 1997. The actin cytoskeleton is required for receptor-mediated endocytosis in mammalian cells. J Biol Chem, 272, 20332-5.

LARSEN, M. R., THINGHOLM, T. E., JENSEN, O. N., ROEPSTORFF, P. \& JORGENSEN, T. J. 2005. Highly selective enrichment of phosphorylated peptides from peptide mixtures using titanium dioxide microcolumns. Mol Cell Proteomics, 4, 873-86.

LE GOUVELLO, S., MANCEAU, V. \& SOBEL, A. 1998. Serine 16 of stathmin as a cytosolic target for $\mathrm{Ca} 2+/$ calmodulin-dependent kinase II after CD2 triggering of human $\mathrm{T}$ lymphocytes. J Immunol, 161, 1113-22.

LEE, S. Y., WENK, M. R., KIM, Y., NAIRN, A. C. \& DE CAMILLI, P. 2004. Regulation of synaptojanin 1 by cyclin-dependent kinase 5 at synapses. Proceedings of the National Academy of Sciences of the United States of America, 101, 546-551.

LEENDERS, A. G., SCHOLTEN, G., WIEGANT, V. M., DA SILVA, F. H. \& GHIJSEN, W. E. 1999. Activity-dependent neurotransmitter release kinetics: correlation with changes in morphological distributions of small and large vesicles in central nerve terminals. Eur J Neurosci, 11, 4269-77.

LEITE, S. C., SAMPAIO, P., SOUSA, V. F., NOGUEIRA-RODRIGUES, J., PINTO-COSTA, R., PETERS, L. L., BRITES, P. \& SOUSA, M. M. 2016. The actin-binding protein $\alpha$-adducin is required for maintaining axon diameter. Cell reports, 15, 490-498.

LEMEER, S. \& HECK, A. J. 2009. The phosphoproteomics data explosion. Curr Opin Chem Biol, $13,414-20$.

LENGYEL, I., FIEUW-MAKAROFF, S., HALL, A. L., SIM, A. T., ROSTAS, J. A. \& DUNKLEY, P. R. 2000. Modulation of the phosphorylation and activity of calcium/calmodulindependent protein kinase II by zinc. J Neurochem, 75, 594-605.

LIANG, S., WEI, F. Y., WU, Y. M., TANABE, K., ABE, T., ODA, Y., YOSHIDA, Y., YAMADA, H., MATSUI, H., TOMIZAWA, K. \& TAKEI, K. 2007. Major Cdk5-dependent phosphorylation sites of amphiphysin 1 are implicated in the regulation of the membrane binding and endocytosis. J Neurochem, 102, 1466-76. 
LIM, S. H., MOON, J., LEE, M. \& LEE, J. R. 2013. PTPRT regulates the interaction of Syntaxinbinding protein 1 with Syntaxin 1 through dephosphorylation of specific tyrosine residue. Biochem Biophys Res Commun, 439, 40-6.

LISMAN, J., SCHULMAN, H. \& CLINE, H. 2002. The molecular basis of CaMKII function in synaptic and behavioural memory. Nat Rev Neurosci, 3, 175-90.

LIU, H., SADYGOV, R. G. \& YATES, J. R., 3RD 2004. A model for random sampling and estimation of relative protein abundance in shotgun proteomics. Anal Chem, 76, 4193201.

LIU, J. P., POWELL, K. A., SUDHOF, T. C. \& ROBINSON, P. J. 1994. Dynamin I is a Ca(2+)sensitive phospholipid-binding protein with very high affinity for protein kinase C. $J$ Biol Chem, 269, 21043-50.

LIU, Z. Q., MAHMOOD, T. \& YANG, P. C. 2014. Western blot: technique, theory and trouble shooting. N Am J Med Sci, 6, 160.

LIZCANO, J. M., GORANSSON, O., TOTH, R., DEAK, M., MORRICE, N. A., BOUDEAU, J., HAWLEY, S. A., UDD, L., MAKELA, T. P., HARDIE, D. G. \& ALESSI, D. R. 2004. LKB1 is a master kinase that activates 13 kinases of the AMPK subfamily, including MARK/PAR-1. Embo $j, 23,833-43$.

LLINAS, R., GRUNER, J. A., SUGIMORI, M., MCGUINNESS, T. L. \& GREENGARD, P. 1991. Regulation by synapsin I and $\mathrm{Ca}(2+)$-calmodulin-dependent protein kinase II of the transmitter release in squid giant synapse.J Physiol, 436, 257-82.

LLINAS, R., MCGUINNESS, T. L., LEONARD, C. S., SUGIMORI, M. \& GREENGARD, P. 1985. Intraterminal injection of synapsin I or calcium/calmodulin-dependent protein kinase II alters neurotransmitter release at the squid giant synapse. Proc Natl Acad Sci U S A, 82, 3035-9.

LOLLI, G., PINNA, L. A. \& BATTISTUTTA, R. 2012. Structural determinants of protein kinase CK2 regulation by autoinhibitory polymerization. ACS Chem Biol, 7, 1158-63.

LONART, G., SCHOCH, S., KAESER, P. S., LARKIN, C. J., SÜDHOF, T. C. \& LINDEN, D. J. 2003. Phosphorylation of RIM1 $\alpha$ by PKA Triggers Presynaptic Long-Term Potentiation at Cerebellar Parallel Fiber Synapses. Cell, 115, 49-60.

LU, I. C., LEE, C., LEE, Y. T. \& NI, C. K. 2015. Ionization Mechanism of Matrix-Assisted Laser Desorption/Ionization. Annu Rev Anal Chem (Palo Alto Calif), 8, 21-39.

LUDWIG, K. R., SUN, L., ZHU, G., DOVICHI, N. J. \& HUMMON, A. B. 2015. Over 2300 Phosphorylated Peptide Identifications with Single-Shot Capillary Zone Electrophoresis-Tandem Mass Spectrometry in a 100 min Separation. Anal Chem, 87, 9532-7.

MA, C., SU, L., SEVEN, A. B., XU, Y. \& RIZO, J. 2013. Reconstitution of the vital functions of Munc18 and Munc13 in neurotransmitter release. Science, 339, 421-5.

MA, L., TANG, H., REN, Y., DENG, H., WU, J. \& WANG, Z. 2012. p38alpha MAP kinase phosphorylates RCAN1 and regulates its interaction with calcineurin. Sci China Life Sci, 55, 559-66.

MACEK, B., MANN, M. \& OLSEN, J. V. 2009. Global and site-specific quantitative phosphoproteomics: principles and applications. Annu Rev Pharmacol Toxicol, 49, 199-221.

MAGUPALLI, V. G., MOCHIDA, S., YAN, J., JIANG, X., WESTENBROEK, R. E., NAIRN, A. C., SCHEUER, T. \& CATTERALL, W. A. 2013. Ca2+-independent activation of $\mathrm{Ca} 2+/$ calmodulin-dependent protein kinase II bound to the C-terminal domain of CaV2.1 calcium channels. J Biol Chem, 288, 4637-48. 
MAKAROV, A., DENISOV, E. \& LANGE, 0. 2009. Performance evaluation of a high-field Orbitrap mass analyzer. J Am Soc Mass Spectrom, 20, 1391-6.

MALLICK, P. \& KUSTER, B. 2010. Proteomics: a pragmatic perspective. Nat Biotech, 28, 695709.

MALSAM, J., SEILER, F., SCHOLLMEIER, Y., RUSU, P., KRAUSE, J. M. \& SÖLLNER, T. H. 2009. The carboxy-terminal domain of complexin I stimulates liposome fusion. Proceedings of the National Academy of Sciences of the United States of America, 106, 2001-2006.

MANNING, G., WHYTE, D. B., MARTINEZ, R., HUNTER, T. \& SUDARSANAM, S. 2002. The protein kinase complement of the human genome. Science, 298, 1912-34.

MARKS, B. \& MCMAHON, H. T. 1998. Calcium triggers calcineurin-dependent synaptic vesicle recycling in mammalian nerve terminals. Curr Biol, 8, 740-9.

MARX, H., LEMEER, S., SCHLIEP, J. E., MATHERON, L., MOHAMMED, S., COX, J., MANN, M., HECK, A. J. R. \& KUSTER, B. 2013. A large synthetic peptide and phosphopeptide reference library for mass spectrometry-based proteomics. Nat Biotech, 31, 557-564.

MATVEEVA, E. A., WHITEHEART, S. W., VANAMAN, T. C. \& SLEVIN, J. T. 2001. Phosphorylation of the N-ethylmaleimide-sensitive factor is associated with depolarization-dependent neurotransmitter release from synaptosomes. J Biol Chem, 276, 12174-81.

MCAVOY, T., ALLEN, P. B., OBAISHI, H., NAKANISHI, H., TAKAI, Y., GREENGARD, P., NAIRN, A. C. \& HEMMINGS, H. C., JR. 1999. Regulation of neurabin I interaction with protein phosphatase 1 by phosphorylation. Biochemistry, 38, 12943-9.

MCMAHON, H. T. \& GALLOP, J. L. 2005. Membrane curvature and mechanisms of dynamic cell membrane remodelling. Nature, 438, 590-6.

MCMAHON, H. T. \& NICHOLLS, D. G. 1991. Transmitter glutamate release from isolated nerve terminals: evidence for biphasic release and triggering by localized Ca2+. J Neurochem, 56, 86-94.

MEDINA, M. \& WANDOSELL, F. 2011. Deconstructing GSK-3: The Fine Regulation of Its Activity. International Journal of Alzheimer\&\#x2019;s Disease, 2011, 12.

MEDZIHRADSZKY, K. F. \& CHALKLEY, R. J. 2015. LESSONS IN DE NOVO PEPTIDE SEQUENCING BY TANDEM MASS SPECTROMETRY. Mass spectrometry reviews, 34, 4363.

MEINRENKEN, C. J., BORST, J. G. \& SAKMANN, B. 2002. Calcium secretion coupling at calyx of Held governed by nonuniform channel-vesicle topography. J Neurosci, 22, 1648-67.

MENDOZA SCHULZ, A., JING, Z., MARÍA SÁNCHEZ CARO, J., WETZEL, F., DRESBACH, T., STRENZKE, N., WICHMANN, C. \& MOSER, T. 2014. Bassoon-disruption slows vesicle replenishment and induces homeostatic plasticity at a CNS synapse. The EMBO Journal, 33, 512-527.

MILLAN, C., TORRES, M. \& SANCHEZ-PRIETO, J. 2003. Co-activation of PKA and PKC in cerebrocortical nerve terminals synergistically facilitates glutamate release. $J$ Neurochem, 87, 1101-11.

MILLER, S. G. \& KENNEDY, M. B. 1986. Regulation of brain type II Ca2+/calmodulindependent protein kinase by autophosphorylation: a Ca2+-triggered molecular switch. Cell, 44, 861-70.

MOORE, M. J., KANTER, J. R., JONES, K. C. \& TAYLOR, S. S. 2002. Phosphorylation of the catalytic subunit of protein kinase A. Autophosphorylation versus phosphorylation by phosphoinositide-dependent kinase-1.J Biol Chem, 277, 47878-84.

MOZHAYEVA, M. G., SARA, Y., LIU, X. \& KAVALALI, E. T. 2002. Development of vesicle pools during maturation of hippocampal synapses. J Neurosci, 22, 654-65. 
MUNTON, R. P., TWEEDIE-CULLEN, R., LIVINGSTONE-ZATCHEJ, M., WEINANDY, F., WAIDELICH, M., LONGO, D., GEHRIG, P., POTTHAST, F., RUTISHAUSER, D., GERRITS, B., PANSE, C., SCHLAPBACH, R. \& MANSUY, I. M. 2007. Qualitative and quantitative analyses of protein phosphorylation in naive and stimulated mouse synaptosomal preparations. Mol Cell Proteomics, 6, 283-93.

MURTHY, V. N. \& STEVENS, C. F. 1999. Reversal of synaptic vesicle docking at central synapses. Nat Neurosci, 2, 503-7.

NAGARAJ, N., D'SOUZA, R. C. J., COX, J., OLSEN, J. V. \& MANN, M. 2010. Feasibility of LargeScale Phosphoproteomics with Higher Energy Collisional Dissociation Fragmentation. Journal of Proteome Research, 9, 6786-6794.

NAGY, G., MATTI, U., NEHRING, R. B., BINZ, T., RETTIG, J., NEHER, E. \& SORENSEN, J. B. 2002. Protein kinase C-dependent phosphorylation of synaptosome-associated protein of $25 \mathrm{kDa}$ at Ser187 potentiates vesicle recruitment. J Neurosci, 22, 9278-86.

NAIRN, A. C. \& ADEREM, A. 1992. Calmodulin and protein kinase C cross-talk: the MARCKS protein is an actin filament and plasma membrane cross-linking protein regulated by protein kinase C phosphorylation and by calmodulin. Ciba Found Symp, 164, 145-54; discussion 154-61.

NAKANISHI, H., BREWER, K. A. \& EXTON, J. H. 1993. Activation of the zeta isozyme of protein kinase $\mathrm{C}$ by phosphatidylinositol 3,4,5-trisphosphate. J Biol Chem, 268, 13-6.

NEHER, E. \& SAKABA, T. 2008. Multiple roles of calcium ions in the regulation of neurotransmitter release. Neuron, 59, 861-72.

NGUYEN, T. H., QIU, X., SUN, J. \& MEUNIER, F. A. 2014. Bulk endocytosis at neuronal synapses. Sci China Life Sci, 57, 378-83.

NICHOLLS, D. G. \& SIHRA, T. S. 1986. Synaptosomes possess an exocytotic pool of glutamate. Nature, 321, 772-3.

NICHOLSON-FISH, J. C., KOKOTOS, A. C., GILLINGWATER, T. H., SMILLIE, K. J. \& COUSIN, M. A. 2015. VAMP4 Is an Essential Cargo Molecule for Activity-Dependent Bulk Endocytosis. Neuron, 88, 973-84.

NIELANDER, H. B., ONOFRI, F., VALTORTA, F., SCHIAVO, G., MONTECUCCO, C., GREENGARD, P. \& BENFENATI, F. 1995. Phosphorylation of VAMP/synaptobrevin in synaptic vesicles by endogenous protein kinases. J Neurochem, 65, 1712-20.

NIKOLOV, M., SCHMIDT, C. \& URLAUB, H. 2012. Quantitative mass spectrometry-based proteomics: an overview. Methods Mol Biol, 893, 85-100.

NILSSON, C. L. 2012. Advances in quantitative phosphoproteomics. Anal Chem, 84, 735-46.

NINAN, I. \& ARANCIO, 0. 2004. Presynaptic CaMKII is necessary for synaptic plasticity in cultured hippocampal neurons. Neuron, 42, 129-41.

NISHIMUNE, H., NUMATA, T., CHEN, J., AOKI, Y., WANG, Y., STARR, M. P., MORI, Y. \& STANFORD, J. A. 2012. Active Zone Protein Bassoon Co-Localizes with Presynaptic Calcium Channel, Modifies Channel Function, and Recovers from Aging Related Loss by Exercise. PLOS ONE, 7, e38029.

NIZAMI, S., LEE, V. W., DAVIES, J., LONG, P., JOVANOVIC, J. N. \& SIHRA, T. S. 2010. Presynaptic roles of intracellular $\mathrm{Ca}(2+)$ stores in signalling and exocytosis. Biochem Soc Trans, 38, 529-35.

NORRIS, C. M., BLALOCK, E. M., CHEN, K. C., PORTER, N. M. \& LANDFIELD, P. W. 2002. Calcineurin enhances L-type $\mathrm{Ca}(2+)$ channel activity in hippocampal neurons: increased effect with age in culture. Neuroscience, 110, 213-25. 
OHTSUKA, T., TAKAO-RIKITSU, E., INOUE, E., INOUE, M., TAKEUCHI, M., MATSUBARA, K., DEGUCHI-TAWARADA, M., SATOH, K., MORIMOTO, K., NAKANISHI, H. \& TAKAI, Y. 2002. Cast: a novel protein of the cytomatrix at the active zone of synapses that forms a ternary complex with RIM1 and munc13-1.J Cell Biol, 158, 577-90.

OLSEN, J. V., BLAGOEV, B., GNAD, F., MACEK, B., KUMAR, C., MORTENSEN, P. \& MANN, M. 2006a. Global, in vivo, and site-specific phosphorylation dynamics in signaling networks. Cell, 127, 635-48.

OLSEN, J. V., BLAGOEV, B., GNAD, F., MACEK, B., KUMAR, C., MORTENSEN, P. \& MANN, M. 2006b. Global, In Vivo, and Site-Specific Phosphorylation Dynamics in Signaling Networks. Cell, 127, 635-648.

OLSEN, J. V. \& MANN, M. 2013. Status of large-scale analysis of post-translational modifications by mass spectrometry. Mol Cell Proteomics, 12, 3444-52.

OLSEN, J. V., ONG, S. E. \& MANN, M. 2004. Trypsin cleaves exclusively C-terminal to arginine and lysine residues. Mol Cell Proteomics, 3, 608-14.

ONG, S. E., BLAGOEV, B., KRATCHMAROVA, I., KRISTENSEN, D. B., STEEN, H., PANDEY, A. \& MANN, M. 2002. Stable isotope labeling by amino acids in cell culture, SILAC, as a simple and accurate approach to expression proteomics. Mol Cell Proteomics, 1, 37686.

ONOFRI, F., MESSA, M., MATAFORA, V., BONANNO, G., CORRADI, A., BACHI, A., VALTORTA, F. \& BENFENATI, F. 2007. Synapsin phosphorylation by SRC tyrosine kinase enhances SRC activity in synaptic vesicles. J Biol Chem, 282, 15754-67.

OPAZO, F., PUNGE, A., BUCKERS, J., HOOPMANN, P., KASTRUP, L., HELL, S. W. \& RIZZOLI, S. O. 2010. Limited intermixing of synaptic vesicle components upon vesicle recycling. Traffic, 11, 800-12.

PALMISANO, G., PARKER, B. L., ENGHOLM-KELLER, K., LENDAL, S. E., KULEJ, K., SCHULZ, M., SCHWAMMLE, V., GRAHAM, M. E., SAXTORPH, H., CORDWELL, S. J. \& LARSEN, M. R. 2012. A novel method for the simultaneous enrichment, identification, and quantification of phosphopeptides and sialylated glycopeptides applied to a temporal profile of mouse brain development. Mol Cell Proteomics, 11, 1191-202.

PANT, H. C. \& VEERANNA 1995. Neurofilament phosphorylation. Biochem Cell Biol, 73, 57592.

PARK, A. J., HAVEKES, R., CHOI, J. H. K., LUCZAK, V., NIE, T., HUANG, T. \& ABEL, T. 2014. A presynaptic role for PKA in synaptic tagging and memory. Neurobiology of learning and memory, 114, 101-112.

PARK, J. H., JUNG, M. S., KIM, Y. S., SONG, W. J. \& CHUNG, S. H. 2012. Phosphorylation of Munc18-1 by Dyrk1A regulates its interaction with Syntaxin 1 and X11alpha. $J$ Neurochem, 122, 1081-91.

PAULO, J. A. 2013. Practical and Efficient Searching in Proteomics: A Cross Engine Comparison. WebmedCentral, 4, WMCPLS0052.

PENG, A., ROTMAN, Z., DENG, P. Y. \& KLYACHKO, V. A. 2012. Differential motion dynamics of synaptic vesicles undergoing spontaneous and activity-evoked endocytosis. Neuron, 73, 1108-15.

PERRINO, B. A., NG, L. Y. \& SODERLING, T. R. 1995. Calcium Regulation of Calcineurin Phosphatase Activity by Its B Subunit and Calmodulin: ROLE OF THE AUTOINHIBITORY DOMAIN. Journal of Biological Chemistry, 270, 340-346.

POPOLI, M. 1993. Synaptotagmin is endogenously phosphorylated by $\mathrm{Ca}+$ /calmodulin protein kinase II in synaptic vesicles. FEBS Lett, 317, 85-8. 
PREKERIS, R. \& TERRIAN, D. M. 1997. Brain Myosin V Is a Synaptic Vesicle-associated Motor Protein: Evidence for a $\mathrm{Ca}(2+)$-dependent Interaction with the SynaptobrevinSynaptophysin Complex. The Journal of Cell Biology, 137, 1589-1601.

QIAN, W., SHI, J., YIN, X., IQBAL, K., GRUNDKE-IQBAL, I., GONG, C. X. \& LIU, F. 2010. PP2A regulates tau phosphorylation directly and also indirectly via activating GSK-3beta. J Alzheimers Dis, 19, 1221-9.

QUAN L, L. M. 2013. CID, ETD and HCD Fragmentation to Study Protein Post-Translational Modifications. Mod Chem appl 1, 102.

RELLOS, P., PIKE, A. C., NIESEN, F. H., SALAH, E., LEE, W. H., VON DELFT, F. \& KNAPP, S. 2010. Structure of the CaMKIIdelta/calmodulin complex reveals the molecular mechanism of CaMKII kinase activation. PLoS Biol, 8, e1000426.

REN, J., WEN, L., GAO, X., JIN, C., XUE, Y. \& YAO, X. 2009. DOG 1.0: illustrator of protein domain structures. Cell Res, 19, 271-273.

RENARD, H. F., SIMUNOVIC, M., LEMIERE, J., BOUCROT, E., GARCIA-CASTILLO, M. D., ARUMUGAM, S., CHAMBON, V., LAMAZE, C., WUNDER, C., KENWORTHY, A. K., SCHMIDT, A. A., MCMAHON, H. T., SYKES, C., BASSEREAU, P. \& JOHANNES, L. 2015. Endophilin-A2 functions in membrane scission in clathrin-independent endocytosis. Nature, 517, 493-6.

RICKMAN, C. \& DUNCAN, R. R. 2010. Munc18/Syntaxin interaction kinetics control secretory vesicle dynamics. J Biol Chem, 285, 3965-72.

RIZO, J., CHEN, X. \& ARAC, D. 2006. Unraveling the mechanisms of synaptotagmin and SNARE function in neurotransmitter release. Trends Cell Biol, 16, 339-50.

RIZZOLI, S. O. \& BETZ, W. J. 2005. Synaptic vesicle pools. Nat Rev Neurosci, 6, 57-69.

RIZZOLI, S. O. \& JAHN, R. 2007. Kiss-and-run, collapse and 'readily retrievable' vesicles. Traffic, 8, 1137-44.

ROACH, P. J. 1991. Multisite and hierarchal protein phosphorylation. J Biol Chem, 266, 1413942.

RODINA, V. I., SALATA, L. K., ROZHANETS, V. V., POZDNYAKOV, O. M. \& GLEBOV, R. N. 1980. Ultrastructural and functional changes in rat brain synaptosomes during electrical stimulation in vitro. Bulletin of Experimental Biology and Medicine, 89, 108-111.

RODRIGUEZ, A., ROY, J., MARTINEZ-MARTINEZ, S., LOPEZ-MADERUELO, M. D., NINOMORENO, P., ORTI, L., PANTOJA-UCEDA, D., PINEDA-LUCENA, A., CYERT, M. S. \& REDONDO, J. M. 2009. A conserved docking surface on calcineurin mediates interaction with substrates and immunosuppressants. Mol Cell, 33, 616-26.

ROEPSTORFF, P. \& FOHLMAN, J. 1984. Proposal for a common nomenclature for sequence ions in mass spectra of peptides. Biomed Mass Spectrom, 11, 601.

ROSKOSKI JR, R. 2012. ERK1/2 MAP kinases: Structure, function, and regulation. Pharmacological Research, 66, 105-143.

RUIZ, R., CANO, R., CASANAS, J. J., GAFFIELD, M. A., BETZ, W. J. \& TABARES, L. 2011. Active zones and the readily releasable pool of synaptic vesicles at the neuromuscular junction of the mouse. J Neurosci, 31, 2000-8.

RUSH, J., MORITZ, A., LEE, K. A., GUO, A., GOSS, V. L., SPEK, E. J., ZHANG, H., ZHA, X.-M., POLAKIEWICZ, R. D. \& COMB, M. J. 2005. Immunoaffinity profiling of tyrosine phosphorylation in cancer cells. Nat Biotech, 23, 94-101.

RYAN, T. A., LI, L., CHIN, L. S., GREENGARD, P. \& SMITH, S. J. 1996. Synaptic vesicle recycling in synapsin I knock-out mice. J Cell Biol, 134, 1219-27. 
RYLATT, D. B., AITKEN, A., BILHAM, T., CONDON, G. D., EMBI, N. \& COHEN, P. 1980. Glycogen synthase from rabbit skeletal muscle. Amino acid sequence at the sites phosphorylated by glycogen synthase kinase-3, and extension of the $\mathrm{N}$-terminal sequence containing the site phosphorylated by phosphorylase kinase. European Journal of Biochemistry, 107, 529-537.

RYU, J. K., JAHN, R. \& YOON, T. Y. 2016. Review: Progresses in understanding Nethylmaleimide sensitive factor (NSF) mediated disassembly of SNARE complexes. Biopolymers, 105, 518-31.

SACCO, F., PERFETTO, L., CASTAGNOLI, L. \& CESARENI, G. 2012. The human phosphatase interactome: An intricate family portrait. Febs Letters, 586, 2732-2739.

SAHEKI, Y. \& DE CAMILLI, P. 2012. Synaptic vesicle endocytosis. Cold Spring Harb Perspect Biol, 4, a005645.

SALAZAR, C. \& HÖFER, T. 2009. Multisite protein phosphorylation - from molecular mechanisms to kinetic models. FEBS Journal, 276, 3177-3198.

SALZANO, M., RUSCIANO, M. R., RUSSO, E., BIFULCO, M., POSTIGLIONE, L. \& VITALE, M. 2012. Calcium/calmodulin-dependent protein kinase II (CaMKII) phosphorylates Raf-1 at serine 338 and mediates Ras-stimulated Raf-1 activation. Cell Cycle, 11, 2100-6.

SAMUELS, B. A. \& TSAI, L. H. 2003. Cdk5 is a dynamo at the synapse. Nat Cell Biol, 5, 689-90.

SANDERSON, J. L. \& DELL'ACQUA, M. L. 2011. AKAP signaling complexes in regulation of excitatory synaptic plasticity. Neuroscientist, 17, 321-36.

SASAKI, Y., CHENG, C., UCHIDA, Y., NAKAJIMA, O., OHSHIMA, T., YAGI, T., TANIGUCHI, M., NAKAYAMA, T., KISHIDA, R., KUDO, Y., OHNO, S., NAKAMURA, F. \& GOSHIMA, Y. 2002. Fyn and Cdk5 mediate semaphorin-3A signaling, which is involved in regulation of dendrite orientation in cerebral cortex. Neuron, 35, 907-20.

SAURIN, A. T., DURGAN, J., CAMERON, A. J., FAISAL, A., MARBER, M. S. \& PARKER, P. J. 2008. The regulated assembly of a PKCepsilon complex controls the completion of cytokinesis. Nat Cell Biol, 10, 891-901.

SCHIEBLER, W., JAHN, R., DOUCET, J. P., ROTHLEIN, J. \& GREENGARD, P. 1986. Characterization of synapsin I binding to small synaptic vesicles. J Biol Chem, 261, 8383-90.

SCHIKORSKI, T. \& STEVENS, C. F. 2001. Morphological correlates of functionally defined synaptic vesicle populations. Nat Neurosci, 4, 391-395.

SCHMIDT, A. \& HUTTNER, W. B. 1998. Biogenesis of synaptic-like microvesicles in perforated PC12 cells. Methods, 16, 160-9.

SCHMIDT, A., KARAS, M. \& DÜLCKS, T. 2003. Effect of different solution flow rates on analyte ion signals in nano-ESI MS, or: when does ESI turn into nano-ESI? Journal of the American Society for Mass Spectrometry, 14, 492-500.

SCHMITZ, S. K., KING, C., KORTLEVEN, C., HUSON, V., KROON, T., KEVENAAR, J. T., SCHUT, D., SAARLOOS, I., HOETJES, J. P., DE WIT, H., STIEDL, O., SPIJKER, S., LI, K. W., MANSVELDER, H. D., SMIT, A. B., CORNELISSE, L. N., VERHAGE, M. \& TOONEN, R. F. 2016. Presynaptic inhibition upon CB1 or mGlu2/3 receptor activation requires ERK/MAPK phosphorylation of Munc18-1. Embo j, 35, 1236-50.

SCHOCH, S., CASTILLO, P. E., JO, T., MUKHERJEE, K., GEPPERT, M., WANG, Y., SCHMITZ, F., MALENKA, R. C. \& SUDHOF, T. C. 2002. RIM1alpha forms a protein scaffold for regulating neurotransmitter release at the active zone. Nature, 415, 321-6.

SCHRODER, M. S., STELLMACHER, A., ROMORINI, S., MARINI, C., MONTENEGRO-VENEGAS, C., ALTROCK, W. D., GUNDELFINGER, E. D. \& FEJTOVA, A. 2013. Regulation of presynaptic 
anchoring of the scaffold protein Bassoon by phosphorylation-dependent interaction with 14-3-3 adaptor proteins. PLoS One, 8, e58814.

SCHWARTZ, D. \& GYGI, S. P. 2005. An iterative statistical approach to the identification of protein phosphorylation motifs from large-scale data sets. Nat Biotechnol, 23, 1391-8. SCIGELOVA, M. \& MAKAROV, A. 2006. Orbitrap mass analyzer--overview and applications in proteomics. Proteomics, 6 Suppl 2, 16-21.

SCOTT, R. 2007. Use-dependent control of presynaptic calcium signalling at central synapses. J Anat, 210, 642-50.

SEABROOKE, S. \& STEWART, B. A. 2011. Synaptic transmission and plasticity are modulated by nonmuscle myosin II at the neuromuscular junction of Drosophila. J Neurophysiol, 105, 1966-76.

SHAH, K. \& LAHIRI, D. K. 2014. Cdk5 activity in the brain - multiple paths of regulation. J Cell Sci, 127, 2391-400.

SHARMA, K., D'SOUZA, R. C., TYANOVA, S., SCHAAB, C., WISNIEWSKI, J. R., COX, J. \& MANN, M. 2014. Ultradeep human phosphoproteome reveals a distinct regulatory nature of Tyr and Ser/Thr-based signaling. Cell Rep, 8, 1583-94.

SHATA, A., SAISU, H., ODANI, S. \& ABE, T. 2007. Phosphorylated synaphin/complexin found in the brain exhibits enhanced SNARE complex binding. Biochemical and Biophysical Research Communications, 354, 808-813.

SHEVCHENKO, A., LOBODA, A., SHEVCHENKO, A., ENS, W. \& STANDING, K. G. 2000. MALDI quadrupole time-of-flight mass spectrometry: a powerful tool for proteomic research. Anal Chem, 72, 2132-41.

SHEVCHENKO, A., WILM, M., VORM, O., JENSEN, O. N., PODTELEJNIKOV, A. V., NEUBAUER, G., SHEVCHENKO, A., MORTENSEN, P. \& MANN, M. 1996. A strategy for identifying gelseparated proteins in sequence databases by MS alone. Biochem Soc Trans, 24, 893-6.

SIHRA, T. S., NAIRN, A. C., KLOPPENBURG, P., LIN, Z. X. \& POUZAT, C. 1995. A Role for Calcineurin (Protein Phosphatase-2B) in the Regulation of Glutamate Release. Biochemical and Biophysical Research Communications, 212, 609-616.

SIMSEK-DURAN, F. \& LONART, G. 2008. The role of RIM1alpha in BDNF-enhanced glutamate release. Neuropharmacology, 55, 27-34.

SINDREU, C. B., SCHEINER, Z. S. \& STORM, D. R. 2007. Ca2+-Stimulated Adenylyl Cyclases Regulate ERK-Dependent Activation of MSK1 during Fear Conditioning. Neuron, 53, 79-89.

SLEPNEV, V. I. \& DE CAMILLI, P. 2000. Accessory factors in clathrin-dependent synaptic vesicle endocytosis. Nat Rev Neurosci, 1, 161-172.

SMILLIE, K. J. \& COUSIN, M. A. 2011. The Role of GSK3 in Presynaptic Function. Int J Alzheimers Dis, 2011, 263673.

SOLYAKOV, L., CAIN, K., TRACEY, B. M., JUKES, R., RILEY, A. M., POTTER, B. V. L. \& TOBIN, A. B. 2004. Regulation of Casein Kinase-2 (CK2) Activity by Inositol Phosphates. Journal of Biological Chemistry, 279, 43403-43410.

SOMLYO, A. P. \& SOMLYO, A. V. 2003. Ca2+ sensitivity of smooth muscle and nonmuscle myosin II: modulated by G proteins, kinases, and myosin phosphatase. Physiol Rev, 83, 1325-58.

SONG, B., LAI, B., ZHENG, Z., ZHANG, Y., LUO, J., WANG, C., CHEN, Y., WOODGETT, J. R. \& LI, M. 2010. Inhibitory phosphorylation of GSK-3 by CaMKII couples depolarization to neuronal survival. J Biol Chem, 285, 41122-34. 
SOYKAN, T., MARITZEN, T. \& HAUCKE, V. 2016. Modes and mechanisms of synaptic vesicle recycling. Current Opinion in Neurobiology, 39, 17-23.

STEEN, H., KÜSTER, B., FERNANDEZ, M., PANDEY, A. \& MANN, M. 2001. Detection of Tyrosine Phosphorylated Peptides by Precursor Ion Scanning Quadrupole TOF Mass Spectrometry in Positive Ion Mode. Analytical Chemistry, 73, 1440-1448.

STEEN, H. \& MANN, M. 2004. The abc's (and xyz's) of peptide sequencing. Nat Rev Mol Cell Biol, 5, 699-711.

STEINBERG, S. F. 2008. Structural Basis of Protein Kinase C Isoform Function. Physiological Reviews, 88, 1341-1378.

STEVENS, C. F. \& SULLIVAN, J. M. 1998. Regulation of the readily releasable vesicle pool by protein kinase C. Neuron, 21, 885-93.

STRYKER, E. \& JOHNSON, K. G. 2007. LAR, liprin $\alpha$ and the regulation of active zone morphogenesis. Journal of Cell Science, 120, 3723-3728.

SU, S. C., SEO, J., PAN, J. Q., SAMUELS, B. A., RUDENKO, A., ERICSSON, M., NEVE, R. L., YUE, D. T. \& TSAI, L. H. 2012. Regulation of N-type voltage-gated calcium channels and presynaptic function by cyclin-dependent kinase 5 . Neuron, 75, 675-87.

SUDHOF, T. C. 2013. Neurotransmitter release: the last millisecond in the life of a synaptic vesicle. Neuron, 80, 675-90.

SUN, L., BITTNER, M. A. \& HOLZ, R. W. 2003. Rim, a component of the presynaptic active zone and modulator of exocytosis, binds 14-3-3 through its N terminus. J Biol Chem, 278, 38301-9.

SUN, T., WU, X. S., XU, J., MCNEIL, B. D., PANG, Z. P., YANG, W., BAI, L., QADRI, S., MOLKENTIN, J. D., YUE, D. T. \& WU, L. G. 2010. The role of calcium/calmodulin-activated calcineurin in rapid and slow endocytosis at central synapses. J Neurosci, 30, 11838-47.

SUNDBORGER, A. C. \& HINSHAW, J. E. 2014. Regulating dynamin dynamics during endocytosis. F1000Prime Rep, 6, 85.

TAGAWA, K., HOMMA, H., SAITO, A., FUJITA, K., CHEN, X., IMOTO, S., OKA, T., ITO, H., MOTOKI, K., YOSHIDA, C., HATSUTA, H., MURAYAMA, S., IWATSUBO, T., MIYANO, S. \& OKAZAWA, H. 2015. Comprehensive phosphoproteome analysis unravels the core signaling network that initiates the earliest synapse pathology in preclinical Alzheimer's disease brain. Hum Mol Genet, 24, 540-58.

TAKAMORI, S., HOLT, M., STENIUS, K., LEMKE, E. A., GRONBORG, M., RIEDEL, D., URLAUB, H., SCHENCK, S., BRUGGER, B., RINGLER, P., MULLER, S. A., RAMMNER, B., GRATER, F., HUB, J. S., DE GROOT, B. L., MIESKES, G., MORIYAMA, Y., KLINGAUF, J., GRUBMULLER, H., HEUSER, J., WIELAND, F. \& JAHN, R. 2006. Molecular anatomy of a trafficking organelle. Cell, 127, 831-46.

TANAKA, K., WAKI, H., IDO, Y., AKITA, S., YOSHIDA, Y., YOSHIDA, T. AND MATSUO, T. 1988. Protein and polymer analyses up to m/z 100000 by laser ionization time-of-flight mass spectrometry. Rapid Commun. Mass Spectrom, 2, 151-153.

TANIGUCHI, M., TAOKA, M., ITAKURA, M., ASADA, A., SAITO, T., KINOSHITA, M., TAKAHASHI, M., ISOBE, T. \& HISANAGA, S. 2007. Phosphorylation of adult type Sept5 (CDCrel-1) by cyclin-dependent kinase 5 inhibits interaction with syntaxin-1.J Biol Chem, 282, 786976.

TIAN, J. H., DAS, S. \& SHENG, Z. H. 2003. Ca2+-dependent phosphorylation of syntaxin-1A by the death-associated protein (DAP) kinase regulates its interaction with Munc18. J Biol Chem, 278, 26265-74. 
TIBBS, G. R., BARRIE, A. P., VAN MIEGHEM, F. J., MCMAHON, H. T. \& NICHOLLS, D. G. 1989. Repetitive action potentials in isolated nerve terminals in the presence of 4aminopyridine: effects on cytosolic free Ca2+ and glutamate release. J Neurochem, 53, 1693-9.

TOMIZAWA, K., SUNADA, S., LU, Y.-F., ODA, Y., KINUTA, M., OHSHIMA, T., SAITO, T., WEI, F.-Y., MATSUSHITA, M., LI, S.-T., TSUTSUI, K., HISANAGA, S.-I., MIKOSHIBA, K., TAKEI, K. \& MATSUI, H. 2003a. Cophosphorylation of amphiphysin I and dynamin I by Cdk5 regulates clathrin-mediated endocytosis of synaptic vesicles. The Journal of Cell Biology, 163, 813-824.

TOMIZAWA, K., SUNADA, S., LU, Y. F., ODA, Y., KINUTA, M., OHSHIMA, T., SAITO, T., WEI, F. Y., MATSUSHITA, M., LI, S. T., TSUTSUI, K., HISANAGA, S., MIKOSHIBA, K., TAKEI, K. \& MATSUI, H. 2003b. Cophosphorylation of amphiphysin I and dynamin I by Cdk5 regulates clathrin-mediated endocytosis of synaptic vesicles. J Cell Biol, 163, 813-24.

TRINIDAD, J. C., BARKAN, D. T., GULLEDGE, B. F., THALHAMMER, A., SALI, A., SCHOEPFER, R. \& BURLINGAME, A. L. 2012. Global identification and characterization of both 0GlcNAcylation and phosphorylation at the murine synapse. Mol Cell Proteomics, 11, 215-29.

TRINIDAD, J. C., SPECHT, C. G., THALHAMMER, A., SCHOEPFER, R. \& BURLINGAME, A. L. 2006. Comprehensive identification of phosphorylation sites in postsynaptic density preparations. Mol Cell Proteomics, 5, 914-22.

TRINIDAD, J. C., THALHAMMER, A., SPECHT, C. G., LYNN, A. J., BAKER, P. R., SCHOEPFER, R. \& BURLINGAME, A. L. 2008. Quantitative analysis of synaptic phosphorylation and protein expression. Mol Cell Proteomics, 7, 684-96.

TSAI, L.-H., DELALLE, I., CAVINESS, V. S., CHAE, T. \& HARLOW, E. 1994. p35 is a neural-specific regulatory subunit of cyclin-dependent kinase 5. Nature, 371, 419-423.

VAROQUEAUX, F., SIGLER, A., RHEE, J.-S., BROSE, N., ENK, C., REIM, K. \& ROSENMUND, C. 2002. Total arrest of spontaneous and evoked synaptic transmission but normal synaptogenesis in the absence of Munc13-mediated vesicle priming. Proceedings of the National Academy of Sciences of the United States of America, 99, 9037-9042.

VENERANDO, A., RUZZENE, M. \& PINNA, L. A. 2014. Casein kinase: the triple meaning of a misnomer. Biochem J, 460, 141-56.

VERHAGE, M., BESSELSEN, E., LOPES DA SILVA, F. H. \& GHIJSEN, W. E. 1988. Evaluation of the $\mathrm{Ca} 2+$ concentration in purified nerve terminals: relationship between $\mathrm{Ca} 2+$ homeostasis and synaptosomal preparation. J Neurochem, 51, 1667-74.

VERHAGE, M., MAIA, A. S., PLOMP, J. J., BRUSSAARD, A. B., HEEROMA, J. H., VERMEER, H., TOONEN, R. F., HAMMER, R. E., VAN DEN BERG, T. K., MISSLER, M., GEUZE, H. J. \& SUDHOF, T. C. 2000. Synaptic assembly of the brain in the absence of neurotransmitter secretion. Science, 287, 864-9.

VERSTEGEN, A. M., TAGLIATTI, E., LIGNANI, G., MARTE, A., STOLERO, T., ATIAS, M., CORRADI, A., VAlTORTA, F., GITLER, D., ONOFRI, F., FASSIO, A. \& BENFENATI, F. 2014. Phosphorylation of synapsin I by cyclin-dependent kinase- 5 sets the ratio between the resting and recycling pools of synaptic vesicles at hippocampal synapses. $J$ Neurosci, 34, 7266-80.

VILLEN, J. \& GYGI, S. P. 2008. The SCX/IMAC enrichment approach for global phosphorylation analysis by mass spectrometry. Nat Protoc, 3, 1630-8.

VILLÉN, J. \& GYGI, S. P. 2008. The SCX/IMAC enrichment approach for global phosphorylation analysis by mass spectrometry. Nature protocols, 3, 1630-1638. 
WAITES, C. L., LEAL-ORTIZ, S. A., ANDLAUER, T. F., SIGRIST, S. J. \& GARNER, C. C. 2011. Piccolo regulates the dynamic assembly of presynaptic F-actin. J Neurosci, 31, 14250-63.

WALAAS, S. I., ASWAD, D. W. \& GREENGARD, P. 1983. A dopamine- and cyclic AMP-regulated phosphoprotein enriched in dopamine-innervated brain regions. Nature, 301, 69-71.

WANG, J. Z., XIA, Y. Y., GRUNDKE-IQBAL, I. \& IQBAL, K. 2013. Abnormal hyperphosphorylation of tau: sites, regulation, and molecular mechanism of neurofibrillary degeneration. $J$ Alzheimers Dis, 33 Suppl 1, S123-39.

WANG, Y., SUGITA, S. \& SUDHOF, T. C. 2000. The RIM/NIM family of neuronal C2 domain proteins. Interactions with Rab3 and a new class of Src homology 3 domain proteins. J Biol Chem, 275, 20033-44.

WATANABE, S., ROST, B. R., CAMACHO-PEREZ, M., DAVIS, M. W., SOHL-KIELCZYNSKI, B., ROSENMUND, C. \& JORGENSEN, E. M. 2013. Ultrafast endocytosis at mouse hippocampal synapses. Nature, 504, 242-247.

WATANABE, S., TRIMBUCH, T., CAMACHO-PEREZ, M., ROST, B. R., BROKOWSKI, B., SOHLKIELCZYNSKI, B., FELIES, A., DAVIS, M. W., ROSENMUND, C. \& JORGENSEN, E. M. 2014. Clathrin regenerates synaptic vesicles from endosomes. Nature, 515, 228-233.

WATERS, J. \& SMITH, S. J. 2002. Vesicle pool partitioning influences presynaptic diversity and weighting in rat hippocampal synapses. The Journal of Physiology, 541, 811-823.

WAYMAN, G. A., LEE, Y.-S., TOKUMITSU, H., SILVA, A. \& SODERLING, T. R. 2008. CalmodulinKinases: Modulators of Neuronal Development and Plasticity. Neuron, 59, 914-931.

WEI, F. Y., TOMIZAWA, K., OHSHIMA, T., ASADA, A., SAITO, T., NGUYEN, C., BIBB, J. A., ISHIGURO, K., KULKARNI, A. B., PANT, H. C., MIKOSHIBA, K., MATSUI, H. \& HISANAGA, S. 2005. Control of cyclin-dependent kinase 5 (Cdk5) activity by glutamatergic regulation of p35 stability. J Neurochem, 93, 502-12.

WHITTAKER, V. P. 1993. Thirty years of synaptosome research. J Neurocytol, 22, 735-42.

WILDE, A. \& BRODSKY, F. M. 1996. In vivo phosphorylation of adaptors regulates their interaction with clathrin. J Cell Biol, 135, 635-45.

WINDER, D. G. \& SWEATT, J. D. 2001. Roles of serine/threonine phosphatases in hippocampel synaptic plasticity. Nat Rev Neurosci, 2, 461-474.

WISNIEWSKI, J. R., ZOUGMAN, A., NAGARAJ, N. \& MANN, M. 2009. Universal sample preparation method for proteome analysis. Nat Meth, 6, 359-362.

WORTH, D. C., DALY, C. N., GERALDO, S., OOZEER, F. \& GORDON-WEEKS, P. R. 2013. Drebrin contains a cryptic F-actin-bundling activity regulated by Cdk5 phosphorylation. J Cell Biol, 202, 793-806.

XUE, M., CRAIG, T. K., XU, J., CHAO, H.-T., RIZO, J. \& ROSENMUND, C. 2010. Binding of the complexin $\mathrm{N}$ terminus to the SNARE complex potentiates synaptic-vesicle fusogenicity. Nat Struct Mol Biol, 17, 568-575.

YAFFE, M. B. \& ELIA, A. E. 2001. Phosphoserine/threonine-binding domains. Curr Opin Cell Biol, 13, 131-8.

YAMAUCHI, T. 2005. Neuronal Ca2+/calmodulin-dependent protein kinase II--discovery, progress in a quarter of a century, and perspective: implication for learning and memory. Biol Pharm Bull, 28, 1342-54.

YOKOYAMA, C. T., SHENG, Z. H. \& CATTERALL, W. A. 1997. Phosphorylation of the synaptic protein interaction site on $\mathrm{N}$-type calcium channels inhibits interactions with SNARE proteins. Journal of Neuroscience, 17, 6929-6938. 
YUE, X., SCHUNTER, A. \& HUMMON, A. B. 2015. Comparing multistep immobilized metal affinity chromatography and multistep TiO2 methods for phosphopeptide enrichment. Anal Chem, 87, 8837-44.

ZARGAR, S. M., KURATA, R., RAKWAL, R. \& FUKAO, Y. 2015. Peptide separation methodologies for in-depth proteomics. Methods Mol Biol, 1242, 195-209.

ZHANG, L., MARCU, M. G., NAU-STAUDT, K. \& TRIFARÓ, J. M. 1996. Recombinant Scinderin Enhances Exocytosis, an Effect Blocked by Two Scinderin-Derived Actin-Binding Peptides and PIP2. Neuron, 17, 287-296.

ZHANG, M., PATRIARCHI, T., STEIN, I. S., QIAN, H., MATT, L., NGUYEN, M., XIANG, Y. K. \& HELL, J. W. 2013a. Adenylyl Cyclase Anchoring by a Kinase Anchor Protein AKAP5 (AKAP79/150) Is Important for Postsynaptic $\beta$-Adrenergic Signaling. Journal of Biological Chemistry, 288, 17918-17931.

ZHANG, M. Y., PACE, N., KERNS, E. H., KLEINTOP, T., KAGAN, N. \& SAKUMA, T. 2005. Hybrid triple quadrupole-linear ion trap mass spectrometry in fragmentation mechanism studies: application to structure elucidation of buspirone and one of its metabolites. $J$ Mass Spectrom, 40, 1017-29.

ZHANG, Y., FONSLOW, B. R., SHAN, B., BAEK, M. C. \& YATES, J. R., 3RD 2013b. Protein analysis by shotgun/bottom-up proteomics. Chem Rev, 113, 2343-94.

ZHU, L. Q., LIU, D., HU, J., CHENG, J., WANG, S. H., WANG, Q., WANG, F., CHEN, J. G. \& WANG, J. Z. 2010. GSK-3 beta inhibits presynaptic vesicle exocytosis by phosphorylating P/Qtype calcium channel and interrupting SNARE complex formation. J Neurosci, 30, 3624-33.

ZUBAREV, R. A. \& MAKAROV, A. 2013. Orbitrap Mass Spectrometry. Analytical Chemistry, 85, 5288-5296.

ZUKERBERG, L. R., PATRICK, G. N., NIKOLIC, M., HUMBERT, S., WU, C. L., LANIER, L. M., GERTLER, F. B., VIDAL, M., VAN ETTEN, R. A. \& TSAI, L. H. 2000. Cables links Cdk5 and c-Abl and facilitates Cdk5 tyrosine phosphorylation, kinase upregulation, and neurite outgrowth. Neuron, 26, 633-46. 


\section{Appendices}

\subsection{List of abbreviations}

\begin{tabular}{ll}
$\mu \mathrm{g}$ & Microgram \\
$\mu \mathrm{M}$ & Micromolar \\
ACN & Acetonitiril \\
ADBE & Activity-Dependent Bulk Endocytosis \\
ADP & Adenosine diphosphate \\
AP & Action potential \\
ATP & Adenosine triphosphate \\
CAA & 2-Chloroacetamide \\
CaM & Calmodulin \\
CaMKII & Ca $2+$ /calmodulindependent kinase II \\
CdK5 & Cyclin-dependent Kinase 5 \\
CID & Collision-Induced Dissociation \\
CK2 & Casein Kinase 2 \\
CME & Clathrin-Mediated Endocytosis \\
CycloA & Cyclosporine A \\
Da & Dalton \\
DARPP-32 & Dopamine- and cAMP-Regulated Phospho Protein 32 \\
DDA & Data-Dependent Acquisition \\
DHB & 2,5-dihydroxybenzoic acid \\
DTT & Dithiothreitol \\
ERK 1/2 & Extracellular-signal Regulated Kinase 1/2 \\
ESI & Electrospray ionization \\
ETD & Electron Transfer Dissociation \\
FA & Formid acid \\
FDR & False Discovery Rate \\
g (RCF) & Relative centrifugal force (RCF) \\
GluDH & Glutamate dehydrogenase \\
GSK-3 & Glycogen Synthase Kinase 3 \\
h & Hour \\
HCD & High-energy Collisional Dissociation \\
HPLC & High-Performance Liquid Chromatography \\
Hz & Hertz \\
ID & Inner diametere \\
IMAC & Immobilized Metal Affinity Chromatography \\
LC & Liquid chromatography \\
M & Molar \\
m/z & Mass to charge ratio \\
MALDI & Matrix-Assisted Laser Desorption Ionization \\
\hline
\end{tabular}




\begin{tabular}{ll} 
min & Minute \\
ml & Mililitre \\
mM & Milimolar \\
MS & Mass spectrometry \\
MS/MS & Tandem Mass spectremtry \\
MS1 & Peptide precursor spectrum \\
MS2 & Product ion spectrum \\
msec/ms & Millisecond \\
nL & Nanoliter \\
nM & Nanomolar \\
PDK-1 & Phosphoinositide-dependent kinase-1 \\
PKA & Protein Kinase A \\
PKC & Protein Kinase C \\
PP & Protein phosphatases \\
pS & Phosphorylated Serine \\
pT & Phosphorylated Threonine \\
PTM & Post translational modification \\
pY & Phosphorylated Tyrosine \\
RP & Reverse Phase \\
rpm & Round per minute \\
RRP & Readily releasable pool \\
RT & Room tempreture \\
S/Ser & Serine \\
SCX & Strong Cation Exchange \\
SDS-PAGE & Sodium dodecyl sulfate polyacrylamide gel electrophoresis \\
sec & Second \\
SILAC & Stable Isotope Labeling with Amino acids \\
SV & Synaptic vesicle \\
T/Thr & Threonine \\
TEAB & Triethylammonium bicarbonate buffer \\
TFA & Trifluoroacetic acid \\
TiO 2 & Titanium dioxide \\
U & Unit \\
V & Volt \\
Vol & Volume \\
WB & Western Blot \\
Y/Tyr & Tyrosine \\
& \\
\hline Th & \\
\hline
\end{tabular}




\subsection{Supplementary Tables}

In this section all the regulated phosphosites in different experiments are listed. The list of all quantified phosphosites (regulated or not regulated) are stored on CD that can be found attached at the back of the hard copy of this thesis.

\begin{tabular}{|l|l|l|}
\hline Experiment & Regulates phosphosites & $\begin{array}{l}\text { All the quantified } \\
\text { phosphosites on the CD }\end{array}$ \\
\hline Comparison $\mathrm{K}^{+}$, EGTA vs K+ $\mathrm{Ca}^{2+}$ & Table 6-1 & Table S1 \\
\hline Comparison EGTA vs $\mathrm{K}^{+}, \mathrm{Ca}^{2+}$ & Table 6-2 & Table S2 \\
\hline Comparison $\mathrm{K}^{+}$, EGTA vs EGTA & Table 6-3 & Table S3 \\
\hline Inhibition of Calcineurin & Table 6-4, Table 6-5 & Table S4 \\
\hline Inhibition of CaMKII & Table 6-6 & Table S5 \\
\hline
\end{tabular}

Table 6-1: List of regulated phosphosites in comparison $\mathrm{K}^{+}, \mathrm{EGTA}$ vs $\mathrm{K}^{+}, \mathrm{Ca}^{2+}$

\begin{tabular}{|l|l|l|l|}
\hline Regulation & Protein names & Gene names & $\begin{array}{l}\text { Aminoacid/ } \\
\text { position }\end{array}$ \\
\hline Downregulated & AP2-associated protein kinase 1 & Aak1 & S639 \\
\hline Downregulated & AP2-associated protein kinase 1 & Aak1 & S672 \\
\hline Downregulated & AP2-associated protein kinase 1 & Aak1 & S670 \\
\hline Upregulated & ATP citrate lyase & Acly & S455 \\
\hline Upregulated & Calcium/calmodulin-dependent protein kinase II & Camk2b & T401 \\
\hline Upregulated & $\begin{array}{l}\text { Calcium/calmodulin-dependent protein kinase type II subunit } \\
\text { gamma }\end{array}$ & Camk2g & S299 \\
\hline Upregulated & $\begin{array}{l}\text { Calcium/calmodulin-dependent protein kinase type II subunit } \\
\text { gamma }\end{array}$ & Camk2g & S300 \\
\hline Downregulated & Calcium-dependent secretion activator 1 & Cadps & S89 \\
\hline Downregulated & Calcium-dependent secretion activator 1 & Cadps & S88 \\
\hline Upregulated & Catenin delta-2 & Ctnnd2 & S459 \\
\hline Downregulated & Clathrin coat assembly protein AP180 & Snap91 & S600 \\
\hline Downregulated & Clathrin coat assembly protein AP180 & Snap91 & S627 \\
\hline Downregulated & Clathrin coat assembly protein AP180 & Snap91 & S621 \\
\hline Upregulated & Complexin-1 & Cplx1 & S93 \\
\hline Upregulated & Dihydropyrimidinase-related protein 1 & Crmp1 1 & S542 \\
\hline Upregulated & Dihydropyrimidinase-related protein 2 & Dpysl2 & S542 \\
\hline Upregulated & Dihydropyrimidinase-related protein 2 & Dpys12 & S540 \\
\hline Upregulated & Dihydropyrimidinase-related protein 2 & Dpysl5 & S445 \\
\hline Upregulated & Disks large-associated protein 2 & Dlgap2 & S977 \\
\hline Downregulated & DnaJ (Hsp40) homolog, subfamily C, member 6 & Dnajc6 & S652 \\
\hline Downregulated & DnaJ (Hsp40) homolog, subfamily C, member 6 & Dnajc6 & T658 \\
\hline Downregulated & DnaJ (Hsp40) homolog, subfamily C, member 6 & Dnajc6 & T655 \\
\hline Upregulated & Drebrin & Dbn1 & S241 \\
\hline
\end{tabular}




\begin{tabular}{|c|c|c|c|}
\hline Upregulated & Drebrin-like protein & Dbnl Sh3p7 & S277 \\
\hline Upregulated & Dynamin-1 & Dnm1 Dnm & S778 \\
\hline Downregulated & Dynamin-1 & Dnm1 Dnm & S774 \\
\hline Downregulated & Dynamin-1 & Dnm1 Dnm & T776 \\
\hline Downregulated & Dynamin-1 & Dnm1 Dnm & S853 \\
\hline Downregulated & Dynamin-1 & Dnm1 Dnm & S857 \\
\hline Downregulated & Dynamin-1 & Dnm1 Dnm & S817 \\
\hline Downregulated & Dynamin-1 & Dnm1 Dnm & S822 \\
\hline Downregulated & ELKS/Rab6-interacting/CAST family member 1 & Erc1 & S21 \\
\hline Downregulated & Endophilin-A2 & Sh3gl1 Sh3p8 & S288 \\
\hline Upregulated & Epsin-1 & Epn1 & S450 \\
\hline Downregulated & ERC protein 2 & Erc2 & S16 \\
\hline Downregulated & ERC protein 2 & Erc2 & S18 \\
\hline Downregulated & Erythrocyte protein band 4.1-like 3, isoform CRA_b & Epb4113 & S578 \\
\hline Downregulated & FXYD domain-containing ion transport regulator 7 & Fxyd7 & S60 \\
\hline Downregulated & FXYD domain-containing ion transport regulator 7 & Fxyd7 & S56 \\
\hline Downregulated & FXYD domain-containing ion transport regulator 7 & Fxyd7 & S58 \\
\hline Upregulated & Glutamate decarboxylase 1 & Gad1 Gad67 & T53 \\
\hline Upregulated & Glutamate decarboxylase 1 & Gad1 Gad67 & S55 \\
\hline Upregulated & Glycine--tRNA ligase & Gars & T316 \\
\hline Upregulated & Liprin-alpha-3 & Ppfia3 & S75 \\
\hline Downregulated & Liprin-alpha-3 & Ppfia3 & S682 \\
\hline Upregulated & MARCKS-related protein & Marcksl1 & S104 \\
\hline Upregulated & MARCKS-related protein & Marcksl1 & T85 \\
\hline Upregulated & Microtubule-associated protein & Mapt & S347 \\
\hline Upregulated & Microtubule-associated protein & Mapt & S287 \\
\hline Upregulated & Microtubule-associated protein & Mapt & S344 \\
\hline Upregulated & Microtubule-associated protein & Mapt & S176 \\
\hline Upregulated & Microtubule-associated protein & Mapt & T317 \\
\hline Downregulated & Microtubule-associated protein $1 \mathrm{~A}$ & Map1a Mtap1a & S2207 \\
\hline Downregulated & Microtubule-associated protein $1 \mathrm{~A}$ & Map1a Mtap1a & S2206 \\
\hline Upregulated & Microtubule-associated protein 1B & Map1b & S541 \\
\hline Upregulated & Microtubule-associated protein 1B & Map1b & S561 \\
\hline Downregulated & Microtubule-associated protein 1B & Map1b & T1626 \\
\hline Upregulated & Mitogen-activated protein kinase 1 & Mapk1 Erk2 & Y185 \\
\hline Upregulated & Mitogen-activated protein kinase 1 & Mapk1 Erk2 & T183 \\
\hline Upregulated & Mitogen-activated protein kinase 1 & Mapk1 Erk2 & T179 \\
\hline Upregulated & Mitogen-activated protein kinase 3 & Mapk3 Erk1 & Y205 \\
\hline Upregulated & Mitogen-activated protein kinase 3 & Mapk3 Erk1 & $\mathrm{T} 203$ \\
\hline Downregulated & Myc box-dependent-interacting protein 1 (Amphiphysin II) & Bin1 Amph2 & S332 \\
\hline Downregulated & Myc box-dependent-interacting protein 1 (Amphiphysin II) & Bin1 Amph2 & T328 \\
\hline Upregulated & Myosin-10 & Myh10 & S1952 \\
\hline
\end{tabular}




\begin{tabular}{|c|c|c|c|}
\hline Upregulated & Myosin-10 & Myh10 & S1956 \\
\hline Upregulated & Myristoylated alanine-rich C-kinase substrate & Marcks & S163 \\
\hline Upregulated & Myristoylated alanine-rich C-kinase substrate & Marcks & T143 \\
\hline Upregulated & Myristoylated alanine-rich C-kinase substrate & Marcks & S138 \\
\hline Upregulated & Neurabin-2 & Ppp1r9b & S100 \\
\hline Upregulated & Neurabin-2 & Ppp1r9b & S99 \\
\hline Upregulated & Neuromodulin & Gap43 & S192 \\
\hline Upregulated & Numb-like protein & Numbl Nbl & S411 \\
\hline Upregulated & Plasma membrane calcium-transporting ATPase 4 & Atp2b4 & S1103 \\
\hline Downregulated & Platelet glycoprotein Ib beta chain (Septin 5, isoform CRA_d) & Sept5 & S17 \\
\hline Upregulated & Poliovirus receptor-related 1 & Pvrl1 & S421 \\
\hline Downregulated & Protein Ank2 & Ank2 & S1722 \\
\hline Downregulated & Protein bassoon & Bsn & S241 \\
\hline Downregulated & Protein bassoon & Bsn & S245 \\
\hline Downregulated & Protein bassoon & Bsn & S263 \\
\hline Downregulated & Protein bassoon & Bsn & S265 \\
\hline Downregulated & Protein bassoon & Bsn & S821 \\
\hline Upregulated & Protein bassoon & Bsn & S1220 \\
\hline Downregulated & Protein bassoon & Bsn & S1353 \\
\hline Downregulated & Protein bassoon & Bsn & S1357 \\
\hline Downregulated & Protein bassoon & Bsn & S1475 \\
\hline Downregulated & Protein bassoon & Bsn & T1476 \\
\hline Downregulated & Protein bassoon & Bsn & S1480 \\
\hline Downregulated & Protein bassoon & Bsn & S2016 \\
\hline Downregulated & Protein bassoon & Bsn & S2527 \\
\hline Downregulated & Protein bassoon & Bsn & S2789 \\
\hline Downregulated & Protein bassoon & Bsn & S2792 \\
\hline Upregulated & Protein bassoon & Bsn & S2795 \\
\hline Downregulated & Protein bassoon & Bsn & S2806 \\
\hline Upregulated & Protein bassoon & Bsn & S2842 \\
\hline Upregulated & Protein bassoon & Bsn & S2844 \\
\hline Downregulated & Protein bassoon & Bsn & S3006 \\
\hline Upregulated & Protein bassoon & Bsn & S3497 \\
\hline Upregulated & Protein Dmxl2 & Dmxl2 & T587 \\
\hline Upregulated & Protein Dmxl2 & Dmxl2 & S1586 \\
\hline Upregulated & Protein Dmxl2 & Dmxl2 & S589 \\
\hline Upregulated & Protein Dmxl2 & Dmxl2 & S593 \\
\hline Upregulated & Protein Dmxl2 & Dmxl2 & S1584 \\
\hline Downregulated & Protein Dmxl2 & Dmxl2 & S150 \\
\hline Downregulated & Protein Hecw2 & Hecw2 & S1048 \\
\hline Upregulated & Protein Iqsec1 & Iqsec1 & S74 \\
\hline Downregulated & $\begin{array}{l}\text { Protein kinase } \mathrm{C} \text { and casein kinase substrate in neurons protein } \\
1\end{array}$ & Pacsin 1 & S336 \\
\hline
\end{tabular}




\begin{tabular}{|c|c|c|c|}
\hline Upregulated & Protein LOC100909464 & LOC100909464 & S567 \\
\hline Upregulated & Protein Map2k4 & Map2k4 & S42 \\
\hline Upregulated & Protein Map2k4 & Map2k4 & $\mathrm{T} 40$ \\
\hline Downregulated & Protein Pcp2 & Pcp2 & S92 \\
\hline Downregulated & Protein Pcp2 & Pcp2 & S98 \\
\hline Downregulated & Protein Pcp2 & Pcp2 & S111 \\
\hline Upregulated & Protein piccolo & Pclo & S3632 \\
\hline Upregulated & Protein piccolo & Pclo & S3626 \\
\hline Downregulated & Protein piccolo & Pclo & T111 \\
\hline Downregulated & Protein piccolo & Pclo & S3561 \\
\hline Downregulated & Protein piccolo & Pclo & S3565 \\
\hline Downregulated & Protein piccolo & Pclo & S591 \\
\hline Upregulated & Protein Psd3 & Psd3 & S98 \\
\hline Upregulated & Protein RGD1559864 & RGD1559864 & $\mathrm{T} 47$ \\
\hline Upregulated & Protein Sorcs2 & Sorcs2 & T298 \\
\hline Upregulated & Protein Sorcs2 & Sorcs2 & Y299 \\
\hline Upregulated & Rabphilin-3A & Rph3a & S274 \\
\hline Upregulated & Regulating synaptic membrane exocytosis protein 1 & Rims1 & S346 \\
\hline Upregulated & Regulating synaptic membrane exocytosis protein 1 & Rims1 & S1141 \\
\hline Upregulated & Regulating synaptic membrane exocytosis protein 1 & Rims1 & S991 \\
\hline Upregulated & Regulating synaptic membrane exocytosis protein 1 & Rims1 & S413 \\
\hline Downregulated & Regulating synaptic membrane exocytosis protein 1 & Rims1 & S1045 \\
\hline Downregulated & Regulating synaptic membrane exocytosis protein 1 & Rims1 & S745 \\
\hline Downregulated & Regulating synaptic membrane exocytosis protein 1 & Rims1 & S742 \\
\hline Downregulated & Reticulon-3 & Rtn3 & S31 \\
\hline Upregulated & Serine/threonine-protein kinase BRSK1 & Brsk1 & S508 \\
\hline Upregulated & Serine/threonine-protein kinase MARK1 & Mark1 & S649 \\
\hline Upregulated & $\begin{array}{l}\text { Serine/threonine-protein phosphatase 2B catalytic subunit } \\
\text { alpha isoform }\end{array}$ & Ppp3ca & T458 \\
\hline Upregulated & $\begin{array}{l}\text { Serine/threonine-protein phosphatase } 2 \mathrm{~B} \text { catalytic subunit } \\
\text { alpha isoform }\end{array}$ & Ppp3ca & S459 \\
\hline Upregulated & Sodium/potassium/calcium exchanger 2 & Slc24a2 & S41 \\
\hline Upregulated & Sodium/potassium/calcium exchanger 2 & Slc24a2 & S44 \\
\hline Upregulated & Sodium/potassium/calcium exchanger 2 & Slc24a2 & S42 \\
\hline Downregulated & Sodium-dependent neutral amino acid transporter SLC6A17 & Slc6a17 & S682 \\
\hline Downregulated & Sodium-dependent neutral amino acid transporter SLC6A17 & Slc6a17 & S686 \\
\hline Upregulated & SRC kinase signaling inhibitor 1 & Snip & S941 \\
\hline Upregulated & SRC kinase signaling inhibitor 1 & Snip & S1077 \\
\hline Upregulated & SRC kinase signaling inhibitor 1 & Snip & S1094 \\
\hline Upregulated & SRC kinase signaling inhibitor 1 & Snip & S547 \\
\hline Downregulated & SRC kinase signaling inhibitor 1 & Snip & S527 \\
\hline Upregulated & Stathmin & Stmn1 & S16 \\
\hline Upregulated & Synapsin-1 & Syn1 & S603 \\
\hline
\end{tabular}




\begin{tabular}{|l|l|l|l|}
\hline Upregulated & Synapsin-1 & Syn1 & S566 \\
\hline Upregulated & Synapsin-1 & Syn1 & S447 \\
\hline Downregulated & Synapsin-1 & Syn1 & S436 \\
\hline Downregulated & Synapsin-1 & Syn1 & S662 \\
\hline Downregulated & Synapsin-1 & Syn1 & T434 \\
\hline Downregulated & Synapsin-1 & Syn1 & S432 \\
\hline Downregulated & Synapsin-1 & Syn1 & S62 \\
\hline Downregulated & Synapsin-1 & Syn1 & S579 \\
\hline Downregulated & Synapsin-1 & Syn1 & S39 \\
\hline Downregulated & Synapsin-1 & Syn1 & S508 \\
\hline Upregulated & Synapsin-2 & Syn2 & S524 \\
\hline Upregulated & Synapsin-2 & Syn2 & S530 \\
\hline Upregulated & Synapsin-3 & Syn3 & S265 \\
\hline Upregulated & Syntaxin-6 & Stx6 & S2 \\
\hline Upregulated & Syntaxin-binding protein 5 & Stxbp5 Llgl3 & S1023 \\
\hline Upregulated & Transgelin-3 & Tagln3 Np25 & S163 \\
\hline Upregulated & Tyrosine 3-monooxygenase & Th & S19 \\
\hline Upregulated & Uncharacterized protein & LOC690035 & S355 \\
\hline Upregulated & Vesicle-fusing ATPase & Nsf & S206 \\
\hline Downregulated & Voltage-dependent P/Q-type calcium channel subunit alpha-1A & Cacna1a & S1589 \\
\hline
\end{tabular}

Table 6-2: List of regulated phosphosites in comparison EGTA vs $\mathrm{K}^{+}, \mathrm{Ca}^{2+}$

\begin{tabular}{|l|l|l|l|}
\hline Regulation & Protein names & Gene names & $\begin{array}{l}\text { Aminoacid/ } \\
\text { position }\end{array}$ \\
\hline Upregulated & $\begin{array}{l}\text { 1-phosphatidylinositol 4,5-bisphosphate phosphodiesterase } \\
\text { beta-1 }\end{array}$ & Plcb1 & S1199 \\
\hline Downregulated & ADP-ribosylation factor GTPase-activating protein 1 (ARF GAP & Arfgap1 & S305 \\
\hline Downregulated & A-kinase anchor protein 12 (AKAP-12) & Akap12 & S1466 \\
\hline Downregulated & AP2-associated protein kinase 1 & Aak1 & S672 \\
\hline Downregulated & AP2-associated protein kinase 1 & Aak1 & S670 \\
\hline Downregulated & AP2-associated protein kinase 1 & Aak1 & T675 \\
\hline Downregulated & $\begin{array}{l}\text { Arf-GAP with SH3 domain, ANK repeat and PH domain- } \\
\text { containing protein 1 }\end{array}$ & Asap1 Ddef1 & S973 \\
\hline Upregulated & ATP citrate lyase, isoform CRA_b (ATP-citrate synthase) & Acly & S455 \\
\hline Upregulated & ATP citrate lyase, isoform CRA_b (ATP-citrate synthase) & Acly & T453 \\
\hline Downregulated & Band 4.1-like protein 1 & Epb4111 & S1349 \\
\hline Downregulated & $\begin{array}{l}\text { Calcium/calmodulin-dependent protein kinase type II subunit } \\
\text { delta }\end{array}$ & Camk2d & T337 \\
\hline Downregulated & Calcium-dependent secretion activator 1 & Cadps & S89 \\
\hline Downregulated & cAMP-dependent protein kinase catalytic subunit beta & Prkacb Pkacb & T198 \\
\hline Downregulated & cAMP-dependent protein kinase catalytic subunit beta & Prkacb Pkacb & T196 \\
\hline Upregulated & Caskin-1 & Caskin1 & T815 \\
\hline Downregulated & Caskin-1 & Caskin1 & S1362 \\
\hline
\end{tabular}




\begin{tabular}{|c|c|c|c|}
\hline Upregulated & cGMP-dependent 3',5'-cyclic phosphodiesterase & Pde2a & S900 \\
\hline Downregulated & Clathrin coat assembly protein AP180 & Snap91 & S600 \\
\hline Upregulated & Dihydropyrimidinase-related protein 1 (DRP-1) & Crmp1 Dpysl1 & S542 \\
\hline Upregulated & Dihydropyrimidinase-related protein 2 (DRP-2) & Dpysl2 & S542 \\
\hline Upregulated & Dihydropyrimidinase-related protein 2 (DRP-2) & Dpysl2 & S540 \\
\hline Upregulated & Dihydropyrimidinase-related protein 2 (DRP-2) & Dpysl2 & S517 \\
\hline Upregulated & Dihydropyrimidinase-related protein 2 (DRP-2) & Dpysl2 & T514 \\
\hline Upregulated & Dihydropyrimidinase-related protein 2 (DRP-2) & Dpysl2 & S518 \\
\hline Upregulated & $\begin{array}{l}\text { Discs, large (Drosophila) homolog-associated protein 3, isoform } \\
\text { CRA_b }\end{array}$ & Dlgap3 & S712 \\
\hline Upregulated & $\begin{array}{l}\text { Discs, large (Drosophila) homolog-associated protein 3, isoform } \\
\text { CRA_b }\end{array}$ & Dlgap3 & S185 \\
\hline Downregulated & $\begin{array}{l}\text { Disks large homolog } 2 \text { (Channel-associated protein of synapse- } \\
110 \text { ) }\end{array}$ & Dlg2 Dlgh2 & S360 \\
\hline Upregulated & Disks large-associated protein 2 & Dlgap2 & S977 \\
\hline Downregulated & DnaJ (Hsp40) homolog, subfamily C, member 6 & Dnajc6 & S652 \\
\hline Upregulated & Drebrin-like protein (Actin-binding protein 1) & Dbnl Sh3p7 & S277 \\
\hline Downregulated & Dual specificity mitogen-activated protein kinase kinase 2 & Map2k2 & S222 \\
\hline Upregulated & Dynamin-1 (EC 3.6.5.5) (B-dynamin) (D100) & Dnm1 & S778 \\
\hline Downregulated & Dynamin-1 (EC 3.6.5.5) (B-dynamin) (D100) & Dnm1 & S774 \\
\hline Downregulated & Dynamin-1 & Dnm1 & T776 \\
\hline Downregulated & Dynamin-1 & Dnm1 & S857 \\
\hline Downregulated & Dynamin-1 & Dnm1 & S822 \\
\hline Downregulated & Dynamin-1 & Dnm1 & S817 \\
\hline Downregulated & Dynamin-1 & Dnm1 & S853 \\
\hline Upregulated & Dynamin-3 & Dnm3 Dyn3 & S759 \\
\hline Downregulated & Endophilin-A2 & Sh3gl1 Sh3p8 & S288 \\
\hline Downregulated & ERC protein 2 & Erc2 & S18 \\
\hline Upregulated & Eukaryotic translation initiation factor $4 \mathrm{~B}$ & Eif4b & S497 \\
\hline Upregulated & Eukaryotic translation initiation factor 4B & Eif4b & S498 \\
\hline Upregulated & Eukaryotic translation initiation factor $4 \mathrm{~B}$ & Eif4b & S504 \\
\hline Downregulated & FXYD domain-containing ion transport regulator 7 & Fxyd7 & S60 \\
\hline Downregulated & FXYD domain-containing ion transport regulator 7 & Fxyd7 & S56 \\
\hline Downregulated & FXYD domain-containing ion transport regulator 7 & Fxyd7 & S58 \\
\hline Upregulated & Glutamate decarboxylase 1 & Gad1 Gad67 & T53 \\
\hline Upregulated & Glutamate decarboxylase 1 & Gad1 Gad67 & S55 \\
\hline Upregulated & Glycine--tRNA ligase & Gars & T316 \\
\hline Upregulated & GTPase activating RANGAP domain-like 4 & Rap1gap2 & S324 \\
\hline Upregulated & Intersectin-1 & Itsn1 & S975 \\
\hline Upregulated & Intersectin-1 & Itsn1 & S623 \\
\hline Upregulated & Kinesin light chain 2 & Klc2 & S581 \\
\hline Upregulated & Kinesin light chain 2 & Klc2 & S582 \\
\hline Downregulated & Liprin-alpha-3 & Ppfia3 & S682 \\
\hline
\end{tabular}




\begin{tabular}{|c|c|c|c|}
\hline Downregulated & MAGUK p55 subfamily member 3 & $\begin{array}{l}\text { Mpp3 Dlg3 } \\
\text { Dusp3 }\end{array}$ & S354 \\
\hline Upregulated & MARCKS-related protein & Marcksl1 Mlp & S104 \\
\hline Upregulated & Microtubule-associated protein & Mapt & S347 \\
\hline Upregulated & Microtubule-associated protein & Mapt & S287 \\
\hline Upregulated & Microtubule-associated protein & Mapt & S176 \\
\hline Upregulated & Microtubule-associated protein & Mapt & T179 \\
\hline Upregulated & Microtubule-associated protein & Mapt & S327 \\
\hline Upregulated & Microtubule-associated protein 1B & Map1b & S541 \\
\hline Downregulated & Microtubule-associated protein 1B & Map1b & T1626 \\
\hline Upregulated & Microtubule-associated protein 4 (MAP-4) & Map4 & S904 \\
\hline Upregulated & Mitochondrial import receptor subunit TOM20 homolog & Tomm20 & S138 \\
\hline Upregulated & Mitogen-activated protein kinase 1 & Mapk1 Erk2 & Y185 \\
\hline Upregulated & Mitogen-activated protein kinase 1 & Mapk1 Erk2 & T183 \\
\hline Upregulated & Mitogen-activated protein kinase 1 & Mapk1 Erk2 & T188 \\
\hline Upregulated & Mitogen-activated protein kinase 1 & Mapk1 Erk2 & T179 \\
\hline Upregulated & Mitogen-activated protein kinase 3 & Mapk3 Erk1 & Y205 \\
\hline Upregulated & Mitogen-activated protein kinase 3 & Mapk3 Erk1 & T203 \\
\hline Downregulated & Myc box-dependent-interacting protein 1 (Amphiphysin II) & Bin1 Amph2 & S332 \\
\hline Downregulated & Myc box-dependent-interacting protein 1 (Amphiphysin II) & Bin1 Amph2 & T328 \\
\hline Downregulated & $\begin{array}{l}\text { Myosin, heavy polypeptide 10, non-muscle, isoform CRA_b } \\
\text { (Myosin-10) }\end{array}$ & Myh10 & S1956 \\
\hline Upregulated & Myristoylated alanine-rich C-kinase substrate & Marcks & S163 \\
\hline Upregulated & Neurabin-2 (Neurabin-II) & Ppp1r9b & S99 \\
\hline Upregulated & Oxidation resistance protein 1 (Fragment) & Oxr1 & T178 \\
\hline Downregulated & Peripheral plasma membrane protein CASK & Cask & S24 \\
\hline Downregulated & Phosphatase and actin regulator & Phactr1 & S67 \\
\hline Downregulated & Phosphatidylinositol 4-phosphate 5-kinase type-1 gamma & Pip5k1c & S475 \\
\hline Downregulated & Platelet glycoprotein Ib beta chain & Gp1bb Sept5 & S234 \\
\hline Downregulated & Platelet glycoprotein Ib beta chain (Septin 5, isoform CRA_d) & Sept5 Gp1bb & S17 \\
\hline Downregulated & Protein Ank2 & Ank2 & S1722 \\
\hline Upregulated & Protein Arhgap1 & Arhgap1 & S27 \\
\hline Upregulated & Protein bassoon & Bsn & S3497 \\
\hline Upregulated & Protein bassoon & Bsn & S2795 \\
\hline Upregulated & Protein bassoon & Bsn & S1220 \\
\hline Upregulated & Protein bassoon & Bsn & S2632 \\
\hline Downregulated & Protein bassoon & Bsn & S2004 \\
\hline Downregulated & Protein bassoon & Bsn & S3501 \\
\hline Downregulated & Protein bassoon & Bsn & S1357 \\
\hline Downregulated & Protein bassoon & Bsn & S3006 \\
\hline Downregulated & Protein bassoon & Bsn & T1476 \\
\hline Downregulated & Protein bassoon & Bsn & S235 \\
\hline Downregulated & Protein bassoon & Bsn & S2806 \\
\hline
\end{tabular}




\begin{tabular}{|c|c|c|c|}
\hline Downregulated & Protein bassoon & Bsn & S265 \\
\hline Downregulated & Protein bassoon & Bsn & S263 \\
\hline Downregulated & Protein Cpne6 & Cpne6 & S68 \\
\hline Upregulated & Protein Dmxl2 & Dmxl2 & S589 \\
\hline Upregulated & Protein Dmxl2 & Dmxl2 & S1584 \\
\hline Upregulated & Protein FAM131B & Fam131b & S102 \\
\hline Downregulated & Protein Fam57b (Putative uncharacterized protein & Fam57b & S269 \\
\hline Upregulated & Protein Iqsec1 & Iqsec1 & S74 \\
\hline Upregulated & Protein Iqsec1 & Iqsec1 & S165 \\
\hline Downregulated & Protein kinase $\mathrm{C}$ & Prkca & T514 \\
\hline Downregulated & Protein kinase $\mathrm{C}$ & Prkcd & S504 \\
\hline Downregulated & Protein kinase $\mathrm{C}$ & Prkcd & T505 \\
\hline Upregulated & $\begin{array}{l}\text { Protein kinase } \mathrm{C} \text { and casein kinase substrate in neurons } \\
\text { protein1 }\end{array}$ & Pacsin1 & S343 \\
\hline Upregulated & Protein LOC100910792 & Amph & S239 \\
\hline Upregulated & Protein LOC100911610 & LOC100911610 & T427 \\
\hline Upregulated & Protein Map2k4 & Map2k4 & S42 \\
\hline Upregulated & Protein Mon2 & Mon2 & S1167 \\
\hline Upregulated & Protein NDRG2 & Ndrg2 & S318 \\
\hline Upregulated & Protein NDRG2 & Ndrg2 & S324 \\
\hline Upregulated & Protein NDRG2 & Ndrg2 & T316 \\
\hline Downregulated & Protein Pcp2 (Purkinje cell protein 2 (L7), isoform CRA_c) & Pcp2 & S111 \\
\hline Downregulated & Protein Pcp2 (Purkinje cell protein 2 (L7), isoform CRA_c) & Pcp2 & S92 \\
\hline Downregulated & Protein Pcp2 (Purkinje cell protein 2 (L7), isoform CRA_c) & Pcp2 & T90 \\
\hline Upregulated & Protein phosphatase 1H (EC 3.1.3.16) & Ppm1h & S220 \\
\hline Downregulated & Protein phosphatase methylesterase 1 (PME-1) (EC 3.1.1.-) & Ppme1 & S243 \\
\hline Downregulated & Protein phosphatase methylesterase 1 (PME-1) (EC 3.1.1.-) & Ppme1 & $\mathrm{T} 242$ \\
\hline Downregulated & Protein piccolo (Aczonin) & Pclo & S1353 \\
\hline Downregulated & Protein piccolo (Aczonin) & Pclo & S591 \\
\hline Downregulated & Protein Rabl6 (Putative uncharacterized protein & Rabl6 & S489 \\
\hline Upregulated & Protein RGD1559864 & RGD1559864 & S43 \\
\hline Upregulated & Protein RGD1559864 & RGD1559864 & T47 \\
\hline Downregulated & Protein RGD1559864 & RGD1559864 & S309 \\
\hline Downregulated & Protein Rras2 (RCG40097) & Rras2 & S186 \\
\hline Downregulated & Protein Smap2 (Smap2 protein) & Smap2 & S219 \\
\hline Upregulated & Protein Sorcs2 & Sorcs2 & T298 \\
\hline Upregulated & Protein Sorcs2 & Sorcs2 & Y299 \\
\hline Upregulated & Rab GDP dissociation inhibitor alpha (Rab GDI alpha) & Gdi1 & T157 \\
\hline Upregulated & Rabphilin-3A & Rph3a & S274 \\
\hline Upregulated & Regulating synaptic membrane exocytosis protein 1 & Rims1 & S346 \\
\hline Upregulated & Regulating synaptic membrane exocytosis protein 1 & Rims1 & S95 \\
\hline Upregulated & Regulating synaptic membrane exocytosis protein 1 & Rims1 & S991 \\
\hline Upregulated & Regulating synaptic membrane exocytosis protein 1 & Rims1 & S413 \\
\hline
\end{tabular}




\begin{tabular}{|c|c|c|c|}
\hline Upregulated & Regulating synaptic membrane exocytosis protein 2 & Rims2 & S369 \\
\hline Downregulated & Serine/threonine-protein kinase BRSK2 (Fragment) & Brsk2 & S91 \\
\hline Upregulated & Serine/threonine-protein kinase MARK1 (Fragment) & Mark1 & S649 \\
\hline Downregulated & Serine/threonine-protein kinase MARK1 (Fragment) & Mark1 & T198 \\
\hline Upregulated & $\begin{array}{l}\text { Serine/threonine-protein phosphatase 2B catalytic subunit } \\
\text { alpha isoform }\end{array}$ & Ppp3ca & T458 \\
\hline Downregulated & $\begin{array}{l}\text { SH3-containing GRB2-like protein 3-interacting protein } 1 \\
\text { (Endophilin-3-interacting protein) }\end{array}$ & Sgip1 & T263 \\
\hline Downregulated & Sn1-specific diacylglycerol lipase alpha & Dagla & S808 \\
\hline Upregulated & Sodium/potassium/calcium exchanger 2 & Slc24a2 & S44 \\
\hline Upregulated & Sodium/potassium/calcium exchanger 2 & Slc24a2 & S41 \\
\hline Downregulated & Sodium-dependent neutral amino acid transporter SLC6A17 & Slc6a17 & S665 \\
\hline Downregulated & Sodium-dependent neutral amino acid transporter SLC6A17 & Slc6a17 & S686 \\
\hline Downregulated & Sodium-driven chloride bicarbonate exchanger & Slc4a10 & S296 \\
\hline Upregulated & SRC kinase signaling inhibitor 1 (SNIP) & Srcin1 & S941 \\
\hline Upregulated & SRC kinase signaling inhibitor 1 (SNIP) & Srcin 1 & S1077 \\
\hline Upregulated & stathmin 1 & STMN1 & S16 \\
\hline Upregulated & STE20/SPS1-related proline-alanine-rich protein kinase & Stk39 & T363 \\
\hline Upregulated & Synapsin-1 (Synapsin I) & Syn1 & S603 \\
\hline Upregulated & Synapsin-1 (Synapsin I) & Syn1 & S566 \\
\hline Upregulated & Synapsin-1 (Synapsin I) & Syn1 & S447 \\
\hline Downregulated & Synapsin-1 (Synapsin I) & Syn1 & S436 \\
\hline Downregulated & Synapsin-1 (Synapsin I) & Syn1 & $\mathrm{T} 434$ \\
\hline Downregulated & Synapsin-1 (Synapsin I) & Syn1 & S432 \\
\hline Downregulated & Synapsin-1 (Synapsin I) & Syn1 & S579 \\
\hline Downregulated & Synapsin-1 (Synapsin I) & Syn1 & S62 \\
\hline Downregulated & Synapsin-1 (Synapsin I) & Syn1 & S508 \\
\hline Downregulated & Synapsin-1 (Synapsin I) & Syn1 & S39 \\
\hline Upregulated & Synapsin-3 & Syn3 & S265 \\
\hline Downregulated & Synaptojanin-1 & Synj1 & S1053 \\
\hline Downregulated & Synaptojanin-1 & Synj1 & T1048 \\
\hline Upregulated & Synaptotagmin-12 & Syt12 & S99 \\
\hline Upregulated & Syntaxin-6 & Stx6 & S2 \\
\hline Upregulated & Syntaxin-binding protein 5 (Tomosyn-1) & Stxbp5 Llgl3 & S1023 \\
\hline Upregulated & Thymosin beta-4 & Tmsb4x & $\mathrm{T} 21$ \\
\hline Upregulated & Transcriptional activator protein Pur-alpha & Pura & T164 \\
\hline Upregulated & Transcriptional activator protein Pur-alpha & Pura & S163 \\
\hline Upregulated & Transgelin-3 (Neuronal protein 22) (NP22) & Tagln3 Np25 & S163 \\
\hline Downregulated & Voltage-dependent P/Q-type calcium channel subunit alpha-1A & Cacna1a & S1589 \\
\hline
\end{tabular}


Table 6-3: List of regulated phosphosites in comparison EGTA vs $\mathrm{K}^{+}$, EGTA

\begin{tabular}{|c|c|c|c|}
\hline Regulation & Protein names & Gene names & $\begin{array}{l}\text { Aminoacid/ } \\
\text { Position }\end{array}$ \\
\hline Downregulated & Protein Sept4 & Sep4 & S306 \\
\hline Upregulated & AP2-associated protein kinase 1 & Aak1 & S678 \\
\hline Downregulated & A-kinase anchor protein 12 (AKAP-12) & Akap12 & S509 \\
\hline Downregulated & A-kinase anchor protein 12 (AKAP-12) & Akap12 & S511 \\
\hline Upregulated & Protein Ank2 & Ank2 & S1816 \\
\hline Upregulated & AP-3 complex subunit delta & Ap3d1 & S754 \\
\hline Upregulated & Aquaporin-4 (AQP-4) (Mercurial-insensitive water channel) & Aqp4 & S221 \\
\hline Downregulated & Protein Arhgap1 & Arhgap1 & S50 \\
\hline Downregulated & Protein Arhgap1 & Arhgap1 & S51 \\
\hline Downregulated & Protein Armc4 & Armc4 & S304 \\
\hline Downregulated & Protein Armc4 & Armc4 & S308 \\
\hline Downregulated & Sodium/potassium-transporting ATPase subunit alpha-2 & Atp1a2 & S439 \\
\hline Upregulated & Protein bassoon & Bsn & S3006 \\
\hline Upregulated & Protein bassoon & Bsn & S2527 \\
\hline Upregulated & Protein bassoon & Bsn & S2054 \\
\hline Upregulated & Voltage-dependent R-type calcium channel subunit alpha-1E & Cacna1e & S15 \\
\hline Upregulated & $\begin{array}{l}\text { Calcium channel, voltage-dependent, beta } 1 \text { subunit, isoform } \\
\text { CRA_a }\end{array}$ & Cacnb1 & S417 \\
\hline Downregulated & $\begin{array}{l}\text { Calcium/calmodulin-dependent protein kinase type II subunit } \\
\text { alpha }\end{array}$ & Camk2a & T286 \\
\hline Upregulated & $\begin{array}{l}\text { Calcium/calmodulin-dependent protein kinase II, beta, isoform } \\
\text { CRA_a }\end{array}$ & Camk2b & T401 \\
\hline Upregulated & Claudin & Cldn11 & S194 \\
\hline Downregulated & DnaJ (Hsp40) homolog, subfamily C, member 6 & Dnajc6 & S568 \\
\hline Upregulated & Dynamin-1 & Dnm1 Dnm & S822 \\
\hline Downregulated & FXYD domain-containing ion transport regulator 7 & Fxyd7 & S58 \\
\hline Downregulated & Glycine--tRNA ligase & Gars & T316 \\
\hline Upregulated & Guanine nucleotide-binding protein G(o) subunit alpha & Gnao1 Gna0 & S207 \\
\hline Downregulated & Platelet glycoprotein Ib beta chain & Gp1bb Sept5 & $\mathrm{T} 13$ \\
\hline Upregulated & Probable G-protein-coupled receptor 158 & Gpr158 & S946 \\
\hline Downregulated & Glutamate receptor ionotropic, delta-2 & Grid2 & S816 \\
\hline Downregulated & Glutamate receptor ionotropic, delta-2 & Grid2 & S809 \\
\hline Downregulated & Heat shock protein HSP 90-beta & Hsp90ab1 & S255 \\
\hline Downregulated & Heat shock protein HSP 90-beta & Hsp90ab1 & S261 \\
\hline Upregulated & Potassium voltage-gated channel subfamily C member 1 & Kcnc1 & S160 \\
\hline Upregulated & Calcium-activated potassium channel subunit alpha-1 & Kcnma1 Kcnma & T1001 \\
\hline Downregulated & Kinesin light chain 2 & Klc2 & S581 \\
\hline Downregulated & Kinesin light chain 2 & Klc2 & S151 \\
\hline Upregulated & Protein LOC100365676 & LOC100365676 & S114 \\
\hline Downregulated & Protein LOC100911356 & LOC100911356 & S35 \\
\hline Downregulated & Protein LOC100911356 & LOC100911356 & S39 \\
\hline Downregulated & Leucine-rich repeat-containing protein 4B & Lrrc4b Lrig4 & S700 \\
\hline Downregulated & Microtubule-associated protein 1B & Map1b & S614 \\
\hline Downregulated & Microtubule-associated protein 1B & Map1b & S561 \\
\hline Downregulated & Microtubule-associated protein & Map2 & S1051 \\
\hline Downregulated & Myristoylated alanine-rich C-kinase substrate & Marcks & S163 \\
\hline
\end{tabular}




\begin{tabular}{|c|c|c|c|}
\hline Downregulated & MARCKS-related protein & Marcksl1 Mlp & T85 \\
\hline Downregulated & MARCKS-related protein & Marcksl1 Mlp & S22 \\
\hline Upregulated & Unconventional myosin-Va & Myo5a & S1625 \\
\hline Upregulated & NCK interacting protein with SH3 domain & Nckipsd & S122 \\
\hline Downregulated & Uncharacterized protein & Nefm & S641 \\
\hline Downregulated & NSFL1 cofactor $\mathrm{p} 47$ & Nsfl1c & S114 \\
\hline Upregulated & Proliferation-associated protein 2G4 & Pa2g4 & $\mathrm{S} 2$ \\
\hline Downregulated & Protein piccolo (Aczonin) & Pclo & S3565 \\
\hline Downregulated & Protein piccolo (Aczonin) & Pclo & S617 \\
\hline Upregulated & Protein piccolo (Aczonin) & Pclo & S4340 \\
\hline Downregulated & ATP-dependent 6-phosphofructokinase, platelet type & Pfkp & S2 \\
\hline Downregulated & $\begin{array}{l}\text { 1-phosphatidylinositol 4,5-bisphosphate phosphodiesterase } \\
\text { beta-1 }\end{array}$ & Plcb1 & S1199 \\
\hline Downregulated & $\begin{array}{l}\text { 1-phosphatidylinositol 4,5-bisphosphate phosphodiesterase } \\
\text { beta-1 }\end{array}$ & Plcb1 & S1197 \\
\hline Upregulated & Neurabin-2 (Neurabin-II) & Ppp1r9b & S100 \\
\hline Downregulated & Protein kinase C (EC 2.7.11.13) & Prkcd & S504 \\
\hline Downregulated & Protein kinase C (EC 2.7.11.13) & Prkce & S349 \\
\hline Upregulated & Protein Prrt3 & Prrt3 & S807 \\
\hline Downregulated & Protein Psd3 & Psd3 & S76 \\
\hline Upregulated & Protein Psd3 & Psd3 & S337 \\
\hline Downregulated & Rab3 GTPase-activating protein non-catalytic subunit & Rab3gap2 & S877 \\
\hline Upregulated & Protein Rabl6 & Rabl6 & S472 \\
\hline Upregulated & Protein Rabl6 & Rabl6 & S474 \\
\hline Upregulated & Protein Rabl6 & Rabl6 & S475 \\
\hline Downregulated & Protein RGD1559864 & RGD1559864 & T47 \\
\hline Upregulated & Regulating synaptic membrane exocytosis protein 1 & Rims1 & S991 \\
\hline Upregulated & Reticulon & Rtn4 & S16 \\
\hline Downregulated & Secretogranin 2, isoform CRA_a & Scg2 & S531 \\
\hline Downregulated & Secretogranin 2, isoform CRA_a & Scg2 & $\mathrm{T} 274$ \\
\hline Upregulated & Signal-induced proliferation-associated 1-like protein 1 & Sipa1l1 & S1129 \\
\hline Downregulated & Monocarboxylate transporter 1 (MCT 1) & Slc16a1 Mct1 & S210 \\
\hline Downregulated & Monocarboxylate transporter 1 (MCT 1) & Slc16a1 Mct1 & S213 \\
\hline Upregulated & $\begin{array}{l}\text { Vesicular inhibitory amino acid transporter (GABA and glycine } \\
\text { transporter) }\end{array}$ & $\begin{array}{l}\text { Slc32a1 Vgat } \\
\text { Viaat }\end{array}$ & S95 \\
\hline Upregulated & $\begin{array}{l}\text { Vesicular inhibitory amino acid transporter (GABA and glycine } \\
\text { transporter) }\end{array}$ & $\begin{array}{l}\text { Slc32a1 Vgat } \\
\text { Viaat }\end{array}$ & S97 \\
\hline Upregulated & Sodium/calcium exchanger 2 & Slc8a2 & S713 \\
\hline Downregulated & Protein Sorcs2 & Sorcs2 & T298 \\
\hline Downregulated & Protein Sorcs2 & Sorcs2 & Y299 \\
\hline Downregulated & Stathmin & Stmn3 & S81 \\
\hline Upregulated & RCG61894, isoform CRA_a (Striatin) & Strn & S245 \\
\hline Upregulated & Synapsin-1 (Synapsin I) & Syn1 & S603 \\
\hline Upregulated & Synapsin-1 (Synapsin I) & Syn1 & S39 \\
\hline Upregulated & Synapsin-3 & Syn3 & S700 \\
\hline Upregulated & Synapsin-3 & Syn3 & S697 \\
\hline Upregulated & Ras/Rap GTPase-activating protein SynGAP & Syngap1 & T70 \\
\hline Upregulated & Protein Tanc2 & Tanc2 & S400 \\
\hline Upregulated & Protein Tanc2 & Tanc2 & S404 \\
\hline Downregulated & Protein Tppp & Tppp & T15 \\
\hline
\end{tabular}




\begin{tabular}{|l|l|l|l|}
\hline Downregulated & $\begin{array}{l}\text { Transient receptor potential cation channel subfamily V } \\
\text { member 2 }\end{array}$ & Trpv2 & S15 \\
\hline Downregulated & $\begin{array}{l}\text { Tubulin alpha-1B chain (Alpha-tubulin 2) (Tubulin alpha-2 } \\
\text { chain) }\end{array}$ & Tuba1b Tuba2 & S158 \\
\hline Upregulated & Ubiquitin carboxyl-terminal hydrolase & Usp27x & T149 \\
\hline Upregulated & Vesicle-associated membrane protein 1 & Vamp1 & S62 \\
\hline Downregulated & Vesicle-associated membrane protein 2 & Vamp2 & S74 \\
\hline Upregulated & Vesicle-associated membrane protein 2 & Vamp2 & S60 \\
\hline
\end{tabular}

Table 6-4: List of upregulated phosphosites upon Calcineurin inhibition (putative Calcineurin substrates).

\begin{tabular}{|c|c|c|}
\hline Protein name & Gene name & $\begin{array}{l}\text { Aminoacid// } \\
\text { Position }\end{array}$ \\
\hline Beta-adducin & Add2 & S618 \\
\hline Ankyrin-2 & Ank2 & S1725 \\
\hline Ankyrin-3 & Ank2 & S1702 \\
\hline Rho GTPase-activating protein 1 & Arhgap1 & S51 \\
\hline Autophagy-related protein 2 homolog B & Atg2b & S255 \\
\hline Protein bassoon & Bsn & S3367 \\
\hline Protein bassoon & Bsn & S821 \\
\hline Protein bassoon & Bsn & S1357 \\
\hline Protein bassoon & Bsn & S2806 \\
\hline Protein bassoon & Bsn & S245 \\
\hline Protein bassoon & Bsn & S2792 \\
\hline Protein bassoon & Bsn & S2795 \\
\hline Protein bassoon & Bsn & S3501 \\
\hline Protein bassoon & Bsn & S3504 \\
\hline Protein bassoon & Bsn & S263 \\
\hline Protein bassoon & Bsn & S1473 \\
\hline Protein bassoon & Bsn & S241 \\
\hline Protein bassoon & Bsn & S245 \\
\hline Protein bassoon & Bsn & S2792 \\
\hline Protein bassoon & Bsn & S2795 \\
\hline Protein bassoon & Bsn & T1086 \\
\hline Voltage-dependent P/Q-type calcium channel subunit alpha-1A & Cacna1a & S1981 \\
\hline Voltage-dependent N-type calcium channel subunit alpha-1B & Cacna1b & S745 \\
\hline Voltage-dependent $\mathrm{N}$-type calcium channel subunit alpha-1E & Cacna1e & S15 \\
\hline Calcium/calmodulin-dependent protein kinase type II subunit alpha & Camk2a & S279 \\
\hline Calcium/calmodulin-dependent protein kinase type II subunit beta & Camk2b & S397 \\
\hline Calcium/calmodulin-dependent protein kinase type II subunit beta & Camk2b & T400 \\
\hline Calcium/calmodulin-dependent protein kinase type II subunit beta & Camk2b & $\mathrm{T} 401$ \\
\hline Catenin delta-2 & Ctnnd2 & S224 \\
\hline Src substrate cortactin & Cttn & T327 \\
\hline Disks large homolog 2 & Dlg2 & S365 \\
\hline
\end{tabular}




\begin{tabular}{|c|c|c|}
\hline DmX-like protein 2 & Dmxl2 & S119 \\
\hline Putative tyrosine-protein phosphatase auxilin & Dnajc6 & S568 \\
\hline Dynamin-1 & Dnm1 & S774 \\
\hline Dynamin-1 & Dnm1 & S778 \\
\hline Elongation factor 1-delta & Eef1d & S133 \\
\hline Band 4.1-like protein 3 & Epb4113 & S724 \\
\hline Band 4.1-like protein 3 & Epb4113 & S978 \\
\hline Protein Etl4 & Etl4 & S341 \\
\hline Mitochondrial inner membrane protein & Immt & S340 \\
\hline Kalirin & Kalrn & S401 \\
\hline MAP kinase-activating death domain protein & Madd & S1225 \\
\hline MAP kinase-activating death domain protein & Madd & S1038 \\
\hline Microtubule-associated protein 1A;MAP1A heavy chain;MAP1 light chain LC2 & Map1a & T504 \\
\hline Microtubule-associated protein 1B;MAP1B heavy chain;MAP1 light chain LC1 & Map1b & S1252 \\
\hline Microtubule-associated protein 1B;MAP1B heavy chain;MAP1 light chain LC1 & Map1b & S1257 \\
\hline Microtubule-associated protein 1B;MAP1B heavy chain;MAP1 light chain LC1 & Map1b & S1778 \\
\hline MAP7 domain-containing protein 1 & Map7d1 & S118 \\
\hline Microtubule-associated protein;Microtubule-associated protein tau & Mapt & S461 \\
\hline Microtubule-associated protein;Microtubule-associated protein tau & Mapt & T460 \\
\hline Neural cell adhesion molecule 1 & Ncam1 & T787 \\
\hline Neurofilament heavy polypeptide & Nefh & S592 \\
\hline Protein piccolo & Pclo & S3565 \\
\hline Protein piccolo & Pclo & S1353 \\
\hline Protein piccolo & Pclo & S1451 \\
\hline Protein piccolo & Pclo & S4615 \\
\hline Protein piccolo & Pclo & S3260 \\
\hline Protein piccolo & Pclo & S1841 \\
\hline Protein piccolo & Pclo & S3632 \\
\hline Protein piccolo & Pclo & S591 \\
\hline Protein piccolo & Pclo & S1778 \\
\hline Protein piccolo & Pclo & S1784 \\
\hline Protein piccolo & Pclo & T1772 \\
\hline Myelin proteolipid protein & Plp1 & S114 \\
\hline Liprin-alpha-3 & Ppfia3 & S682 \\
\hline Phosphatidylserine decarboxylase proenzyme 3 & Psd3 & S76 \\
\hline Very-long-chain (3R)-3-hydroxyacyl-CoA dehydratase 3 & Ptplad1 & S114 \\
\hline Rap1 GTPase-activating protein 1 & Rap1gap & S127 \\
\hline Regulating synaptic membrane exocytosis protein 1 & Rims1 & S745 \\
\hline Voltage-dependent anion-selective channel protein 2 & Vdac2 & S116 \\
\hline Regulating synaptic membrane exocytosis protein 1 & Rims1 & S895 \\
\hline Synapsin-1 & Syn1 & S508 \\
\hline VPS10 domain-containing receptor SorCS2 & Sorcs2 & T298 \\
\hline
\end{tabular}




\begin{tabular}{|l|l|l|}
\hline VPS10 domain-containing receptor SorCS2 & Sorcs2 & Y299 \\
\hline Clathrin coat assembly protein AP180 & Snap91 & S300 \\
\hline Protein RGD1306271 & RGD1306271 & S1610 \\
\hline Syntaxin-binding protein 1 & Stxbp1 & S516 \\
\hline SH3 and multiple ankyrin repeat domains protein 1 & Shank1 & S1442 \\
\hline Synapsin-1 & Syn1 & S549 \\
\hline Synapsin-1 & Syn1 & S551 \\
\hline Sorbin and SH3 domain-containing protein 1 & Sorbs1 & S62 \\
\hline Regulating synaptic membrane exocytosis protein 1 & Rims1 & S742 \\
\hline Saccharopine dehydrogenase-like oxidoreductase & Sccpdh & S217 \\
\hline Spectrin beta chain, non-erythrocytic 1 & Sptbn1 & S2158 \\
\hline Proton myo-inositol cotransporter & Slc2a13 & S629 \\
\hline Protein unc-13 homolog A & Unc13a & S255 \\
\hline Endophilin-A2 & Sh3gl1 & S288 \\
\hline Synapsin-1 & Syn1 & S436 \\
\hline NAD-dependent protein deacetylase sirtuin-2 & Sirt2 & S334 \\
\hline SRC kinase signaling inhibitor 1 & Srcin1 & S900 \\
\hline Spectrin beta chain, non-erythrocytic 1 & Sptbn1 & S2162 \\
\hline
\end{tabular}

Table 6-5: List of downregulated phosphosites upon Calcineurin inhibition.

\begin{tabular}{|l|l|l|}
\hline Protein name & Gene name & $\begin{array}{l}\text { Aminoacid/ } \\
\text { Position }\end{array}$ \\
\hline Rho GTPase-activating protein 39 & Arhgap39 & S599 \\
\hline Sodium/potassium-transporting ATPase subunit alpha-2 & Atp1a2 & S439 \\
\hline Plasma membrane calcium-transporting ATPase 4 & Atp2b4 & S1103 \\
\hline Protein bassoon & Bsn & T2580 \\
\hline C2 domain-containing protein 2-like & C2cd2l & S662 \\
\hline Calcium/calmodulin-dependent protein kinase type II subunit gamma & Camk2g & S311 \\
\hline Calcium/calmodulin-dependent protein kinase type II subunit gamma & Camk2g & S321 \\
\hline Calcium/calmodulin-dependent protein kinase type II subunit gamma & Camk2g & S325 \\
\hline Calnexin & Canx & S553 \\
\hline Calnexin & Canx & S563 \\
\hline Cofilin-1 & Cf11 & S3 \\
\hline Choline O-acetyltransferase & Chat & S365 \\
\hline Complexin-1 & Cplx1 & S93 \\
\hline Dihydropyrimidinase-related protein 1 & Crmp1 & S542 \\
\hline Putative tyrosine-protein phosphatase auxilin & Dnajc6 & S564 \\
\hline Putative tyrosine-protein phosphatase auxilin & Dnajc6 & S568 \\
\hline Dynamin-1 & Dnm1 & S817 \\
\hline Dihydropyrimidinase-related protein 2 & Dpysl2 & S542 \\
\hline Dihydropyrimidinase-related protein 2 & Dpysl2 & S518 \\
\hline Dihydropyrimidinase-related protein 2 & Dpysl2 & T514 \\
\hline
\end{tabular}




\begin{tabular}{|c|c|c|}
\hline Dihydropyrimidinase-related protein 3 & Dpysl3 & T509 \\
\hline Epsin-1 & Epn1 & S453 \\
\hline F-box only protein 6 & Fbxo6 & S278 \\
\hline Neuromodulin & Gap43 & S142 \\
\hline Potassium/sodium hyperpolarization-activated cyclic nucleotide-gated channel 2 & Hen2 & S812 \\
\hline Histone H1.4 & Hist1h1e & S2 \\
\hline Heat shock protein HSP 90-alpha & Hsp90aa1 & S263 \\
\hline Protein Klhl34 & Klhl34 & T209 \\
\hline Protein Klhl34 & Klhl34 & $\mathrm{T} 210$ \\
\hline Ubiquitin carboxyl-terminal hydrolase & LOC100911959 & S783 \\
\hline Ubiquitin carboxyl-terminal hydrolase & LOC100911959 & T623 \\
\hline Microtubule-associated protein 1B & Map1b & S930 \\
\hline Mitogen-activated protein kinase 1 & Mapk1 & T183 \\
\hline Mitogen-activated protein kinase 1 & Mapk1 & T183 \\
\hline Mitogen-activated protein kinase 1 & Mapk1 & Y185 \\
\hline Mitogen-activated protein kinase 10 & Mapk10;Mapk8 & T183 \\
\hline Mitogen-activated protein kinase 10 & Mapk10;Mapk8 & Y185 \\
\hline Microtubule-associated protein & Mapt & S473 \\
\hline Microtubule-associated protein & Mapt & S413 \\
\hline Microtubule-associated protein & Mapt & S453 \\
\hline Microtubule-associated protein & Mapt & S255 \\
\hline Microtubule-associated protein & Mapt & S453 \\
\hline Microtubule-associated protein & Mapt & S457 \\
\hline Microtubule-associated protein & Mapt & T460 \\
\hline Myristoylated alanine-rich C-kinase substrate & Marcks & S138 \\
\hline Myotubularin-related protein 9 & Mtmr9 & S498 \\
\hline Myotubularin-related protein 9 & Mtmr9 & T491 \\
\hline Protein NDRG2 & Ndrg2 & S318 \\
\hline Protein NDRG2 & Ndrg2 & S324 \\
\hline Oxidation resistance protein 1 & Oxr1 & S177 \\
\hline Oxidation resistance protein 1 & Oxr1 & S179 \\
\hline Proliferation-associated protein 2G4 & $\mathrm{Pa} 2 \mathrm{~g} 4$ & S2 \\
\hline Protein kinase $\mathrm{C}$ and casein kinase substrate in neurons protein 1 & Pacsin 1 & S343 \\
\hline Protein kinase $\mathrm{C}$ and casein kinase substrate in neurons protein 1 & Pacsin1 & S345 \\
\hline Protein kinase $\mathrm{C}$ and casein kinase substrate in neurons protein 1 & Pacsin 1 & S346 \\
\hline Pyruvate dehydrogenase E1 component subunit alpha, somatic form & Pdha1l1;Pdha1 & S232 \\
\hline 1-phosphatidylinositol 4,5-bisphosphate phosphodiesterase eta-2 & Plch2 & S654 \\
\hline Phosphomannomutase 1 & Pmm1 & S242 \\
\hline Protein phosphatase 1 regulatory subunit 7 & Ppp1r7 & S24 \\
\hline Protein phosphatase 1 regulatory subunit 7 & Ppp1r7 & S27 \\
\hline Serine/threonine-protein phosphatase 2B catalytic subunit alpha isoform & Ppp3ca & S469 \\
\hline Proline-rich transmembrane protein 2 & Prrt2 & T74 \\
\hline
\end{tabular}




\begin{tabular}{|c|c|c|}
\hline Receptor-type tyrosine-protein phosphatase N2 & Ptprn2 & S424 \\
\hline Calcipressin-1 & Rcan1;Rcan3 & S108 \\
\hline Calcipressin-1 & Rcan1;Rcan3 & S112 \\
\hline Protein Phf24 & RGD1559864 & $\mathrm{T} 47$ \\
\hline SH3-containing GRB2-like protein 3-interacting protein 1 & Sgip1 & S265 \\
\hline SH3-containing GRB2-like protein 3-interacting protein 1 & Sgip1 & T263 \\
\hline Sodium/hydrogen exchanger 1 & Slc9a1 & S707 \\
\hline STE20-like serine/threonine-protein kinase & Slk & S645 \\
\hline STE20-like serine/threonine-protein kinase & Slk & S649 \\
\hline Serine/threonine-protein kinase 24 & Stk24 & S4 \\
\hline Stathmin & Stmn1 & S16 \\
\hline Stathmin & Stmn1 & S16 \\
\hline Stathmin & Stmn1 & S25 \\
\hline Syntaxin-binding protein 1 & Stxbp1 & S507 \\
\hline Syntaxin-binding protein 1 & Stxbp1 & S509 \\
\hline Synapsin-1 & Syn1 & S67 \\
\hline Synapsin-1 & Syn1 & S664 \\
\hline Synapsin-2 & Syn2 & S524 \\
\hline Nesprin-1 & Syne1 & S2320 \\
\hline Transgelin-3 & Tagln3 & S163 \\
\hline Serotransferrin & $\mathrm{Tf}$ & $\mathrm{T} 212$ \\
\hline Thymosin beta- 4 & Tmsb4x & $\mathrm{T} 23$ \\
\hline Tubulin polymerization-promoting protein & Tppp & S159 \\
\hline Tubulin polymerization-promoting protein & Tppp & S34 \\
\hline E3 ubiquitin-protein ligase TRIM32 & Trim32 & S330 \\
\hline Ubiquitin-like modifier-activating enzyme 1 & Uba1 & S810 \\
\hline WD repeat-containing protein 44 & Wdr44 & S24 \\
\hline
\end{tabular}

Table 6-6: List of downregulated phosphosites upon CaMKII inhibition.

\begin{tabular}{|l|l|l|}
\hline Protein names & Gene names & $\begin{array}{l}\text { Aminoacid/ } \\
\text { Position }\end{array}$ \\
\hline Alpha-adducin & Add1 & S355 \\
\hline Alpha-adducin & Add1 & S600 \\
\hline Alpha-adducin & Add1 & T610 \\
\hline A-kinase anchor protein 12 & Akap12 & S364 \\
\hline A-kinase anchor protein 12 & Akap12 & S509 \\
\hline AMP deaminase 2 & Ampd2 & S21 \\
\hline Ankyrin-2 & Ank2 & T2238 \\
\hline Ankyrin repeat domain-containing protein 34A & Ankrd34a & S472 \\
\hline Adaptor-related protein complex 3, delta 1 subunit & Ap3d1 & S825 \\
\hline AP-3 complex subunit delta-1 & Ap3d1 & S755 \\
\hline AP-3 complex subunit delta-1 & Ap3d1 & S760 \\
\hline
\end{tabular}




\begin{tabular}{|c|c|c|}
\hline AP-3 complex subunit delta-1 & Ap3d1 & T758 \\
\hline Rho GTPase-activating protein 1 & Arhgap1 & S27 \\
\hline Rho GTPase-activating protein 44 & Arhgap44 & S799 \\
\hline Armadillo repeat-containing protein 10 & Armc10 & S43 \\
\hline Sodium/potassium-transporting ATPase subunit alpha-1 & Atp1a1 & S228 \\
\hline Sodium/potassium-transporting ATPase subunit alpha-3 & Atp1a3 & S442 \\
\hline Brain acid soluble protein 1 & Basp1 & S54 \\
\hline Serine/threonine-protein kinase BRSK2 & Brsk2 & S387 \\
\hline Protein bassoon & Bsn & S3497 \\
\hline Protein bassoon & Bsn & S1220 \\
\hline Protein bassoon & Bsn & S2844 \\
\hline Protein bassoon & Bsn & S2850 \\
\hline C2 domain-containing protein 2-like & C2cd2l & S59 \\
\hline C2 domain-containing protein 2-like & C2cd2l & T417 \\
\hline Voltage-dependent N-type calcium channel subunit alpha-1B & Cacna1b & S2023 \\
\hline Calcium/calmodulin-dependent protein kinase type II subunit alpha & Camk2a & S279 \\
\hline Calcium/calmodulin-dependent protein kinase type II subunit beta & Camk2b & S395 \\
\hline Calcium/calmodulin-dependent protein kinase type II subunit beta & Camk2b & T287 \\
\hline Calcium/calmodulin-dependent protein kinase type II subunit beta; & Camk2b & S276 \\
\hline Calcium/calmodulin-dependent protein kinase type II subunit beta & Camk2b & S280 \\
\hline Caskin-1 & Caskin1 & S1258 \\
\hline Caskin-1 & Caskin1 & S936 \\
\hline Coiled-coil domain-containing protein $85 \mathrm{~A}$ & Ccdc85a & S342 \\
\hline Protein Ccdc92 & Ccdc92 & S194 \\
\hline Cell cycle exit and neuronal differentiation protein 1 & Cend1 & S90 \\
\hline Cofilin-1 & Cfl1 & S3 \\
\hline Cofilin-2 & Cfl2 & S3 \\
\hline Claudin-11 & Cldn11 & S194 \\
\hline Claudin-11 & Cldn11 & S198 \\
\hline Claudin-11 & Cldn11 & S194 \\
\hline Claudin-11 & Cldn11 & S197 \\
\hline Claudin-11 & Cldn11 & S198 \\
\hline C-type lectin domain family 2 member $\mathrm{L}$ & Clec2l & S47 \\
\hline C-type lectin domain family 2 member $\mathrm{L}$ & Clec2l & S52 \\
\hline Cannabinoid receptor 1 & Cnr1 & S317 \\
\hline Complexin-1 & Cplx1 & S93 \\
\hline Complexin-2 & Cplx2 & S93 \\
\hline Dihydropyrimidinase-related protein 1 & Crmp1 & S542 \\
\hline Cytoplasmic FMR1-interacting protein 1 & Cyfip1 & T1234 \\
\hline Sn1-specific diacylglycerol lipase alpha & Dagla & S808 \\
\hline D-aspartate oxidase & Ddo & S197 \\
\hline Disks large homolog 2 & Dlg2 & S365 \\
\hline
\end{tabular}




\begin{tabular}{|c|c|c|}
\hline DmX-like protein 2 & Dmxl2 & S477 \\
\hline Dihydropyrimidinase-related protein 2 & Dpysl2 & S542 \\
\hline Dihydropyrimidinase-related protein 2 & Dpysl2 & T509 \\
\hline Dihydropyrimidinase-related protein 3 & Dpysl3 & T509 \\
\hline Cytoplasmic dynein 1 light intermediate chain 1 & Dync1li1 & S207 \\
\hline Band 4.1-like protein 3 & Epb4113 & S446 \\
\hline Band 4.1-like protein 3 & Epb4113 & $\mathrm{T} 472$ \\
\hline Dematin & Epb49 & S226 \\
\hline Protein Eps15l1 & Eps15l1 & S229 \\
\hline Protein Fam171b & Fam171b & S790 \\
\hline Gamma-aminobutyric acid type B receptor subunit 2 & Gabbr2 & S883 \\
\hline Gamma-aminobutyric acid type B receptor subunit 2 & Gabbr2 & T818 \\
\hline Neuromodulin & Gap43 & S142 \\
\hline Glutamate dehydrogenase 1, mitochondrial & Glud1 & S79 \\
\hline Glutamate dehydrogenase 1 , mitochondrial & Glud1 & S450 \\
\hline Gephyrin;Molybdopterin adenylyltransferase & Gphn & S192 \\
\hline Neuronal membrane glycoprotein M6-a & Gpm6a & S256 \\
\hline Neuronal membrane glycoprotein M6-a & Gpm6a & S270 \\
\hline Probable G-protein coupled receptor 158 & Gpr158 & S902 \\
\hline Probable G-protein coupled receptor 158 & Gpr158 & S904 \\
\hline Glutamate receptor ionotropic, delta-2 & Grid2 & S816 \\
\hline Glutamate receptor ionotropic, delta-2 & Grid2 & S809 \\
\hline Glutamate receptor ionotropic, delta-2 & Grid2 & S816 \\
\hline Glutamate receptor ionotropic, NMDA 2B & Grin2b & S1303 \\
\hline Heat shock $70 \mathrm{kDa}$ protein $4 \mathrm{~L}$ & Hspa4l & S74 \\
\hline Protein IMPACT & Impact & S295 \\
\hline Inositol polyphosphate 5-phosphatase OCRL-1 & Inpp5f & S123 \\
\hline Intersectin-1 & Itsn1 & S975 \\
\hline Potassium voltage-gated channel subfamily A member 2 & Kcna2 & S434 \\
\hline Kinesin-like protein KIF21A & Kif21a & S1227 \\
\hline Kinesin light chain 3 & Klc2 & S151 \\
\hline Plastin-2 & Lcp1 & S5 \\
\hline Ubiquitin carboxyl-terminal hydrolase & LOC100911959 & S783 \\
\hline Ephexin-1 & LOC100912512 & S512 \\
\hline Protein LOC501194 & LOC501194 & S38 \\
\hline Protein LOC681198 & LOC681198 & Y52 \\
\hline PRotein LOC688905 & LOC688905 & S249 \\
\hline Leucine-rich repeat-containing protein 7 & Lrrc7 & S949 \\
\hline Microtubule-actin cross-linking factor 1 & Macf1 & S2454 \\
\hline MAP kinase-activating death domain protein & Madd & S1038 \\
\hline MAP kinase-activating death domain protein & Madd & T1045 \\
\hline $\begin{array}{l}\text { Membrane-associated guanylate kinase, WW and PDZ domain-containing protein } \\
1\end{array}$ & Magi1 & S528 \\
\hline
\end{tabular}




\begin{tabular}{|c|c|c|}
\hline Microtubule-associated protein $1 \mathrm{~A}$ & Map1a & S907 \\
\hline Microtubule-associated protein 1B & Map1b & S1305 \\
\hline Microtubule-associated protein 1B & Map1b & S609 \\
\hline Microtubule-associated protein 1B & Map1b & S614 \\
\hline Microtubule-associated protein 1B & Map1b & S1382 \\
\hline Microtubule-associated protein 4 & Map4 & S522 \\
\hline Microtubule-associated protein 6 & Map6 & S681 \\
\hline Microtubule-associated protein tau;Microtubule-associated protein & Mapt & S253 \\
\hline Microtubule-associated protein tau;Microtubule-associated protein & Mapt & T208 \\
\hline Microtubule-associated protein tau;Microtubule-associated protein & Mapt & S391 \\
\hline Serine/threonine-protein kinase MARK1 & Mark1 & T198 \\
\hline Myelin basic protein & Mbp & S139 \\
\hline Myelin basic protein & Mbp & T122 \\
\hline Malate dehydrogenase, cytoplasmic & Mdh1 & S241 \\
\hline Protein-methionine sulfoxide oxidase MICAL3 & Mical3 & S976 \\
\hline Misshapen-like kinase 1 & Mink1 & S777 \\
\hline Neurobeachin & Nbea & S372 \\
\hline Neural cell adhesion molecule 1 & Ncam1 & S760 \\
\hline Neural cell adhesion molecule 1 & Ncam1 & S764 \\
\hline NCK-interacting protein with SH3 domain & Nckipsd & S122 \\
\hline Nuclear distribution protein nudE-like 1 & Ndel1 & S198 \\
\hline Neurofilament heavy polypeptide & Nefh & S872 \\
\hline Neurofilament heavy polypeptide & Nefh & S838 \\
\hline Neurofilament heavy polypeptide & Nefh & S774 \\
\hline Neurofilament heavy polypeptide & Nefh & S538 \\
\hline Neurofilament heavy polypeptide & Nefh & S670 \\
\hline Neurofilament heavy polypeptide & Nefh & S580 \\
\hline Neurofilament heavy polypeptide & Nefh & S658 \\
\hline Neurofilament heavy polypeptide & Nefh & S646 \\
\hline Neurofilament heavy polypeptide & Nefh & S61 \\
\hline Neurofilament medium polypeptide & Nefm & S713 \\
\hline Neurofilament medium polypeptide & Nefm & S721 \\
\hline Neurofilament medium polypeptide & Nefm & S713 \\
\hline Neurofilament medium polypeptide & Nefm & S721 \\
\hline Neurofibromin & Nf1 & S2512 \\
\hline Oxidation resistance protein 1 & Oxr1 & S470 \\
\hline Oxidation resistance protein 1 & Oxr1 & S179 \\
\hline Paralemmin-1 & Palm & T145 \\
\hline Paralemmin-1 & Palm & S124 \\
\hline Protein piccolo & Pclo & S3565 \\
\hline Protein piccolo & Pclo & S1575 \\
\hline Protein piccolo & Pclo & S3626 \\
\hline
\end{tabular}




\begin{tabular}{|c|c|c|}
\hline Protein piccolo & Pclo & S3632 \\
\hline cAMP-specific 3',5'-cyclic phosphodiesterase 4A & Pde4a & S15 \\
\hline Pyruvate dehydrogenase E1 component subunit alpha, somatic form & Pdha1 & S232 \\
\hline Pyruvate dehydrogenase E1 component subunit alpha, somatic form & Pdha1 & S300 \\
\hline Pyruvate dehydrogenase E1 component subunit alpha, somatic form & Pdha1 & S295 \\
\hline PEX5-related protein & Pex5l & S232 \\
\hline Phosphatidylinositol 4-kinase type 2-alpha & Pi4k2a & S46 \\
\hline Plectin & Plec & S580 \\
\hline Plexin domain-containing protein 2 & Plxdc2 & S507 \\
\hline Liprin-alpha-3 & Ppfia3 & S1162 \\
\hline Protein phosphatase $1 \mathrm{H}$ & Ppm1h & S220 \\
\hline Protein phosphatase 1 regulatory subunit 1B & Ppp1r1b & S102 \\
\hline Serine/threonine-protein phosphatase 2B catalytic subunit beta isoform & Ppp3cb & S481 \\
\hline cAMP-dependent protein kinase catalytic subunit alpha & Prkaca; & T190 \\
\hline cAMP-dependent protein kinase type II-alpha regulatory subunit & Prkar2a & S97 \\
\hline Protein kinase C;Protein kinase $\mathrm{C}$ alpha type & Prkca & T500 \\
\hline Protein kinase $\mathrm{C}$ beta type & Prkcb & T642 \\
\hline Protein kinase $\mathrm{C}$ epsilon type & Prkce & S349 \\
\hline Protein kinase $\mathrm{C}$ epsilon type & Prkce & S345 \\
\hline Protein kinase $\mathrm{C}$ epsilon type & Prkce & S349 \\
\hline Protein Prrt3 & Prrt3 & S807 \\
\hline Protein-tyrosine kinase 2-beta & Ptk2b & S375 \\
\hline Very-long-chain (3R)-3-hydroxyacyl-CoA dehydratase 3 & Ptplad1 & S114 \\
\hline Poliovirus receptor-related 1 & Pvrl1 & S421 \\
\hline Protein RGD1306271 & RGD1306271 & S30 \\
\hline Protein RGD1306271 & RGD1306271 & $\mathrm{T} 28$ \\
\hline Protein RGD1307235 & RGD1307235 & S244 \\
\hline Protein RGD1310819 & RGD1310819 & S479 \\
\hline Protein RGD1559864 & RGD1559864 & $\mathrm{T} 47$ \\
\hline Regulating synaptic membrane exocytosis protein 1 & Rims1 & S1538 \\
\hline Regulating synaptic membrane exocytosis protein 1 & Rims1 & S1277 \\
\hline Regulating synaptic membrane exocytosis protein 1 & Rims1 & S413 \\
\hline Regulating synaptic membrane exocytosis protein 1 & Rims1 & S991 \\
\hline Regulating synaptic membrane exocytosis protein 2 & Rims2 & S177 \\
\hline Rabphilin-3A & Rph3a & S683 \\
\hline Ras-related protein R-Ras2 & Rras2 & S186 \\
\hline Septin-5 & Sep5 & S234 \\
\hline Septin-5 & Sep5 & T13 \\
\hline Serine incorporator 1 & Serinc1 & S364 \\
\hline SH3-containing GRB2-like protein 3-interacting protein 1 & Sgip1 & S293 \\
\hline SH3-containing GRB2-like protein 3-interacting protein 1 & Sgip1 & S169 \\
\hline SH3-containing GRB2-like protein 3-interacting protein 1 & Sgip1 & S170 \\
\hline
\end{tabular}




\begin{tabular}{|c|c|c|}
\hline SH3-containing GRB2-like protein 3-interacting protein 1 & Sgip1 & S319 \\
\hline SH3 and multiple ankyrin repeat domains protein 1 & Shank1 & S1138 \\
\hline SH3 and multiple ankyrin repeat domains protein 2 & Shank2 & S1031 \\
\hline Monocarboxylate transporter 1 & Slc16a1 & S210 \\
\hline Monocarboxylate transporter 1 & Slc16a1 & S213 \\
\hline Excitatory amino acid transporter 1 & Slc1a3 & S466 \\
\hline ADP/ATP translocase 1 & Slc25a4 & Y195 \\
\hline Vesicular inhibitory amino acid transporter & Slc32a1 & S97 \\
\hline Anion exchange protein 3 & Slc4a3 & S296 \\
\hline Sodium/hydrogen exchanger 1 & Slc9a1 & S697 \\
\hline Protein Sntb2 & Sntb2 & S77 \\
\hline SRC kinase signaling inhibitor 1 & Srcin 1 & S941 \\
\hline SRC kinase signaling inhibitor 1 & Srcin 1 & S556 \\
\hline SRC kinase signaling inhibitor 1 & Srcin 1 & S1077 \\
\hline Stathmin & Stmn1 & S16 \\
\hline Stathmin & Stmn1 & S16 \\
\hline Stathmin & Stmn1 & S25 \\
\hline Stathmin;Stathmin-2 & Stmn1;Stmn2 & S46 \\
\hline Syntaxin-binding protein 5-like & Stxbp5l & S574 \\
\hline Syntaxin-binding protein 5-like & Stxbp5l & S797 \\
\hline Syntaxin-binding protein 5-like & Stxbp5l & S798 \\
\hline Synapsin-1 & Syn1 & S566 \\
\hline Synapsin-2 & Syn2 & S524 \\
\hline Synapsin-2;Synapsin-1 & Syn2;Syn1 & S697 \\
\hline Synapsin-3 & Syn3 & S572 \\
\hline Synapsin-3 & Syn3 & S540 \\
\hline Synaptophysin & Syp & $\mathrm{Y} 75$ \\
\hline Synaptotagmin-17 & Syt17 & S110 \\
\hline Synaptotagmin-2 & Syt2 & T128 \\
\hline Synaptotagmin-7 & Syt7 & T58 \\
\hline Transgelin-3 & Tagln3 & S163 \\
\hline Serotransferrin & Tf & $\mathrm{T} 492$ \\
\hline Tumor protein D53 & Tpd52l1 & S137 \\
\hline Tumor protein D54 & Tpd52l2 & S168 \\
\hline Tubulin polymerization-promoting protein & Tppp & T15 \\
\hline Tubulin polymerization-promoting protein & Tppp & S31 \\
\hline Tubulin polymerization-promoting protein & Tppp & S34 \\
\hline Trafficking protein particle complex subunit 9 & Trappc9 & S766 \\
\hline Tripartite motif-containing protein 2 & Trim2 & S424 \\
\hline Tripartite motif-containing protein 2 & Trim2 & S428 \\
\hline (E3-independent) E2 ubiquitin-conjugating enzyme & Ube2o & S892 \\
\hline (E3-independent) E2 ubiquitin-conjugating enzyme & Ube2o & S81 \\
\hline
\end{tabular}




\begin{tabular}{|l|l|l|}
\hline (E3-independent) E2 ubiquitin-conjugating enzyme & Ube2o & S83 \\
\hline Ubiquitin fusion degradation protein 1 homolog & Ufd1l & S299 \\
\hline Protein Wdfy3 & Wdfy3 & S1942 \\
\hline WD repeat-containing protein 37 & Wdr37 & S22 \\
\hline
\end{tabular}




\subsection{Acknowledgements}

I would like to thank Prof. Dr. Reinhard Jahn for his constant support during this project. Reinhard, thank you for sharing with us your enthusiasm for science and brilliant ideas, every time after meeting with you I was reminded that the world of science is so wonderful and interesting and I am grateful for this. It was an honor for me to work in your group.

I thank Prof. Henning Urlaub for his support and scientific input in the Mass Spectrometry part of this project. Henning, thank you for letting me integrate to your group and using your precious, expensive instruments.

I also would like to thank Prof. Jürgen Wienands for being part of my thesis committee and for the discussions and inputs during the thesis committee meetings.

I thank my collaborators in the Neurobiology Department: Dr. Dominika Czernik, Dr. Ángel PérezLara and Dr. John Chua for their scientific inputs and technical assistance. In addition, I would like to thank all the former and present member of the department especially Seichii, Shruttee, Dragomir, Hale, Agata, Marcello, Vedran, Claudia, and Vindya. Special thanks go to Tahere for proofreading my thesis, my student, Deniz Kaya, working with whom was a pleasure, Dr. Janina Boyken for teaching me the synaptosome preparation and Łukasz Jabłoński for helping me to set up the electrical stimulation method.

I would like to thank Dr. Gotfried Meiskes, Dr. Hans Dieter Schmitt and Elisa Reckmann-Heinrich for their help with lab-related issues. I would also express an additional thank to Dieter for sharing his office with me during the writing phase. I thank Brigitte Barg-Kues, Sigrid Schmidt, and Dagmar Diezmann for their technical assistance.

I would like to thank all the members of Bioanalytical Mass Spectrometry Group especially, Uwe Pleßmann, Monika Raabe, and Annika Kühn for their excellent technical support in maintaining of the Mass Spectrometry Instruments. I also thank, Dr. Kuan-Ting Pan for very helpful discussions and critical review of my paper and thesis. Thank you Kuan-Ting, I learned a lot from you. I also thank Dr. Jasmin Corso and Dr. Sunit Mandad for their helpful discussions and technical assistance. I thank members of animal facility of MPI-bpc especially Sascha Krause and Thomas Gundlach for their help with animal delivery and taking care of rats.

I want to thank my PhD program, International Max Planck Research School for Molecular Biology, for financial support during my thesis and also for providing great opportunities to grow and develop through the courses, organization of events and interesting excursions. Particularly, I 
would like to express my gratitude to Dr. Steffen Burkhardt and Kerstin Grüniger for their excellent help, and council from the first day of my arrival in Germany.

I would like to thank specially Dr. Zohreh Farsi not only for being an excellent colleague but also a very supportive friend.

My great thank goes to Sasha, who was always there for me with a smile to calm me down from all my frustrations and worries, reminding me everything is and will be great. Sashko, life with you is much more beautiful.

Finally, I would like to thank my family, who is my strength in life. I am a lucky person to have a great family that persuaded me to follow my dreams, support me in any way they can and love me unconditionally. مرسى مامان، بابا، مريم و كاوه عزيز 